\title{
Diastereo- and Enantioselective Construction of Spirocycles by Nickel-Catalyzed Cascade Borrowing Hydrogen Cyclization
}

\author{
Zhengtian Ding, Yiming Wang, Wenfeng Liu, Yate Chen, and Wangqing Kong* \\ The Center for Precision Synthesis (CPS), Institute for Advanced Studies (IAS), Wuhan \\ University, 299 Bayi Road, Wuchang District, Wuhan, Hubei 430072, P. R. China
}

email: wqkong@whu.edu.cn 


\section{Table of Contents}

1. General Information S3

2. General procedure S4

3. Mechanistic experiments S5

4. Characterization data of products $\quad \mathrm{S} 18$

5. Gram-scale reactions and synthetic transformations $\quad \mathrm{S} 92$

6. Single crystal X-ray diffraction of $\mathbf{3 a}$ and $\mathbf{3 u} \quad \mathbf{S} 99$

7. Copies of the ${ }^{1} \mathrm{H},{ }^{19} \mathrm{~F}$ NMR and ${ }^{13} \mathrm{C}$ NMR spectra $\quad \mathrm{S} 122$

$\begin{array}{lll}\text { 8. References } & \text { S173 }\end{array}$ 


\section{General information}

${ }^{1} \mathrm{H}$ and ${ }^{13} \mathrm{C}$ NMR data were recorded with Bruker ADVANCE III (400 MHz) or JNM-ECZ400S/L1 (400 MHz) spectrometers. Chemical shifts are given in ppm. The spectra are calibrated to the residual ${ }^{1} \mathrm{H}$ and ${ }^{13} \mathrm{C}$ signals of the solvents. Multiplicities are abbreviated as follows: singlet (s), doublet (d), triplet (t), quartet (q), doublet-doublet (dd), quintet (quint), septet (sept), multiplet (m), and broad (b). ${ }^{19} \mathrm{~F} \mathrm{NMR}$ spectra were recorded using $\mathrm{CFCl}_{3}$ as internal standard. Gas chromatography were determined with a SHIMADZU Nexis GC 2030 gas chromatography instrument with a FID detector. High-resolution mass spectra (HRMS) were recorded on Thermo Fisher Orbitrap Elite mass spectrometer. Enantiomeric excesses were determined with a SHIMADZU LC-20ADXR system using chiral stationary phase columns (DAICEL) by comparing the samples with the corresponding racemic samples. Column and elution details were specified in each entry.

Unless otherwise stated, starting materials were purchased from commercial suppliers (Energy Chemical, Alfa, Aldrich and so on). All reactions dealing with air- or moisture-sensitive compounds were performed in the argon-filled glove box or by standard Schlenk techniques in oven-dried reaction vessels under argon atmosphere. Solvents were purchased in HPLC quality, degassed by purging thoroughly with argon and dried over activated molecular sieves of appropriate size. More sensitive compounds were stored in a desiccator or in a glove-box if required. Reactions were monitored by thin layer chromatography (TLC) using glass $0.25 \mathrm{~mm}$ silica gel plates. Compounds were visualized by UV-light at $254 \mathrm{~nm}$ and by dipping the plates in an aqueous potassium permanganate solution followed by heating. Flash column chromatography was performed over silica gel (200-400 mesh). 


\section{General procedure}

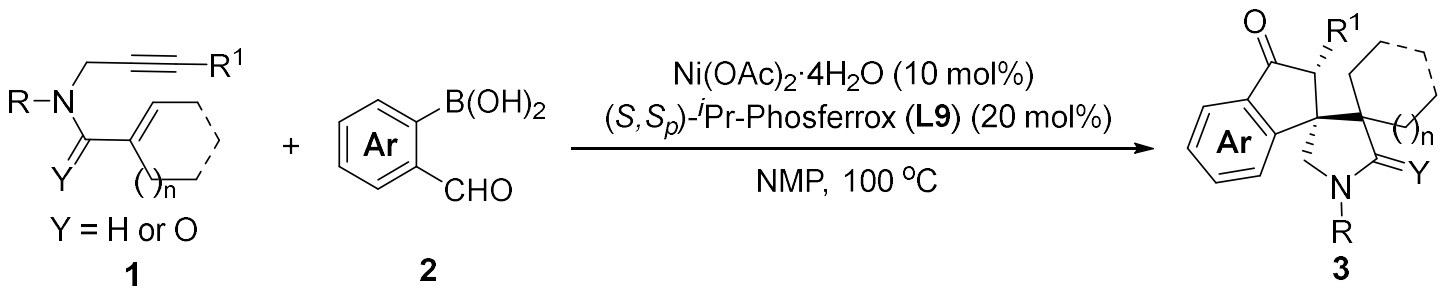

An oven-dried sealed tube equipped with a PTFE-coated stir bar was charged with $\mathrm{Ni}(\mathrm{OAc})_{2} \cdot 4 \mathrm{H}_{2} \mathrm{O}$ (10 mol\%), (S, Sp)-iPr-Phosferrox (L9) (20 mol\%) and o-formylarylboronic acid 2 (3.0 equiv). Solid substrate 1 (1.0 equiv) was also added at this stage. The sealed tube was evacuated and backfilled with argon (this process was repeated for three times) and then NMP $(0.05 \mathrm{M})$ or a solution of the liquid substrate 1 (1.0 equiv in NMP $(0.05 \mathrm{M}))$ was added. This reaction mixture was stirred at room temperature for 15 minutes and then heated at $100^{\circ} \mathrm{C}$ for 36 hours until the reaction was complete (monitored by TLC). The resulting mixture was quenched with sat. $\mathrm{NH}_{4} \mathrm{Cl}$ solution $(5 \mathrm{~mL})$ and further diluted with water $(10 \mathrm{~mL})$. The aqueous layer was extracted with EtOAc $(3 \times 15 \mathrm{~mL})$ and the combined organic layers were washed with brine $(2 \times 20 \mathrm{~mL})$, dried with $\mathrm{MgSO}_{4}$, filtered, and concentrated under reduced pressure. The residue was purified by chromatography on silica gel, eluting with ethyl acetate/petroleum ether 1:20 1:5 (v/v) to afford the corresponding product 3 . 


\section{Mechanistic experiments}

\section{Proposed mechanism 1:1}

Oxidative cyclization of enyne 1 with $\mathrm{Ni}(0)$ affords the nickelacycle intermediate I. Migratory insertion of aldehyde $\mathbf{2}$ into the nickelacycle species I gives seven-membered alkoxynickel intermediate II, which undergoes $\beta$-H elimination and reductive elimination to form intermediate III and regenerate active $\mathrm{Ni}(0)$ species. Transmetalation of arylboronic acid with the nickel(II) catalyst gives arylnickel species IV. Migratory insertion of double bond into arylnickel species forms the $\mathrm{Csp}^{3}$-nickel intermediate $\mathbf{V}$, which can undergo hydrolysis to release the desired spiroindanone product 3 and regenerated the active nickel(II) catalyst.

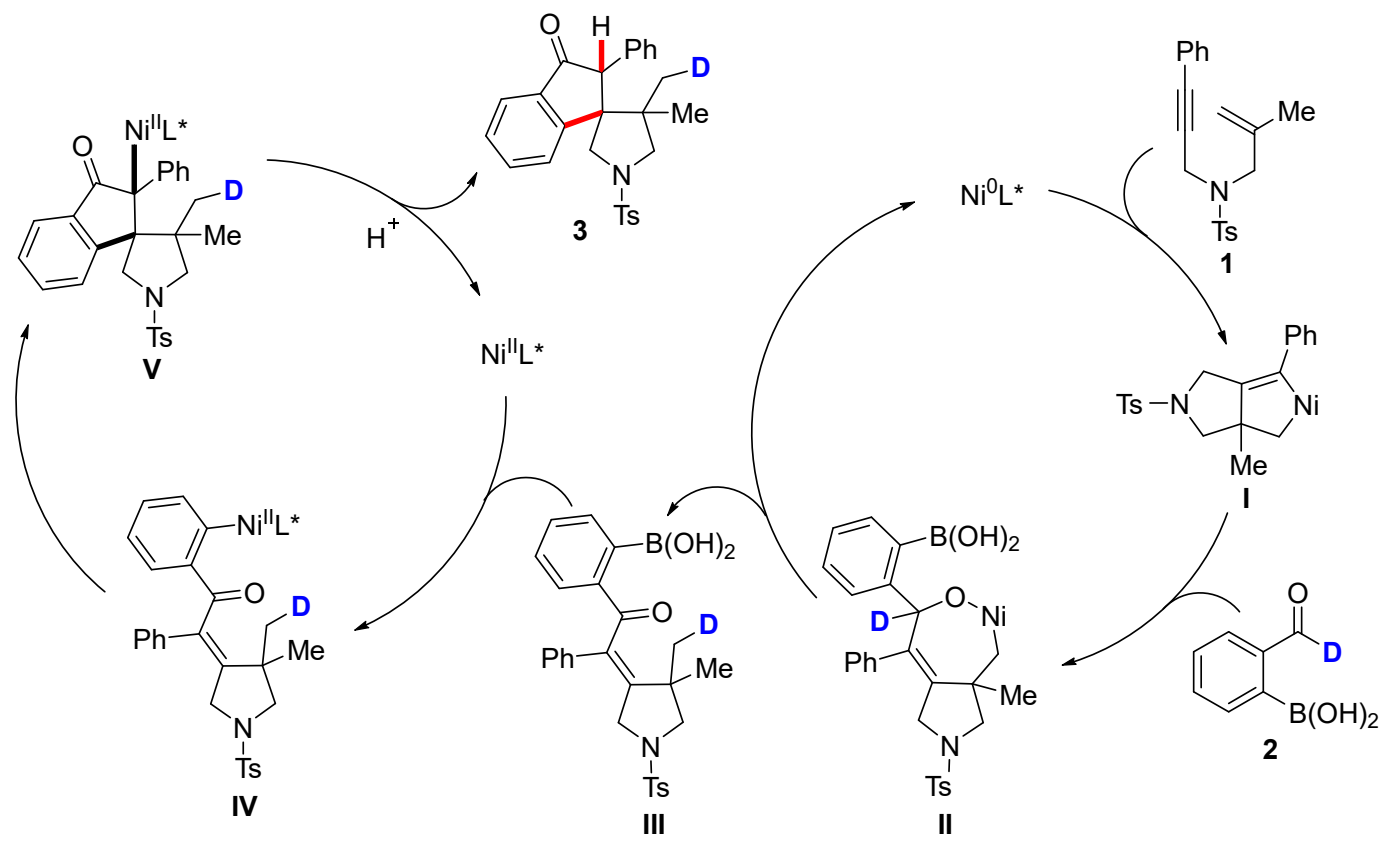

We performed the reactions of enyne $1 \mathrm{a}$ and benzaldehyde with or without phenylboronic acid. However, failure to observe product III' analogous to III suggests that this mechanism is not operative. 

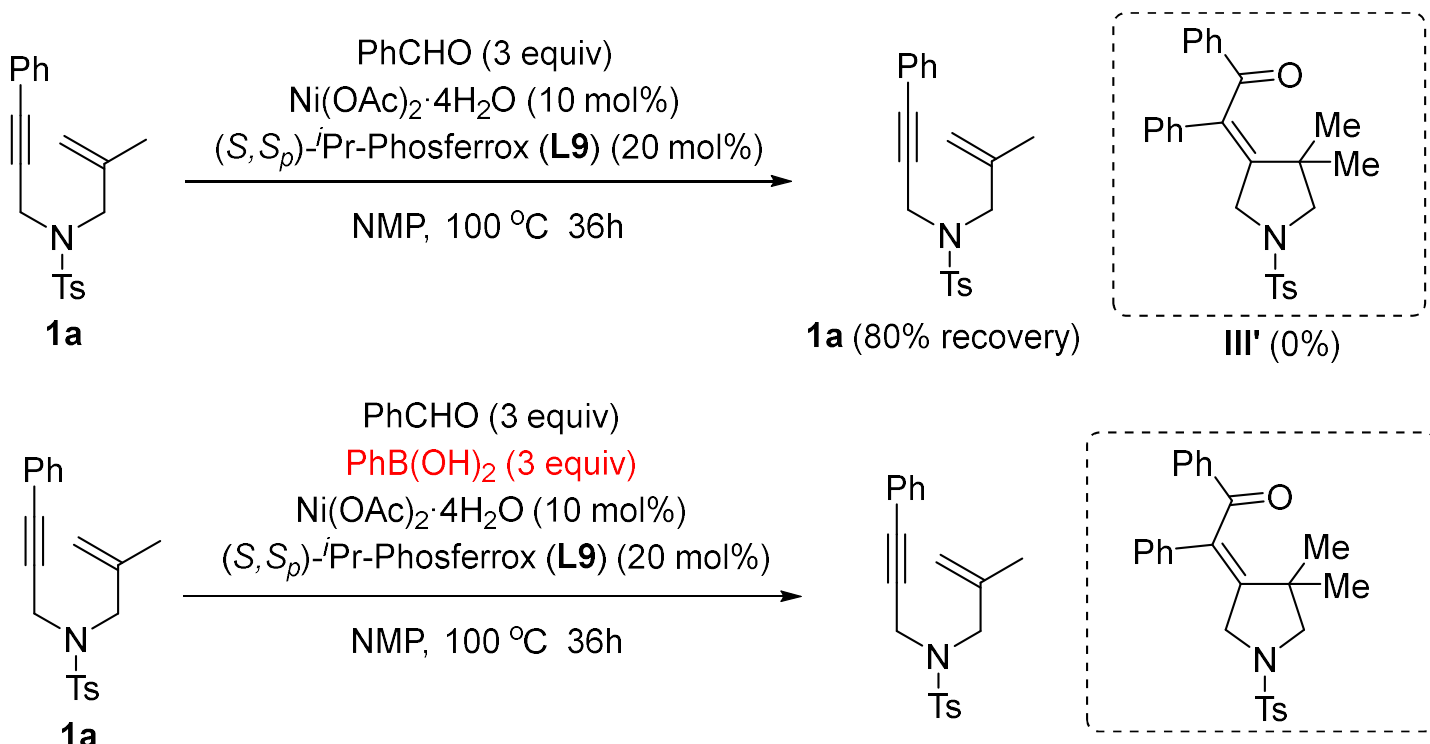

1a (92\% recovery)

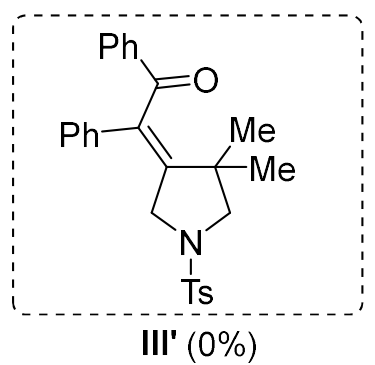

The kinetic profile of the reaction mixture of $1 \mathbf{a}$ and $\mathbf{2 a}$ under standard conditions shows that indenol $\mathbf{4 a}$ ' is rapidly formed and finally converted into the target product $\mathbf{3 a}$. However, the key alcohol intermediate $\mathbf{4}^{\prime}$ is not involved in the catalytic cycle, the reaction mechanism can be easily ruled out.<smiles>C=C(C)CN(C)CC#Cc1ccccc1</smiles>

$1 \mathrm{a}$<smiles>O=Cc1ccccc1Br</smiles>

$2 a$
$\mathrm{Ni}(\mathrm{OAc})_{2} \cdot 4 \mathrm{H}_{2} \mathrm{O}(10 \mathrm{~mol} \%)$ L9 (20 $\mathrm{mol} \%)$ NMP, $100^{\circ} \mathrm{C}$
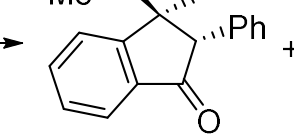

$3 a$

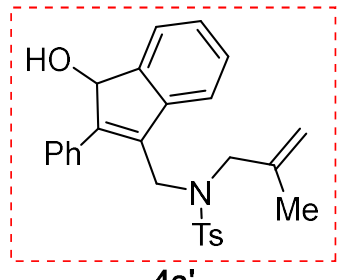

$4 a^{\prime}$

\begin{tabular}{lccc} 
reaction time & yield $(\%)$ of $3 a$ & yield $(\%)$ of $4 a^{\prime}$ \\
9 & 24 & 16 \\
\hline 36 hours & 76 & 0
\end{tabular}


Kinetic profile of the cascade reaction:

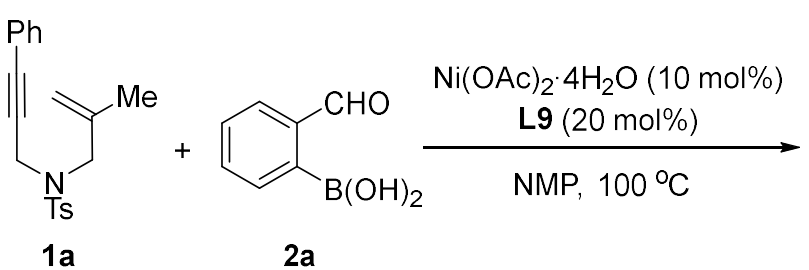

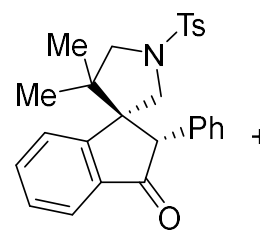

3a

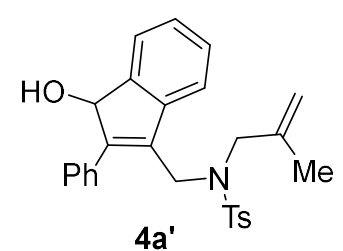

$4 a^{\prime}$

Five parallel standard reactions were carried out using substrate $\mathbf{1 a}$ and $\mathbf{2 a}$.

The reaction was quenched according to the set time, and the desired product $3 \mathbf{a}$ and alcohol intermediate $\mathbf{4 a}$ ' are isolated by silica gel column chromatography, and the corresponding starting material $1 \mathrm{a}$ was also recovered.

The reaction profile was collected, it was found that the alcohol intermediate $\mathbf{4 a}$ ' was formed rapidly and transformed into the desired product $\mathbf{3 a}$. 


\section{Deuterium atom transfer experiment:}

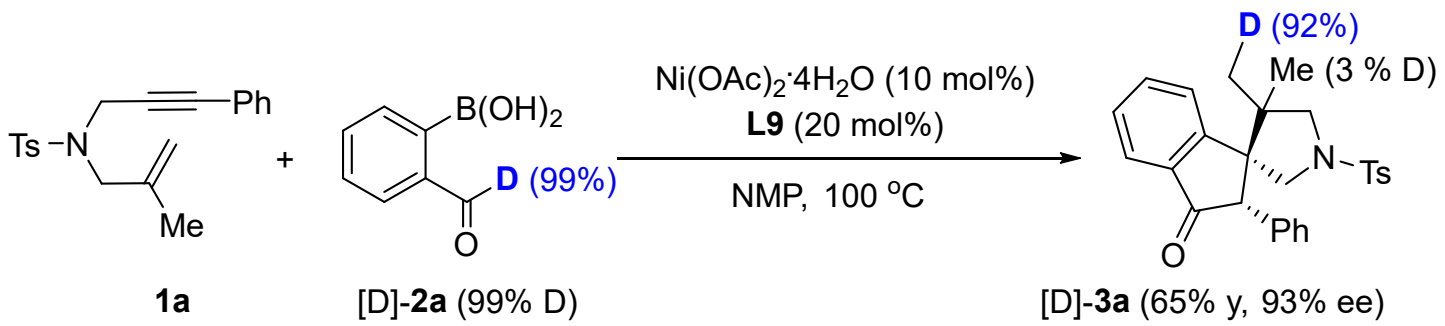

\section{General procedure for the deuterium atom transfer experiments:}

An oven-dried sealed tube equipped with a PTFE-coated stir bar was charged with $\mathrm{Ni}(\mathrm{OAc})_{2} \cdot 4 \mathrm{H}_{2} \mathrm{O}(0.01 \mathrm{mmol}, 2.5 \mathrm{mg}),(\mathrm{S}, \mathrm{Sp})^{-}{ }^{-} \mathrm{Pr}-\mathrm{Phosferrox}$ (L9) $(0.02 \mathrm{mmol}, 9.6 \mathrm{mg}),(2-$ formylphenyl)boronic acid [D]-2a $(0.3 \mathrm{mmol}, 45.3 \mathrm{mg})$ and $1 \mathrm{a}(0.1 \mathrm{mmol}, 33.9 \mathrm{mg})$. The sealed tube was evacuated and backfilled with argon (this process was repeated for three times) and then NMP $(2 \mathrm{~mL})$ was added. This reaction mixture was stirred at room temperature for 15 minutes and then heated at $100{ }^{\circ} \mathrm{C}$ for 36 hours until the reaction was complete. The resulting mixture was quenched with sat. $\mathrm{NH}_{4} \mathrm{Cl}$ solution $(5 \mathrm{~mL})$ and further diluted with water $(10 \mathrm{~mL})$. The aqueous layer was extracted with EtOAc $(3 \times 15 \mathrm{~mL})$ and the combined organic layers were washed with brine $(2 \times 20 \mathrm{~mL})$, dried with $\mathrm{MgSO}_{4}$, filtered, and concentrated under reduced pressure. The residue was purified by chromatography on silica gel, eluting with ethyl acetate/petroleum ether 1:10 1:5 (v/v) to afford the corresponding product [D]-3a (28.9 $\mathrm{mg}, 65 \%$ yield, $93 \%$ ee and $92 \% \mathrm{D}$ incorporation).
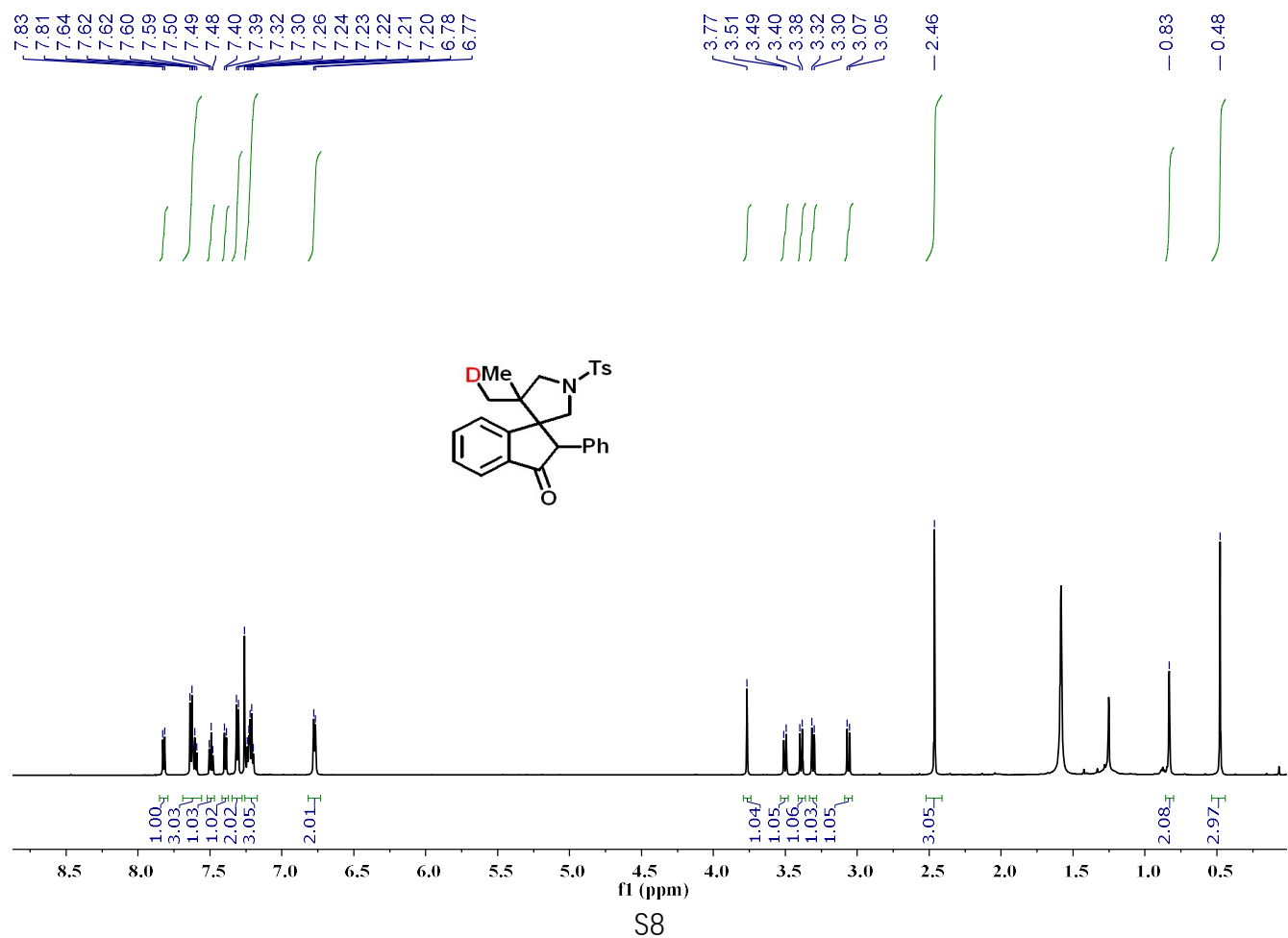


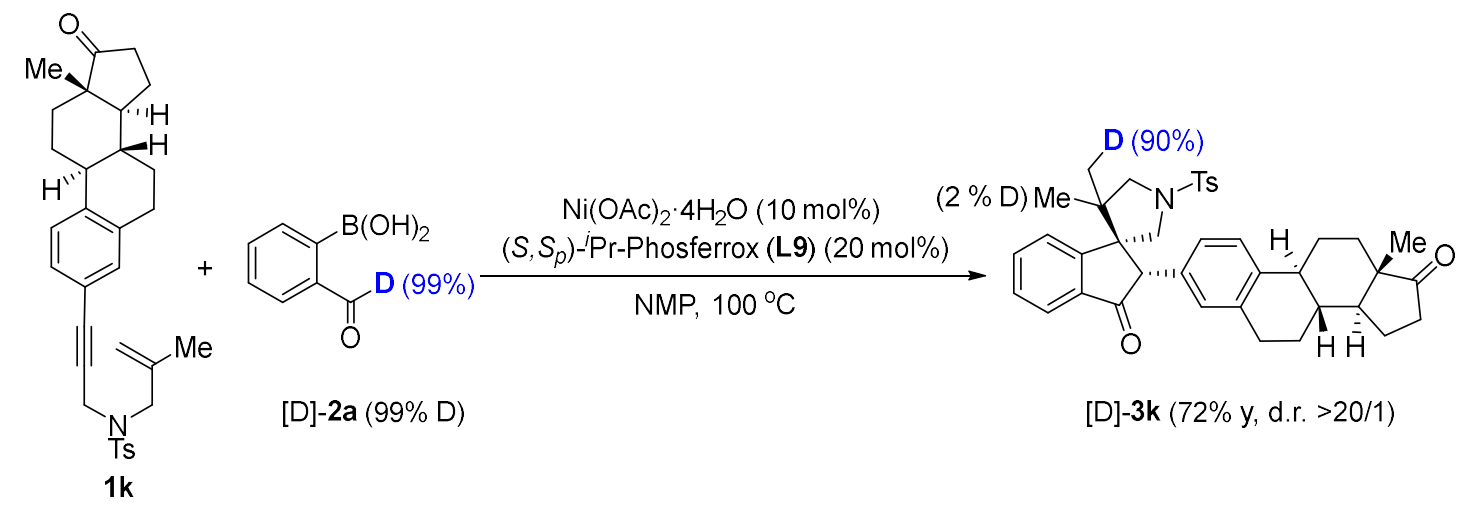

[D]-3k was prepared according to general procedure using $1 \mathbf{k}(0.1 \mathrm{mmol}, 51.5 \mathrm{mg})$ and $[\mathrm{D}]-2 \mathbf{a}$ ( $0.3 \mathrm{mmol}, 45.3 \mathrm{mg}$ ) and was purified by silica gel column chromatography (petroleum ether/ethyl acetate $=10 / 1 \sim 5 / 1)$ to obtain $[D]-3 k(44.6 \mathrm{mg}, 72 \%$ yield, $>20 / 1 \mathrm{~d} . \mathrm{r}$ and $90 \% \mathrm{D}$ incorporation).
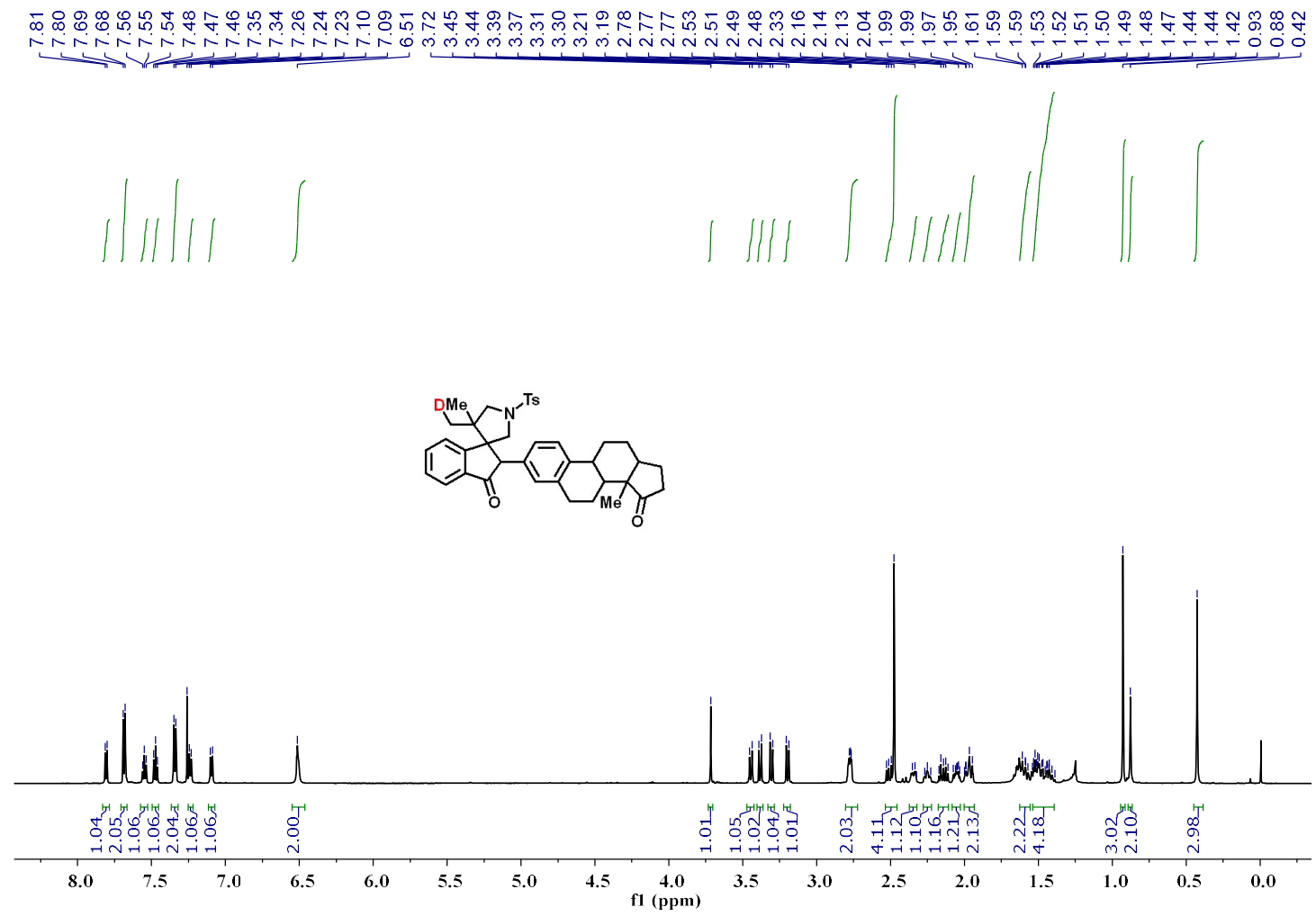


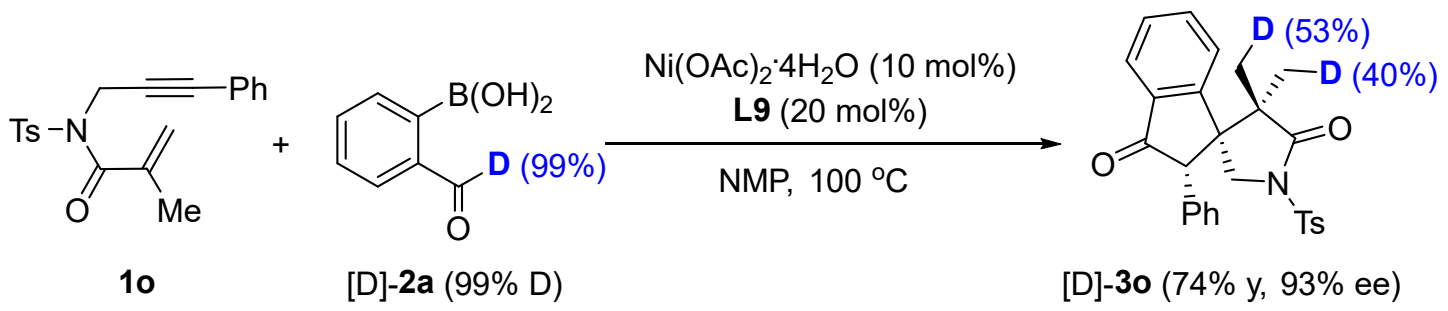

[D]-3o was prepared according to general procedure using $10(0.1 \mathrm{mmol}, 35.3 \mathrm{mg})$ and [D]-2a $(0.3 \mathrm{mmol}, 45.3 \mathrm{mg})$ and was purified by silica gel column chromatography (petroleum ether/ethyl acetate $=10 / 1 \sim 5 / 1)$ to obtain [D]-3o $(33.8 \mathrm{mg}, 74 \%$ yield, $93 \%$ ee and $93 \% \mathrm{D}$ incorporation)
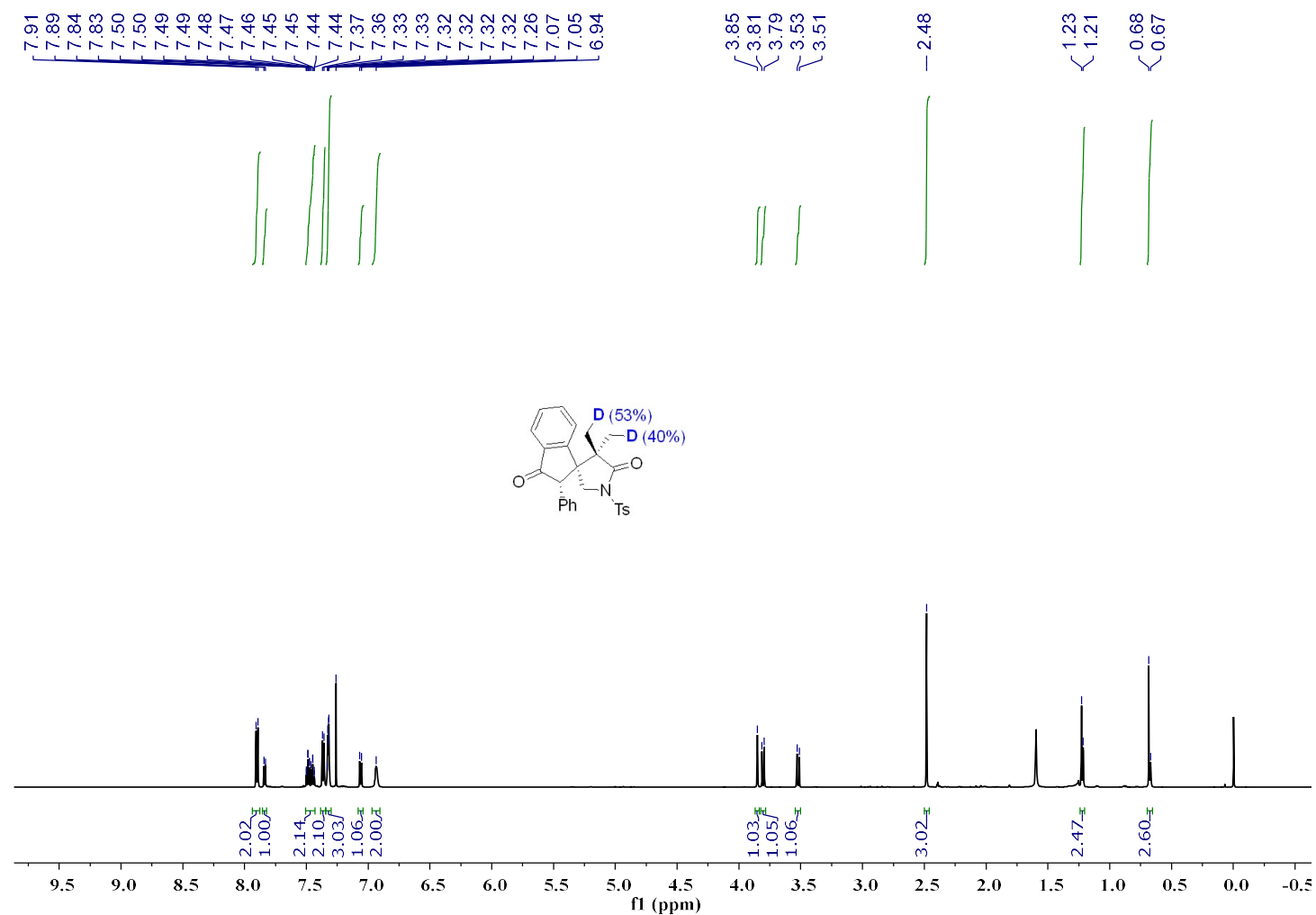

Interestingly, both methyl groups were deuterated in the product 3o. This result may be due to the $\beta-\mathrm{H}$ elimination after the migratory insertion of $\mathrm{Ni}-\mathrm{H}$ into the activated double bond, which further proved the existence of $\mathrm{Ni}-\mathrm{H}$ species. 
<smiles>C=CCN([Al])CC#Cc1ccccc1</smiles><smiles>O=C(O)c1ccccc1Br</smiles>

$[D]-2 a(99 \%$ D)

$1 \mathrm{p}$
$\mathrm{Ni}(\mathrm{OAc})_{2} \cdot 4 \mathrm{H}_{2} \mathrm{O}(10 \mathrm{~mol} \%)$ $\underset{\text { NMP, } 100{ }^{\circ} \mathrm{C}}{\stackrel{\left(S, S_{p}\right)-{ }^{i} \text { Pr-Phosferrox (L9) }(20 \mathrm{~mol} \%)}{\longrightarrow}}$ $\underset{\text { NMP, } 100^{\circ} \mathrm{C}}{\stackrel{\left.\left(S, S_{p}\right)\right)^{\prime} \text { Pr-Phosferrox }(\mathbf{L 9})(20 \mathrm{~mol} \%)}{\longrightarrow}}$

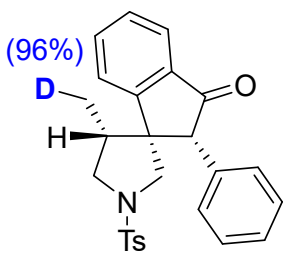

[D]-3p (76\% y, 90\% ee)

[D]-3p was prepared according to general procedure using $1 \mathbf{p}(0.1 \mathrm{mmol}, 32.5 \mathrm{mg})$ and $[\mathrm{D}]-2 \mathrm{a}$ (0.3 mmol, $45.3 \mathrm{mg}$ ) and was purified by silica gel column chromatography (petroleum ether/ethyl acetate $=10 / 1 \sim 5 / 1)$ to obtain $[D]-3 p(32.8 \mathrm{mg}, 76 \%$ yield, $90 \%$ ee and $96 \% \mathrm{D}$ incorporation).

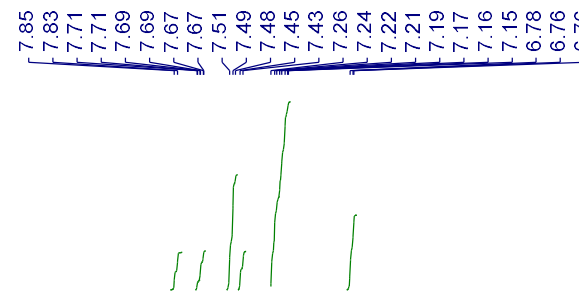
$\underbrace{2000000}$
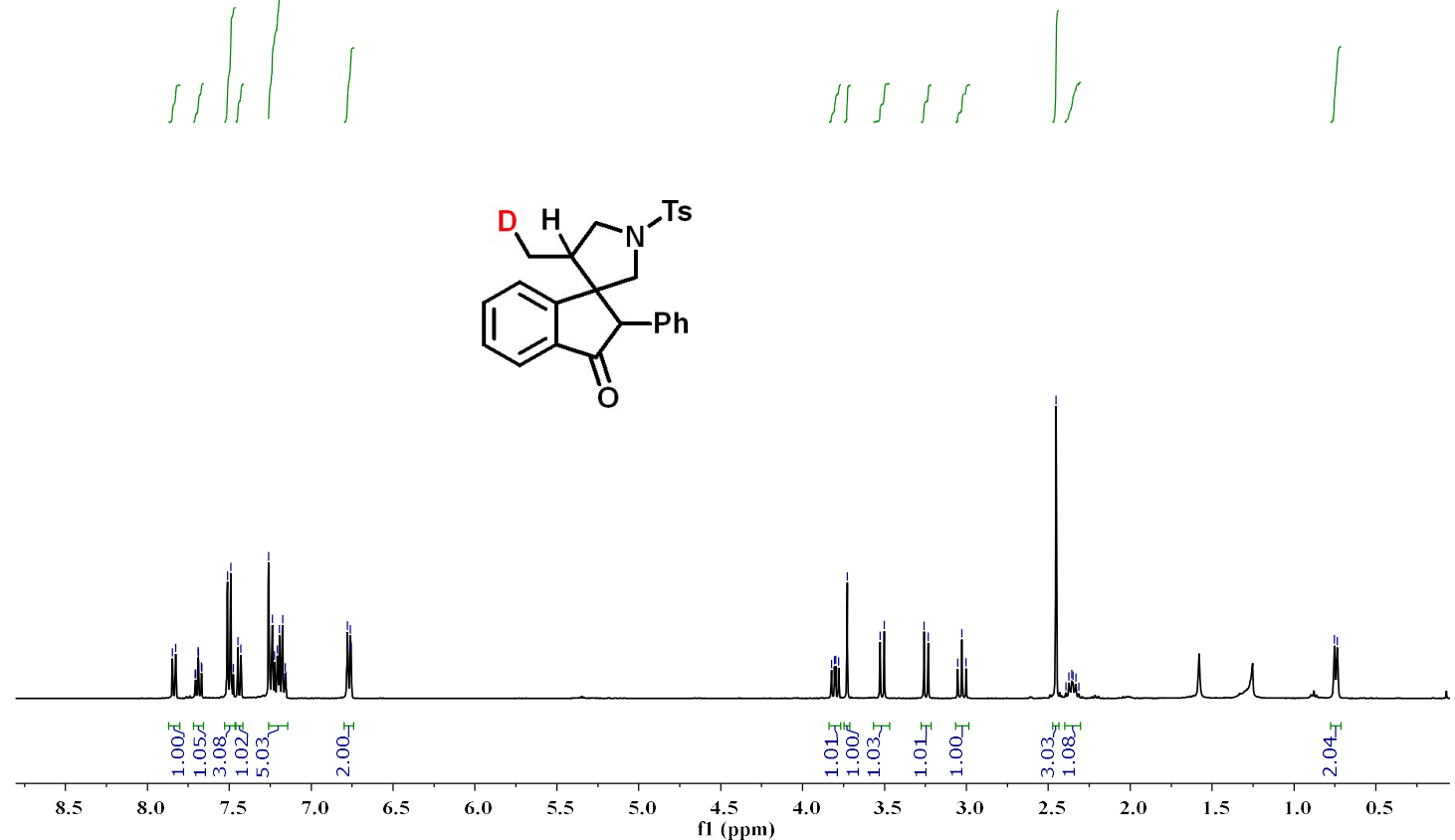
<smiles>C=CC(=O)N([Te])CC#Cc1ccccc1</smiles><smiles>O=C(O)c1ccccc1Br</smiles>

[D]-2a $(99 \%$ D)

$1 q$
$\mathrm{Ni}(\mathrm{OAc})_{2} \cdot 4 \mathrm{H}_{2} \mathrm{O}(10 \mathrm{~mol} \%)$
$\frac{\left(S, S_{p}\right){ }^{-} \text {Pr-Phosferrox (L9) }(20 \mathrm{~mol} \%)}{\text { NMP, } 100{ }^{\circ} \mathrm{C}}$

$\frac{\left(S, S_{p}\right){ }^{i} \text { Pr-Phosferrox (L9) }(20 \mathrm{~mol} \%)}{\text { NMP, } 100{ }^{\circ} \mathrm{C}}$

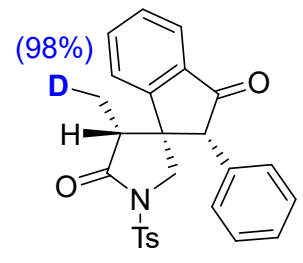

[D]-3q (50\% y, 90\% ee)

[D]-3q was prepared according to general procedure using $\mathbf{1 q}(0.1 \mathrm{mmol}, 33.9 \mathrm{mg})$ and [D]-2a ( $0.3 \mathrm{mmol}, 45.0 \mathrm{mg}$ ) and was purified by silica gel column chromatography (petroleum ether/ethyl acetate $=10 / 1 \sim 5 / 1)$ to obtain $[D]-3 q(22.3 \mathrm{mg}, 50 \%$ yield, $90 \%$ ee and $98 \% \mathrm{D}$ incorporation).
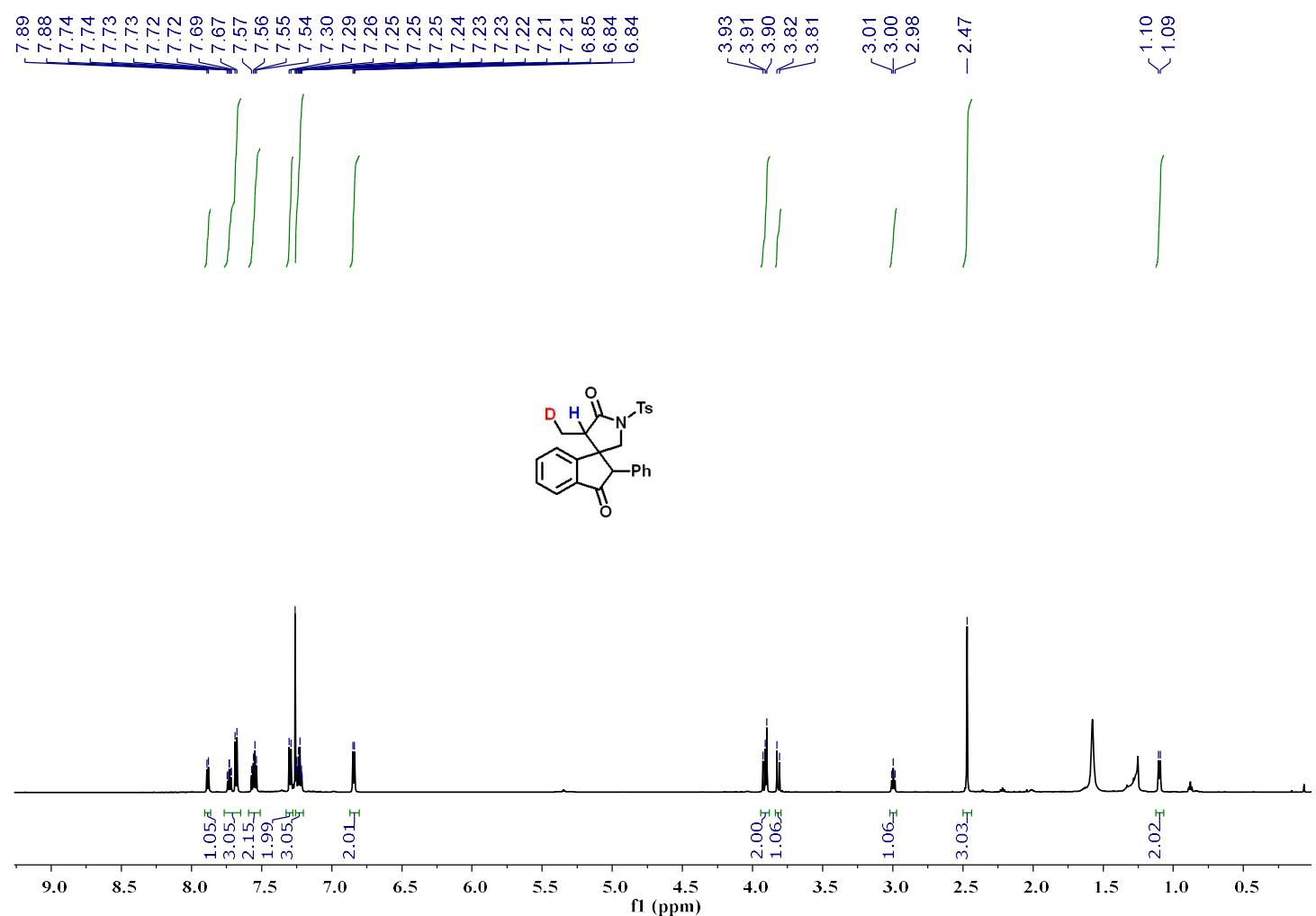


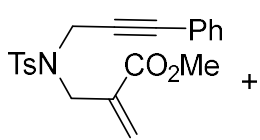

$1 \mathrm{t}$<smiles>O=C([18OH])c1ccccc1Br</smiles>

[D]-2a $(99 \%$ D)
$\mathrm{Ni}(\mathrm{OAc})_{2} \cdot 4 \mathrm{H}_{2} \mathrm{O}(10 \mathrm{~mol} \%)$ $\left(S, S_{p}\right){ }^{i}$ Pr-Phosferrox (L9) (20 mol\%) NMP, $100^{\circ} \mathrm{C}$

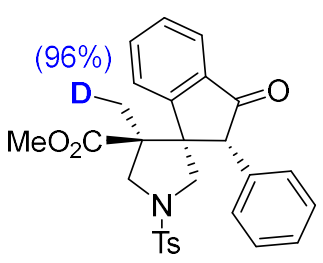

[D]-3t (69\% y, 90\% ee)

[D]-3t was prepared according to general procedure using $1 \mathrm{t}(0.1 \mathrm{mmol}, 38.3 \mathrm{mg})$ and [D]-2a (0.3 mmol, $45.3 \mathrm{mg}$ ) and was purified by silica gel column chromatography (petroleum ether/ethyl acetate $=10 / 1 \sim 5 / 1)$ to obtain $[\mathrm{D}]-3 \mathrm{t}$ (33.8 $\mathrm{mg}, 69 \%$ yield, $90 \%$ ee and $96 \% \mathrm{D}$ incorporation).
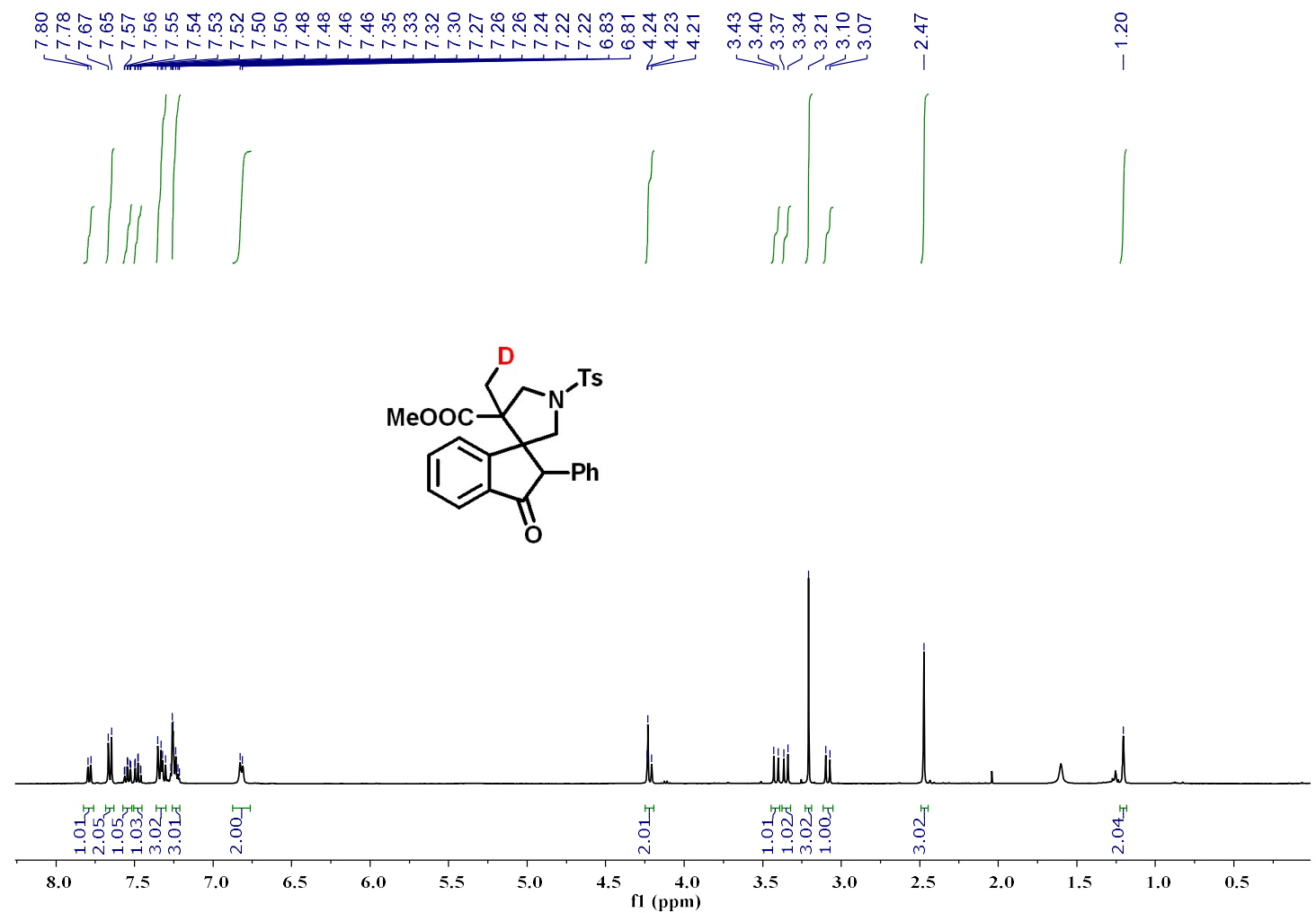
<smiles>C=C(C(=O)N([Al])CC#Cc1ccccc1)c1ccccc1</smiles>

1u<smiles>O=C(O)c1ccccc1Br</smiles>

[D]-2a (99\% D)
$\mathrm{Ni}(\mathrm{OAc})_{2} \cdot 4 \mathrm{H}_{2} \mathrm{O}(10 \mathrm{~mol} \%)$ (S, $\left.S_{p}\right)^{-}{ }^{i} \mathrm{Pr}$-Phosferrox (L9) (20 mol\%) NMP, $100^{\circ} \mathrm{C}$

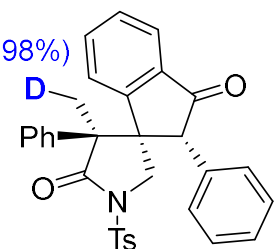

[D]-3u (78\% y, 97\% ee)

[D]-3u was prepared according to general procedure using $1 \mathbf{u}(0.1 \mathrm{mmol}, 40.1 \mathrm{mg})$ and [D]-2a ( $0.3 \mathrm{mmol}, 45.3 \mathrm{mg}$ ) and was purified by silica gel column chromatography (petroleum ether/ethyl acetate $=10 / 1 \sim 5 / 1)$ to obtain $[D]-3 u(39.6 \mathrm{mg}, 78 \%$ yield, $97 \%$ ee and $98 \% \mathrm{D}$ incorporation).
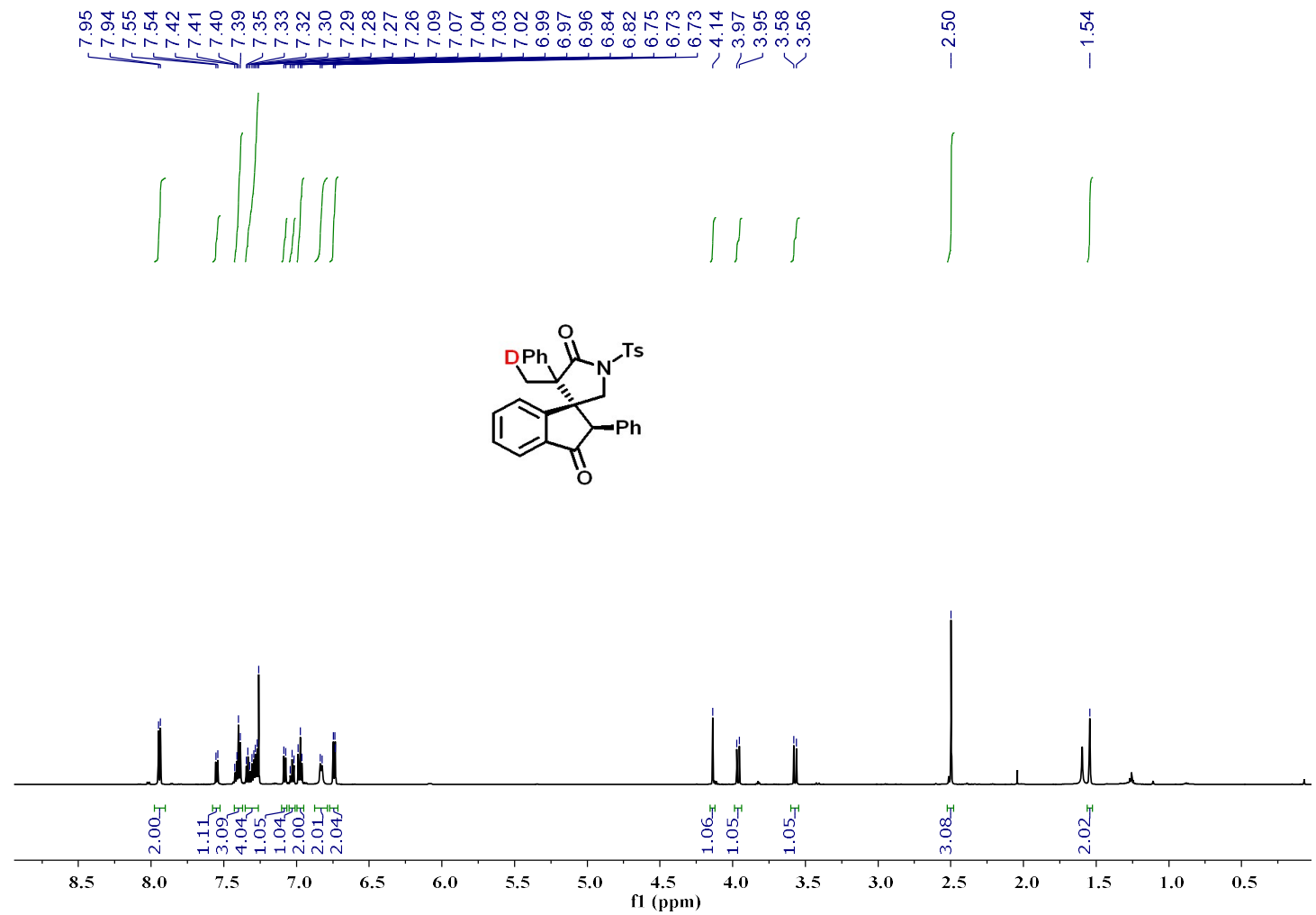


\section{Protonation with $\mathrm{D}_{2} \mathrm{O}$ :}<smiles>C=C(C)CN([Hg])CC#Cc1ccccc1</smiles>

$1 \mathrm{a}$<smiles>O=Cc1ccccc1Br</smiles>

$2 a$

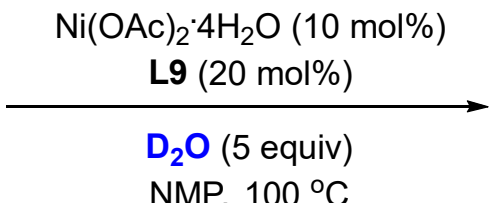

NMP, $100^{\circ} \mathrm{C}$

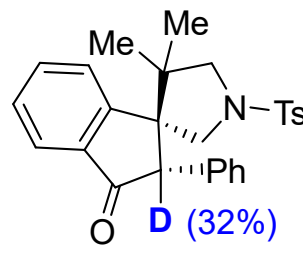

$3 a(54 \%$ y, $90 \%$ ee)

An oven-dried sealed tube equipped with a PTFE-coated stir bar was charged with $\mathrm{Ni}(\mathrm{OAc})_{2} \cdot 4 \mathrm{H}_{2} \mathrm{O}(0.01 \mathrm{mmol}, 2.5 \mathrm{mg}),(S, S p)^{-}{ }^{-} \mathrm{Pr}-\mathrm{Phosferrox}$ (L9) $(0.02 \mathrm{mmol}, 9.6 \mathrm{mg})$, (2formylphenyl)boronic acid $\mathbf{2 a}(0.3 \mathrm{mmol}, 45.0 \mathrm{mg})$ and $\mathbf{1 a}(0.1 \mathrm{mmol}, 33.9 \mathrm{mg})$. The sealed tube was evacuated and backfilled with argon (this process was repeated for three times) and then NMP $(2 \mathrm{~mL})$ and $\mathrm{D}_{2} \mathrm{O}(0.5 \mathrm{mmol}, 9 \mu \mathrm{L})$ was added. This reaction mixture was stirred at room temperature for 15 minutes and then heated at $100{ }^{\circ} \mathrm{C}$ for 36 hours until the reaction was complete (monitored by TLC). The resulting mixture was quenched with sat. $\mathrm{NH}_{4} \mathrm{Cl}$ solution (5 $\mathrm{mL})$ and further diluted with water $(10 \mathrm{~mL})$. The aqueous layer was extracted with EtOAc $(3 \mathrm{x}$ $15 \mathrm{~mL})$ and the combined organic layers were washed with brine $(2 \times 20 \mathrm{~mL})$, dried with $\mathrm{MgSO}_{4}$, filtered, and concentrated under reduced pressure. The residue was purified by chromatography on silica gel, eluting with ethyl acetate/petroleum ether 1:20 1:5 (v/v) to afford the corresponding product $3 \mathrm{a}$ ( $24.0 \mathrm{mg}, 54 \%$ yield, $90 \%$ ee and $32 \% \mathrm{D}$ incorporation).
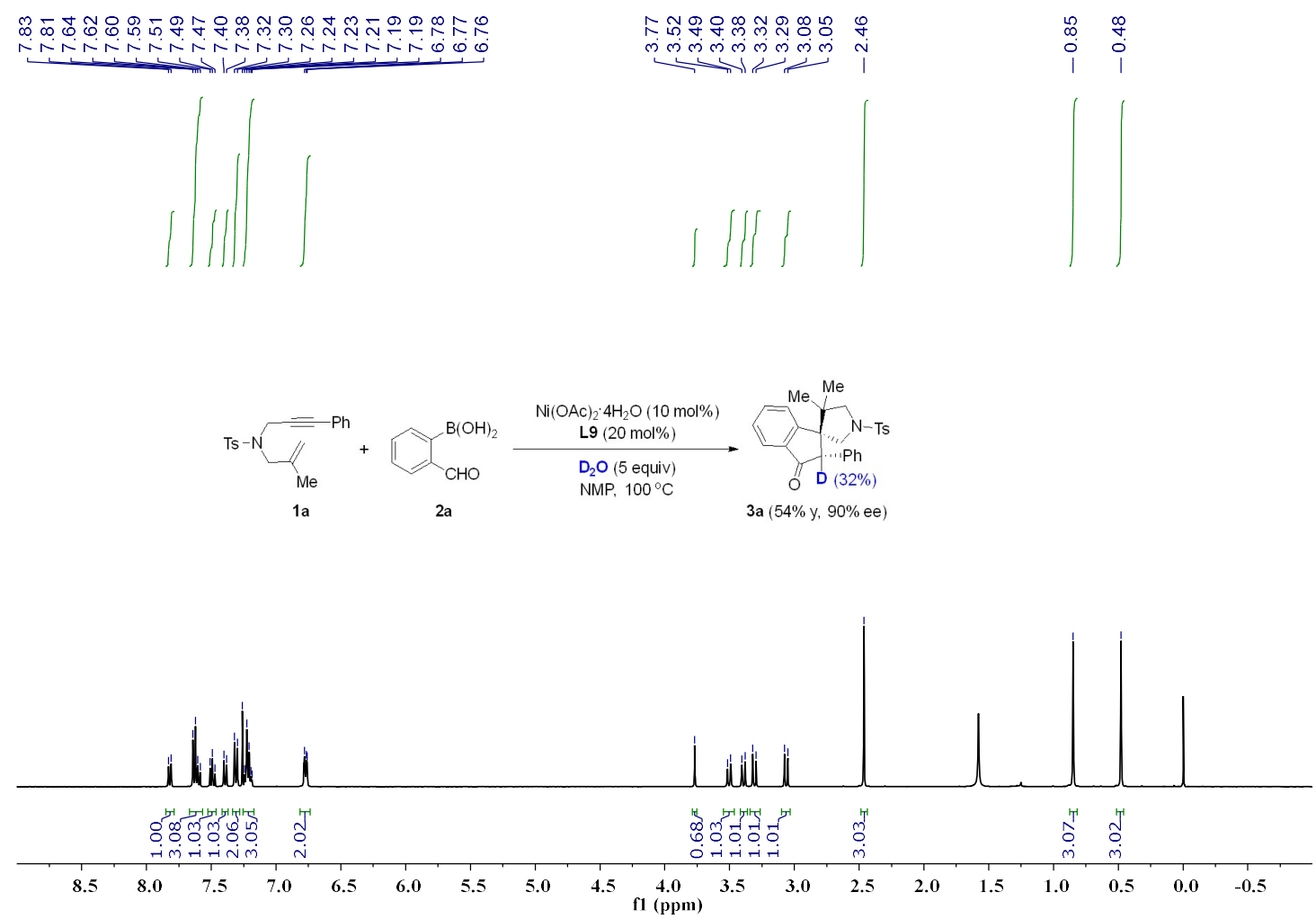


\section{Cross-over experiment:}<smiles></smiles><smiles>C=C(C)CN([AsH3])CC1=C(c2ccc(C#N)cc2)C(O)c2ccccc21</smiles>

$\mathrm{Ni}(\mathrm{OAc})_{2} \cdot 4 \mathrm{H}_{2} \mathrm{O}(10 \mathrm{~mol} \%)$

L9 (20 mol\%)

$$
\mathrm{B}(\mathrm{OH})_{3} \text { (3 equiv) }
$$

NMP, $100^{\circ} \mathrm{C}$

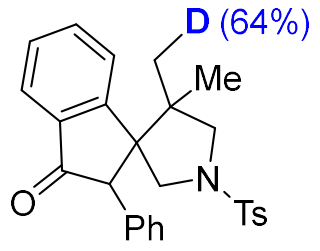

$3 a(70 \%$ y)<smiles>CCN1CC2(C)C(C(=O)c3ccccc3C2(C)C)C1c1ccc(C#N)cc1</smiles>

$3 d(70 \%$ y)

An oven-dried sealed tube equipped with a PTFE-coated stir bar was charged with $\mathrm{Ni}(\mathrm{OAc})_{2} \cdot 4 \mathrm{H}_{2} \mathrm{O}(0.01 \mathrm{mmol}, 2.5 \mathrm{mg}),(\mathrm{S}, \mathrm{Sp})-$-Pr-Phosferrox (L9) $(0.02 \mathrm{mmol}, 9.6 \mathrm{mg})$, [D]-4a' $(0.1 \mathrm{mmol}, 44.6 \mathrm{mg}), 4 \mathrm{~d}^{\prime}(0.1 \mathrm{mmol}, 47.0 \mathrm{mg})$ and $\mathrm{B}(\mathrm{OH})_{3}(0.3 \mathrm{mmol}, 18.6 \mathrm{mg})$. The sealed tube was evacuated and backfilled with argon (this process was repeated for three times) and then NMP $(2 \mathrm{~mL})$ was added. This reaction mixture was stirred at room temperature for 15 minutes and then heated at $100^{\circ} \mathrm{C}$ for 36 hours until the reaction was complete (monitored by TLC). The resulting mixture was quenched with sat. $\mathrm{NH}_{4} \mathrm{Cl}$ solution $(5 \mathrm{~mL})$ and further diluted with water $(10 \mathrm{~mL})$. The aqueous layer was extracted with EtOAc $(3 \times 15 \mathrm{~mL})$ and the combined organic layers were washed with brine $(2 \times 20 \mathrm{~mL})$, dried with $\mathrm{MgSO}_{4}$, filtered, and concentrated under reduced pressure. The residue was purified by chromatography on silica gel, eluting with ethyl acetate/petroleum ether 1:20 1:5 (v/v) to afford the corresponding product $3 a(31.0 \mathrm{mg}$, $70 \%$ yield with $64 \% \mathrm{D}$ incorporation) and $\mathbf{3 d}(32.9 \mathrm{mg}, 70 \%$ yield with $0 \% \mathrm{D}$ incorporation). 
The intermolecular competition KIE experiment:

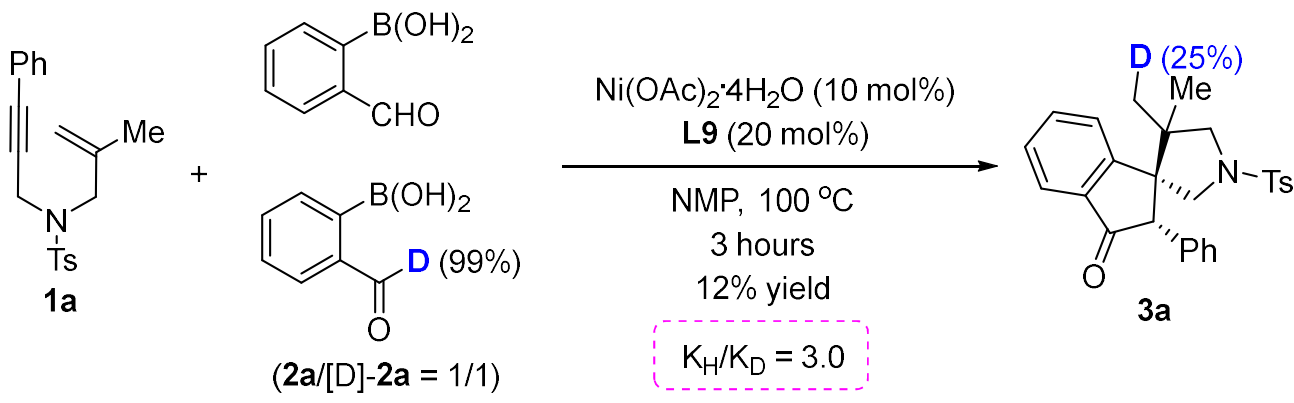

An oven-dried sealed tube equipped with a PTFE-coated stir bar was charged with $\mathrm{Ni}(\mathrm{OAc})_{2} \cdot 4 \mathrm{H}_{2} \mathrm{O}$ (0.01 mmol, $2.5 \mathrm{mg}$ ), (S, Sp)-iPr-Phosferrox (L9) (0.02 mmol, 9.6 mg), (2-(formyld)phenyl)boronic acid [D]-2a (0.15 mmol, $22.7 \mathrm{mg})$, (2-formylphenyl)boronic acid 2a $(0.15 \mathrm{mmol}$, $22.5 \mathrm{mg})$ and $1 \mathrm{a}(0.1 \mathrm{mmol}, 33.9 \mathrm{mg})$. The sealed tube was evacuated and backfilled with argon (this process was repeated for three times) and then NMP $(2 \mathrm{~mL})$ was added. This reaction mixture was stirred at room temperature for 15 minutes and then heated at $100{ }^{\circ} \mathrm{C}$ for 3 hours. The resulting mixture was quenched with sat. $\mathrm{NH}_{4} \mathrm{Cl}$ solution $(5 \mathrm{~mL})$ and further diluted with water $(10 \mathrm{~mL})$. The aqueous layer was extracted with EtOAc $(3 \times 15 \mathrm{~mL})$ and the combined organic layers were washed with brine $(2 \times 20 \mathrm{~mL})$, dried with $\mathrm{MgSO}_{4}$, filtered, and concentrated under reduced pressure. The residue was purified by chromatography on silica gel, eluting with ethyl acetate/petroleum ether 1:20 1:5 (v/v) to afford the corresponding product [D]-3a (5.4 mg, $12 \%$ yield and $25 \% \mathrm{D}$ incorporation).

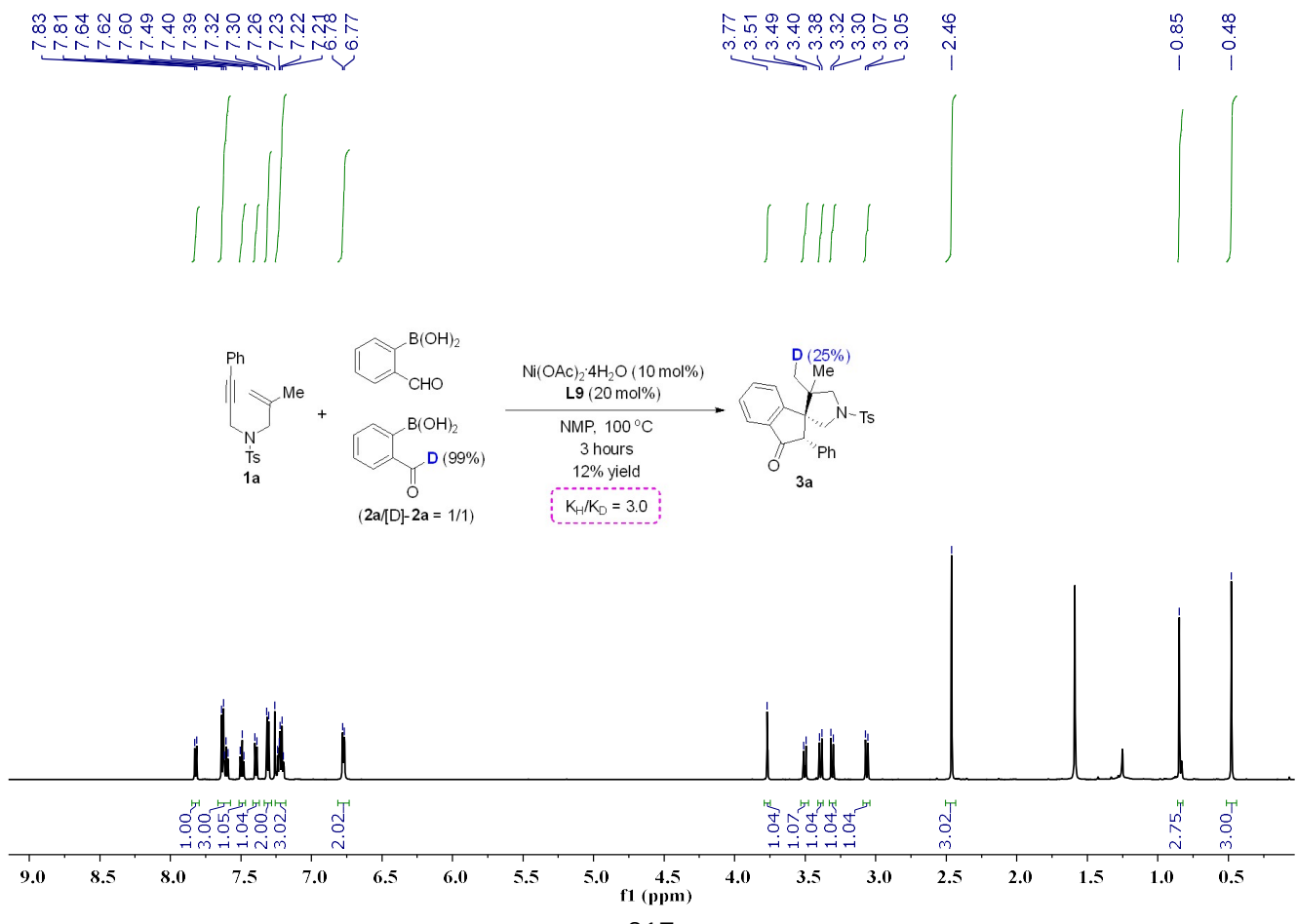




\section{Characterization data of products}

(1R,2S)-4',4'-dimethyl-2-phenyl-1'-tosylspiro[indene-1,3'-pyrrolidin]-3(2H)-one

(3a)

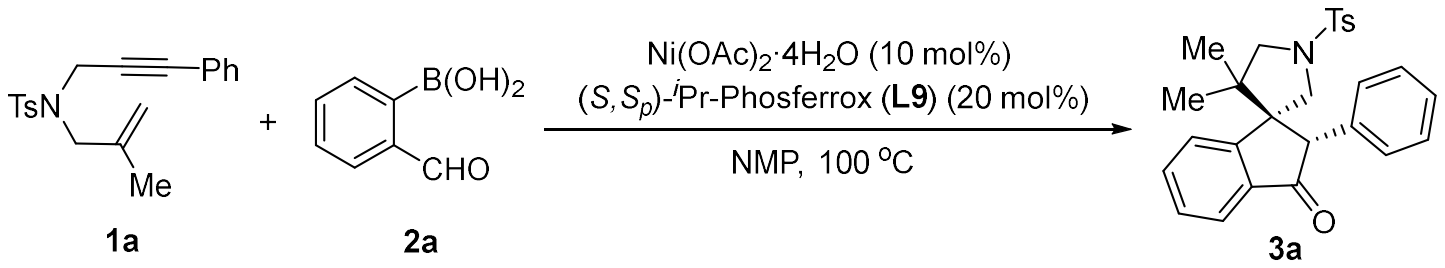

3a was prepared according to general procedure using $1 \mathbf{a}(0.1 \mathrm{mmol}, 33.9 \mathrm{mg})$ and $\mathbf{2 a}$ $(0.3 \mathrm{mmol}, 45.0 \mathrm{mg})$ and was purified by silica gel column chromatography (petroleum ether/ethyl acetate $=10 / 1 \sim 5 / 1)$ to obtain $3 \mathbf{a}(33.9 \mathrm{mg}, 76 \%$ yield $)$.

${ }^{1} \mathrm{H}$ NMR $\left(400 \mathrm{MHz}, \mathrm{CDCl}_{3}\right) \delta 7.82(\mathrm{~d}, J=7.6 \mathrm{~Hz}, 1 \mathrm{H}), 7.66-7.58(\mathrm{~m}, 3 \mathrm{H}), 7.52-7.46(\mathrm{~m}$, 1H), $7.39(\mathrm{~d}, J=7.8 \mathrm{~Hz}, 1 \mathrm{H}), 7.31(\mathrm{~d}, J=7.7 \mathrm{~Hz}, 2 \mathrm{H}), 7.25-7.18(\mathrm{~m}, 3 \mathrm{H}), 6.81-6.73(\mathrm{~m}$, 2H), 3.77 (s, 1H), 3.51 (d, J = 10.4 Hz, 1H), $3.39(\mathrm{~d}, J=10.3 \mathrm{~Hz}, 1 \mathrm{H}), 3.31$ (d, $J=10.3$ Hz, 1H), 3.06 (d, J = $10.3 \mathrm{~Hz}, 1 \mathrm{H}), 2.46$ (s, 3H), 0.85 (s, 3H), 0.48 (s, 3H);

${ }^{13} \mathrm{C}$ NMR $\left(101 \mathrm{MHz}, \mathrm{CDCl}_{3}\right) \delta 204.8,157.3,143.6,137.9,136.6,135.8,134.4,129.7$, $129.0,128.8,128.3,127.7,127.2,125.9,123.9,59.5,58.8,58.7,54.2$, 45.0, 25.0, 21.6, 21.4 ;

HRMS: (ESI) calcd for $\mathrm{C}_{27} \mathrm{H}_{28} \mathrm{NO}_{3} \mathrm{~S}^{+}[\mathrm{M}+\mathrm{H}]^{+} 446.1784$; found 446.1779 .

The enantiomeric purity was established by HPLC analysis using a chiral column: OD$\mathrm{H}$ column, $30^{\circ} \mathrm{C}, n$-Hexane $/ i$-Propanol $=80 / 20$ as eluent, $254 \mathrm{~nm}, 1 \mathrm{~mL} / \mathrm{min}$. tR $=8 \mathrm{~min}$ (major), 12 min (minor).

Optical Rotation: $[\alpha]_{D}{ }^{28}-51.6$ (c 5.0, MeCN) for $93 \%$ ee.

Absolute stereochemistry was determined through X-ray crystallographic analysis. 
$<$ Chromatogram $>$

mAU

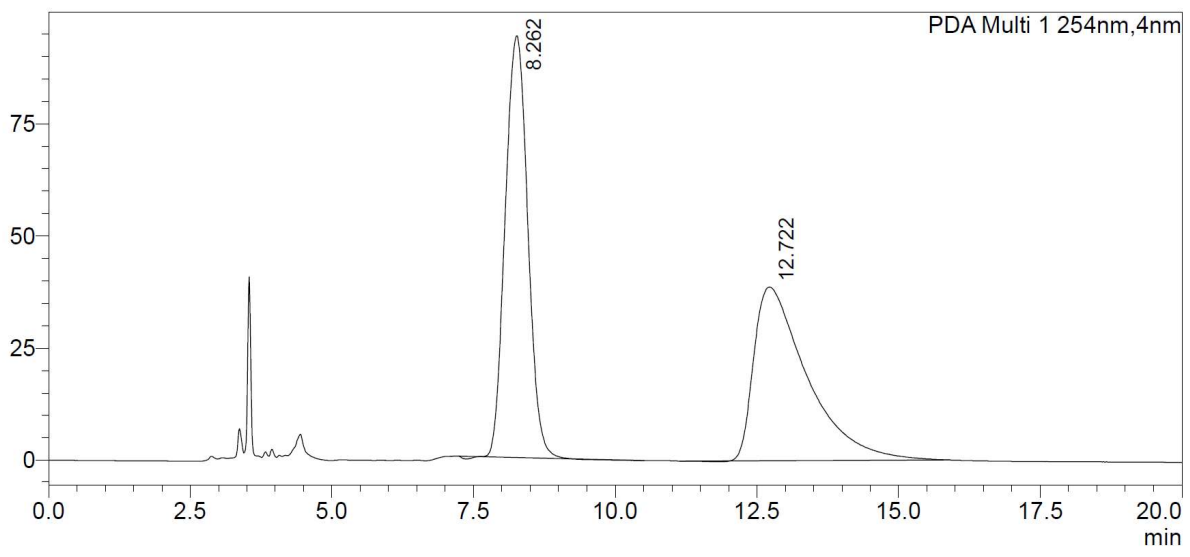

<Peak Table>

PDA Ch1 254nm

\begin{tabular}{|r|r|r|r|r|r|r|r|}
\hline Peak\# Ret. Time & Area & Height & Conc. & Unit & Mark & Name \\
\hline 1 & 8.262 & 2591834 & 94010 & 0.000 & & $\mathrm{M}$ & \\
\hline 2 & 12.722 & 2536482 & 38721 & 0.000 & & $\mathrm{M}$ & \\
\hline Total & & 5128316 & 132731 & & & & \\
\hline
\end{tabular}

<Chromatogram>

mAU

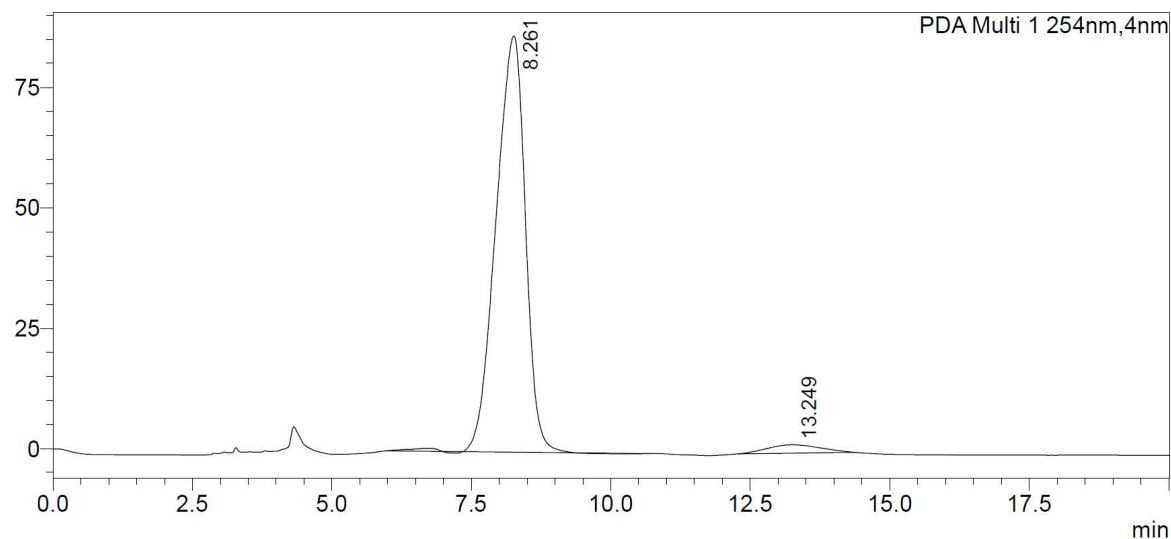

<Peak Table>

PDA Ch1 254nm

\begin{tabular}{|r|r|r|r|r|r|r|r|}
\hline Peak\# Ret. Time & \multicolumn{1}{|c|}{ Area } & \multicolumn{1}{|c|}{ Height } & Conc. & Unit & Mark & Name \\
\hline 1 & 8.261 & 3172964 & 86375 & 0.000 & & $\mathrm{M}$ & \\
\hline 2 & 13.249 & 115765 & 1740 & 0.000 & & $\mathrm{M}$ & \\
\hline Total & & 3288730 & 88114 & & & & \\
\hline
\end{tabular}


(1R,2S)-2-(4-methoxyphenyl)-4',4'-dimethyl-1'-tosylspiro[indene-1,3'-pyrrolidin]$3(2 H)$-one (3b)<smiles>C=C(C)CN(S)CC#Cc1ccc(OC)cc1</smiles>

$1 b$<smiles>O=Cc1ccccc1Br</smiles>

2a

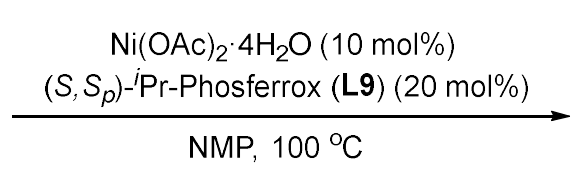

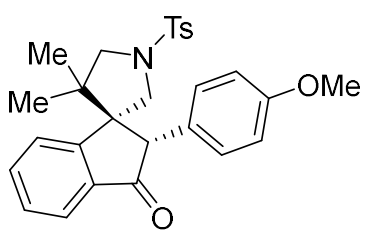

$3 b$

3b was prepared according to general procedure using $1 \mathbf{a}(0.1 \mathrm{mmol}, 36.9 \mathrm{mg})$ and 2a $(0.3 \mathrm{mmol}, 45.0 \mathrm{mg})$ and was purified by silica gel column chromatography (petroleum ether/ethyl acetate $=10 / 1 \sim 5 / 1)$ to obtain $3 \mathbf{b}(40.9 \mathrm{mg}, 86 \%$ yield).

${ }^{1} \mathrm{H} \mathrm{NMR}\left(400 \mathrm{MHz}, \mathrm{CDCl}_{3}\right) \delta 7.81(\mathrm{~d}, J=7.5 \mathrm{~Hz}, 1 \mathrm{H}), 7.67-7.63(\mathrm{~m}, 2 \mathrm{H}), 7.62-7.57(\mathrm{~m}$, 1H), 7.52-7.45 (m, 1H), 7.37 (d, J = 7.8 Hz, 1H), 7.32 (d, J = 8.0 Hz, 2H), 6.76-6.66 (m, 4H), $3.77(\mathrm{~s}, 3 \mathrm{H}), 3.72(\mathrm{~s}, 1 \mathrm{H}), 3.49(\mathrm{~d}, J=10.4 \mathrm{~Hz}, 1 \mathrm{H}), 3.38(\mathrm{~d}, J=10.3 \mathrm{~Hz}, 1 \mathrm{H})$, 3.30 (d, $J=10.3 \mathrm{~Hz}, 1 \mathrm{H}$ ), 3.09 (d, J = 10.3 Hz, 1H), 2.46 (s, 3H), 0.83 (s, 3H), 0.46 (s, $3 \mathrm{H})$;

${ }^{13} \mathrm{C} \mathrm{NMR}\left(151 \mathrm{MHz}, \mathrm{CDCl}_{3}\right) \delta$ 205.2, 158.9, 157.3, 143.6, 136.6, 135.7, 134.4, 130.0, $129.8,129.3,128.8,127.2,125.9,123.9,114.4,59.5,58.9,58.0,55.3,54.2,44.9,25.0$, 21.6, 21.5;

HRMS: (ESI) calcd for $\mathrm{C}_{28} \mathrm{H}_{30} \mathrm{NO}_{4} \mathrm{~S}^{+}[\mathrm{M}+\mathrm{H}]^{+}$476.1890; found 476.1886.

The enantiomeric purity was established by HPLC analysis using a chiral column: OD$\mathrm{H}$ column, $30^{\circ} \mathrm{C}, n$-Hexane $/ \mathrm{i}$-Propanol $=80 / 20$ as eluent, $254 \mathrm{~nm}, 1 \mathrm{~mL} / \mathrm{min} . \mathrm{tR}=11$ $\min$ (major), $16 \min$ (minor).

Optical Rotation: $[\alpha]_{D}^{28}-13.8$ (c 5.0, MeCN) for $99 \%$ ee.

Absolute stereochemistry was determined through analogy with $\mathbf{3 a}$. 
<Chromatogram>

mAU

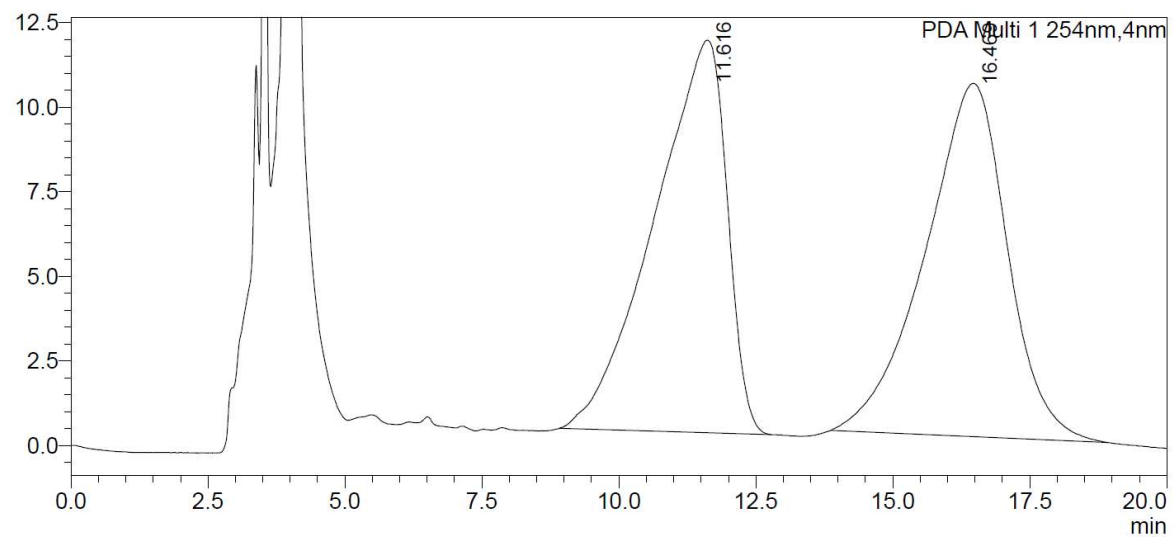

<Peak Table>

PDA Ch1 254nm

\begin{tabular}{|r|r|r|r|r|r|r|r|}
\hline Peak\# Ret. Time & Area & Height & \multicolumn{1}{|c|}{ Conc. } & Unit & Mark & Name \\
\hline 1 & 11.616 & 1072153 & 11598 & 0.000 & & & \\
\hline 2 & 16.469 & 1089709 & 10439 & 0.000 & & & \\
\hline Total & & 2161862 & 22036 & & & & \\
\hline
\end{tabular}

\section{<Chromatogram>}

mAU

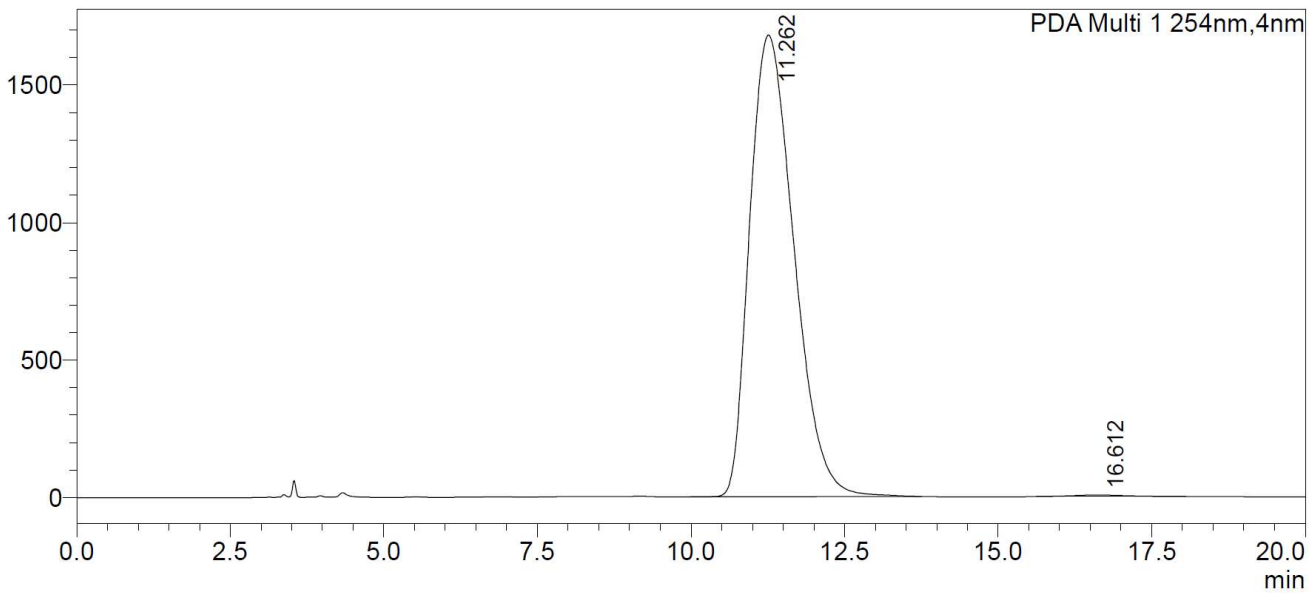

<Peak Table>

PDA Ch1 254nm

Peak\# Ret. Time

\begin{tabular}{r|r|}
\hline 1 & 11.262 \\
\hline 2 & 16.612 \\
\hline Total &
\end{tabular}

\begin{tabular}{|r|r|r|r|r|r|}
\hline \multicolumn{1}{|c|}{ Area } & Height & \multicolumn{1}{c|}{ Conc. } & Unit & Mark & Name \\
\hline 84038641 & 1678411 & 0.000 & & $\mathrm{M}$ & \\
\hline 3435064 & 5269 & 0.000 & & $\mathrm{M}$ & \\
\hline 843704 & 1683680 & & & & \\
\hline
\end{tabular}


(1R,2S)-2-(4-(dimethylamino)phenyl)-4',4'-dimethyl-1'-tosylspiro[indene-1,3'pyrrolidin]-3(2H)-one (3c)

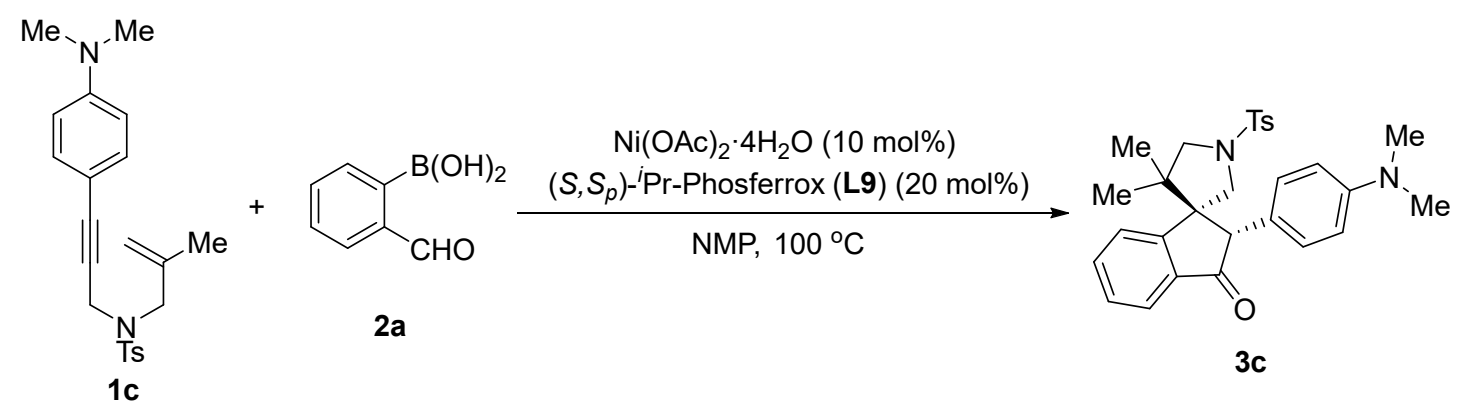

3c was prepared according to general procedure using $1 \mathrm{c}(0.1 \mathrm{mmol}, 38.2 \mathrm{mg})$ and $2 \mathrm{a}$ $(0.3 \mathrm{mmol}, 45.0 \mathrm{mg})$ and was purified by silica gel column chromatography (petroleum ether/ethyl acetate $=10 / 1 \sim 5 / 1)$ to obtain $3 \mathrm{c}(36.1 \mathrm{mg}, 74 \%$ yield $)$.

${ }^{1} \mathrm{H} \mathrm{NMR}\left(400 \mathrm{MHz}, \mathrm{CDCl}_{3}\right)$ ठ 7.82-7.78 (m, 1H), 7.69-7.64 (m, 2H), 7.60-7.54 (m, 1H), 7.49-7.43 (m, 1H), 7.35 (d, J = 7.8 Hz, 1H), 7.34-7.30 (m, 2H), 6.65-6.59 (m, 2H), 6.58$6.51(\mathrm{~m}, 2 \mathrm{H}), 3.68(\mathrm{~s}, 1 \mathrm{H}), 3.49(\mathrm{~d}, J=10.3 \mathrm{~Hz}, 1 \mathrm{H}), 3.41(\mathrm{~d}, J=10.5 \mathrm{~Hz}, 1 \mathrm{H}), 3.29$ (d, $J=10.3 \mathrm{~Hz}, 1 \mathrm{H}$ ), 3.17 (d, $J=10.4 \mathrm{~Hz}, 1 \mathrm{H}), 2.91(\mathrm{~s}, 6 \mathrm{H}), 2.46(\mathrm{~s}, 3 \mathrm{H}), 0.85(\mathrm{~s}, 3 \mathrm{H})$, $0.45(\mathrm{~s}, 3 \mathrm{H})$;

${ }^{13} \mathrm{C}$ NMR $\left(151 \mathrm{MHz}, \mathrm{CDCl}_{3}\right) \delta 205.5,157.4,143.5,136.7,135.5,134.5,129.7,128.8$, 128.6, 127.2, 125.8, 123.8, 112.9, 59.5, 59.0, 58.0, 54.2, 44.8, 40.5, 25.0, 21.6, 21.4; HRMS: (ESI) calcd for $\mathrm{C}_{29} \mathrm{H}_{33} \mathrm{~N}_{2} \mathrm{O}_{3} \mathrm{~S}^{+}[\mathrm{M}+\mathrm{H}]^{+}$489.2206; found 489.2208.

The enantiomeric purity was established by HPLC analysis using a chiral column: OD$\mathrm{H}$ column, $30^{\circ} \mathrm{C}, n$-Hexane/ $i$-Propanol $=80 / 20$ as eluent, $254 \mathrm{~nm}, 1 \mathrm{~mL} / \mathrm{min} . \mathrm{tR}=12$ $\min$ (major), 18 min (minor).

Optical Rotation: $[\alpha]_{D}{ }^{28}-51.6$ (c 5.0, MeCN) for $98 \%$ ee.

Absolute stereochemistry was determined through analogy with $\mathbf{3 a}$. 
<Chromatogram>

mAU

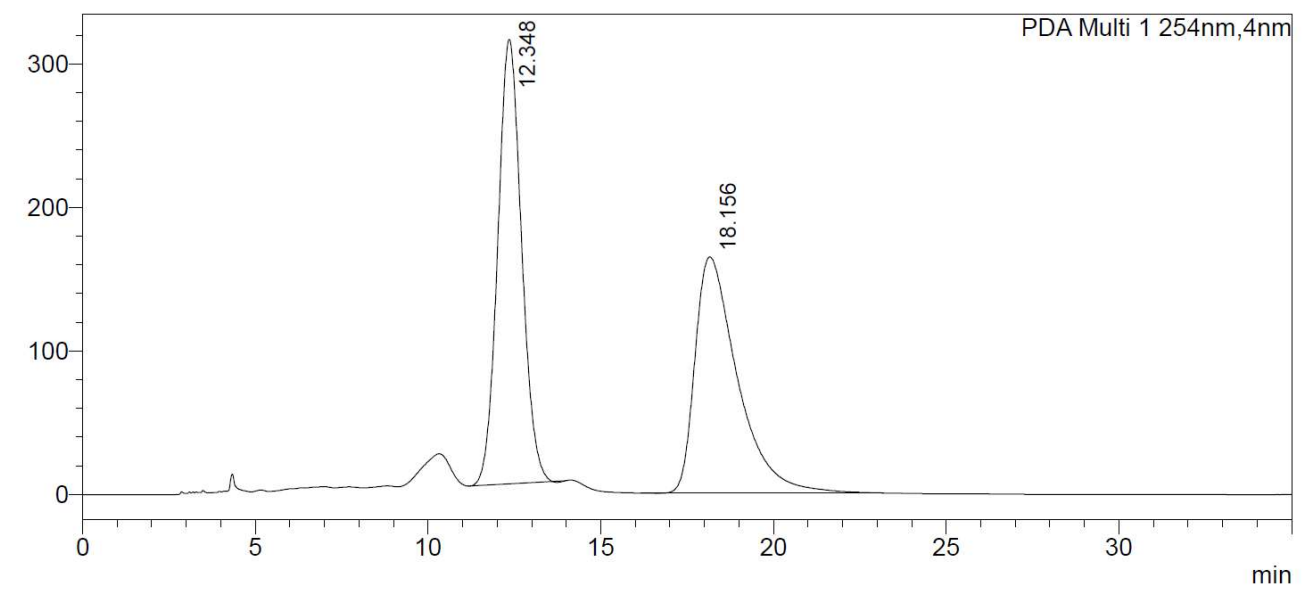

<Peak Table>

PDA Ch1 254nm

\begin{tabular}{|c|c|c|c|c|c|c|c|}
\hline Peak\# & Ret. Time & Area & Height & Conc. & Unit & Mark & Name \\
\hline 1 & 12.348 & 14828504 & 309533 & 0.000 & & M & \\
\hline 2 & 18.156 & 14072221 & 164451 & 0.000 & & M & \\
\hline Total & & 28900725 & 473984 & & & & \\
\hline
\end{tabular}

\section{<Chromatogram>}

mAU

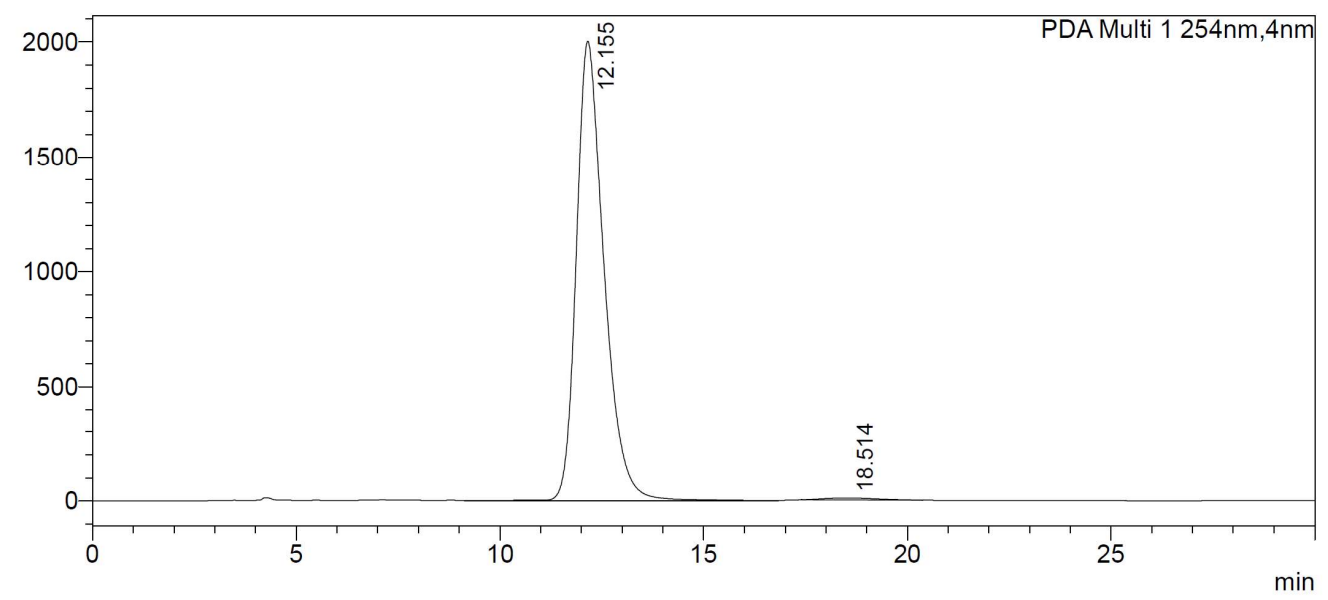

<Peak Table>

PDA Ch1 254nm

\begin{tabular}{|r|r|r|r|r|r|r|r|}
\hline Peak\# Ret. Time & \multicolumn{1}{|c|}{ Area } & Height & Conc. & Unit & Mark & Name \\
\hline 1 & 12.155 & 94474885 & 2001598 & 0.000 & & $M$ & \\
\hline 2 & 18.514 & 803362 & 8458 & 0.000 & & $M$ & \\
\hline Total & & 95278247 & 2010056 & & & & \\
\hline
\end{tabular}


4-((1R,2S)-4',4'-dimethyl-3-oxo-1'-tosyl-2,3-dihydrospiro[indene-1,3'-pyrrolidin]2-yl)benzonitrile (3d)

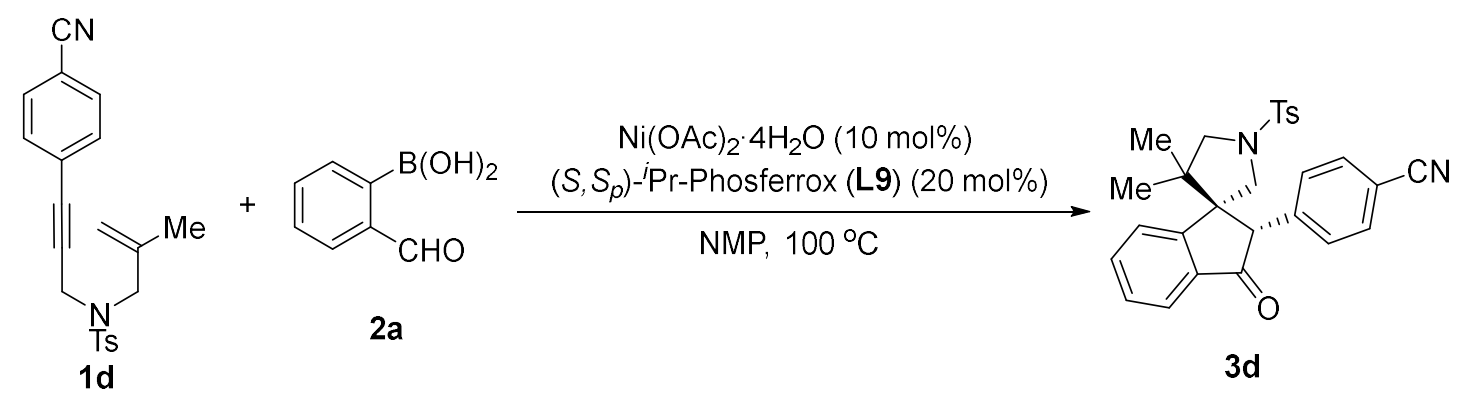

3d was prepared according to general procedure using $1 \mathbf{d}(0.1 \mathrm{mmol}, 36.4 \mathrm{mg})$ and 2a $(0.3 \mathrm{mmol}, 45.0 \mathrm{mg})$ and was purified by silica gel column chromatography (petroleum ether/ethyl acetate $=10 / 1 \sim 5 / 1)$ to obtain $3 \mathrm{~d}(36.2 \mathrm{mg}, 77 \%$ yield $)$.

${ }^{1} \mathrm{H} \mathrm{NMR}\left(400 \mathrm{MHz}, \mathrm{CDCl}_{3}\right) \delta 7.82(\mathrm{~d}, J=7.6 \mathrm{~Hz}, 1 \mathrm{H}), 7.67-7.59(\mathrm{~m}, 3 \mathrm{H}), 7.55-7.47(\mathrm{~m}$, 3H), 7.40 (d, J = 7.8 Hz, 1H), 7.32 (d, J = 7.8 Hz, 2H), 6.91 (d, J=7.8 Hz, 2H), 3.83 (s, $1 \mathrm{H}), 3.51(\mathrm{~d}, J=10.4 \mathrm{~Hz}, 1 \mathrm{H}), 3.40(\mathrm{~d}, J=10.2 \mathrm{~Hz}, 1 \mathrm{H}), 3.33(\mathrm{~d}, J=10.4 \mathrm{~Hz}, 1 \mathrm{H})$, 2.97 (d, J = 10.2 Hz, 1H), 2.47 (s, 3H), 0.86 (s, 3H), 0.50 (s, 3H);

${ }^{13} \mathrm{C} \mathrm{NMR}\left(151 \mathrm{MHz}, \mathrm{CDCl}_{3}\right) \delta$ 203.2, 156.6, 143.9, 143.3, 136.2, 136.2, 134.3, 132.7, $129.8,129.2,129.2,127.1,125.9,124.2,118.2,111.7,59.2,58.8,53.6,45.3,24.7$, 21.6, 21.5;

HRMS: (ESI) calcd for $\mathrm{C}_{28} \mathrm{H}_{27} \mathrm{~N}_{2} \mathrm{O}_{3} \mathrm{~S}^{+}[\mathrm{M}+\mathrm{H}]^{+}$471.1737; found 471.1753 .

The enantiomeric purity was established by HPLC analysis using a chiral column: OD$\mathrm{H}$ column, $30^{\circ} \mathrm{C}, n$-Hexane/i-Propanol $=80 / 20$ as eluent, $254 \mathrm{~nm}, 1 \mathrm{~mL} / \mathrm{min}$. $\mathrm{tR}=14$ $\min$ (major), 25 min (minor).

Optical Rotation: $[\alpha]_{D}^{28}-116.1$ (c 5.0, MeCN) for $98 \%$ ee.

Absolute stereochemistry was determined through analogy with $\mathbf{3 a}$. 
<Chromatogram>

mAU

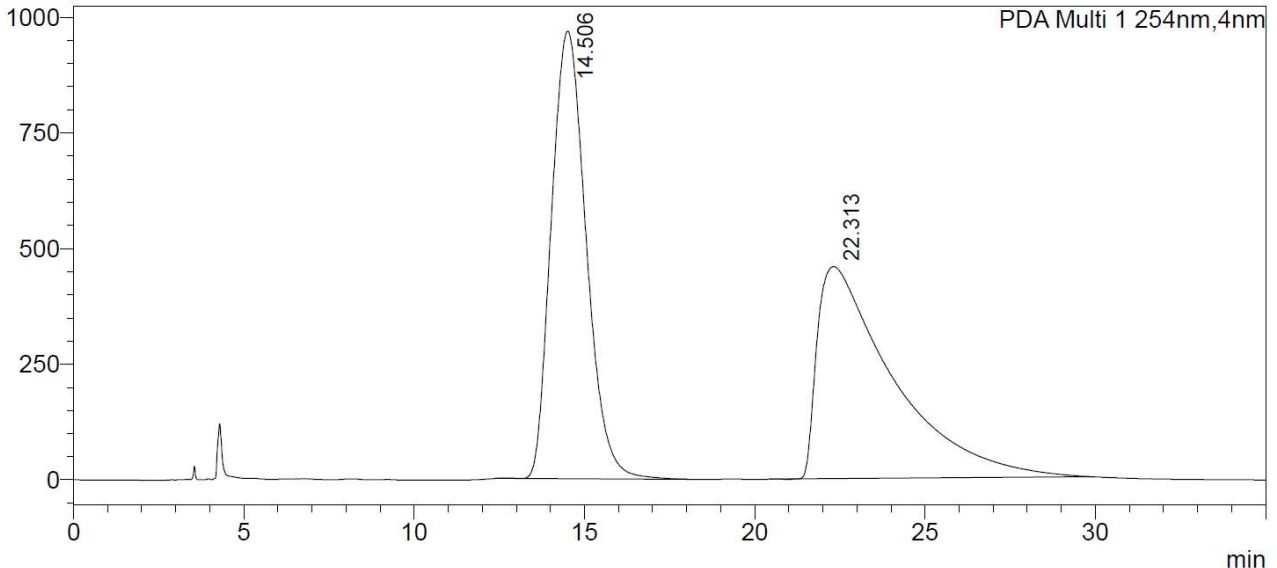

<Peak Table>

PDA Ch1 254nm

\begin{tabular}{|r|r|r|r|r|r|r|r|}
\hline Peak\# Ret. Time & \multicolumn{1}{|c|}{ Area } & Height & Conc. & Unit & Mark & Name \\
\hline 1 & 14.506 & 68804754 & 967982 & 0.000 & & $\mathrm{M}$ & \\
\hline 2 & 22.313 & 71208502 & 458690 & 0.000 & & $\mathrm{M}$ & \\
\hline Total & & 140013257 & 1426672 & & & & \\
\end{tabular}

<Chromatogram>

mAU

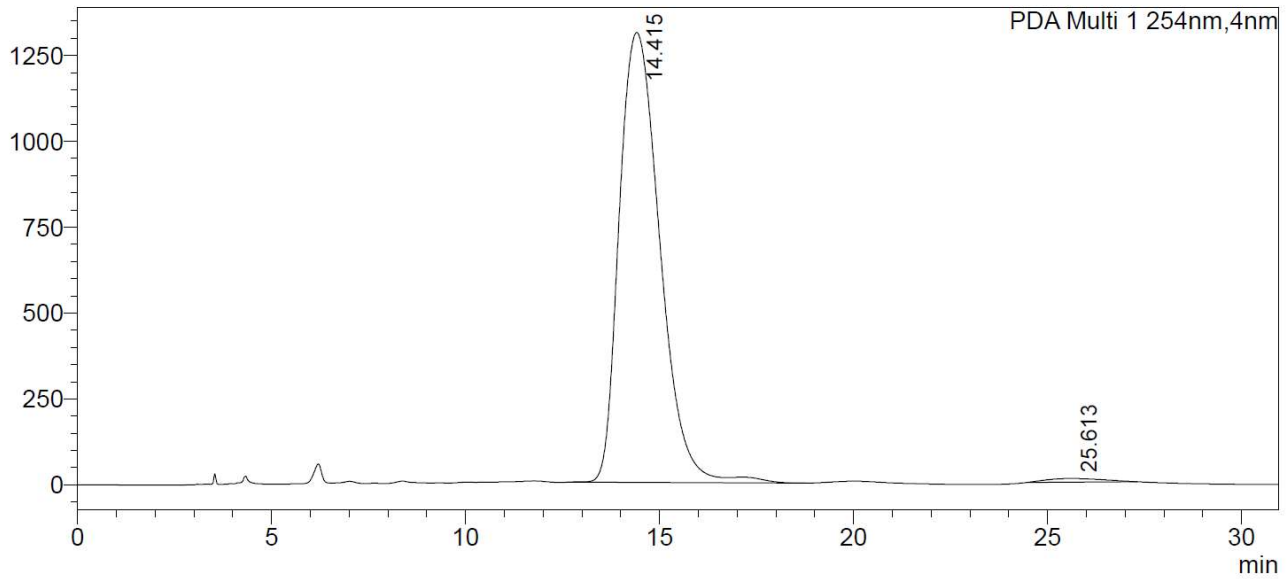

<Peak Table>

PDA Ch1 254nm

\begin{tabular}{|r|r|r|r|r|r|r|r|}
\hline Peak\# Ret. Time & \multicolumn{1}{|c|}{ Area } & Height & Conc. & Unit & Mark & Name \\
\hline 1 & 14.415 & 95685364 & 1310222 & 0.000 & & $\mathrm{M}$ & \\
\hline 2 & 25.613 & 1061426 & 10549 & 0.000 & & $\mathrm{M}$ & \\
\hline Total & & 96746790 & 1320771 & & & & \\
\hline
\end{tabular}


(1R,2S)-2-(4-chlorophenyl)-4',4'-dimethyl-1'-tosylspiro[indene-1,3'-pyrrolidin]$3(2 H)$-one (3e)

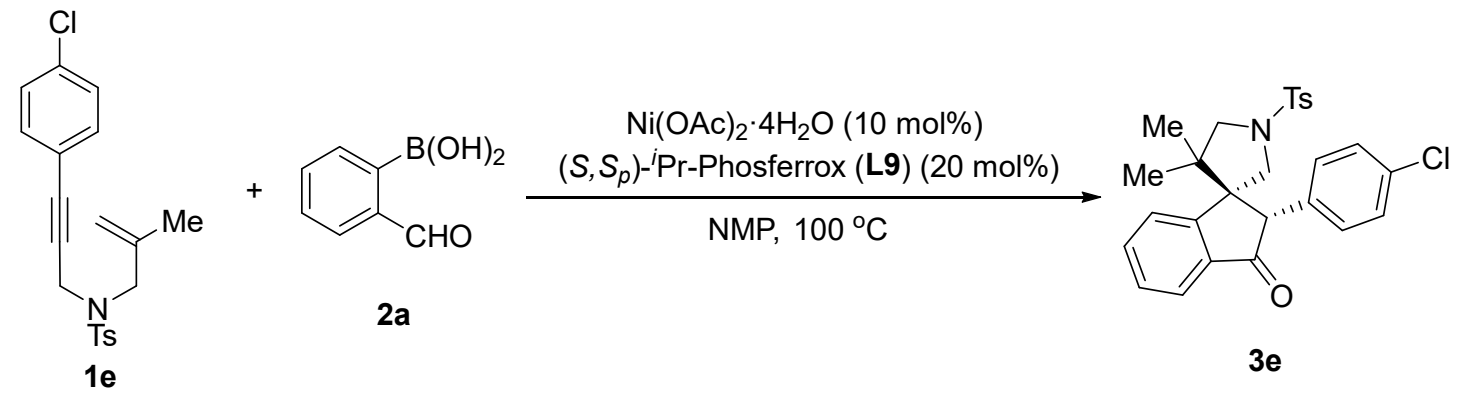

3e was prepared according to general procedure using $1 \mathbf{e}(0.1 \mathrm{mmol}, 37.4 \mathrm{mg})$ and $2 \mathbf{a}$ $(0.3 \mathrm{mmol}, 45.0 \mathrm{mg})$ and was purified by silica gel column chromatography (petroleum ether/ethyl acetate $=10 / 1 \sim 5 / 1)$ to obtain $3 \mathbf{e}(38.3 \mathrm{mg}, 89 \%$ yield $)$.

${ }^{1} \mathrm{H} \mathrm{NMR}\left(400 \mathrm{MHz}, \mathrm{CDCl}_{3}\right) \delta 7.80(\mathrm{~d}, J=7.5 \mathrm{~Hz}, 1 \mathrm{H}), 7.65-7.62(\mathrm{~m}, 2 \mathrm{H}), 7.61-7.58(\mathrm{~m}$, 1H), 7.51-7.45 (m, 1H), 7.38 (d, $J=7.8 \mathrm{~Hz}, 1 \mathrm{H}), 7.31(\mathrm{~d}, J=7.8 \mathrm{~Hz}, 2 \mathrm{H}), 7.17(\mathrm{~d}, J=$ $8.7 \mathrm{~Hz}, 1 \mathrm{H}), 6.70$ (d, J = 8.3 Hz, 2H), $3.74(\mathrm{~s}, 1 \mathrm{H}), 3.50$ (d, J = $10.4 \mathrm{~Hz}, 1 \mathrm{H}), 3.39$ (d, J $=10.3 \mathrm{~Hz}, 1 \mathrm{H}), 3.31(\mathrm{~d}, J=10.4 \mathrm{~Hz}, 1 \mathrm{H}), 3.03(\mathrm{~d}, J=10.3 \mathrm{~Hz}, 1 \mathrm{H}), 2.45(\mathrm{~s}, 3 \mathrm{H}), 0.83$ (s, 3H), $0.43(\mathrm{~s}, 3 \mathrm{H})$;

${ }^{13} \mathrm{C}$ NMR $\left(151 \mathrm{MHz}, \mathrm{CDCl}_{3}\right) \delta 204.1,156.8,143.7,136.4,136.3,135.9,134.2,133.5$, $129.7,129.6,129.1,128.9,127.1,125.9,124.0,59.3,58.7,58.1,53.8,45.1,24.7,21.5$, 21.5 .

HRMS: (ESI) calcd for $\mathrm{C}_{27} \mathrm{H}_{27} \mathrm{NO}_{3} \mathrm{CIS}^{+}[\mathrm{M}+\mathrm{H}]^{+}$480.1395; found 480.1380.

The enantiomeric purity was established by HPLC analysis using a chiral column: OD$\mathrm{H}$ column, $30^{\circ} \mathrm{C}, n$-Hexane/ $i$-Propanol $=80 / 20$ as eluent, $254 \mathrm{~nm}, 1 \mathrm{~mL} / \mathrm{min}$. $\mathrm{tR}=7 \mathrm{~min}$ (major), 10 min (minor).

Optical Rotation: $[\alpha]_{D}^{28}-92.0$ (c 5.0, MeCN) for 95\% ee.

Absolute stereochemistry was determined through analogy with $\mathbf{3 a}$. 


\section{<Chromatogram>}

mAU

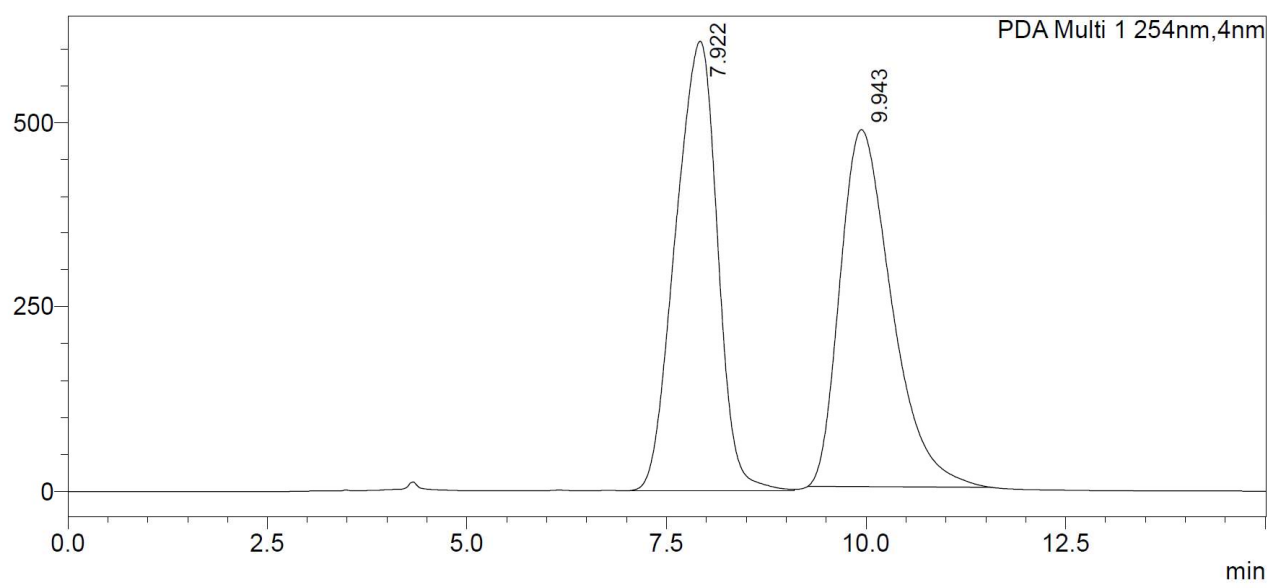

<Peak Table>

PDA Ch1 254nm

\begin{tabular}{|r|r|r|r|r|r|r|r|}
\hline Peak\# Ret. Time & \multicolumn{1}{|c|}{ Area } & Height & Conc. & Unit & Mark & Name \\
\hline 1 & 7.922 & 22306538 & 609744 & 0.000 & & & \\
\hline 2 & 9.943 & 21819578 & 484108 & 0.000 & & $\mathrm{M}$ & \\
\hline Total & & 44126116 & 1093852 & & & & \\
\hline
\end{tabular}

<Chromatogram>

mAU

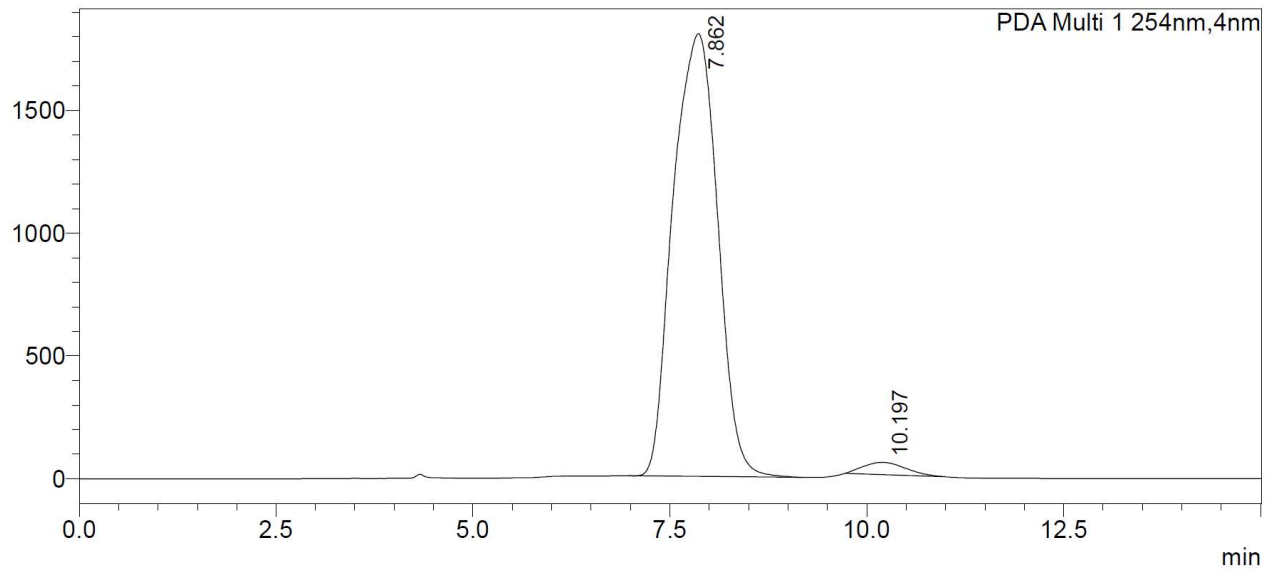

<Peak Table>

PDA Ch1 254nm

\begin{tabular}{|r|r|r|r|r|r|r|r|}
\hline Peak\# Ret. Time & \multicolumn{1}{|c|}{ Area } & Height & Conc. & Unit & Mark & Name \\
\hline 1 & 7.862 & 70880806 & 1802650 & 0.000 & & $\mathrm{M}$ & \\
\hline 2 & 10.197 & 1848628 & 49592 & 0.000 & & $\mathrm{M}$ & \\
\hline Total & & 72729433 & 1852242 & & & & \\
\hline
\end{tabular}


(1R,2S)-4',4'-dimethyl-1'-tosyl-2-(4-(trifluoromethyl)phenyl)spiro[indene-1,3'pyrrolidin]-3(2H)-one (3f)

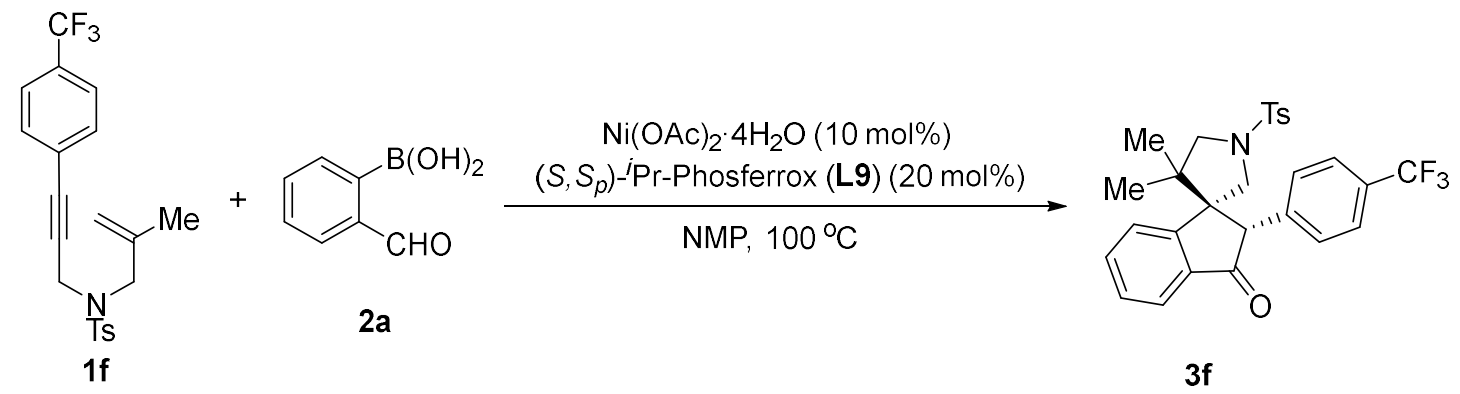

3f was prepared according to general procedure using $1 \mathbf{1 f}(0.1 \mathrm{mmol}, 40.7 \mathrm{mg})$ and $\mathbf{2 a}$ $(0.3 \mathrm{mmol}, 45.0 \mathrm{mg})$ and was purified by silica gel column chromatography (petroleum ether/ethyl acetate $=10 / 1 \sim 5 / 1)$ to obtain $3 \mathbf{f}(35.9 \mathrm{mg}, 70 \%$ yield $)$.

${ }^{1} \mathrm{H}$ NMR $\left(400 \mathrm{MHz}, \mathrm{CDCl}_{3}\right) \delta$ 7.85-7.81 (m, 1H), 7.66-7.61 (m, 3H), 7.52 (td, J = 7.5, 0.9 Hz, 1H), 7.49-7.45 (m, 2H), 7.42-7.39 (m, 1H), 7.33-7.29 (m, 2H), 6.90 (d, J = 8.0 $\mathrm{Hz}, 2 \mathrm{H}), 3.83(\mathrm{~s}, 1 \mathrm{H}), 3.52(\mathrm{~d}, J=10.4 \mathrm{~Hz}, 1 \mathrm{H}), 3.43(\mathrm{~d}, J=10.2 \mathrm{~Hz}, 1 \mathrm{H}), 3.33(\mathrm{~d}, J=$ $10.4 \mathrm{~Hz}, 1 \mathrm{H}$ ), 3.00 (d, J=10.2 Hz, 1H), 2.46 (s, 3H), 0.86 (s, 3H), 0.52 (s, 3H);

${ }^{13} \mathrm{C} \mathrm{NMR}\left(151 \mathrm{MHz}, \mathrm{CDCl}_{3}\right) \delta 203.7,156.8,143.8,141.9,136.3,136.1,134.3,130.0$, 129.8, 129.1, 128.7, 127.2, 125.9 (q, $J=3.0 \mathrm{~Hz}$ ), 124.2, 123.8 (q, $J=272.0 \mathrm{~Hz}$ ), 59.3, $58.8,58.7,53.8,45.3,24.7,21.6$;

${ }^{19} \mathrm{~F} \mathrm{NMR}\left(376 \mathrm{MHz}, \mathrm{CDCl}_{3}\right) \delta-62.6$;

HRMS: (ESI) calcd for $\mathrm{C}_{28} \mathrm{H}_{26} \mathrm{~F}_{3} \mathrm{NO}_{3} \mathrm{SH}^{+}[\mathrm{M}+\mathrm{H}]^{+}$514.1658; found 514.1655.

The enantiomeric purity was established by HPLC analysis using a chiral column: OD$\mathrm{H}$ column, $30^{\circ} \mathrm{C}, n$-Hexane/i-Propanol $=90 / 10$ as eluent, $254 \mathrm{~nm}, 1 \mathrm{~mL} / \mathrm{min} . \mathrm{tR}=11$ $\min$ (major), $15 \mathrm{~min}$ (minor).

Optical Rotation: $[\alpha]_{D}^{28}-16.8$ (c 5.0, MeCN) for 99\% ee.

Absolute stereochemistry was determined through analogy with $\mathbf{3 a}$. 
<Chromatogram>

mAU

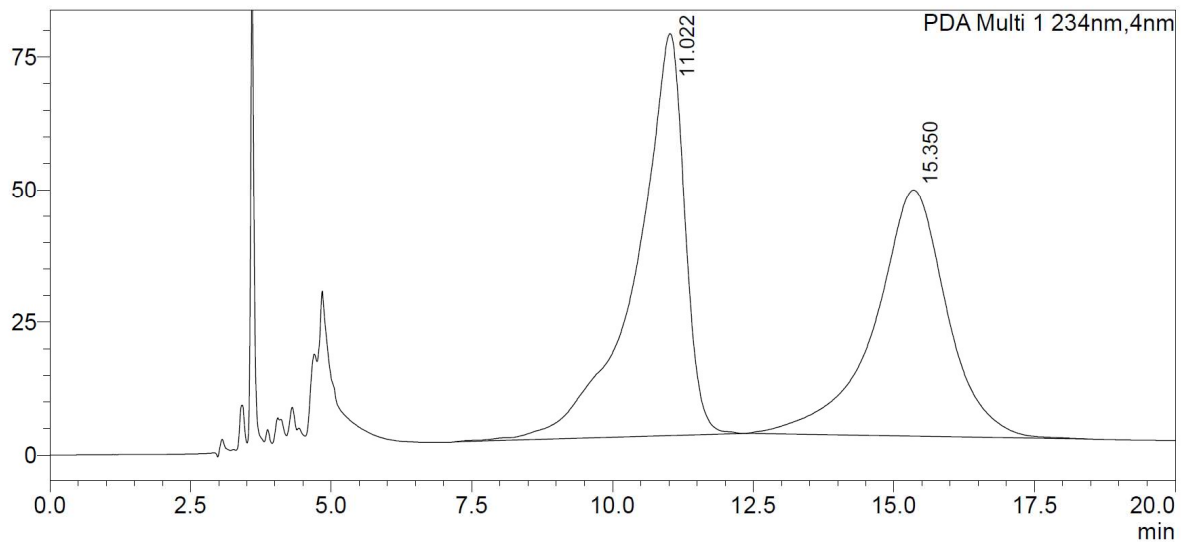

<Peak Table>

\begin{tabular}{|c|c|c|c|c|c|c|c|}
\hline & & & & & & \\
\hline \multicolumn{2}{|c|}{\begin{tabular}{|r|} 
Peak\# Ret. Time \\
1 \\
11.022
\end{tabular}} & 4544607 & 75745 & חמח 0 & Sim & M & Trame \\
\hline & & & & 0.000 & & IVI & \\
\hline & 15.350 & 4158044 & $403 / 9$ & 0.000 & & IVI & \\
\hline Total & & 8702651 & 122124 & & & & \\
\hline
\end{tabular}

\section{<Chromatogram>}

mAU

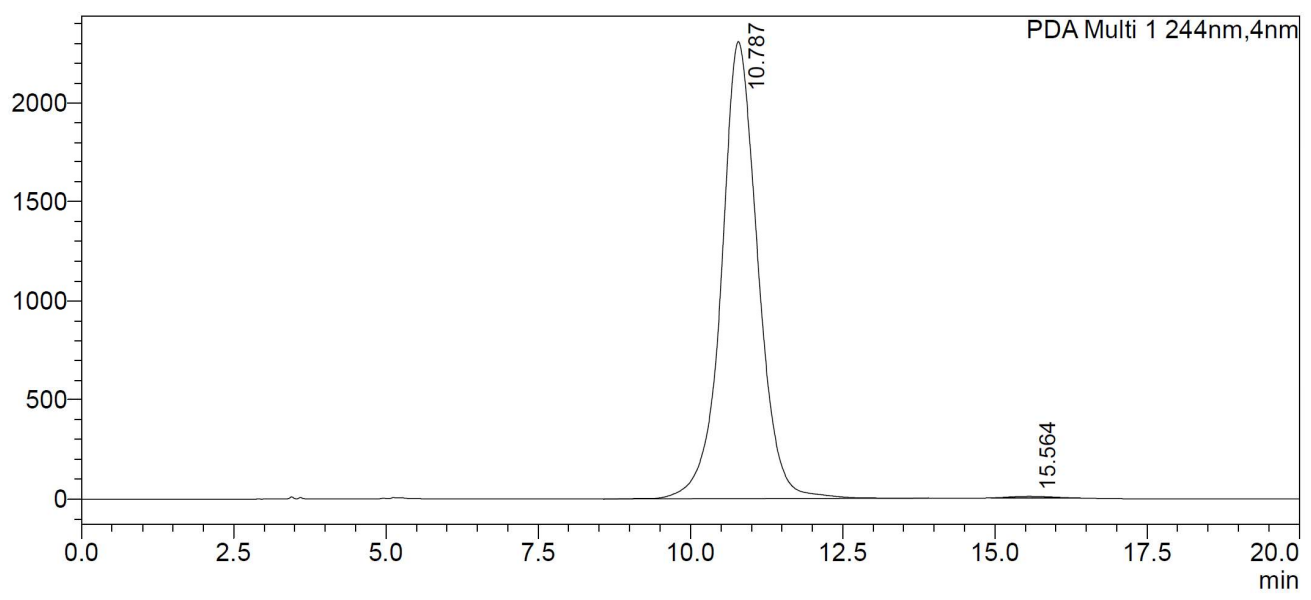

<Peak Table>

PDA Ch1 244nm

Peak\# Ret. Time

\begin{tabular}{|c|c|c|c|c|c|c|c|}
\hline Peak\# & Ret. Time & Area & Height & Conc. & Unit & Mark & Name \\
\hline 1 & 10.787 & 95590831 & 2306536 & 0.000 & & M & \\
\hline 2 & 15.564 & 382945 & 8018 & 0.000 & & M & \\
\hline Total & & 95973776 & 2314553 & & & & \\
\hline
\end{tabular}


ethyl 4-((1R,2S)-4',4'-dimethyl-3-oxo-1'-tosyl-2,3-dihydrospiro[indene-1,3'pyrrolidin]-2-yl)benzoate (3g)

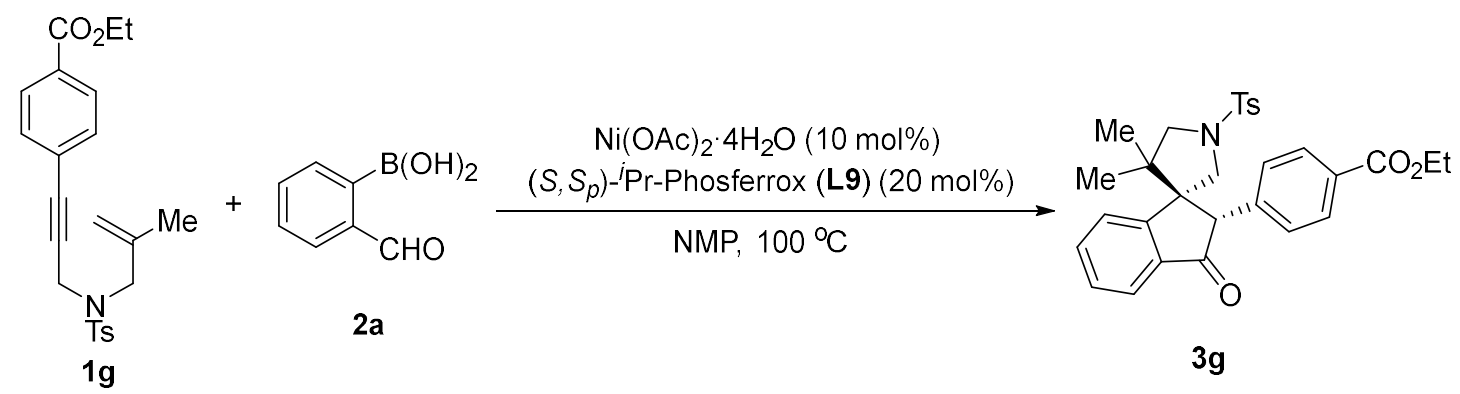

$\mathbf{3 g}$ was prepared according to general procedure using $\mathbf{1 g}(0.1 \mathrm{mmol}, 41.1 \mathrm{mg})$ and 2a $(0.3 \mathrm{mmol}, 45.0 \mathrm{mg})$ and was purified by silica gel column chromatography (petroleum ether/ethyl acetate $=10 / 1 \sim 5 / 1)$ to obtain $\mathbf{3 g}(39.3 \mathrm{mg}, 76 \%$ yield $)$.

${ }^{1} \mathrm{H} \mathrm{NMR}\left(400 \mathrm{MHz}, \mathrm{CDCl}_{3}\right)$ ठ 7.92-7.85 (m, 2H), 7.84-7.80 (m, 1H), 7.66-7.59 (m, 3H), 7.54-7.48 (m, 1H), $7.41(\mathrm{~d}, J=7.8 \mathrm{~Hz}, 1 \mathrm{H}), 7.33-7.28(\mathrm{~m}, 2 \mathrm{H}), 6.88-6.80(\mathrm{~m}, 2 \mathrm{H}), 4.37$ (q, $J=7.2 \mathrm{~Hz}, 2 \mathrm{H}), 3.83(\mathrm{~s}, 1 \mathrm{H}), 3.51(\mathrm{~d}, J=10.4 \mathrm{~Hz}, 1 \mathrm{H}), 3.39(\mathrm{~d}, J=10.3 \mathrm{~Hz}, 1 \mathrm{H})$, $3.31(\mathrm{~d}, J=10.4 \mathrm{~Hz}, 1 \mathrm{H}), 3.00$ (d, $J=10.3 \mathrm{~Hz}, 1 \mathrm{H}), 2.45$ (s, 3H), 1.38 (t, $J=7.1 \mathrm{~Hz}$, $3 \mathrm{H}), 0.84(\mathrm{~s}, 3 \mathrm{H}), 0.50(\mathrm{~s}, 3 \mathrm{H})$;

${ }^{13} \mathrm{C} \mathrm{NMR}\left(151 \mathrm{MHz}, \mathrm{CDCl}_{3}\right) \delta 203.9,166.0,156.9,143.7,142.9,136.4,136.0,134.2$, $130.1,129.8,129.8,129.0,128.3,127.1,125.9,124.0,61.0,59.3,58.8,58.7,53.8$, 45.1, 24.8, 21.5, 21.5, 14.3;

HRMS: (ESI) calcd for $\mathrm{C}_{30} \mathrm{H}_{32} \mathrm{NO}_{5} \mathrm{~S}^{+}[\mathrm{M}+\mathrm{H}]^{+}$518.1996; found 518.1998.

The enantiomeric purity was established by HPLC analysis using a chiral column: OD$\mathrm{H}$ column, $30^{\circ} \mathrm{C}, n$-Hexane/i-Propanol $=90 / 10$ as eluent, $254 \mathrm{~nm}, 1 \mathrm{~mL} / \mathrm{min}$. $\mathrm{tR}=17$ $\min$ (major), 22 min (minor).

Optical Rotation: $[\alpha]_{D}^{28}-112.3$ (c 5.0, MeCN) for $94 \%$ ee.

Absolute stereochemistry was determined through analogy with $\mathbf{3 a}$. 


\section{<Chromatogram>}

mAU

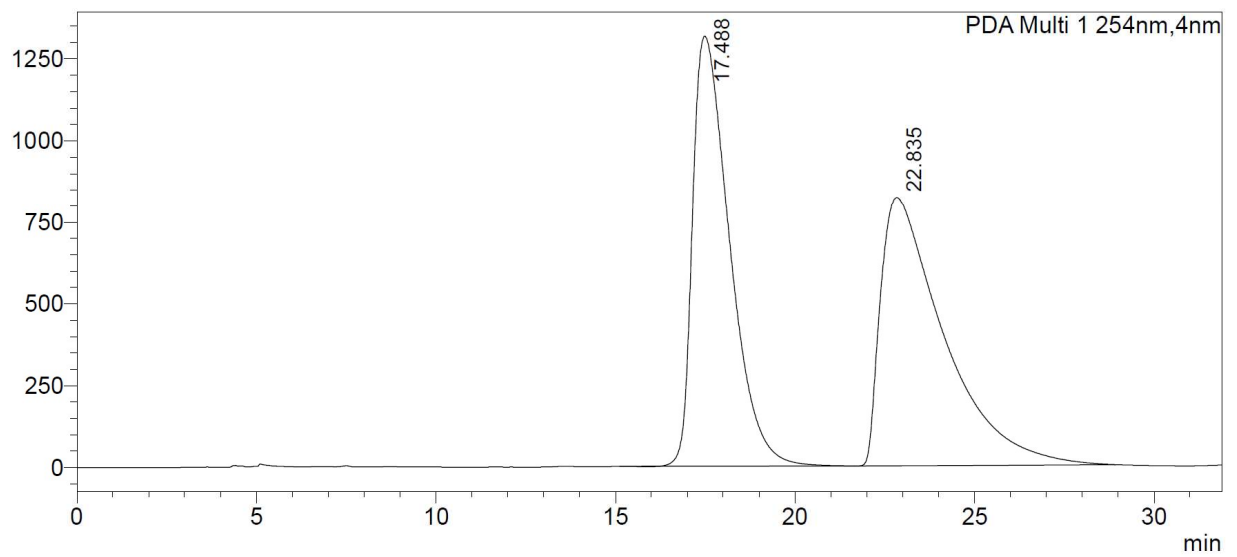

<Peak Table>

PDA Ch1 254nm

\begin{tabular}{|r|r|r|r|r|r|r|r|}
\hline Peak\# Ret. Time & \multicolumn{1}{|c|}{ Area } & Height & Conc. & Unit & Mark & Name \\
\hline 1 & 17.488 & 94275244 & 1314798 & 0.000 & & $\mathrm{M}$ & \\
\hline 2 & 22.835 & 100610033 & 821678 & 0.000 & & $\mathrm{M}$ & \\
\hline Total & 194885277 & 2136476 & & & & \\
\hline
\end{tabular}

\section{<Chromatogram>}

mAU

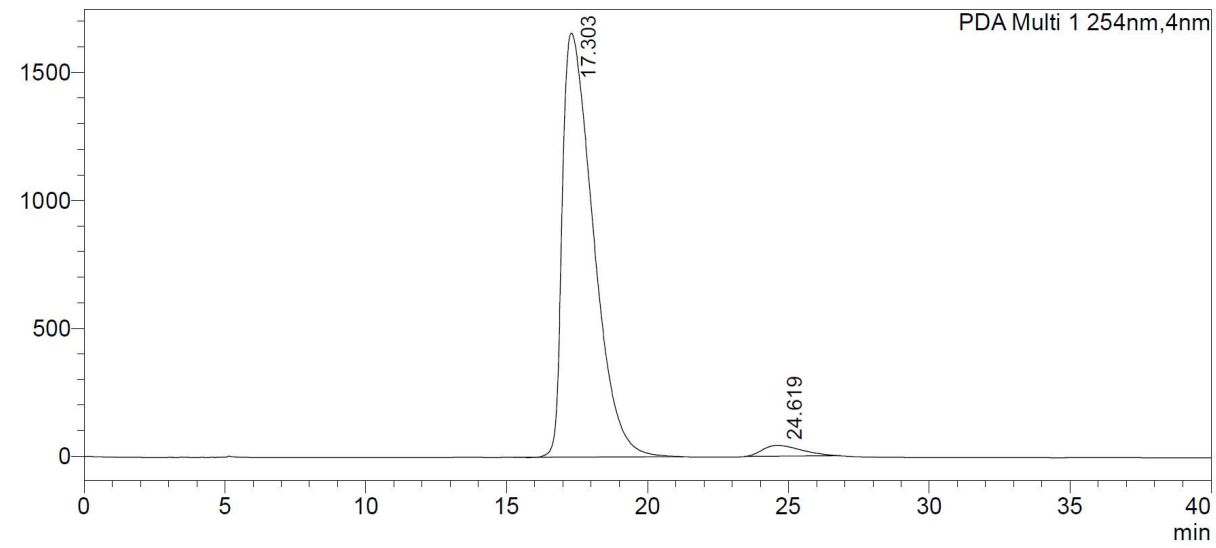

<Peak Table>

PDA Ch1 254nm

\begin{tabular}{|r|r|r|r|r|r|r|r|}
\hline Peak\# Ret. Time & \multicolumn{1}{|c|}{ Area } & Height & Conc. & Unit & Mark & Name \\
\hline 1 & 17.303 & 126377068 & 1656960 & 0.000 & & $\mathrm{M}$ & \\
\hline 2 & 24.619 & 4135689 & 42778 & 0.000 & & $\mathrm{M}$ & \\
\hline Total & 130512757 & 1699738 & & & & \\
\hline
\end{tabular}


(1R,2S)-4',4'-dimethyl-2-(4-(4,4,5,5-tetramethyl-1,3,2-dioxaborolan-2-yl)phenyl)1'-tosylspiro[indene-1,3'-pyrrolidin]-3(2H)-one (3h)

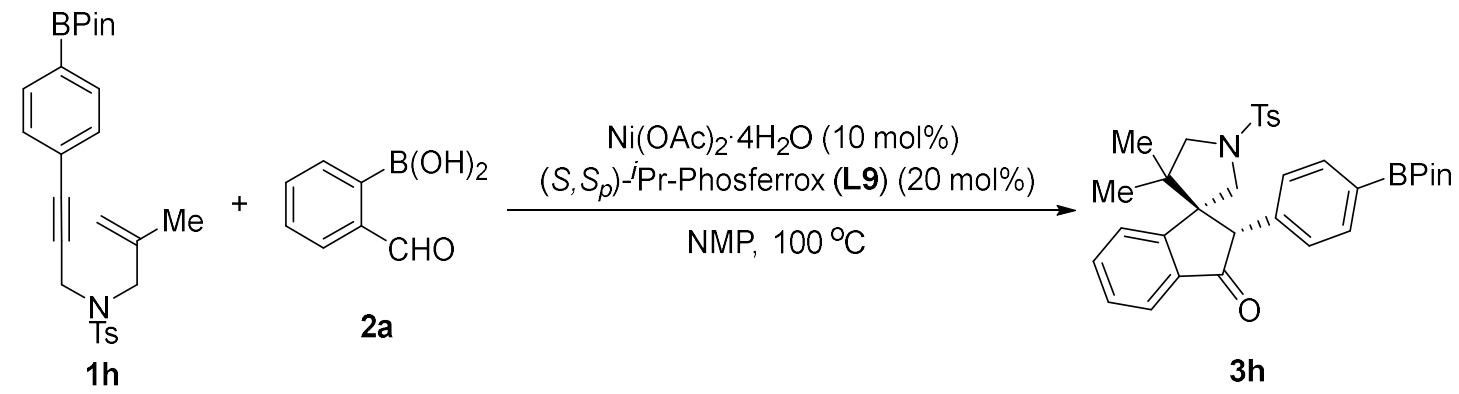

3h was prepared according to general procedure using $1 \mathrm{~h}(0.1 \mathrm{mmol}, 46.6 \mathrm{mg})$ and 2a $(0.3 \mathrm{mmol}, 45.0 \mathrm{mg})$ and was purified by silica gel column chromatography (petroleum ether/ethyl acetate $=10 / 1 \sim 5 / 1)$ to obtain $3 \mathrm{~h}(24.6 \mathrm{mg}, 43 \%$ yield $)$.

${ }^{1} \mathrm{H} \mathrm{NMR}\left(400 \mathrm{MHz}, \mathrm{CDCl}_{3}\right)$ ס 7.86-7.79 (m, 1H), 7.69-7.57 (m, 5H), 7.53-7.47 (m, 1H), $7.39(\mathrm{~d}, J=7.8 \mathrm{~Hz}, 1 \mathrm{H}), 7.31(\mathrm{~d}, J=8.0 \mathrm{~Hz}, 2 \mathrm{H}), 6.76(\mathrm{~d}, J=7.6 \mathrm{~Hz}, 2 \mathrm{H}), 3.78(\mathrm{~s}, 1 \mathrm{H})$, 3.50 (d, J = $10.4 \mathrm{~Hz}, 1 \mathrm{H}$ ), 3.38 (d, J = 10.5 Hz, 1H), 3.29 (d, J=10.4 Hz, 1H), 3.05 (d, $J=10.4 \mathrm{~Hz}, 1 \mathrm{H}), 2.47$ (s, 3H), $1.34(\mathrm{~s}, 12 \mathrm{H}), 0.84(\mathrm{~s}, 3 \mathrm{H}), 0.48$ (s, 3H);

${ }^{13} \mathrm{C}$ NMR $\left(151 \mathrm{MHz}, \mathrm{CDCl}_{3}\right) \delta$ 204.5, 157.3, 143.6, 141.0, 136.6, 135.8, 135.4, 134.4, $129.8,128.8,127.6,127.1,125.9,123.9,83.9,59.4,58.9,58.8,54.1$, 45.0, 25.0, 24.9, 24.8, 21.6, 21.4;

HRMS: (ESI) calcd for $\mathrm{C}_{33} \mathrm{H}_{39} \mathrm{NBO}_{5} \mathrm{~S}^{+}[\mathrm{M}+\mathrm{H}]^{+} 572.2637$; found 572.2623 .

The enantiomeric purity was established by HPLC analysis using a chiral column: OD$\mathrm{H}$ column, $30^{\circ} \mathrm{C}, n$-Hexane/ $i$-Propanol $=95 / 5$ as eluent, $254 \mathrm{~nm}, 1 \mathrm{~mL} / \mathrm{min} . \mathrm{tR}=15 \mathrm{~min}$ (major), $21 \mathrm{~min}$ (minor).

Optical Rotation: $[\alpha]_{D}^{28}-79.4$ (c 5.0, MeCN) for $91 \%$ ee.

Absolute stereochemistry was determined through analogy with $\mathbf{3 a}$. 
<Chromatogram>

mAU

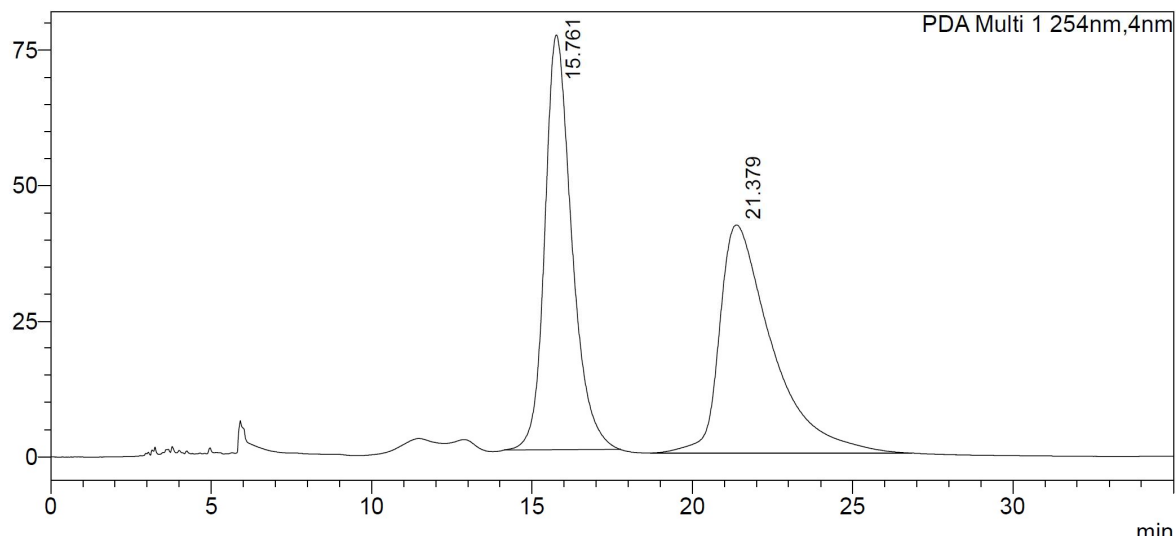

<Peak Table>

\begin{tabular}{|c|c|c|c|c|c|c|c|}
\hline \multirow{2}{*}{\multicolumn{2}{|c|}{$\begin{array}{l}\text { PDA Ch1 254nm } \\
\text { Peak\# Ret. Time }\end{array}$}} & & & & & & \\
\hline & & Area & Height & Conc. & Unit & Mark & Name \\
\hline 1 & 15.761 & 4478120 & 76459 & 0.000 & & $\mathrm{M}$ & \\
\hline 2 & 21.379 & 4788843 & 42080 & 0.000 & & $M$ & \\
\hline Total & & 9266963 & 118539 & & & & \\
\hline
\end{tabular}

\section{$<$ Chromatogram $>$}

mAU

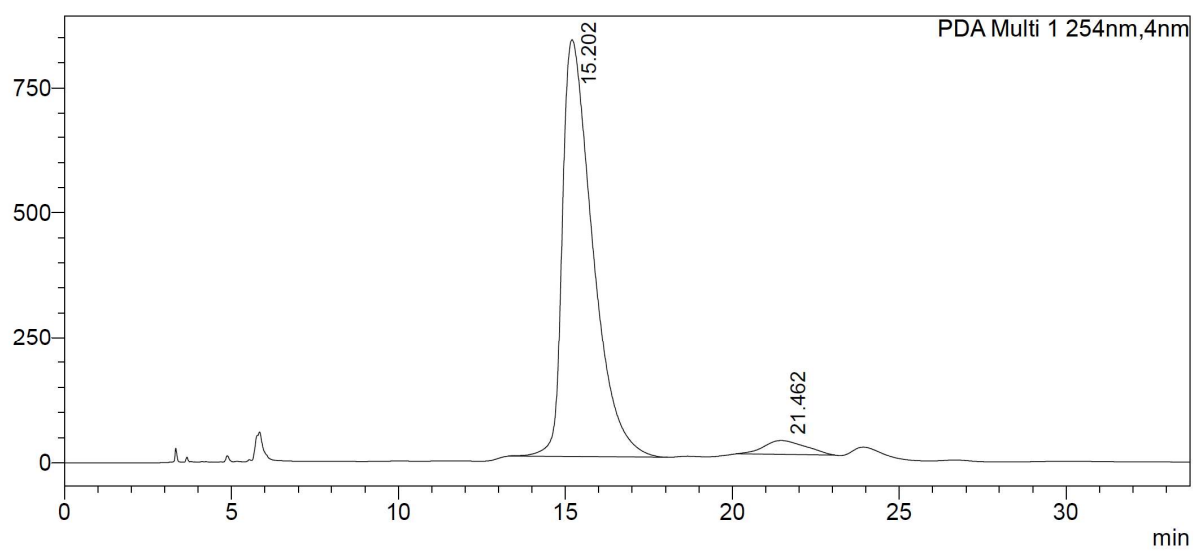

<Peak Table>

PDA Ch1 254nm

\begin{tabular}{|r|r|r|r|r|r|r|r|}
\hline Peak\# Ret. Time & \multicolumn{1}{|c|}{ Area } & Height & Conc. & Unit & Mark & Name \\
\hline 1 & 15.202 & 52520227 & 833697 & 0.000 & & M & \\
\hline 2 & 21.462 & 2392629 & 27651 & 0.000 & & $M$ & \\
\hline Total & & 54912856 & 861348 & & & & \\
\hline
\end{tabular}


(1R,2S)-2-(dibenzo[b,c]furan-3-yl)-4',4'-dimethyl-1'-tosylspiro[indene-1,3'pyrrolidin]-3(2H)-one (3i)<smiles>C=C(C)CN(S)CC#Cc1ccc2c(c1)oc1ccccc12</smiles>

$1 \mathrm{i}$

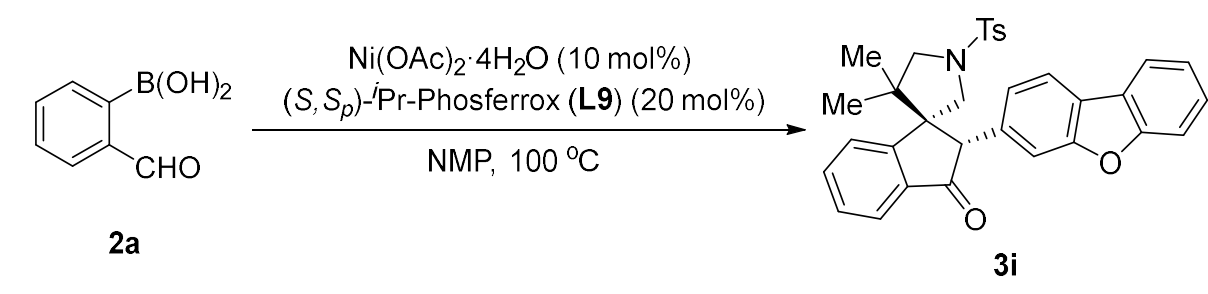

$\mathbf{3 i}$ was prepared according to general procedure using $\mathbf{1 i}(0.1 \mathrm{mmol}, 42.9 \mathrm{mg})$ and $\mathbf{2 a}$ $(0.3 \mathrm{mmol}, 45.0 \mathrm{mg})$ and was purified by silica gel column chromatography (petroleum ether/ethyl acetate $=10 / 1 \sim 5 / 1)$ to obtain $3 \mathbf{i}(48.7 \mathrm{mg}, 91 \%$ yield $)$.

${ }^{1} \mathrm{H}$ NMR $\left(400 \mathrm{MHz}, \mathrm{CDCl}_{3}\right)$ ठ 7.90-7.86 (m, 1H), 7.85-7.80 (m, 1H), 7.69-7.64 (m, 1H), 7.59-7.52 (m, 4H), 7.51-7.45 (m, 2H), 7.43-7.38 (m, 2H), 7.36-7.30 (m, 1H), 7.23-7.17 (m, 2H), 6.84-6.76 (m, 1H), $3.95(\mathrm{~s}, 1 \mathrm{H}), 3.56(\mathrm{~d}, J=10.5 \mathrm{~Hz}, 1 \mathrm{H}), 3.45(\mathrm{~d}, J=10.4$ $\mathrm{Hz}, 1 \mathrm{H}$ ), 3.34 (d, J=10.5 Hz, 1H), 3.09 (d, J = 10.3 Hz, 1H), 2.39 (s, 3H), $0.91(\mathrm{~s}, 3 \mathrm{H})$, $0.55(\mathrm{~s}, 3 \mathrm{H})$;

${ }^{13} \mathrm{C}$ NMR $\left(151 \mathrm{MHz}, \mathrm{CDCl}_{3}\right) \delta 204.9,157.2,156.5,155.4,143.6,136.5,135.9,134.3$, $132.5,129.6,128.9,127.6,127.1,126.0,124.8,124.1,123.7,122.8,120.8,112.1$, $111.8,59.5,59.0,58.56,54.10,45.1,25.0,21.5,21.5$

HRMS: (ESI) calcd for $\mathrm{C}_{33} \mathrm{H}_{30} \mathrm{NO}_{4} \mathrm{~S}^{+}[\mathrm{M}+\mathrm{H}]^{+} 536.1890$; found 536.1881.

The enantiomeric purity was established by HPLC analysis using a chiral column: OD$\mathrm{H}$ column, $30^{\circ} \mathrm{C}, n$-Hexane/i-Propanol $=80 / 20$ as eluent, $254 \mathrm{~nm}, 1 \mathrm{~mL} / \mathrm{min}$. $\mathrm{tR}=10$ $\min$ (major), 13 min (minor).

Optical Rotation: $[\alpha]_{D}^{28}-81.8$ (c 5.0, MeCN) for $95 \%$ ee.

Absolute stereochemistry was determined through analogy with $\mathbf{3 a}$. 


\section{$<$ Chromatogram >}

mAU

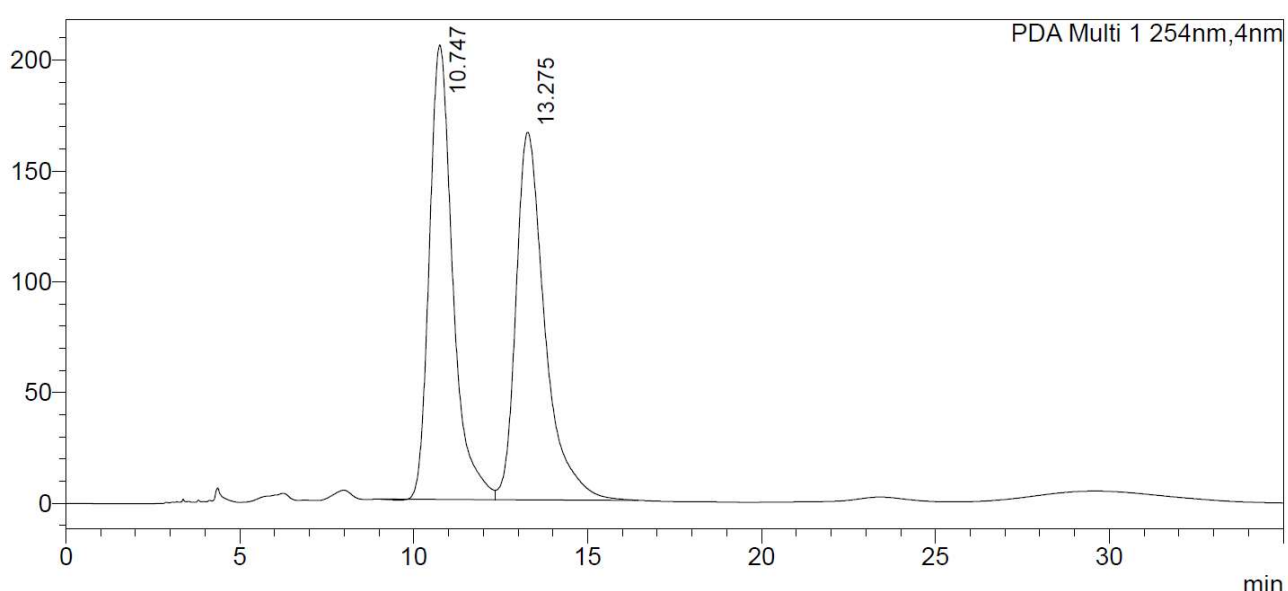

<Peak Table>

PDA Ch1 254nm

\begin{tabular}{|r|r|r|r|r|r|r|r|}
\hline Peak\# Ret. Time & \multicolumn{1}{|c|}{ Area } & Height & Conc. & Unit & Mark & Name \\
\hline 1 & 10.747 & 9598453 & 204919 & 0.000 & & $\mathrm{M}$ & \\
\hline 2 & 13.275 & 9569950 & 165762 & 0.000 & & $\mathrm{~V} \mathrm{M}$ & \\
\hline Total & & 19168403 & 370681 & & & & \\
\hline
\end{tabular}

\section{<Chromatogram>}

mAU

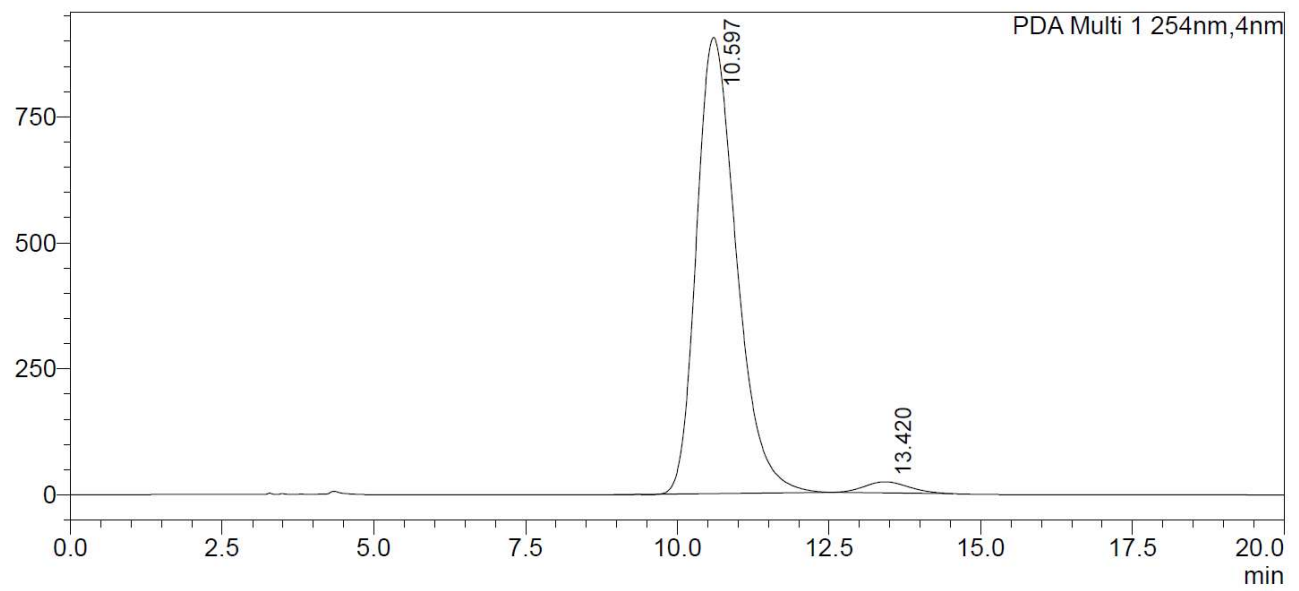

<Peak Table>

PDA Ch1 254nm

Peak\# Ret. Time

$1 \quad 10.597$

Area

Height $\quad$ Conc.

\begin{tabular}{|l|l|l|}
\hline 0.000 & Unit & M \\
\hline 0.000 & & $M$ \\
\hline
\end{tabular}

Name

\begin{tabular}{r|r|r|r|}
\hline 2 & 13.420 & 1108959 & 21941 \\
\hline
\end{tabular}

\begin{tabular}{|l|r|r|}
\hline Total & 42639337 & 927250 \\
\hline
\end{tabular} 
(1R,2S)-4',4'-dimethyl-2-(9-phenyl-9H-carbazol-2-yl)-1'-tosylspiro[indene-1,3'pyrrolidin]-3(2H)-one (3j)

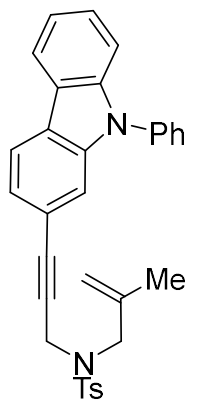

$1 \mathrm{j}$

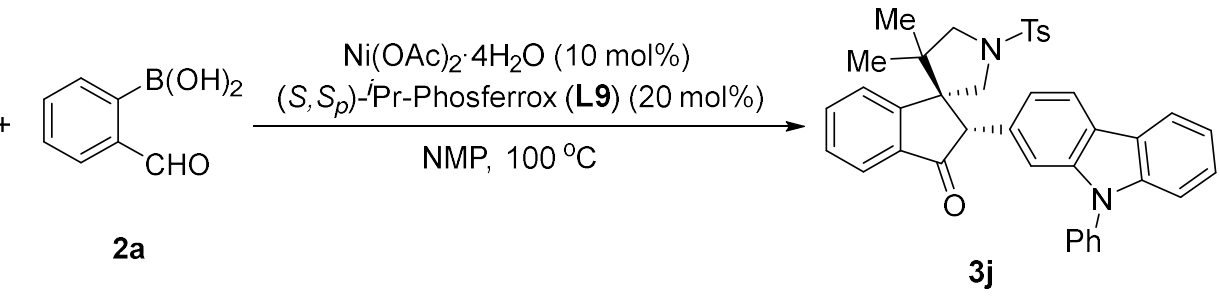

3j

3j was prepared according to general procedure using $1 \mathbf{j}(0.1 \mathrm{mmol}, 50.4 \mathrm{mg})$ and $\mathbf{2 a}$ $(0.3 \mathrm{mmol}, 45.0 \mathrm{mg})$ and was purified by silica gel column chromatography (petroleum ether/ethyl acetate $=10 / 1 \sim 5 / 1)$ to obtain $3 \mathbf{j}(54.3 \mathrm{mg}, 89 \%$ yield $)$.

${ }^{1} \mathrm{H}$ NMR $\left(600 \mathrm{MHz}, \mathrm{CDCl}_{3}\right) \delta 8.00(\mathrm{~d}, J=7.8 \mathrm{~Hz}, 1 \mathrm{H}), 7.87(\mathrm{~d}, J=7.4 \mathrm{~Hz}, 1 \mathrm{H}), 7.66-$ $7.57(\mathrm{~m}, 6 \mathrm{H})$, 7.56-7.50 (m, 3H), 7.48-7.39 (m, 4H), 7.28-7.26 (m, 1H), 7.23-7.15 (m, 3H), 6.74-6.67 (m, 1H), 3.99 (s, 1H), 3.55 (d, J = $10.4 \mathrm{~Hz}, 1 \mathrm{H}), 3.48$ (d, J = $10.4 \mathrm{~Hz}$, 1H), $3.34(\mathrm{~d}, J=10.4 \mathrm{~Hz}, 1 \mathrm{H}), 3.17(\mathrm{~d}, J=10.5 \mathrm{~Hz}, 1 \mathrm{H}), 2.37$ (s, 3H), $0.94(\mathrm{~s}, 3 \mathrm{H})$, $0.54(\mathrm{~s}, 3 \mathrm{H})$;

${ }^{13} \mathrm{C}$ NMR $\left(151 \mathrm{MHz}, \mathrm{CDCl}_{3}\right) \delta 205.4,157.4,143.5,141.2,140.1,137.4,136.7,135.7$, $134.4,129.9,129.7,129.4,128.8,127.6,127.1,127.0,126.3,126.0,124.0,123.7$, $122.9,120.3,120.0,110.3,109.9,59.5,59.1,58.9,54.3,45.1,25.0,21.5,21.5$;

HRMS: (ESI) calcd for $\mathrm{C}_{39} \mathrm{H}_{35} \mathrm{~N}_{2} \mathrm{O}_{3} \mathrm{~S}^{+}[\mathrm{M}+\mathrm{H}]^{+}$611.2363; found 611.2378.

The enantiomeric purity was established by HPLC analysis using a chiral column: AD$\mathrm{H}$ column, $30^{\circ} \mathrm{C}, n$-Hexane/i-Propanol $=70 / 30$ as eluent, $254 \mathrm{~nm}, 1 \mathrm{~mL} / \mathrm{min}$. $\mathrm{tR}=12$ $\min$ (major), 21 min (minor).

Optical Rotation: $[\alpha]_{D}^{28}-42.9$ (c 5.0, MeCN) for $99 \%$ ee. Absolute stereochemistry was determined through analogy with $\mathbf{3 a}$. 
mAU

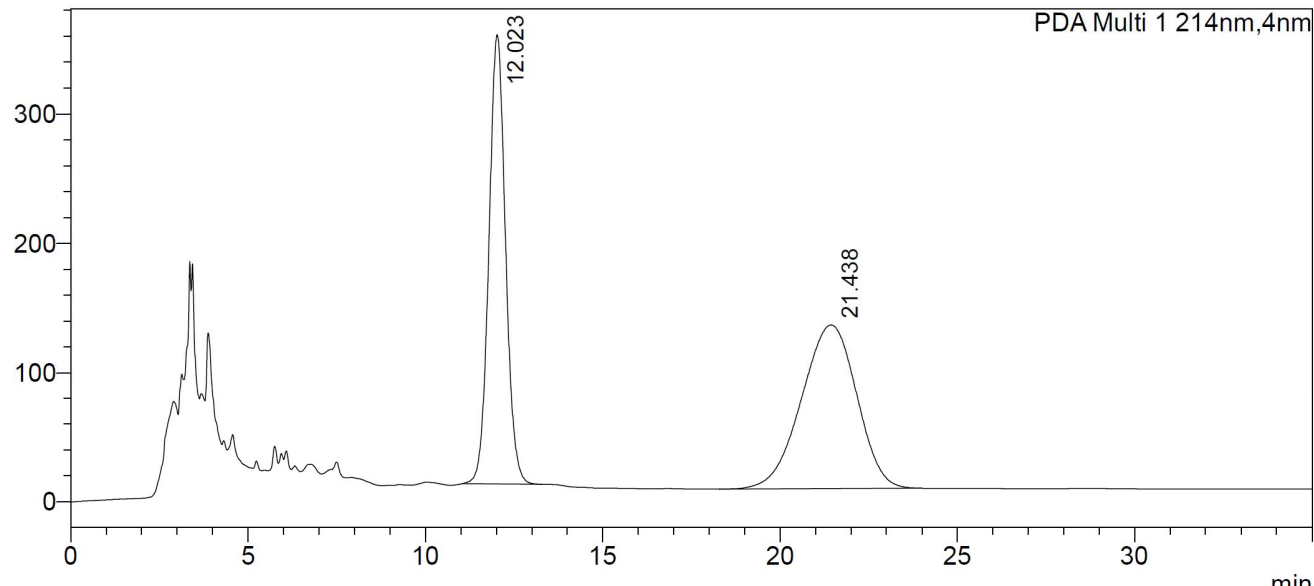

<Peak Table>

PDA Ch1 214nm

Peak\# Ret. Time

\begin{tabular}{|c|c|c|c|c|c|c|c|}
\hline Peak\# & t. Time & Area & Height & Conc. & Unit & Mark & Name \\
\hline 1 & 12.023 & 11354681 & 347493 & 45.235 & & $M$ & \\
\hline 2 & 21.438 & 13746588 & 127052 & 54.765 & & $\mathrm{M}$ & \\
\hline Total & & 25101269 & 474545 & & & & \\
\hline
\end{tabular}

mAU

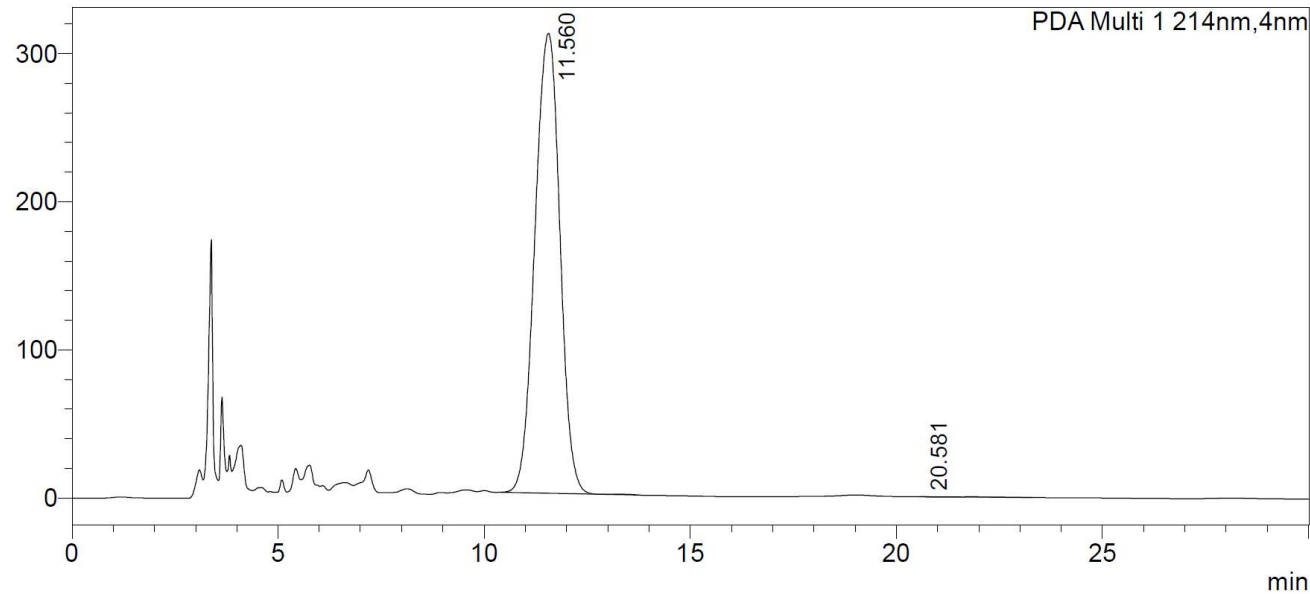

<Peak Table>

PDA Ch1 214nm

$$
\text { Peak\# Ret. Time }
$$

Height

Conc.

Unit Mark

Name

\begin{tabular}{|r|r|r|r|r|r|r|r|}
\hline 1 & 11.560 & 13086970 & 310585 & 0.000 & $\mathrm{M}$ & \\
\hline 2 & 20.581 & 8896 & 1 & 0.000 & $\mathrm{M}$ & \\
\hline Total & & 13095866 & 310586 & & & & \\
\hline
\end{tabular}


(1R,2S)-4',4'-dimethyl-2-((8R,9S,13S,14S)-13-methyl-17-oxo-

7,8,9,11,12,13,14,15,16,17-decahydro-6H-cyclopenta[a]phenanthren-3-yl)-1'tosylspiro[indene-1,3'-pyrrolidin]-3(2H)-one (3k)
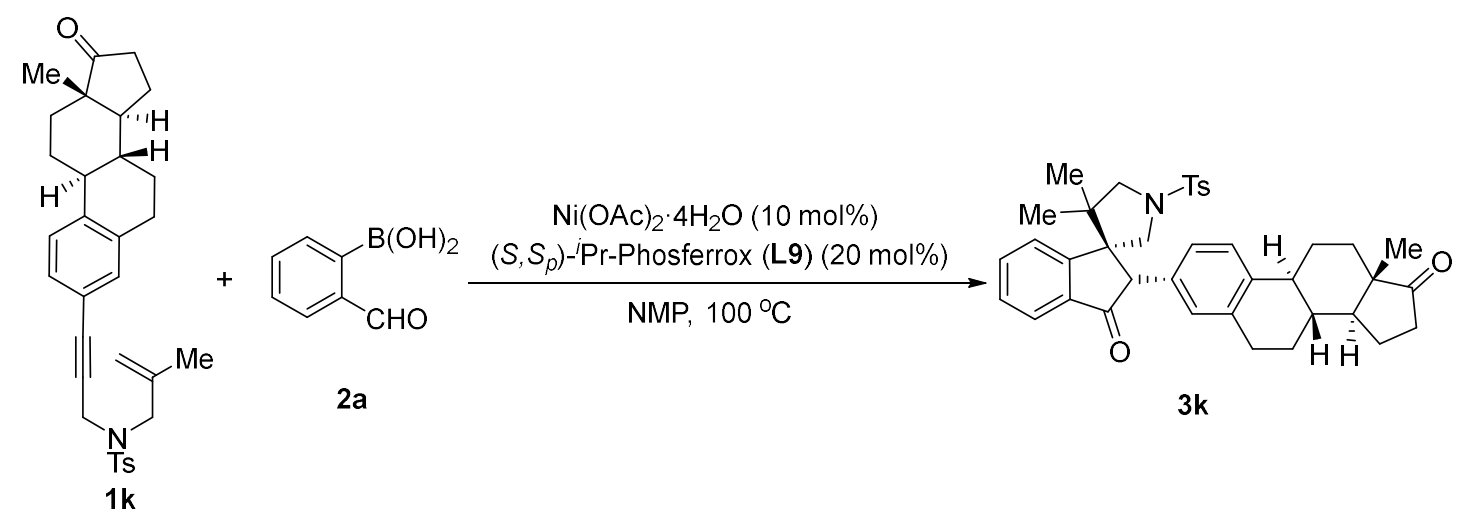

3k was prepared according to general procedure using $1 \mathbf{a}(0.1 \mathrm{mmol}, 51.5 \mathrm{mg})$ and $2 \mathrm{a}$ $(0.3 \mathrm{mmol}, 45.0 \mathrm{mg})$ and was purified by silica gel column chromatography (petroleum ether/ethyl acetate $=10 / 1 \sim 5 / 1)$ to obtain $3 \mathbf{k}(57.8 \mathrm{mg}, 93 \%$ yield $)$.

${ }^{1} \mathrm{H}$ NMR $\left(600 \mathrm{MHz}, \mathrm{CDCl}_{3}\right) \delta 7.80(\mathrm{~d}, J=7.2 \mathrm{~Hz}, 1 \mathrm{H}), 7.70-7.65(\mathrm{~m}, 2 \mathrm{H}), 7.54$ (td, $J=$ 7.5, 1.2 Hz, 1H), 7.50-7.44 (m, 1H), $7.34(\mathrm{~d}, J=7.9 \mathrm{~Hz}, 2 \mathrm{H}), 7.23(\mathrm{~d}, J=7.8 \mathrm{~Hz}, 1 \mathrm{H})$, 7.09 (d, J=7.9 Hz, 1H), 6.54-6.46 (m, 2H), $3.72(\mathrm{~s}, 1 \mathrm{H}), 3.44$ (d, J=10.4 Hz, 1H), 3.38 (d, $J=10.5 \mathrm{~Hz}, 1 \mathrm{H}), 3.30$ (d, $J=10.4 \mathrm{~Hz}, 1 \mathrm{H}), 3.19$ (d, $J=10.5 \mathrm{~Hz}, 1 \mathrm{H}), 2.81-2.74(\mathrm{~m}$, 2H), 2.54-2.45 (m, 4H), 2.37-2.31 (m, 1H), 2.28-2.22 (m, 1H), 2.18-2.10 (m, 1H), 2.08$2.02(\mathrm{~m}, 1 \mathrm{H}), 2.01-1.92(\mathrm{~m}, 2 \mathrm{H}), 1.65-1.56(\mathrm{~m}, 2 \mathrm{H}), 1.55-1.38(\mathrm{~m}, 3 \mathrm{H}), 0.92(\mathrm{~s}, 3 \mathrm{H})$, $0.89(\mathrm{~s}, 3 \mathrm{H}), 0.42(\mathrm{~s}, 3 \mathrm{H})$;

${ }^{13} \mathrm{C} \mathrm{NMR}\left(151 \mathrm{MHz}, \mathrm{CDCl}_{3}\right) \delta 220.9,205.2,157.5,143.6,139.2,137.1,136.6,135.6$, 135.2, 134.5, 129.7, 128.7, 127.2, 125.9, 125.7, 125.4, 123.8, 59.3, 58.7, 58.2, 54.3, $50.4,47.9,44.9,44.2,37.8,35.8,31.5,29.3,26.3,25.4,25.1,21.6,21.5,21.2,13.8$; HRMS: (ESI) calcd for $\mathrm{C}_{39} \mathrm{H}_{44} \mathrm{NO}_{4} \mathrm{~S}^{+}[\mathrm{M}+\mathrm{H}]^{+}$622.2986; found 622.2971.

Optical Rotation: $[\alpha]_{D}^{28}-35.2$ (c 5.0, MeCN) for $>20 / 1$ d.r. Absolute stereochemistry was determined through analogy with $\mathbf{3 a}$. 
$(1 R, 2 S)-2,4$ ',4'-trimethyl-1'-tosylspiro[indene-1,3'-pyrrolidin]-3(2H)-one (3I)

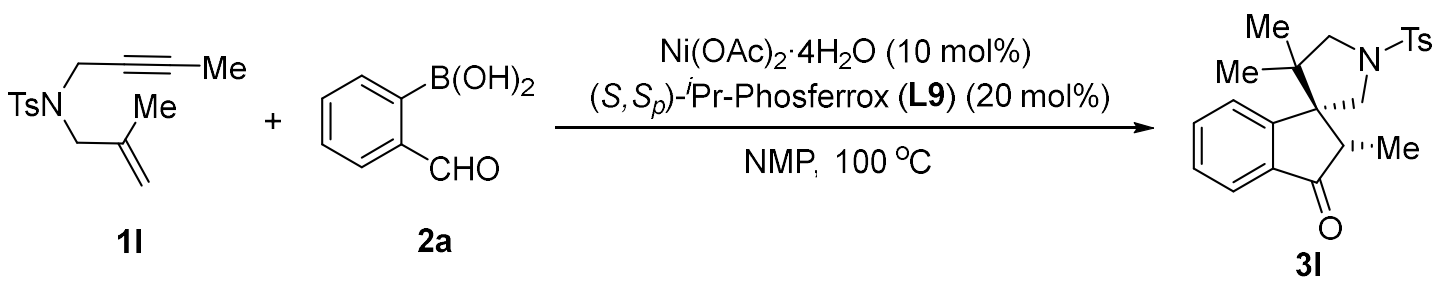

3I was prepared according to general procedure using $11(0.1 \mathrm{mmol}, 27.7 \mathrm{mg})$ and $2 \mathrm{a}$ $(0.3 \mathrm{mmol}, 45.0 \mathrm{mg})$ and was purified by silica gel column chromatography (petroleum ether/ethyl acetate $=10 / 1 \sim 5 / 1)$ to obtain $3 \mathbf{I}(16.1 \mathrm{mg}, 42 \%$ yield $)$.

${ }^{1} \mathrm{H}$ NMR $\left(600 \mathrm{MHz}, \mathrm{CDCl}_{3}\right)$ ס 7.84-7.76 (m, 2H), 7.74-7.70 (m, 1H), 7.56-7.49 (m, 1H), 7.45-7.32 (m, 4H), $3.62(\mathrm{~d}, J=9.8 \mathrm{~Hz}, 1 \mathrm{H}), 3.54(\mathrm{~d}, J=9.8 \mathrm{~Hz}, 1 \mathrm{H}), 3.50(\mathrm{~d}, J=10.3$ $\mathrm{Hz}, 1 \mathrm{H}$ ), $3.31(\mathrm{~d}, J=10.3 \mathrm{~Hz}, 1 \mathrm{H}), 2.59$ (q, $J=7.6 \mathrm{~Hz}, 1 \mathrm{H}), 2.47(\mathrm{~s}, 3 \mathrm{H}), 1.12(\mathrm{~d}, J=$ $7.6 \mathrm{~Hz}, 3 \mathrm{H}), 0.72(\mathrm{~s}, 3 \mathrm{H}), 0.45(\mathrm{~s}, 3 \mathrm{H})$;

${ }^{13} \mathrm{C}$ NMR $\left(151 \mathrm{MHz}, \mathrm{CDCl}_{3}\right) \delta 207.5,156.0,143.8,135.4,134.8,134.3,129.83,128.5$, $127.3,125.9,123.8,59.4,57.5,52.6,46.4,44.6,24.5,21.6,21.3,15.1$;

HRMS: (ESI) calcd for $\mathrm{C}_{22} \mathrm{H}_{26} \mathrm{NO}_{3} \mathrm{~S}^{+}[\mathrm{M}+\mathrm{H}]^{+} 384.1628$; found 384.1632.

The enantiomeric purity was established by HPLC analysis using a chiral column: OD$\mathrm{H}$ column, $30^{\circ} \mathrm{C}, n$-Hexane/i-Propanol $=90 / 10$ as eluent, $254 \mathrm{~nm}, 1 \mathrm{~mL} / \mathrm{min} . \mathrm{tR}=12$ $\min$ (major), 14 min (minor).

Optical Rotation: $[\alpha]_{D}^{28}-56.6$ (c 5.0, MeCN) for $90 \%$ ee.

Absolute stereochemistry was determined through analogy with $\mathbf{3 a}$. 
<Chromatogram>

mAU

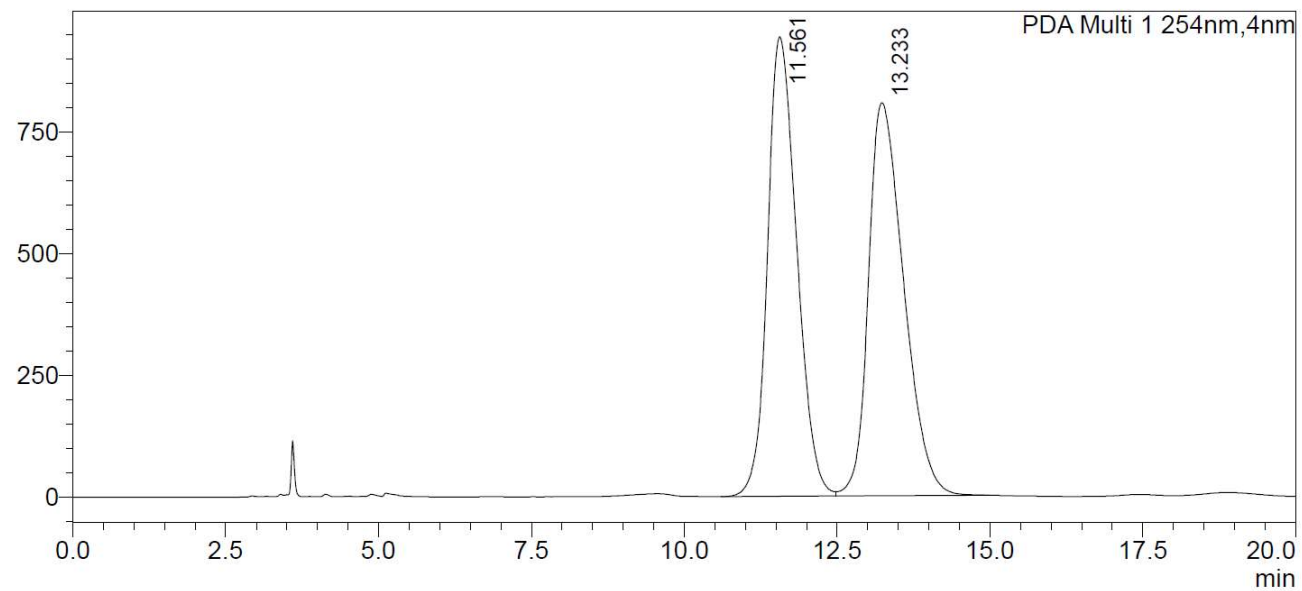

<Peak Table>

PDA Ch1 254nm

\begin{tabular}{|r|r|r|r|r|r|r|r|}
\hline Peak\# Ret. Time & Area & Height & Conc. & Unit & Mark & Name \\
\hline 1 & 11.561 & 31386864 & 944573 & 0.000 & & $\mathrm{M}$ & \\
\hline 2 & 13.233 & 32402004 & 807671 & 0.000 & & V M & \\
\hline Total & & 63788867 & 1752245 & & & &
\end{tabular}

<Chromatogram>

mAU

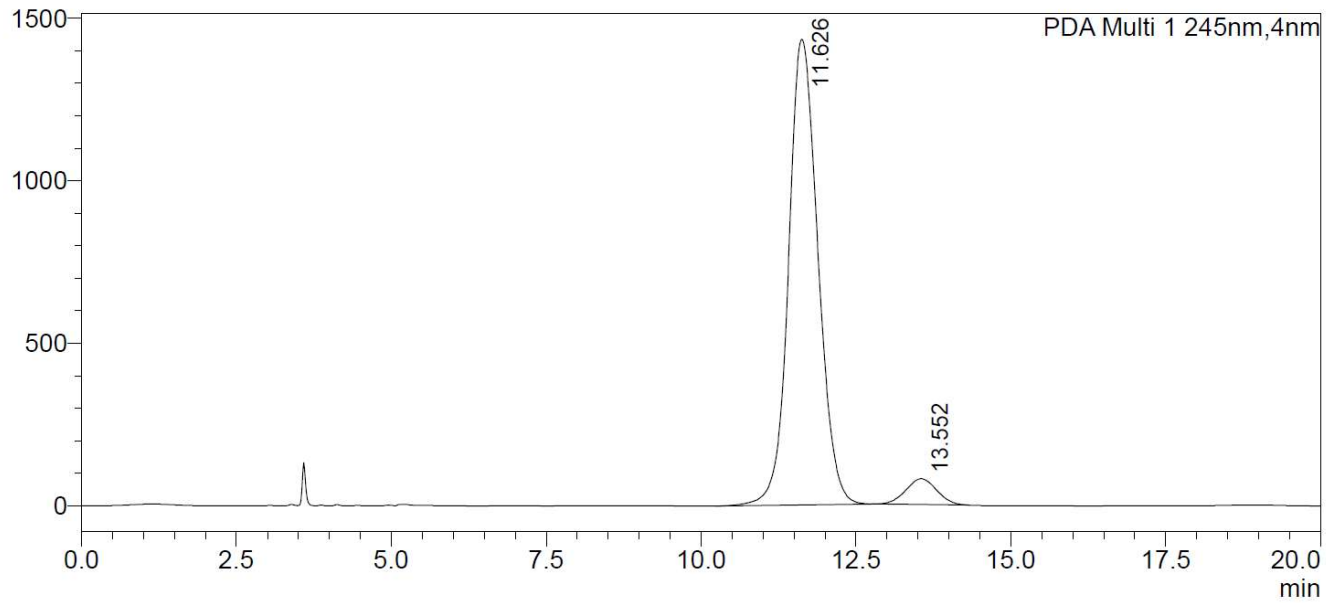

<Peak Table>

PDA Ch1 245nm

\begin{tabular}{|r|r|r|r|r|r|r|r|}
\hline Peak\# Ret. Time & \multicolumn{1}{|c|}{ Area } & \multicolumn{1}{c|}{ Height } & Conc. & Unit & Mark & Name \\
\hline 1 & 11.626 & 47141205 & 1432488 & 94.497 & & $\mathrm{M}$ & \\
\hline 2 & 13.552 & 2745270 & 79157 & 5.503 & & $\mathrm{M}$ & \\
\hline Total & & 49886474 & 1511645 & & & &
\end{tabular}


(1R,2S)-4',4'-dimethyl-2-methylene-1'-tosylspiro[indene-1,3'-pyrrolidin]-3(2H)one $(3 \mathrm{~m})$

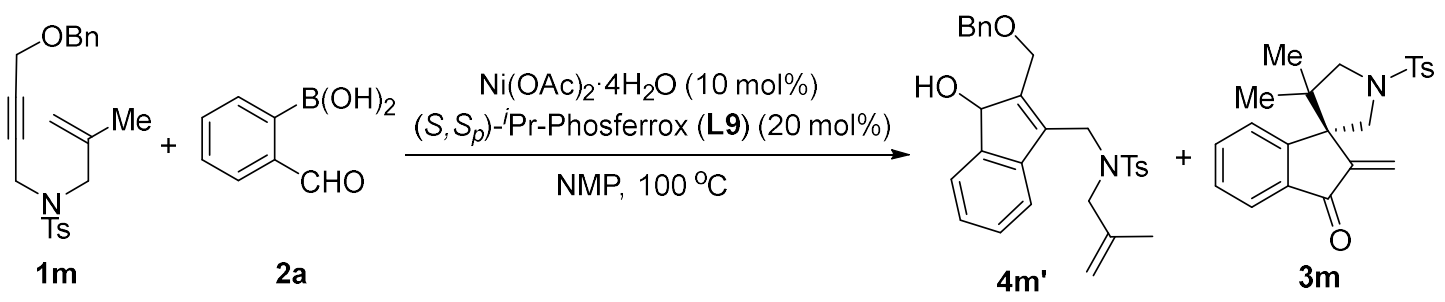

$3 \mathrm{~m}$ was prepared according to general procedure using $1 \mathrm{~m}(0.1 \mathrm{mmol}, 38.3 \mathrm{mg})$ and 2a $(0.3 \mathrm{mmol}, 45.0 \mathrm{mg})$ and was purified by silica gel column chromatography (petroleum ether/ethyl acetate $=10 / 1 \sim 5 / 1)$ to obtain $4 \mathrm{~m}^{\prime}(14.7 \mathrm{mg}, 30 \%$ yield) and $3 \mathrm{~m}$ (12.2 mg, 32\% yield). ${ }^{1} \mathrm{H}$ NMR $\left(600 \mathrm{MHz}, \mathrm{CDCl}_{3}\right) \delta$ 7.87-7.78 (m, 3H), $7.56(\mathrm{t}, J=7.5$ $\mathrm{Hz}, 1 \mathrm{H}), 7.44(\mathrm{t}, J=7.4 \mathrm{~Hz}, 1 \mathrm{H}), 7.40(\mathrm{~d}, J=7.9 \mathrm{~Hz}, 2 \mathrm{H}), 7.30(\mathrm{~d}, J=7.8 \mathrm{~Hz}, 1 \mathrm{H}), 6.26$ (s, 1H), $5.21(\mathrm{~s}, 1 \mathrm{H}), 3.84(\mathrm{~d}, J=10.1 \mathrm{~Hz}, 1 \mathrm{H}), 3.72(\mathrm{~d}, J=10.1 \mathrm{~Hz}, 1 \mathrm{H}), 3.51(\mathrm{~d}, J=$ 10.2 Hz, 1H), 3.38 (d, J = 10.2 Hz, 1H), 2.48 (s, 3H), 0.69 (s, 3H), 0.60 (s, 3H); ${ }^{13} \mathrm{C}$ NMR $\left(151 \mathrm{MHz}, \mathrm{CDCl}_{3}\right) \delta 192.2,151.3,148.5,143.9,137.1,135.0,134.4,129.9$, $128.7,127.3,125.5,124.2,119.1,59.5,57.0,55.7,45.0,23.5,22.8,21.6$;

HRMS: (ESI) calcd for $\mathrm{C}_{22} \mathrm{H}_{24} \mathrm{NO}_{3} \mathrm{~S}^{+}[\mathrm{M}+\mathrm{H}]^{+} 382.1471$; found 382.1461.

The enantiomeric purity was established by HPLC analysis using a chiral column: OD$\mathrm{H}$ column, $30^{\circ} \mathrm{C}, n$-Hexane/i-Propanol $=90 / 10$ as eluent, $254 \mathrm{~nm}, 1 \mathrm{~mL} / \mathrm{min}$. $\mathrm{tR}=17$ $\min$ (minor), 18 min (major).

Optical Rotation: $[\alpha]_{D}^{28}-32.3$ (c 5.0, MeCN) for $98 \%$ ee.

Absolute stereochemistry was determined through analogy with $\mathbf{3 a}$. 
<Chromatogram>

mAU

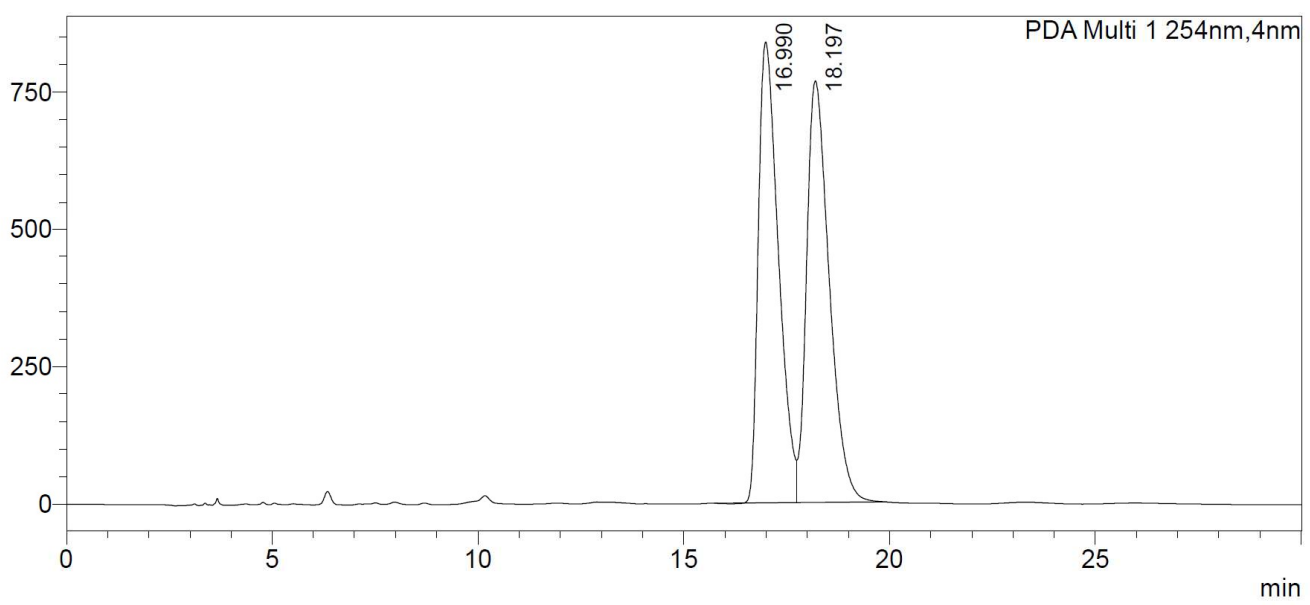

<Peak Table>

PDA Ch1 254nm

Peak\# Ret. Time

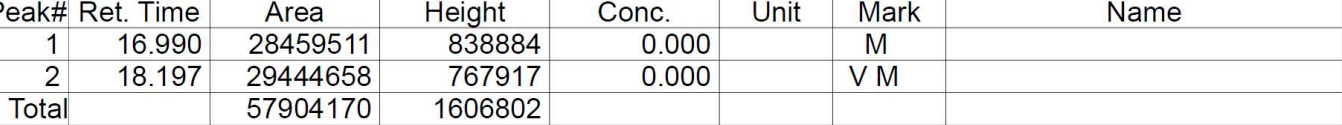

\section{<Chromatogram>}

mAU

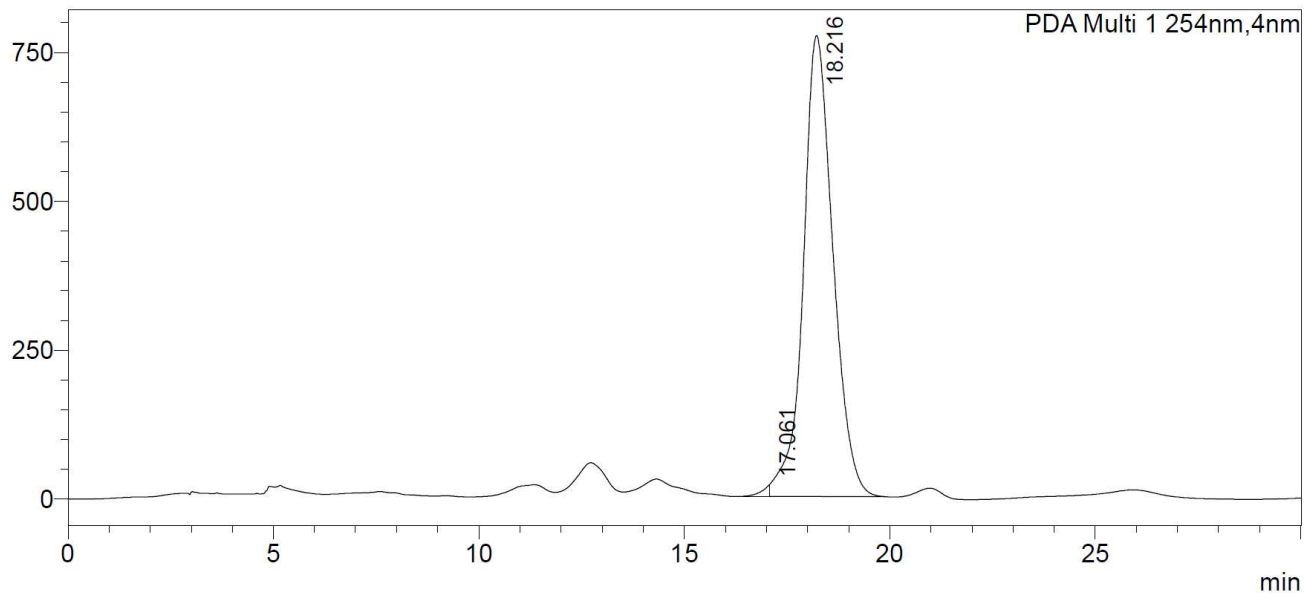

<Peak Table>

PDA Ch1 254nm

\begin{tabular}{|r|r|r|r|r|r|r|r|}
\hline Peak\# Ret. Time & \multicolumn{1}{|c|}{ Area } & Height & Conc. & Unit & Mark & Name \\
\hline 1 & 17.061 & 251714 & 19085 & 0.000 & & $\mathrm{M}$ & \\
\hline 2 & 18.216 & 39011797 & 774085 & 0.000 & & $\mathrm{~V} \mathrm{M}$ & \\
\hline Total & & 39263511 & 793169 & & & & \\
\hline
\end{tabular}




\section{4',4'-dimethyl-1'-tosylspiro[indene-1,3'-pyrrolidine]-3,5'(2H)-dione (3n)}

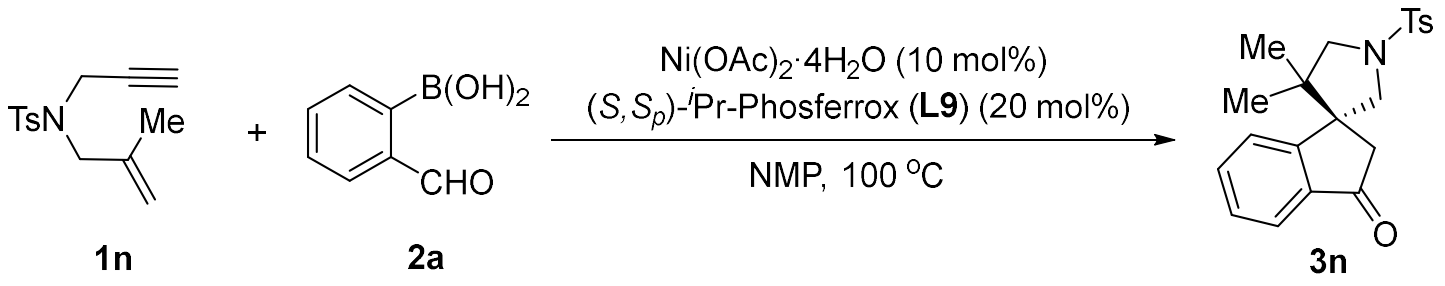

3n was prepared according to general procedure using $1 \mathrm{n}(0.1 \mathrm{mmol}, 26.3 \mathrm{mg})$ and 2a $(0.3 \mathrm{mmol}, 45.0 \mathrm{mg})$ and was purified by silica gel column chromatography (petroleum ether/ethyl acetate $=10 / 1 \sim 5 / 1)$ to obtain $3 \mathrm{n}(11.4 \mathrm{mg}, 31 \%$ yield $)$.

${ }^{1} \mathrm{H}$ NMR $\left(400 \mathrm{MHz}, \mathrm{CDCl}_{3}\right) \delta 7.95(\mathrm{~d}, J=8.4 \mathrm{~Hz}, 2 \mathrm{H}), 7.76-7.70(\mathrm{~m}, 1 \mathrm{H}), 7.45-7.36(\mathrm{~m}$, 4H), $6.96(\mathrm{~d}, J=7.5 \mathrm{~Hz}, 1 \mathrm{H}), 4.02(\mathrm{~s}, 2 \mathrm{H}), 2.78(\mathrm{~d}, J=19.0 \mathrm{~Hz}, 1 \mathrm{H}), 2.54(\mathrm{~d}, J=19.0$ $\mathrm{Hz}, 1 \mathrm{H}), 2.49$ (s, 3H), $1.11(\mathrm{~s}, 3 \mathrm{H}), 0.75(\mathrm{~s}, 3 \mathrm{H})$;

${ }^{13} \mathrm{C}$ NMR $\left(151 \mathrm{MHz}, \mathrm{CDCl}_{3}\right) \delta 202.2,176.9,155.6,145.6,135.7,135.4,134.7,129.8$, $129.1,128.1,124.0,55.2,49.5,48.2,42.6,21.7,21.5,18.5$;

HRMS: (ESI) calcd for $\mathrm{C}_{21} \mathrm{H}_{22} \mathrm{NO}_{4} \mathrm{~S}^{+}[\mathrm{M}+\mathrm{H}]^{+} 384.1264$; found 384.1256.

The enantiomeric purity was established by HPLC analysis using a chiral column: AD$\mathrm{H}$ column, $30^{\circ} \mathrm{C}, n$-Hexane/i-Propanol $=85 / 15$ as eluent, $254 \mathrm{~nm}, 1 \mathrm{~mL} / \mathrm{min}$. tR $=18$ $\min$ (major), 21 min (minor).

Optical Rotation: $[\alpha]_{\mathrm{D}}^{28}-42.4$ (c $5.0, \mathrm{MeCN}$ ) for $19 \%$ ee.

Absolute stereochemistry was determined through analogy with $\mathbf{3 a}$. 
$\mathrm{mAU}$

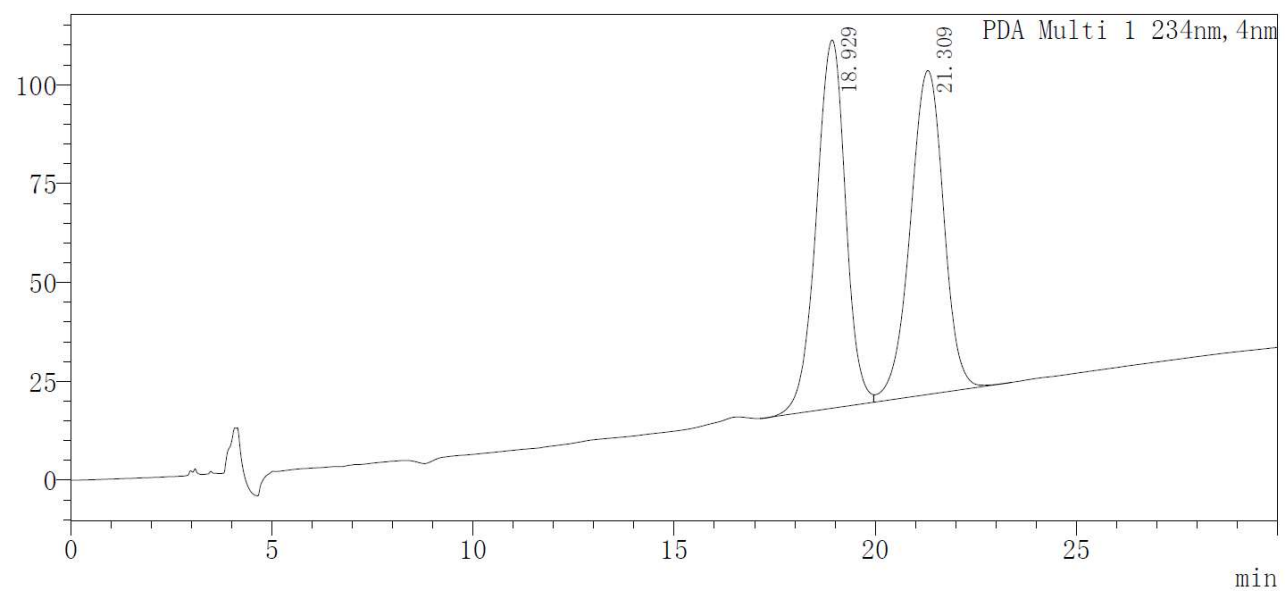

〈峰表〉

PDA Ch1 234nm

\begin{tabular}{|l|l|l|l|l|l|l|}
\hline 峰号 & 保留时间 & 面积 & 高度 & 浓度 & 浓度单位 & 标记多 \\
\hline
\end{tabular}

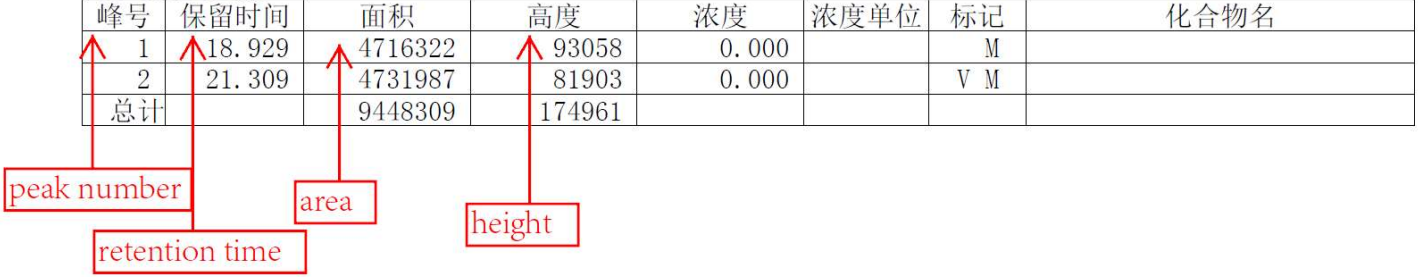

$\mathrm{mAU}$

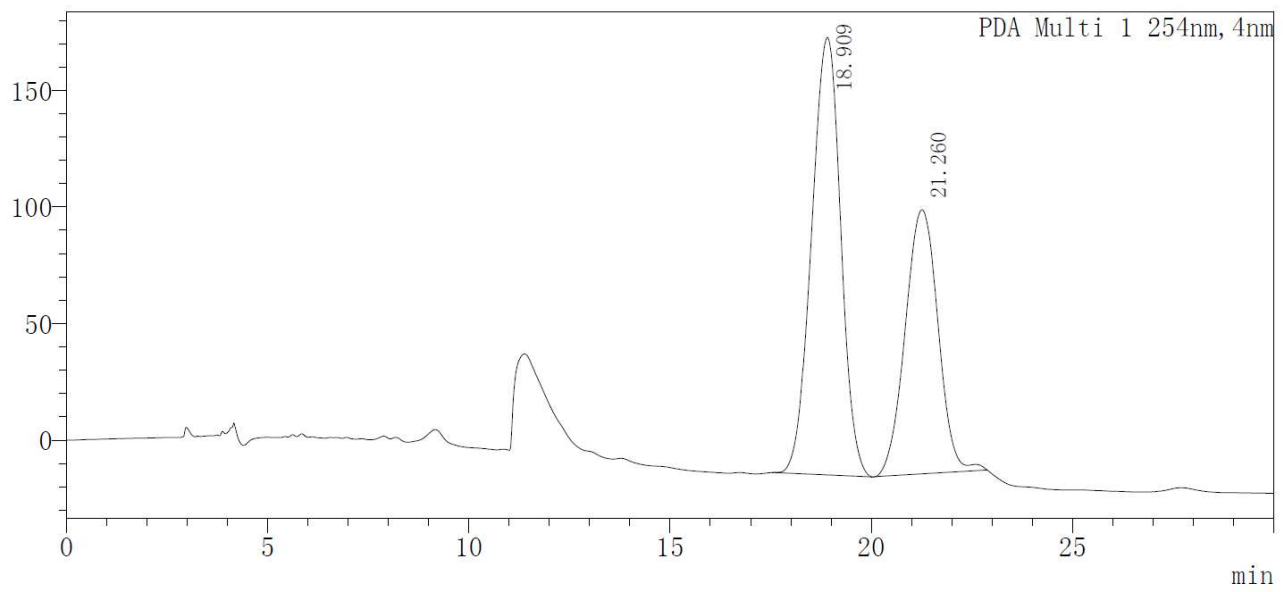

〈峰表〉

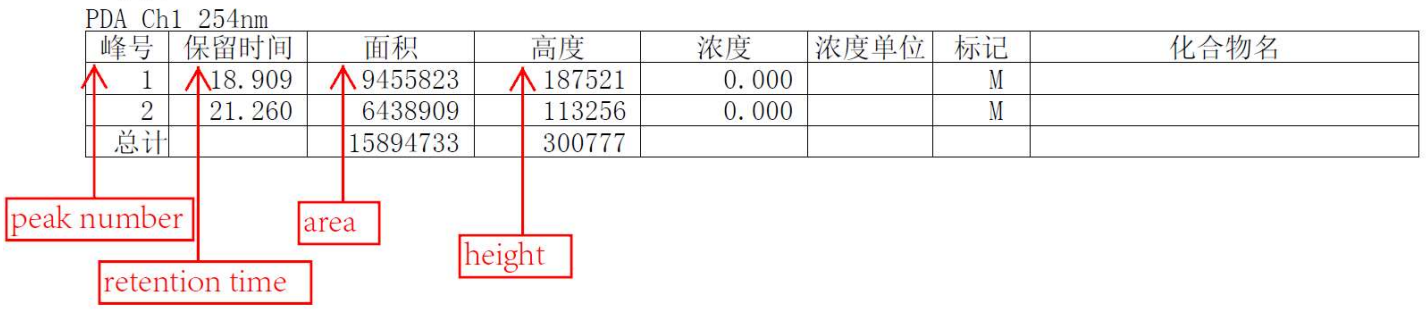


(1R,2S)-4',4'-dimethyl-2-phenyl-1'-tosylspiro[indene-1,3'-pyrrolidine]-3,5'(2H)-

dione (30)

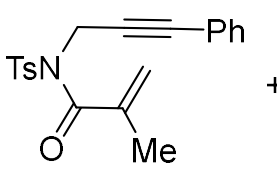

10

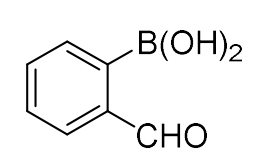

2a

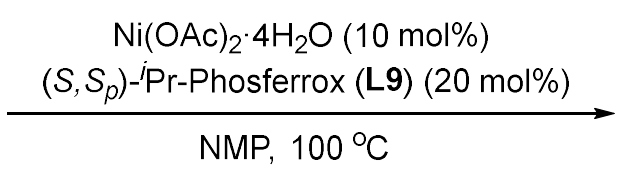

NMP, $100^{\circ} \mathrm{C}$

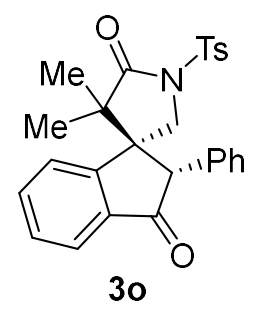

3o was prepared according to general procedure using $10(0.1 \mathrm{mmol}, 35.3 \mathrm{mg})$ and 2a $(0.3 \mathrm{mmol}, 45.0 \mathrm{mg})$ and was purified by silica gel column chromatography (petroleum ether/ethyl acetate $=10 / 1 \sim 5 / 1)$ to obtain $30(37.7 \mathrm{mg}, 82 \%$ yield).

${ }^{1} \mathrm{H} \mathrm{NMR}\left(400 \mathrm{MHz}, \mathrm{CDCl}_{3}\right)$ ठ 7.93-7.87 (m, 2H), 7.86-7.81 (m, 1H), 7.51-7.42 (m, 2H), 7.39-7.35 (m, 2H), 7.34-7.30 (m, 3H), 7.08-7.02 (m, 1H), 6.98-6.88 (m, 2H), $3.85(\mathrm{~s}$, 1H), $3.80(\mathrm{~d}, J=10.8 \mathrm{~Hz}, 1 \mathrm{H}), 3.52(\mathrm{~d}, J=10.8 \mathrm{~Hz}, 1 \mathrm{H}), 2.48(\mathrm{~s}, 3 \mathrm{H}), 1.23(\mathrm{~s}, 3 \mathrm{H})$, $0.68(\mathrm{~s}, 3 \mathrm{H})$;

${ }^{13} \mathrm{C}$ NMR $\left(151 \mathrm{MHz}, \mathrm{CDCl}_{3}\right) \delta 203.9,176.6,156.5,145.5,136.8,136.1,135.9,134.7$, $129.8,129.3,129.0,128.2,128.1$, 124.4, 124.0, 57.5, 53.6, 52.7, 50.1, 22.2, 21.8, 18.7 ; HRMS: (ESI) calcd for $\mathrm{C}_{27} \mathrm{H}_{26} \mathrm{NO}_{4} \mathrm{~S}^{+}[\mathrm{M}+\mathrm{H}]^{+} 460.1577$; found 460.1576 .

The enantiomeric purity was established by HPLC analysis using a chiral column: OD$\mathrm{H}$ column, $30^{\circ} \mathrm{C}, n$-Hexane $/ i-$ Propanol $=80 / 20$ as eluent, $254 \mathrm{~nm}, 1 \mathrm{~mL} / \mathrm{min}$. tR $=10$ $\min$ (major), 15 min (minor).

Optical Rotation: $[\alpha]_{D}^{28} 23.2$ (c 5.0, MeCN) for $94 \%$ ee.

Absolute stereochemistry was determined through analogy with $\mathbf{3 a}$. 
<Chromatogram>

mAU

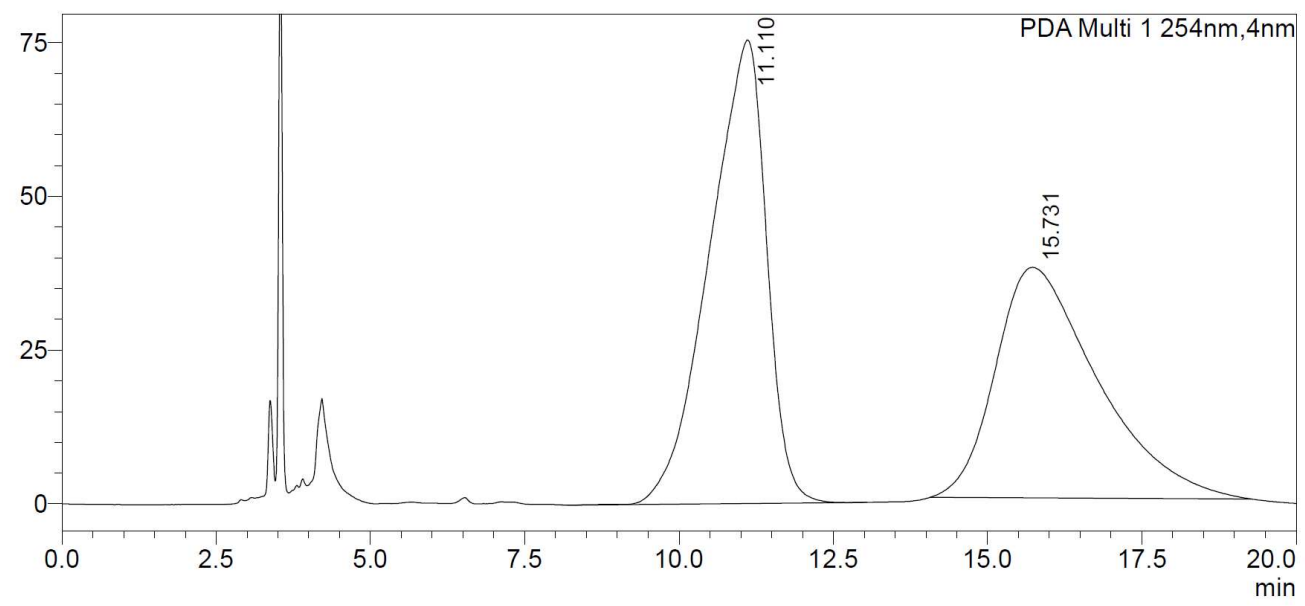

<Peak Table>

PDA Ch1 254nm

\begin{tabular}{|r|r|r|r|r|r|r|r|}
\hline Peak\# Ret. Time & Area & Height & Conc. & Unit & Mark & Name \\
\hline 1 & 11.110 & 4848563 & 75316 & 0.000 & & $\mathrm{M}$ & \\
\hline 2 & 15.731 & 4320691 & 37453 & 0.000 & & $\mathrm{M}$ & \\
\hline Total & & 9169254 & 112770 & & & & \\
\hline
\end{tabular}

\section{$<$ Chromatogram>}

mAU

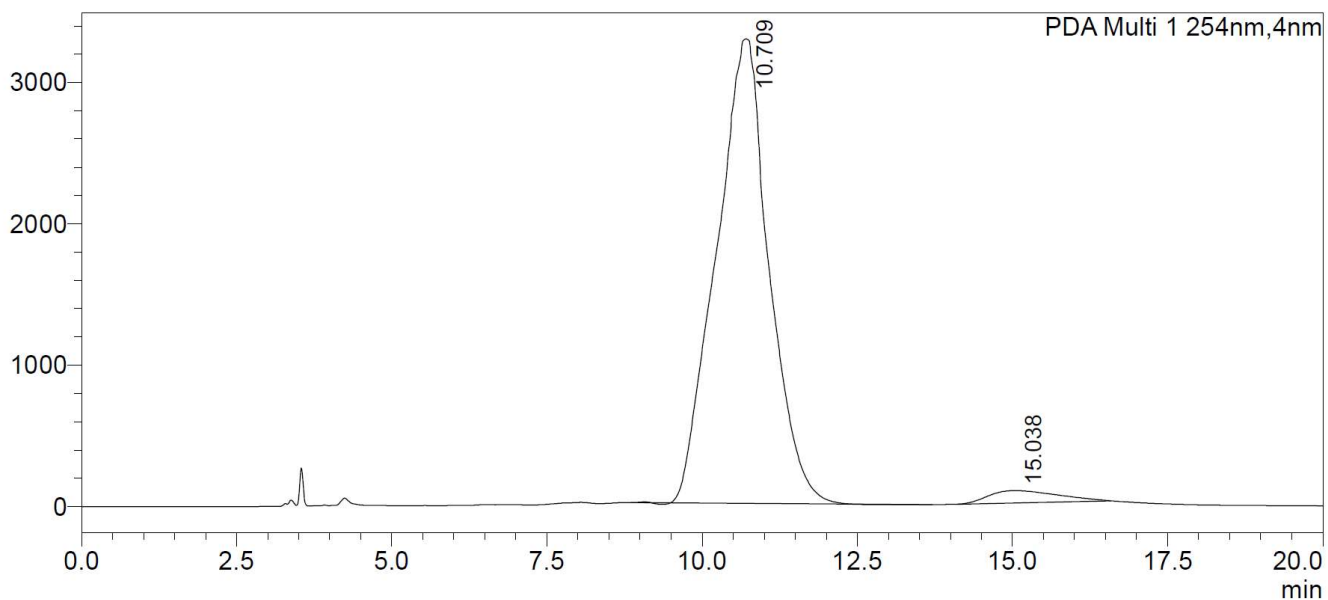

<Peak Table>

PDA Ch1 254nm

Peak\# Ret. Time Area

Height

Height Conc.

0.000

6696507

87326

200499520

3373160 


\section{(1S,2R,4'R)-4'-methyl-2-phenyl-1'-tosylspiro[indene-1,3'-pyrrolidin]-3(2H)-one}

(3p)

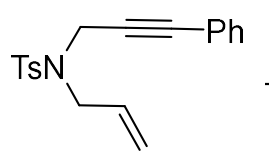

$1 \mathrm{p}$<smiles>O=Cc1ccccc1Br</smiles>

$2 a$
$\mathrm{Ni}(\mathrm{OAc})_{2} \cdot 4 \mathrm{H}_{2} \mathrm{O}(10 \mathrm{~mol} \%)$ $\underset{\left(S, S_{p}\right){ }^{-} \text {Pr-Phosferrox (L9) }(20 \mathrm{~mol} \%)}{\text { NMP } 100^{\circ} \mathrm{C}}$

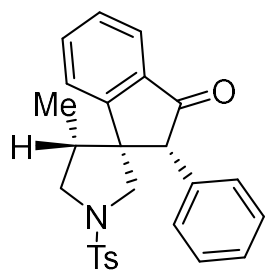

$3 p$

$3 p$ was prepared according to general procedure using $1 \mathrm{p}(0.1 \mathrm{mmol}, 32.5 \mathrm{mg})$ and 2a $(0.3 \mathrm{mmol}, 45.0 \mathrm{mg})$ and was purified by silica gel column chromatography (petroleum ether/ethyl acetate $=10 / 1 \sim 5 / 1)$ to obtain $3 p(34.0 \mathrm{mg}, 79 \%$ yield $)$. ${ }^{1} \mathrm{H}$ NMR $\left(400 \mathrm{MHz}, \mathrm{CDCl}_{3}\right) \delta 7.84(\mathrm{~d}, J=7.6 \mathrm{~Hz}, 1 \mathrm{H}), 7.72-7.66(\mathrm{~m}, 1 \mathrm{H}), 7.53-7.47(\mathrm{~m}$, 3H), $7.44(\mathrm{~d}, J=7.8 \mathrm{~Hz}, 1 \mathrm{H}), 7.26-7.15(\mathrm{~m}, 5 \mathrm{H}), 6.81-6.75(\mathrm{~m}, 2 \mathrm{H}), 3.80$ (dd, $J=10.1$, $7.6 \mathrm{~Hz}, 1 \mathrm{H}), 3.74(\mathrm{~s}, 1 \mathrm{H}), 3.51(\mathrm{~d}, J=10.3 \mathrm{~Hz}, 1 \mathrm{H}), 3.25(\mathrm{~d}, J=10.2 \mathrm{~Hz}, 1 \mathrm{H}), 3.03(\mathrm{t}$, $J=10.2 \mathrm{~Hz}, 1 \mathrm{H}), 2.45(\mathrm{~s}, 3 \mathrm{H}), 2.39-2.32(\mathrm{~m}, 1 \mathrm{H}), 0.76(\mathrm{~d}, J=6.9 \mathrm{~Hz}, 3 \mathrm{H})$;

${ }^{13} \mathrm{C}$ NMR $\left(101 \mathrm{MHz}, \mathrm{CDCl}_{3}\right) \delta 204.5,155.5,143.3,137.4,137.0,135.8,134.4,129.6$, $129.0,128.9,128.8,127.4,127.2,124.1,123.8,58.2,56.3,54.2,53.4,46.3,21.5,10.7$ HRMS: (ESI) calcd for $\mathrm{C}_{26} \mathrm{H}_{26} \mathrm{NO}_{3} \mathrm{~S}^{+}[\mathrm{M}+\mathrm{H}]^{+}$432.1628; found 432.1636.

The enantiomeric purity was established by HPLC analysis using a chiral column: OD$\mathrm{H}$ column, $30^{\circ} \mathrm{C}, n$-Hexane/i-Propanol $=80 / 20$ as eluent, $254 \mathrm{~nm}, 1 \mathrm{~mL} / \mathrm{min} . \mathrm{tR}=12$ $\min$ (major), 17 min (minor).

Optical Rotation: $[\alpha]_{D}^{28} 15.4$ (c 5.0, MeCN) for $90 \%$ ee.

Absolute stereochemistry was determined through analogy with $\mathbf{3 u}$. 
$<$ Chromatogram>

mAU

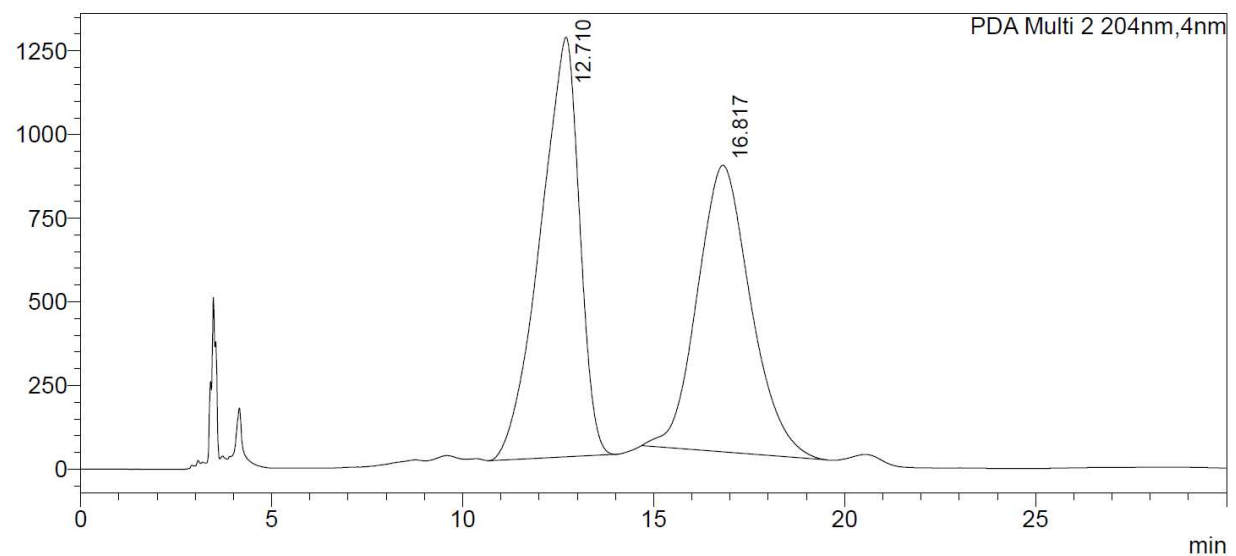

<Peak Table>

PDA Ch2 204nm

Peak\# Ret. Time

\begin{tabular}{r|r|r}
1 & 12.710 & Area \\
1 & 16450390
\end{tabular}

\begin{tabular}{|l|l|r|}
\hline 2 & 16.817 & 83326570 \\
\hline
\end{tabular}

\begin{tabular}{c|r} 
Total & 172776959
\end{tabular}

\begin{tabular}{|r|r|r|r|r|r|}
\hline \multicolumn{1}{|c|}{ Height } & \multicolumn{1}{|c|}{ Conc. } & Unit & Mark & Name \\
\hline 0 & 1253743 & 51.772 & & $\mathrm{M}$ & \\
\hline 9 & 2110406 & 48.228 & & $\mathrm{M}$ & \\
\hline
\end{tabular}

$<$ Chromatogram>

mAU

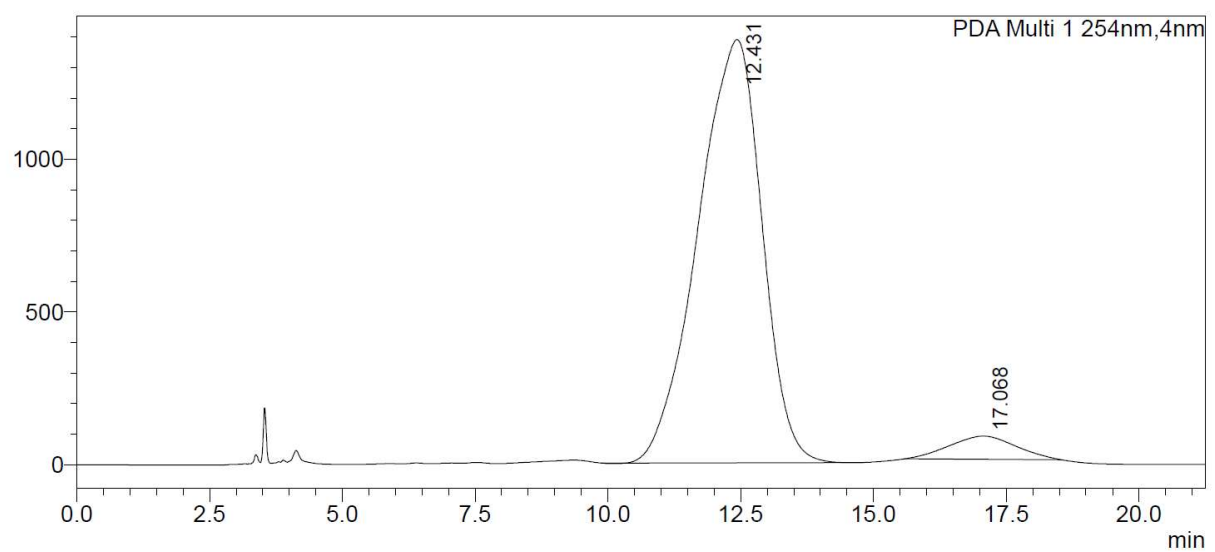

<Peak Table>

PDA Ch1 254nm

\begin{tabular}{|r|r|r|r|r|r|r|r|}
\hline Peak\# Ret. Time & \multicolumn{1}{|c|}{ Area } & Height & Conc. & Unit & Mark & Name \\
\hline 1 & 12.431 & 116162777 & 1385286 & 0.000 & & $\mathrm{M}$ & \\
\hline 2 & 17.068 & 6609693 & 75598 & 0.000 & & $\mathrm{M}$ & \\
\hline Total & & 122772471 & 1460884 & & & & \\
\end{tabular}


$\left(1 R, 2 R, 4^{\prime} R\right)-4^{\prime}-$ methyl-2-phenyl-1'-tosylspiro[indene-1,3'-pyrrolidine]-3,5'(2H)-

dione (3q)

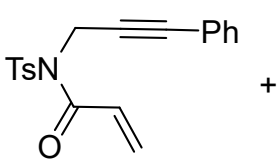

$1 \mathrm{q}$<smiles>O=Cc1ccccc1Br</smiles>

$2 a$

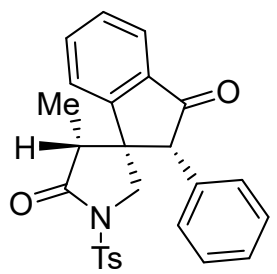

$3 q$

$\mathbf{3 q}$ was prepared according to general procedure using $\mathbf{1 q}(0.1 \mathrm{mmol}, 33.9 \mathrm{mg})$ and 2a $(0.3 \mathrm{mmol}, 45.0 \mathrm{mg})$ and was purified by silica gel column chromatography (petroleum ether/ethyl acetate $=10 / 1 \sim 5 / 1)$ to obtain $3 q(23.1 \mathrm{mg}, 52 \%$ yield).

${ }^{1} \mathrm{H}$ NMR $\left(400 \mathrm{MHz}, \mathrm{CDCl}_{3}\right)$ ठ 7.91-7.86 (m, 1H), 7.75-7.71 (m, 1H), 7.70-7.66 (m, 2H), 7.59-7.53 (m, 2H), 7.32-7.28 (m, 2H), 7.27-7.19 (m, 3H), 6.88-6.81 (m, 2H), 3.92 (d, J $=10.7 \mathrm{~Hz}, 1 \mathrm{H}), 3.90(\mathrm{~s}, 1 \mathrm{H}), 3.82(\mathrm{~d}, J=10.7 \mathrm{~Hz}, 1 \mathrm{H}), 3.00(\mathrm{q}, J=7.1 \mathrm{~Hz}, 1 \mathrm{H}), 2.47$ (s, 3H), $1.11(\mathrm{~d}, J=7.2 \mathrm{~Hz}, 3 \mathrm{H})$;

${ }^{13} \mathrm{C}$ NMR $\left(151 \mathrm{MHz}, \mathrm{CDCl}_{3}\right) \delta$ 203.2, 172.8, 154.2, 145.2, 136.8, 136.1, 135.7, 135.0, 129.6, 129.6, 129.4, 129.2, 128.2, 128.0, 124.6, 123.5, 57.8, 52.6, 51.3, 50.3, 21.8, 8.9;

HRMS: (ESI) calcd for $\mathrm{C}_{26} \mathrm{H}_{24} \mathrm{NO}_{4} \mathrm{~S}^{+}[\mathrm{M}+\mathrm{H}]^{+}$446.1421; found 446.1420.

The enantiomeric purity was established by HPLC analysis using a chiral column: IF column, $30^{\circ} \mathrm{C}, n$-Hexane/i-Propanol $=60 / 40$ as eluent, $254 \mathrm{~nm}, 0.5 \mathrm{~mL} / \mathrm{min}$. tR $=23$ $\min$ (minor), 25 min (major).

Optical Rotation: $[\alpha]_{D}^{28}-26.7$ (c 5.0, MeCN) for $90 \%$ ee.

Absolute stereochemistry was determined through analogy with $\mathbf{3 u}$. 
$<$ Chromatogram >

mAU

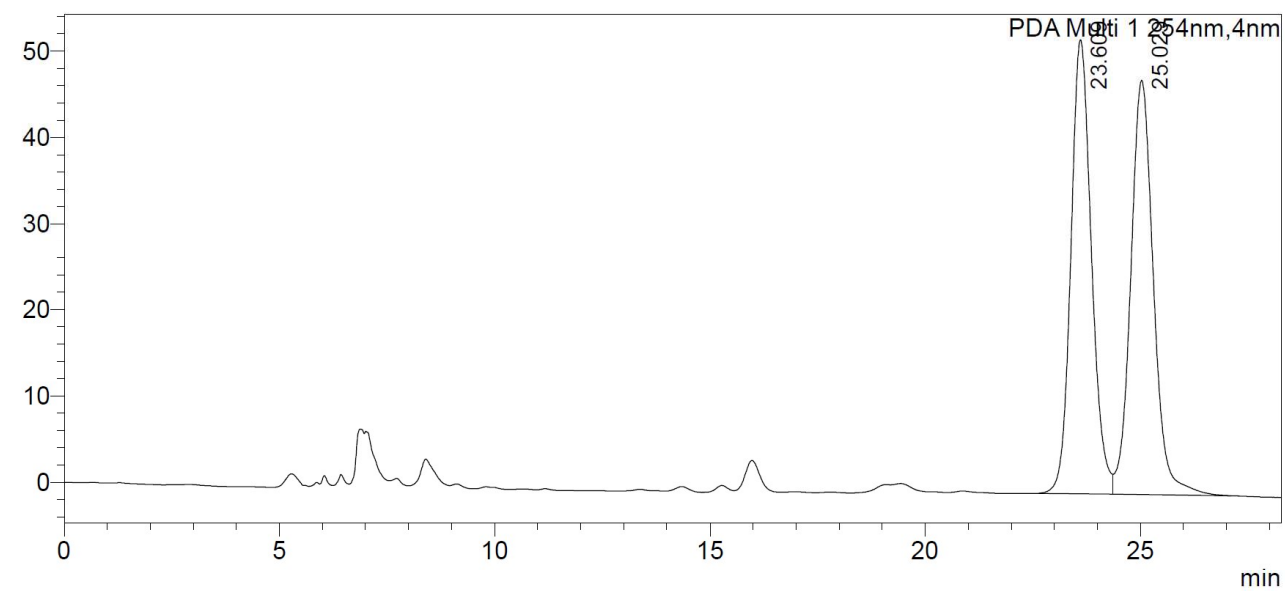

<Peak Table>

PDA Ch1 254nm

\begin{tabular}{|r|r|r|r|r|r|r|r|}
\hline Peak\# Ret. Time & Area & Height & Conc. & Unit & Mark & Name \\
\hline 1 & 23.609 & 1747370 & 52630 & 0.000 & & & \\
\hline 2 & 25.029 & 1762011 & 48025 & 0.000 & & V & \\
\hline Total & & 3509381 & 100655 & & & & \\
\end{tabular}

\section{$<$ Chromatogram>}

mAU

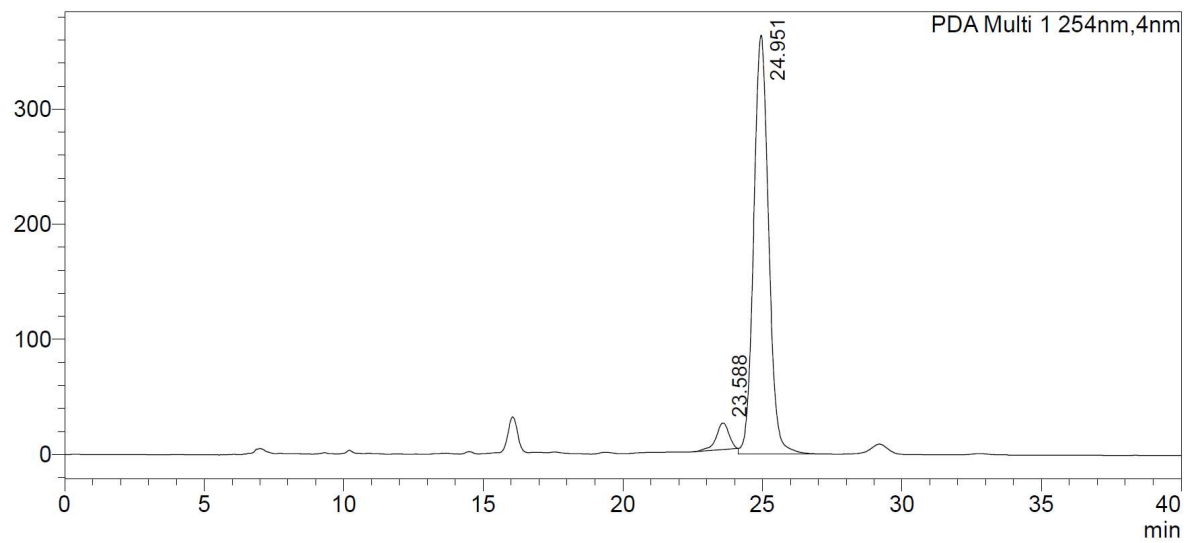

<Peak Table>

PDA Ch1 254nm

Peak\# Ret. Time

\begin{tabular}{|c|r|c|}
\hline 1 & 23.588 & Area \\
\hline & 24.951 & 1357538
\end{tabular}

Area
747538
13525898

Height

Conc.

\begin{tabular}{r|r|r|r|}
23143 & 0.000 & Unit & M \\
\hline 363751 & 0.000 &
\end{tabular}

\begin{tabular}{|l|r|r|r|}
\hline Total & 24.951 & 13525898 & 363751 \\
\hline
\end{tabular} 
$\left(1 R, 2 R, 4^{\prime} R\right)-4$ '-hexyl-4'-methyl-2-phenyl-1'-tosylspiro[indene-1,3'-pyrrolidine]-

\section{$3,5^{\prime}(2 H)$-dione $(3 r)$}

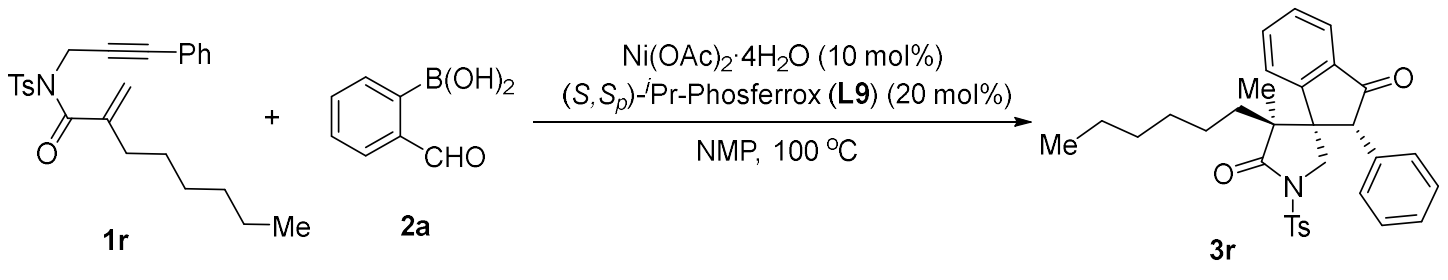

$3 \mathbf{r}$ was prepared according to general procedure using $1 \mathbf{r}(0.1 \mathrm{mmol}, 42.3 \mathrm{mg})$ and $2 \mathrm{a}$

$(0.3 \mathrm{mmol}, 45.0 \mathrm{mg})$ and was purified by silica gel column chromatography (petroleum ether/ethyl acetate $=10 / 1 \sim 5 / 1)$ to obtain $3 r(33.3 \mathrm{mg}, 63 \%$ yield $)$.

${ }^{1} \mathrm{H} \mathrm{NMR}\left(400 \mathrm{MHz}, \mathrm{CDCl}_{3}\right)$ ס 7.90-7.86 (m, 2H), 7.85-7.83 (m, 1H), 7.52-7.43 (m, 2H), 7.38-7.34 (m, 2H), 7.33-7.28 (m, 3H), 7.13-7.08 (m, 1H), 6.91 (bs, 2H), $3.94(\mathrm{~s}, 1 \mathrm{H})$, $3.74(\mathrm{~d}, J=10.9 \mathrm{~Hz}, 1 \mathrm{H}), 3.46(\mathrm{~d}, J=10.9 \mathrm{~Hz}, 1 \mathrm{H}), 3.06(\mathrm{~s}, 3 \mathrm{H}), 1.44-1.32(\mathrm{~m}, 1 \mathrm{H})$, 1.31-1.26 (m, 1H), 1.10-1.03 (m, 2H), 1.00-0.93 (m, 2H), 0.91-0.77 (m, 4H), $0.74(\mathrm{t}, J$ $=7.3 \mathrm{~Hz}, 3 \mathrm{H})$;

${ }^{13} \mathrm{C}$ NMR $\left(151 \mathrm{MHz}, \mathrm{CDCl}_{3}\right) \delta$ 204.2, 176.7, 156.1, 145.5, 137.2, 136.3, 135.9, 134.8, $129.8,129.3,128.2,128.1,124.4,124.3,58.2,54.1,52.6,52.0,33.0,31.1$, 29.6, 22.7, 22.3, 21.8, 18.2, 13.9;

HRMS: (ESI) calcd for $\mathrm{C}_{32} \mathrm{H}_{36} \mathrm{NO}_{4} \mathrm{~S}^{+}[\mathrm{M}+\mathrm{H}]^{+}$530.2360; found 530.2350.

The enantiomeric purity was established by HPLC analysis using a chiral column: OD$\mathrm{H}$ column, $30^{\circ} \mathrm{C}, n$-Hexane/i-Propanol $=90 / 10$ as eluent, $254 \mathrm{~nm}, 1 \mathrm{~mL} / \mathrm{min} . \mathrm{tR}=9 \mathrm{~min}$ (major), 13 min (minor).

Optical Rotation: $[\alpha]_{D}{ }^{28}-50.8$ (c 5.0, MeCN) for $96 \%$ ee.

Absolute stereochemistry was determined through analogy with $\mathbf{3 u}$. 
<Chromatogram>

mAU

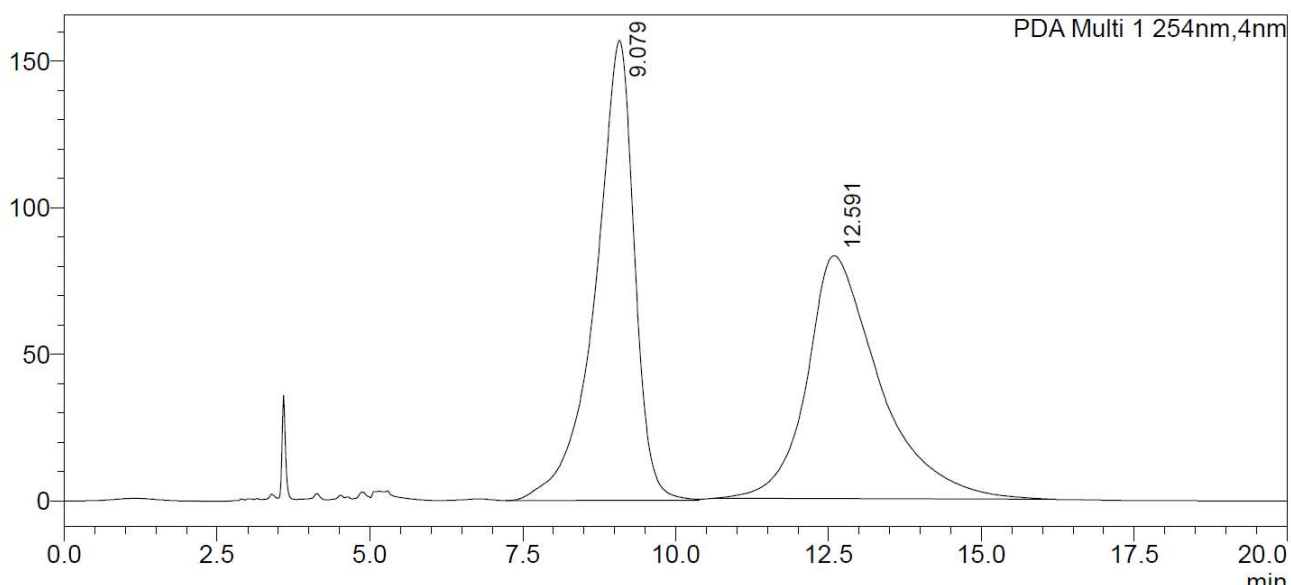

<Peak Table>

PDA Ch1 254nm

\begin{tabular}{|r|r|r|r|r|r|r|r|}
\hline Peak\# Ret. Time & \multicolumn{1}{|c|}{ Area } & Height & \multicolumn{1}{c|}{ Conc. } & Unit & Mark & Name \\
\hline 1 & 9.079 & 6949088 & 156914 & 0.000 & & & \\
\hline 2 & 12.591 & 6855622 & 82799 & 0.000 & & $\mathrm{M}$ & \\
\hline Total & & 13804710 & 239713 & & & & \\
\hline
\end{tabular}

<Chromatogram>

mAU

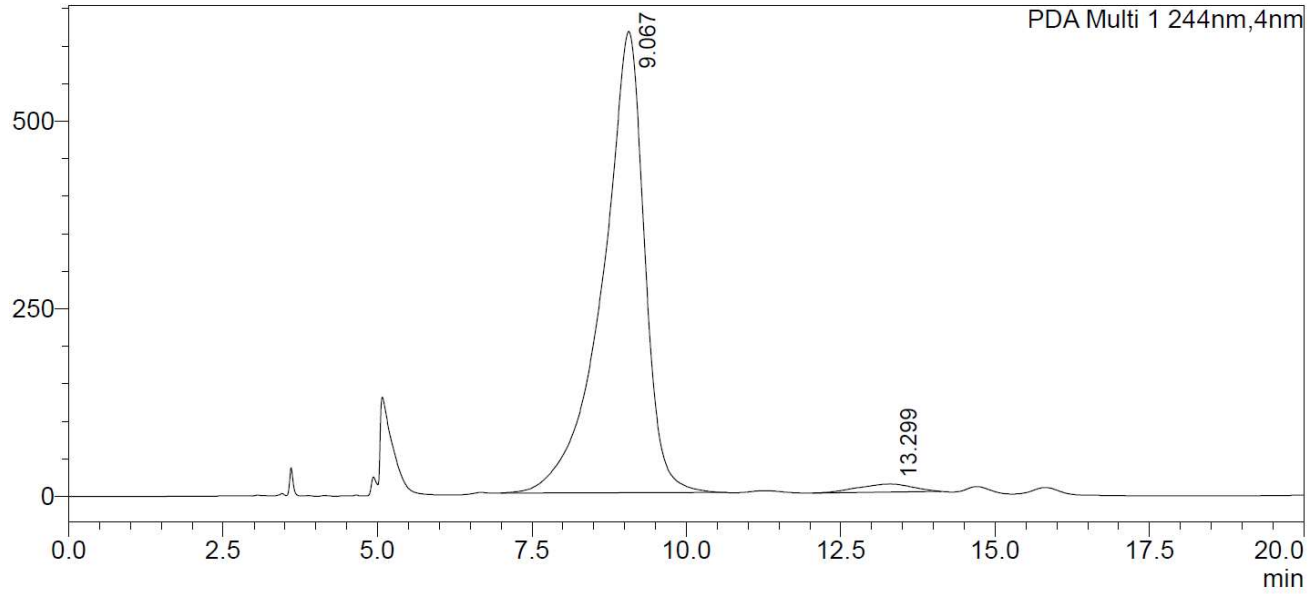

<Peak Table>

PDA Ch1 244nm

Peak\# Ret. Time

Area

Height

Conc.

Unit

Mark

Name

\begin{tabular}{|r|r|}
\hline 1 & 9.067 \\
\hline 2 & 13.299 \\
\hline
\end{tabular}

44994

615082

0.000

29625641

625712

0.000 
$(1 R, 2 R, 4 ' R)-4$ '-benzyl-4'--methyl-2-phenyl-1'-tosylspiro[indene-1,3'-pyrrolidine]-

\section{$3,5^{\prime}(2 \mathrm{H})$-dione $(3 \mathrm{~s})$}

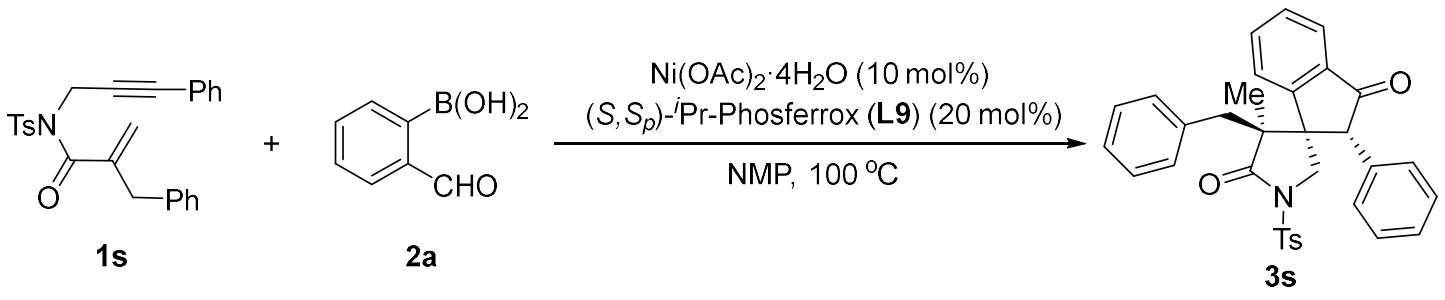

3s was prepared according to general procedure using $1 \mathrm{~s}(0.1 \mathrm{mmol}, 42.9 \mathrm{mg})$ and $2 \mathrm{a}$ $(0.3 \mathrm{mmol}, 45.0 \mathrm{mg}$ ) and was purified by silica gel column chromatography (petroleum ether/ethyl acetate $=10 / 1 \sim 5 / 1)$ to obtain 3 s $(31.6 \mathrm{mg}, 59 \%$ yield $)$.

${ }^{1} \mathrm{H}$ NMR $\left(600 \mathrm{MHz}, \mathrm{CDCl}_{3}\right)$ ठ 7.94-7.86 (m, 3H), 7.60-7.56 (m, 1H), 7.54-7.50 (m, 1H), $7.37(\mathrm{~d}, J=8.0 \mathrm{~Hz}, 2 \mathrm{H}), 7.29-7.26(\mathrm{~m}, 3 \mathrm{H}), 7.19-7.10(\mathrm{~m}, 4 \mathrm{H}), 6.85-6.75(\mathrm{~m}, 2 \mathrm{H}), 6.63-$ $6.58(\mathrm{~m}, 2 \mathrm{H}), 3.73(\mathrm{~d}, J=10.8 \mathrm{~Hz}, 1 \mathrm{H}), 3.50(\mathrm{~d}, J=10.8 \mathrm{~Hz}, 1 \mathrm{H}), 3.36(\mathrm{~s}, 1 \mathrm{H}), 2.87(\mathrm{~d}$, $J=14.4 \mathrm{~Hz}, 1 \mathrm{H}), 2.50$ (s, 3H), $2.44(\mathrm{~d}, J=14.4 \mathrm{~Hz}, 1 \mathrm{H}), 1.24(\mathrm{~s}, 3 \mathrm{H})$; ${ }^{13} \mathrm{C}$ NMR $\left(151 \mathrm{MHz}, \mathrm{CDCl}_{3}\right) \delta$ 203.8, 176.3, 155.8, 145.5, 136.9, 136.6, 135.9, 134.8, $134.7,130.4,129.7,129.5,129.5,129.1,128.3,128.2,127.9,127.1,124.5,124.4$, $57.9,54.2,53.2,52.3,38.2,21.8,19.3$

HRMS: (ESI) calcd for $\mathrm{C}_{33} \mathrm{H}_{30} \mathrm{NO}_{4} \mathrm{~S}^{+}[\mathrm{M}+\mathrm{H}]^{+}$536.1890; found 536.1886.

The enantiomeric purity was established by HPLC analysis using a chiral column: OD$\mathrm{H}$ column, $30^{\circ} \mathrm{C}, n$-Hexane/i-Propanol $=80 / 20$ as eluent, $254 \mathrm{~nm}, 1 \mathrm{~mL} / \mathrm{min} . \mathrm{tR}=10$ min (minor), 13 min (major).

Optical Rotation: $[\alpha]_{\mathrm{D}}^{28}-60.9$ (c 5.0, MeCN) for $97 \%$ ee.

Absolute stereochemistry was determined through analogy with $3 \mathbf{u}$. 
<Chromatogram>

mAU

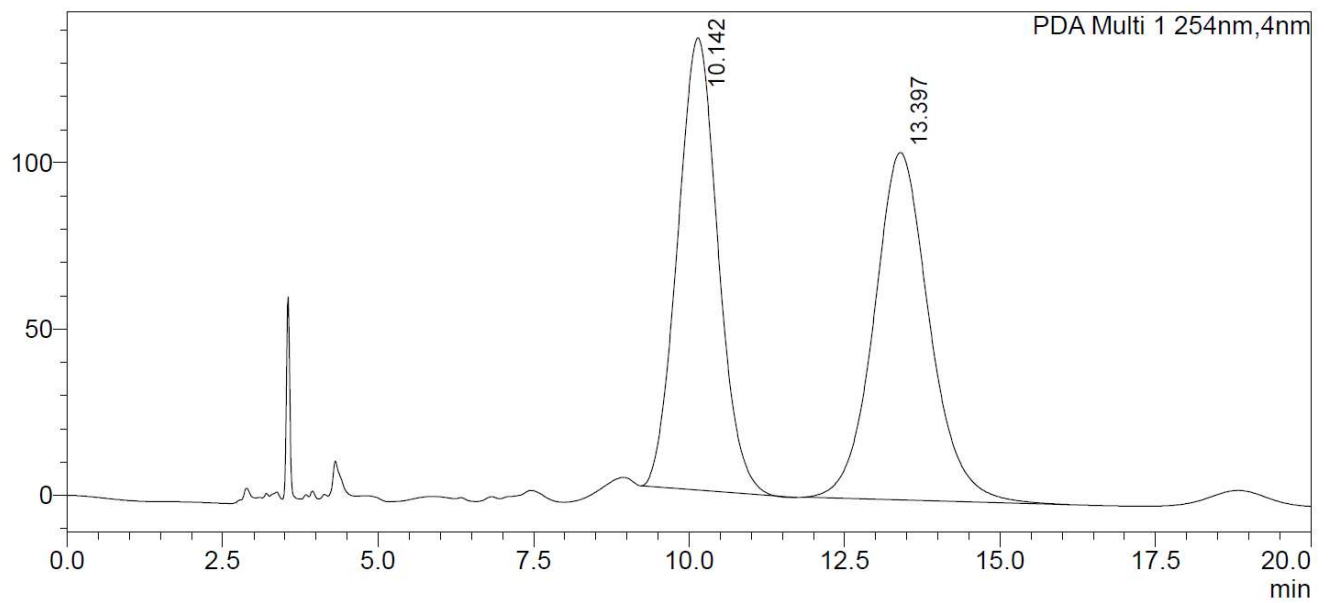

<Peak Table>

PDA Ch1 254nm

Peak\# Ret. Time

\begin{tabular}{|r|r|r|r|r|r|r|r|}
\hline Peak\# Ret. Time & \multicolumn{1}{|c|}{ Area } & Height & Conc. & Unit & Mark & Name \\
\hline 1 & 10.142 & 6151857 & 136013 & 0.000 & & $\mathrm{M}$ & \\
\hline 2 & 13.397 & 6484128 & 104449 & 0.000 & & $\mathrm{M}$ & \\
\hline Total & & 12635985 & 240461 & & & & \\
\end{tabular}

\section{<Chromatogram>}

mAU

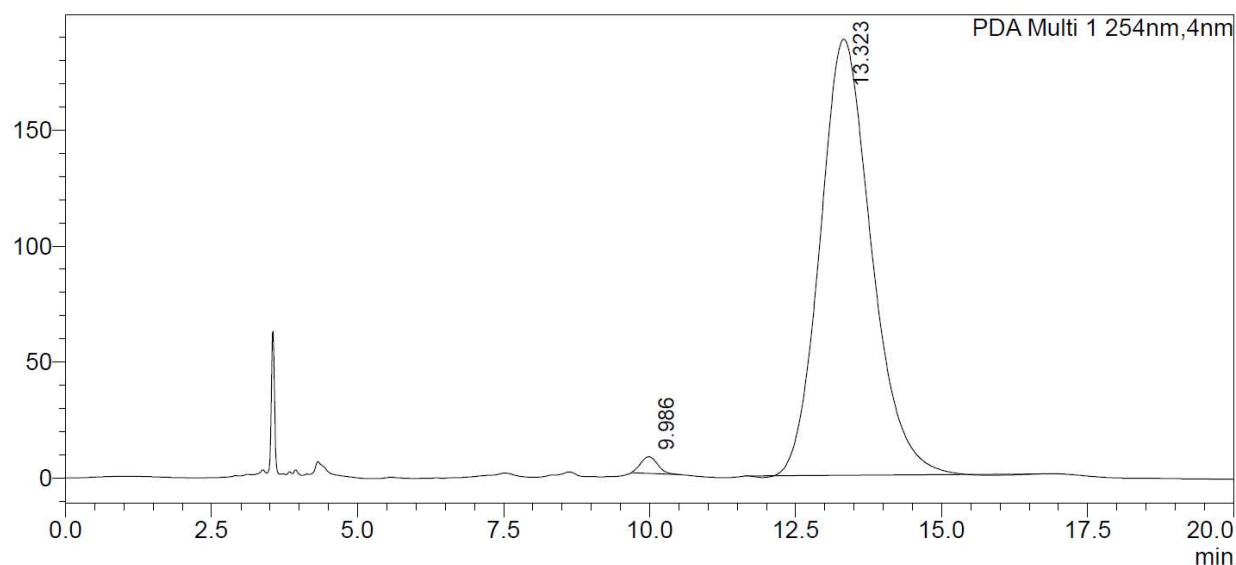

<Peak Table>

PDA Ch1 254nm

\begin{tabular}{|r|r|r|r|r|r|r|r|}
\hline Peak\# Ret. Time & \multicolumn{1}{|c|}{ Area } & \multicolumn{1}{|c|}{ Height } & \multicolumn{1}{c|}{ Conc. } & Unit & Mark & Name \\
\hline 1 & 9.986 & 144820 & 7170 & 0.000 & & $\mathrm{M}$ & \\
\hline 2 & 13.323 & 11457153 & 188006 & 0.000 & & $\mathrm{M}$ & \\
\hline Total & & 11601973 & 195176 & & & & \\
\end{tabular}


$\left(1 R, 2 R, 4^{\prime} R\right)$-methyl 4'-methyl-3-oxo-2-phenyl-1'-tosyl-2,3-dihydrospiro[indene-

\section{1,3 '-pyrrolidine]-4'-carboxylate (3t)}

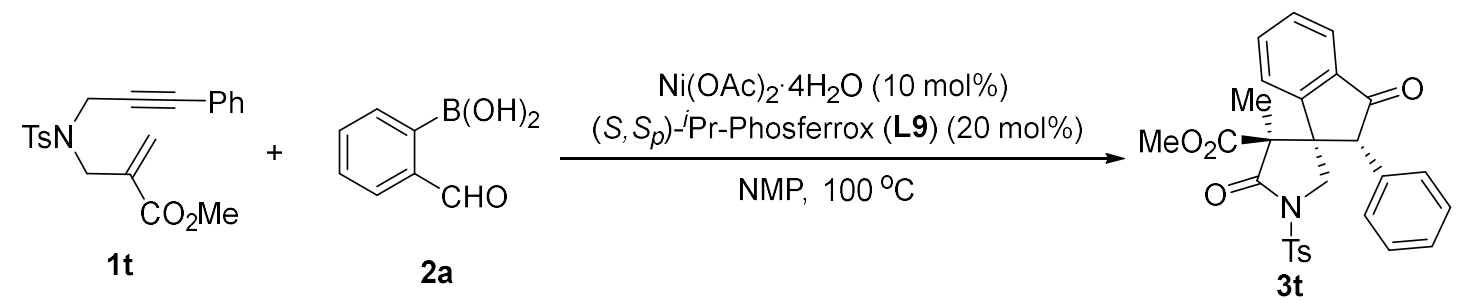

3t was prepared according to general procedure using $\mathbf{1 t}(0.1 \mathrm{mmol}, 38.3 \mathrm{mg})$ and $\mathbf{2 a}$ $(0.3 \mathrm{mmol}, 45.0 \mathrm{mg}$ ) and was purified by silica gel column chromatography (petroleum ether/ethyl acetate $=10 / 1 \sim 5 / 1)$ to obtain $3 t(35.7 \mathrm{mg}, 73 \%$ yield $)$.

${ }^{1} \mathrm{H}$ NMR $\left(600 \mathrm{MHz}, \mathrm{CDCl}_{3}\right)$ ठ 7.82-7.76 (m, 1H), 7.69-7.62 (m, 2H), 7.58-7.45 (m, 2H), 7.38-7.29 (m, 3H), 7.28-7.21 (m, 3H), 6.86-6.75 (m, 2H), $4.23(\mathrm{~s}, 1 \mathrm{H}), 4.22(\mathrm{~d}, J=10.1$ $\mathrm{Hz}, 1 \mathrm{H}), 3.42(\mathrm{~d}, J=11.1 \mathrm{~Hz}, 1 \mathrm{H}), 3.35(\mathrm{~d}, J=10.6 \mathrm{~Hz}, 1 \mathrm{H}), 3.21(\mathrm{~s}, 3 \mathrm{H}), 3.09(\mathrm{~d}, J=$ 10.6 Hz, 1H), $2.48(\mathrm{~s}, 3 \mathrm{H}), 1.22(\mathrm{~s}, 3 \mathrm{H})$;

${ }^{13} \mathrm{C}$ NMR $\left(151 \mathrm{MHz}, \mathrm{CDCl}_{3}\right) \delta 204.0,171.6,155.3,144.0,137.7,136.4,135.7,133.7$, $129.9,129.4,129.1,128.4,127.9,127.3,125.7,123.5,58.6,57.8,55.3,54.9,54.3$, $51.7,22.5,21.6$

HRMS: (ESI) calcd for $\mathrm{C}_{28} \mathrm{H}_{28} \mathrm{NO}_{5} \mathrm{~S}^{+}[\mathrm{M}+\mathrm{H}]^{+}$490.1683; found 490.1680.

The enantiomeric purity was established by HPLC analysis using a chiral column: IF column, $30^{\circ} \mathrm{C}, n$-Hexane/i-Propanol $=60 / 40$ as eluent, $254 \mathrm{~nm}, 1 \mathrm{~mL} / \mathrm{min}$. $\mathrm{tR}=10 \mathrm{~min}$ (major), 20 min (minor).

Optical Rotation: $[\alpha]_{D}^{28}-84.8$ (c 5.0, MeCN) for $90 \%$ ee.

Absolute stereochemistry was determined through analogy with $\mathbf{3 u}$. 
<Chromatogram>

mAU

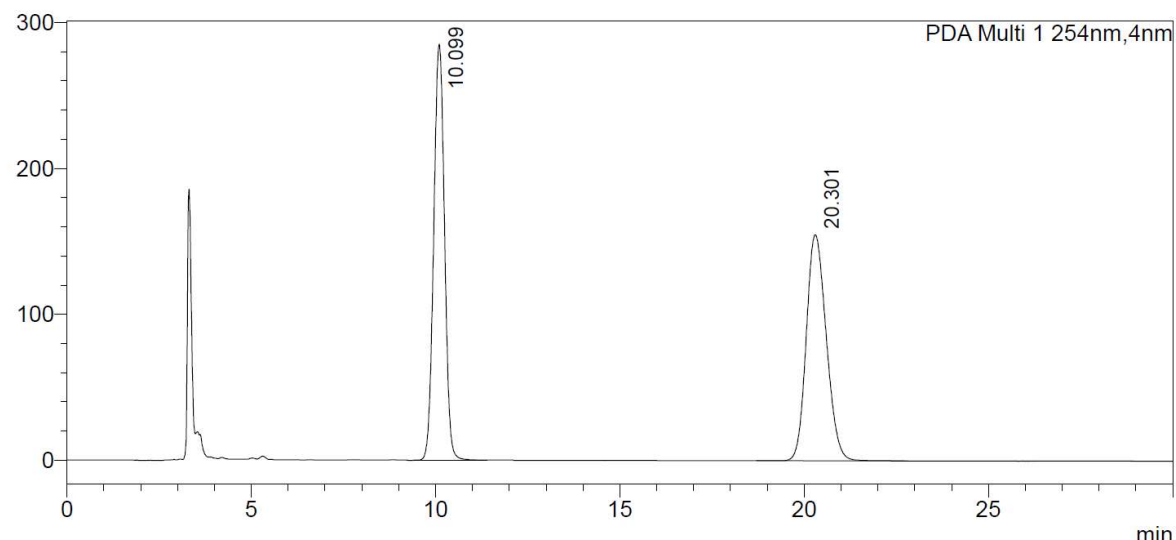

<Peak Table>

PDA Ch1 254nm

\begin{tabular}{|r|r|r|r|r|r|r|r|}
\hline Peak\# & Ret. Time & Area & Height & Conc. & Unit & Mark & Name \\
\hline 1 & 10.099 & 5810694 & 285245 & 0.000 & & M & \\
\hline 2 & 20.301 & 5845938 & 155056 & 0.000 & & $M$ & \\
\hline Total & & 11656632 & 440301 & & & & \\
\hline
\end{tabular}

\section{<Chromatogram>}

mAU

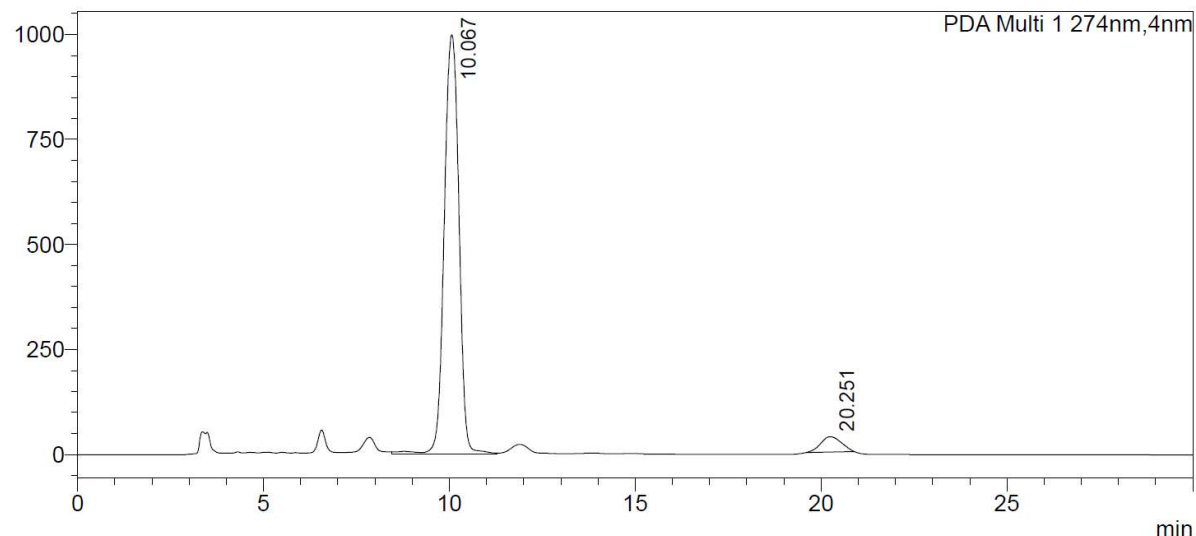

<Peak Table>

PDA Ch1 274nm

\begin{tabular}{|r|r|r|r|r|r|r|r|} 
Peak\# Ret. Time & \multicolumn{1}{|c|}{ Area } & \multicolumn{1}{|c|}{ Height } & \multicolumn{1}{|c|}{ Conc. } & Unit & Mark & Name \\
\hline 1 & 10.067 & 27734332 & 999113 & 95.123 & & $\mathrm{M}$ & \\
\hline 2 & 20.251 & 1422079 & 36574 & 4.877 & & $\mathrm{M}$ & \\
\hline Total & & 29156412 & 1035687 & & & & \\
\hline
\end{tabular}


(1R,2R,4'R)-4'-methyl-2,4'-diphenyl-1'-tosylspiro[indene-1,3'-pyrrolidine]-

\section{$3,5^{\prime}(2 H)$-dione $(3 u)$}

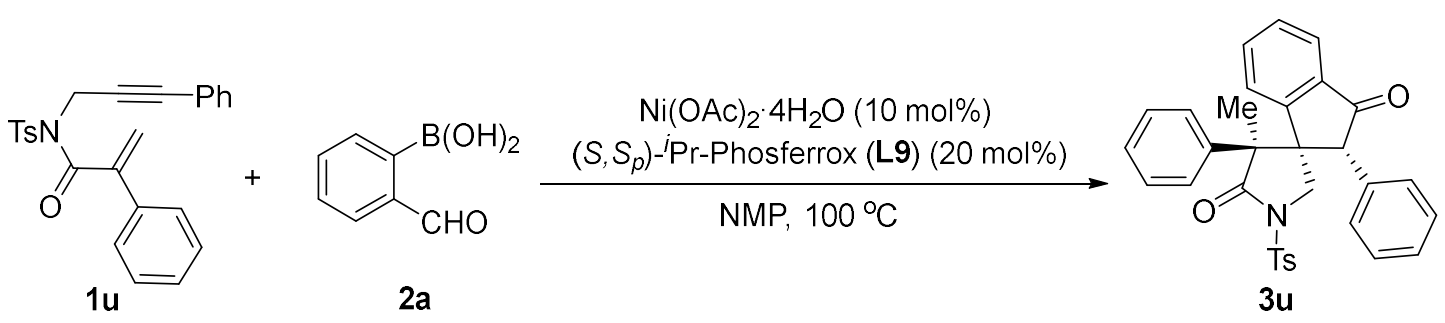

$3 \mathbf{u}$ was prepared according to general procedure using $1 \mathbf{u}(0.1 \mathrm{mmol}, 40.1 \mathrm{mg})$ and

2a $(0.3 \mathrm{mmol}, 45.0 \mathrm{mg})$ and was purified by silica gel column chromatography (petroleum ether/ethyl acetate $=10 / 1 \sim 5 / 1)$ to obtain $3 \mathbf{u}(41.7 \mathrm{mg}, 80 \%$ yield $)$.

${ }^{1} \mathrm{H}$ NMR $\left(600 \mathrm{MHz}, \mathrm{CDCl}_{3}\right)$ ס 7.99-7.90 (m, 1H), 7.58-7.52 (m, 1H), 7.45-7.37 (m, 3H), 7.36-7.33 (m, 1H), 7.32-7.26 (m, 3H), 7.11-7.06 (m, 1H), 7.04-6.94 (m, 3H), 6.87-6.78 (m, 3H), 6.77-6.70 (m, 2H), $4.14(\mathrm{~s}, 1 \mathrm{H}), 3.96(\mathrm{~d}, J=10.7 \mathrm{~Hz}, 1 \mathrm{H}), 3.57(\mathrm{~d}, J=10.8$ $\mathrm{Hz}, 1 \mathrm{H}), 2.50$ (s, 3H), $1.56(\mathrm{~s}, 3 \mathrm{H})$;

${ }^{13} \mathrm{C} \mathrm{NMR}\left(151 \mathrm{MHz}, \mathrm{CDCl}_{3}\right) \delta$ 202.8, 174.8, 154.7, 145.7, 136.9, 136.72, 136.68, 134.9, $134.5,129.8,129.34,129.25,128.6,128.4,128.2$, 128.0, 127.4, 127.1, 125.4, 124.0, 59.2, 59.0, 55.7, 51.1, 21.8, 20.3;

HRMS: (ESI) calcd for $\mathrm{C}_{32} \mathrm{H}_{28} \mathrm{NO}_{4} \mathrm{~S}^{+}[\mathrm{M}+\mathrm{H}]^{+}$522.1734; found 522.1732.

The enantiomeric purity was established by HPLC analysis using a chiral column: OD$\mathrm{H}$ column, $30^{\circ} \mathrm{C}, n$-Hexane/i-Propanol $=80 / 20$ as eluent, $254 \mathrm{~nm}, 1 \mathrm{~mL} / \mathrm{min} . \mathrm{tR}=11$ $\min$ (minor), $17 \mathrm{~min}$ (major).

Optical Rotation: $[\alpha]_{D}^{28}-20.9$ (c 5.0, MeCN) for $97 \%$ ee.

Absolute stereochemistry was determined through X-ray crystallographic analysis. 


\section{<Chromatogram>}

mAU

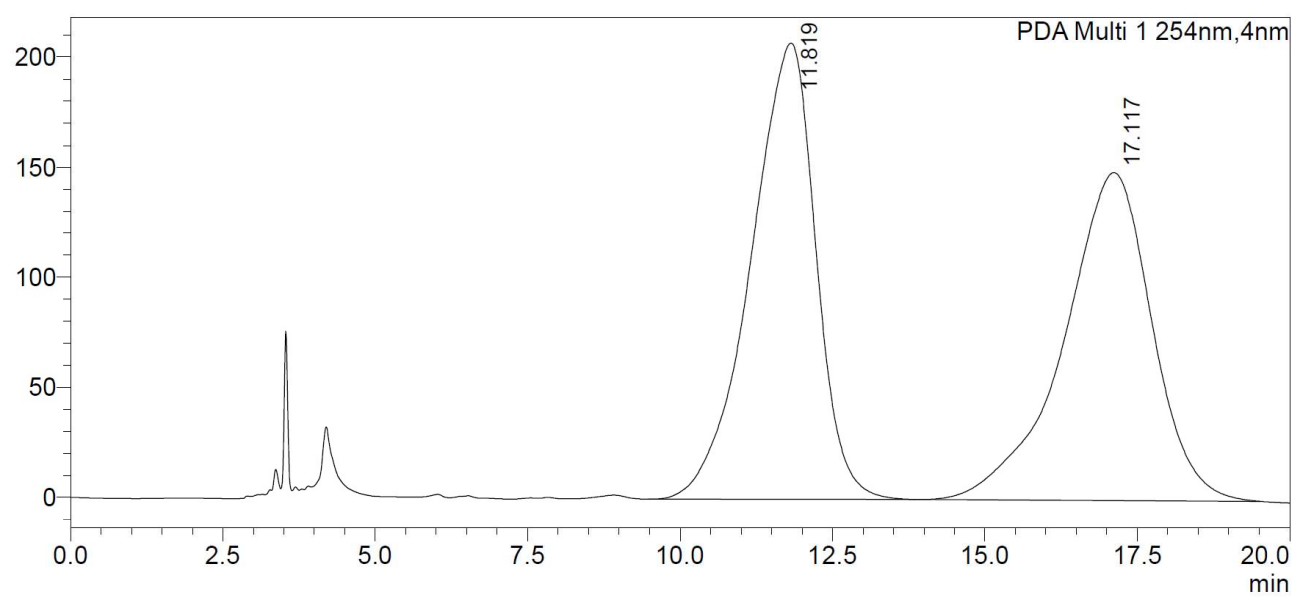

<Peak Table>

PDA Ch1 254nm

Peak\# Ret. Time

\begin{tabular}{|r|r|r|r|r|r|r|r|}
\hline Peak\# & Ret. Time & Area & Height & Conc. & Unit & Mark & Name \\
\hline 1 & 11.819 & 15352900 & 207131 & 0.000 & & $\mathrm{M}$ & \\
\hline 2 & 17.117 & 14971037 & 149109 & 0.000 & & $\mathrm{M}$ & \\
\hline Total & & 30323937 & 356240 & & & & \\
\hline
\end{tabular}

\section{<Chromatogram>}

mAU

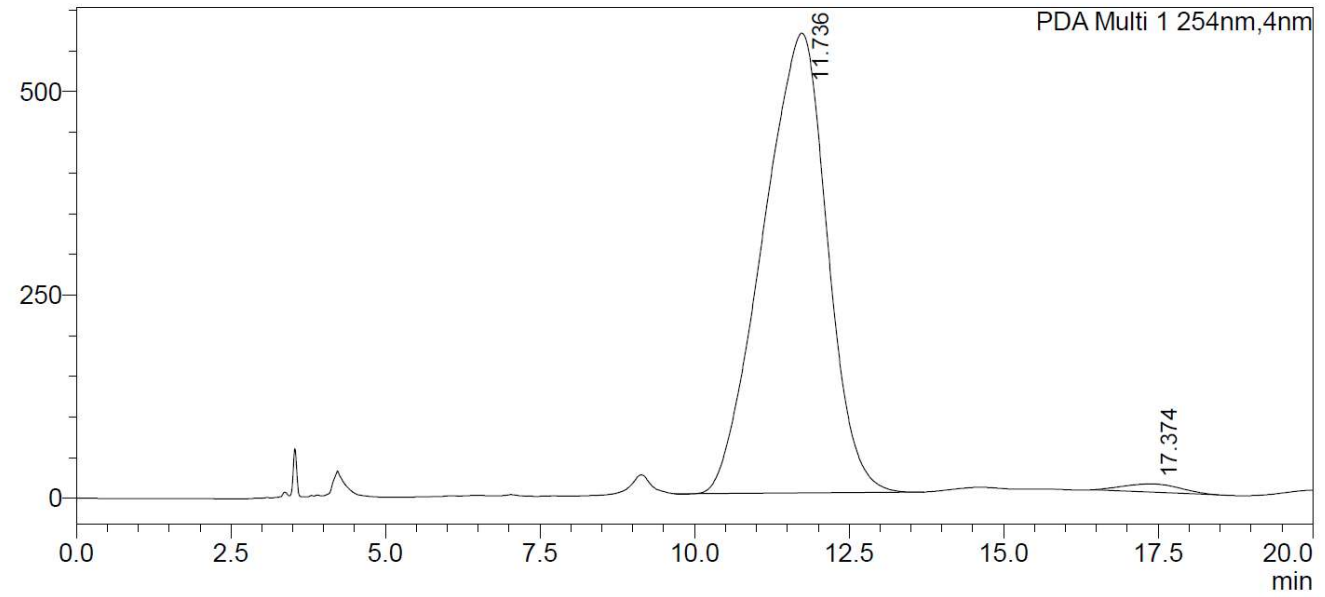

<Peak Table>

PDA Ch1 254nm

\begin{tabular}{|r|r|r|r|r|r|r|r|}
\hline Peak\# Ret. Time & \multicolumn{1}{|c|}{ Area } & Height & Conc. & Unit & Mark & Name \\
\hline 1 & 11.736 & 40928038 & 565643 & 0.000 & & $\mathrm{M}$ & \\
\hline 2 & 17.374 & 652333 & 10183 & 0.000 & & $\mathrm{M}$ & \\
\hline Total & & 41580372 & 575826 & & & & \\
\hline
\end{tabular}


$\left(1 R, 2 R, 4^{\prime} R\right)-4$ '-(4-methoxyphenyl)-4'-methyl-2-phenyl-1'-tosylspiro[indene-1,3'pyrrolidin]-3(2H)-one (3v)

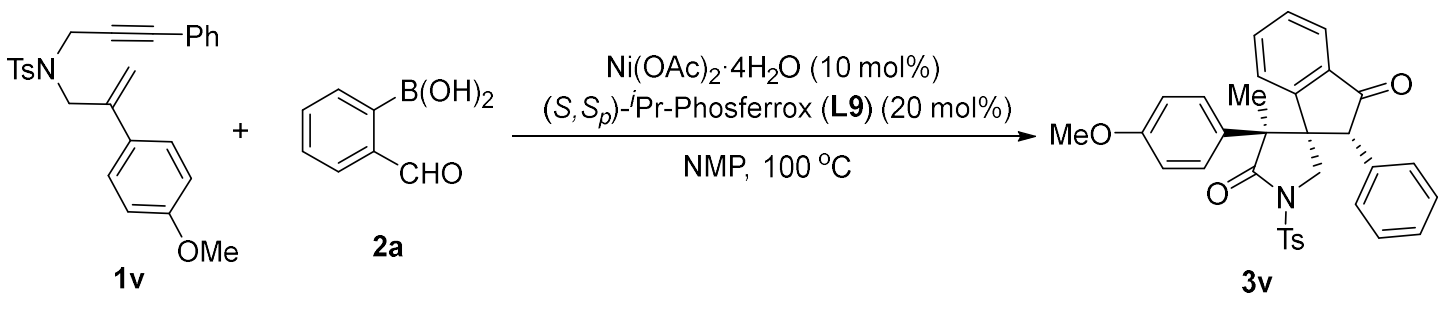

$\mathbf{3} \mathbf{v}$ was prepared according to general procedure using $1 \mathbf{v}(0.1 \mathrm{mmol}, 43.1 \mathrm{mg})$ and $2 \mathbf{a}$ $(0.3 \mathrm{mmol}, 45.0 \mathrm{mg}$ ) and was purified by silica gel column chromatography (petroleum ether/ethyl acetate $=10 / 1 \sim 5 / 1)$ to obtain $3 \mathbf{v}(45.2 \mathrm{mg}, 82 \%$ yield $)$.

${ }^{1} \mathrm{H}$ NMR $\left(600 \mathrm{MHz}, \mathrm{CDCl}_{3}\right)$ ठ 7.96-7.91 (m, 2H), 7.59-7.54 (m, 1H), 7.45-7.41 (m, 1H), 7.40-7.37 (m, 2H), 7.36-7.32 (m, 1H), 7.31-7.25 (m, 3H), 7.10 (d, J = 7.8 Hz, 1H), 6.82 (d, J = 7.2 Hz, 2H), 6.69-6.62 (m, 2H), 6.53-6.48 (m, 2H), $4.09(\mathrm{~s}, 1 \mathrm{H}), 3.96(\mathrm{~d}, J=10.8$ $\mathrm{Hz}, 1 \mathrm{H}), 3.65$ (s, 3H), 3.56 (d, J = $10.8 \mathrm{~Hz}, 1 \mathrm{H}), 2.49$ (s, 3H), 1.52 (s, 3H); ${ }^{13} \mathrm{C}$ NMR $\left(151 \mathrm{MHz}, \mathrm{CDCl}_{3}\right) \delta 202.9,175.0,158.5,154.9,145.6,136.7,136.7,134.9$, $134.6,129.8,129.3,129.2,128.8,128.5,128.3,128.3,128.1,125.3,124.0,113.2$, $59.2,58.5,55.8,55.1,51.1,21.8,20.4$

HRMS: (ESI) calcd for $\mathrm{C}_{33} \mathrm{H}_{29} \mathrm{NO}_{5} \mathrm{~S}^{+}[\mathrm{M}+\mathrm{H}]^{+}$552.1839; found 552.1825.

The enantiomeric purity was established by HPLC analysis using a chiral column: IF column, $30^{\circ} \mathrm{C}, n$-Hexane/i-Propanol $=60 / 40$ as eluent, $254 \mathrm{~nm}, 1 \mathrm{~mL} / \mathrm{min}$. $\mathrm{tR}=10 \mathrm{~min}$ (minor), 21 min (major).

Optical Rotation: $[\alpha]_{D}^{28}-24.8$ (c 5.0, MeCN) for $92 \%$ ee. Absolute stereochemistry was determined through analogy with $\mathbf{3 u}$. 
<Chromatogram>

mAU

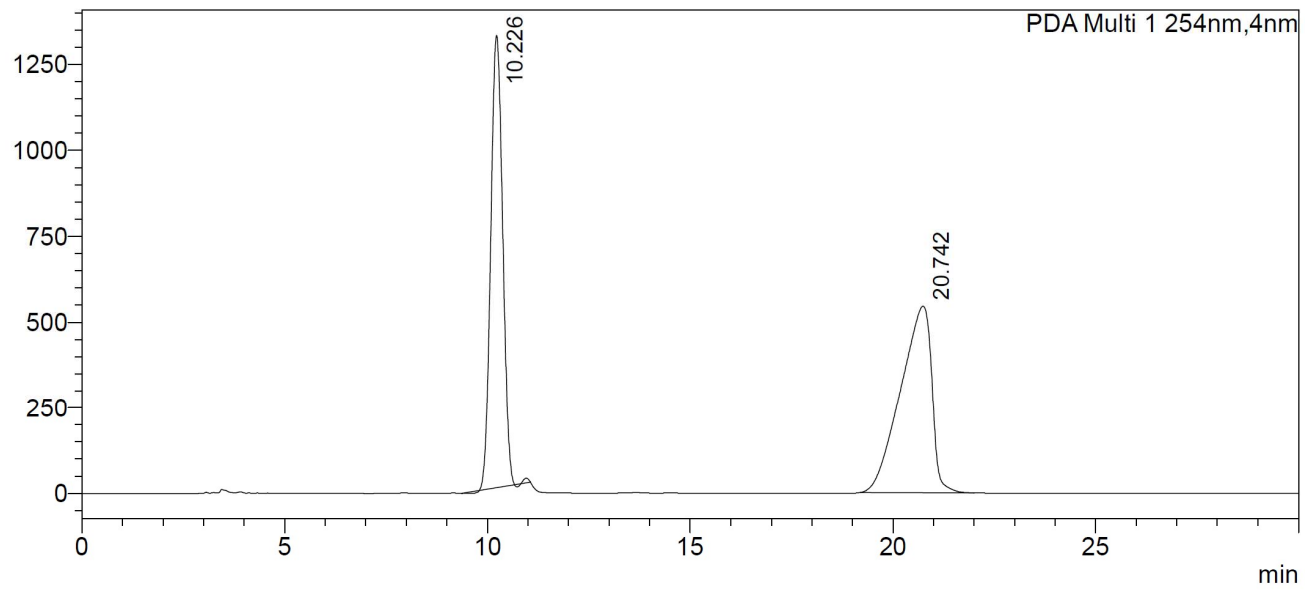

<Peak Table>

PDA Ch1 254nm

\begin{tabular}{|r|r|r|r|r|r|r|r|}
\hline Peak\# Ret. Time & Area & Height & Conc. & Unit & Mark & Name \\
\hline 1 & 10.226 & 27013025 & 1317352 & 0.000 & & $\mathrm{M}$ & \\
\hline 2 & 20.742 & 29320207 & 545311 & 0.000 & & $\mathrm{M}$ & \\
\hline Total & & 56333232 & 1862663 & & & & \\
\hline
\end{tabular}

<Chromatogram>

mAU

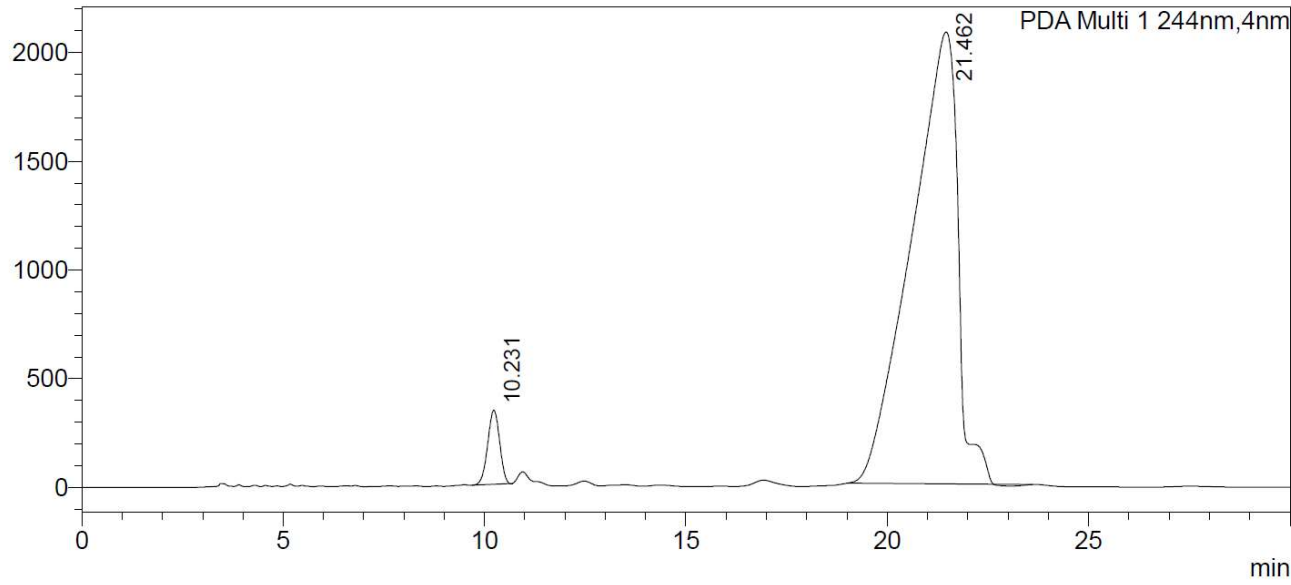

<Peak Table>

PDA Ch1 244nm

\begin{tabular}{|r|r|r|r|r|r|r|r|}
\hline Peak\# Ret. Time & \multicolumn{1}{|c|}{ Area } & \multicolumn{1}{c|}{ Height } & \multicolumn{1}{|c|}{ Conc. } & Unit & Mark & Name \\
\hline 1 & 10.231 & 7068385 & 341284 & 0.000 & & $M$ & \\
\hline 2 & 21.462 & 166791340 & 2079287 & 0.000 & & $M$ & \\
\hline Total & & 173859725 & 2420571 & & & & \\
\hline
\end{tabular}


$\left(1 R, 2 R, 4^{\prime} R\right)-4$ '-(4-fluorophenyl)-4'-methyl-2-phenyl-1'-tosylspiro[indene-1,3'pyrrolidin]-3(2H)-one (3w)

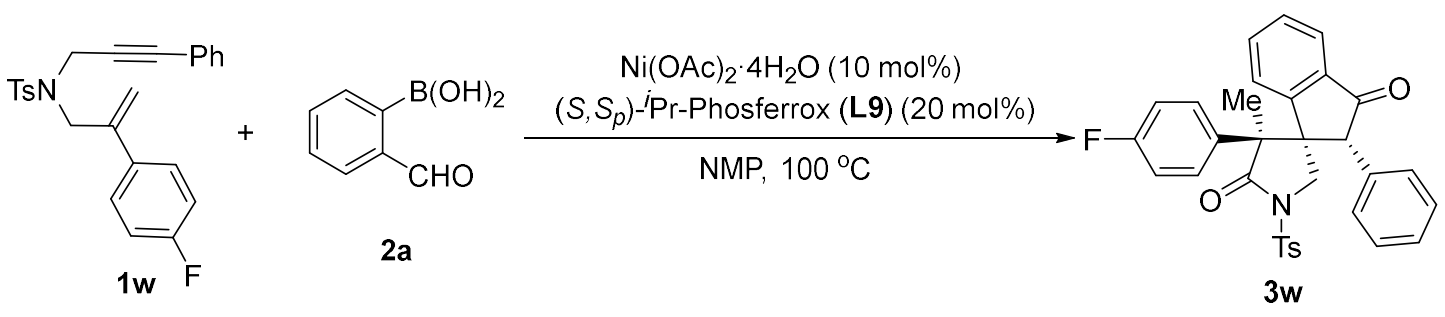

3w was prepared according to general procedure using $1 \mathbf{w}(0.1 \mathrm{mmol}, 41.9 \mathrm{mg})$ and 2a $(0.3 \mathrm{mmol}, 45.0 \mathrm{mg}$ ) and was purified by silica gel column chromatography (petroleum ether/ethyl acetate $=10 / 1 \sim 5 / 1)$ to obtain $3 w(47.4 \mathrm{mg}, 88 \%$ yield).

${ }^{1} \mathrm{H}$ NMR $\left(600 \mathrm{MHz}, \mathrm{CDCl}_{3}\right)$ ס 8.01-7.89 (m, 2H), 7.57-7.53 (m, 1H), 7.48-7.43 (m, 1H), 7.41-7.38 (m, 2H), 7.37-7.33 (m, 1H), 7.32-7.27 (m, 3H), 7.20-7.15 (m, 1H), 6.86-6.82 (m, 2H), 6.7-6.74 (m, 2H), 6.70-6.64 (m, 2H), $4.10(\mathrm{~s}, 1 \mathrm{H}), 3.96(\mathrm{~d}, J=10.8 \mathrm{~Hz}, 1 \mathrm{H})$, $3.56(\mathrm{~d}, J=10.8 \mathrm{~Hz}, 1 \mathrm{H}), 2.49$ (s, 3H), 1.55 (s, 3H);

${ }^{13} \mathrm{C}$ NMR (151 MHz, $\mathrm{CDCl}_{3}$ ) $\delta$ 202.6, 174.6, 161.6 (d, $\left.J=248.3 \mathrm{~Hz}\right), 154.9,145.8$, 136.53, 136.45, 135.2, 134.4, 132.6 (d, J = 3.4 Hz), 129.8, 129.38, 129.35, 128.9 (d, J $=8.1 \mathrm{~Hz}), 128.5,128.30,128.24,125.0,124.1,114.8(\mathrm{~d}, J=21.3 \mathrm{~Hz}), 58.9,58.5,55.5$, 51.2, 21.8, 20.7;

${ }^{19} \mathrm{~F} \mathrm{NMR}\left(376 \mathrm{MHz}, \mathrm{CDCl}_{3}\right) \delta-114.2(\mathrm{~m})$;

HRMS: (ESI) calcd for $\mathrm{C}_{32} \mathrm{H}_{27} \mathrm{NFO}_{4} \mathrm{~S}^{+}[\mathrm{M}+\mathrm{H}]^{+}$540.1639; found 540.1637.

The enantiomeric purity was established by HPLC analysis using a chiral column: OD$\mathrm{H}$ column, $30^{\circ} \mathrm{C}, n$-Hexane/i-Propanol $=90 / 10$ as eluent, $254 \mathrm{~nm}, 1 \mathrm{~mL} / \mathrm{min}$. $\mathrm{tR}=20$ $\min$ (minor), $23 \mathrm{~min}$ (major).

Optical Rotation: $[\alpha]_{D}^{28}-20.8$ (c 5.0, MeCN) for $92 \%$ ee.

Absolute stereochemistry was determined through analogy with $3 \mathbf{u}$. 
<Chromatogram>

mAU

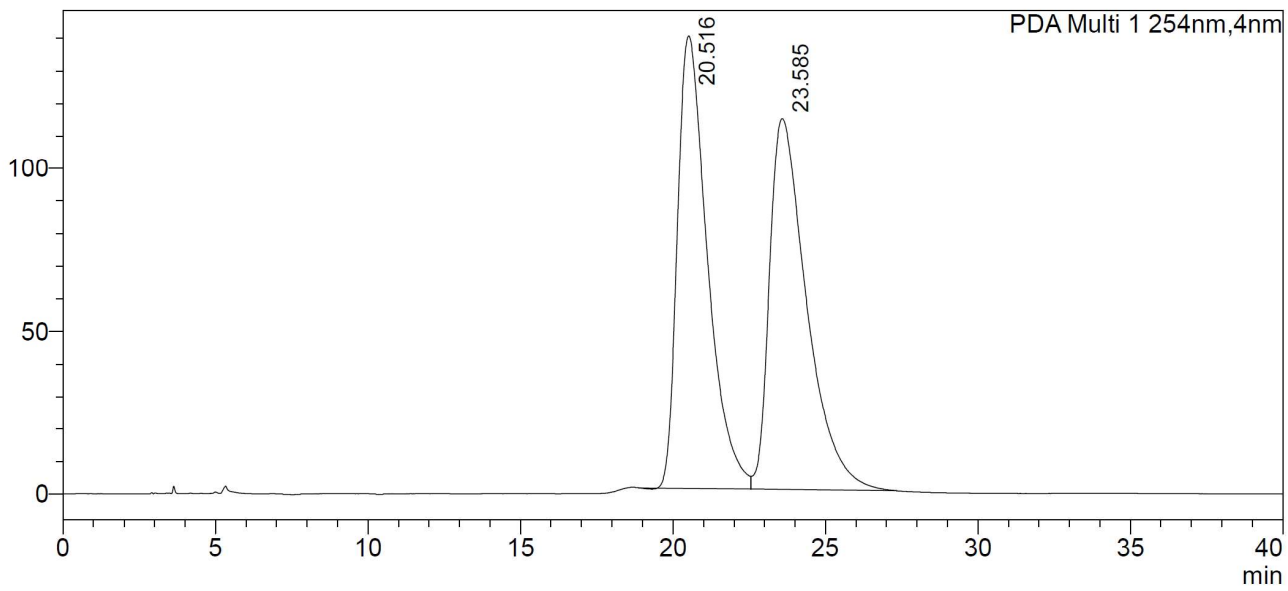

<Peak Table>

PDA Ch1 254nm

\begin{tabular}{|c|c|c|c|c|c|c|c|}
\hline Peak\# & Ret. Time & Area & Height & Conc. & Unit & Mark & Name \\
\hline 1 & 20.516 & 9524304 & 138973 & 0.000 & & M & \\
\hline 2 & 23.585 & 9692908 & 114025 & 0.000 & & V M & \\
\hline Total & & 19217213 & 252998 & & & & \\
\hline
\end{tabular}

<Chromatogram>

mAU

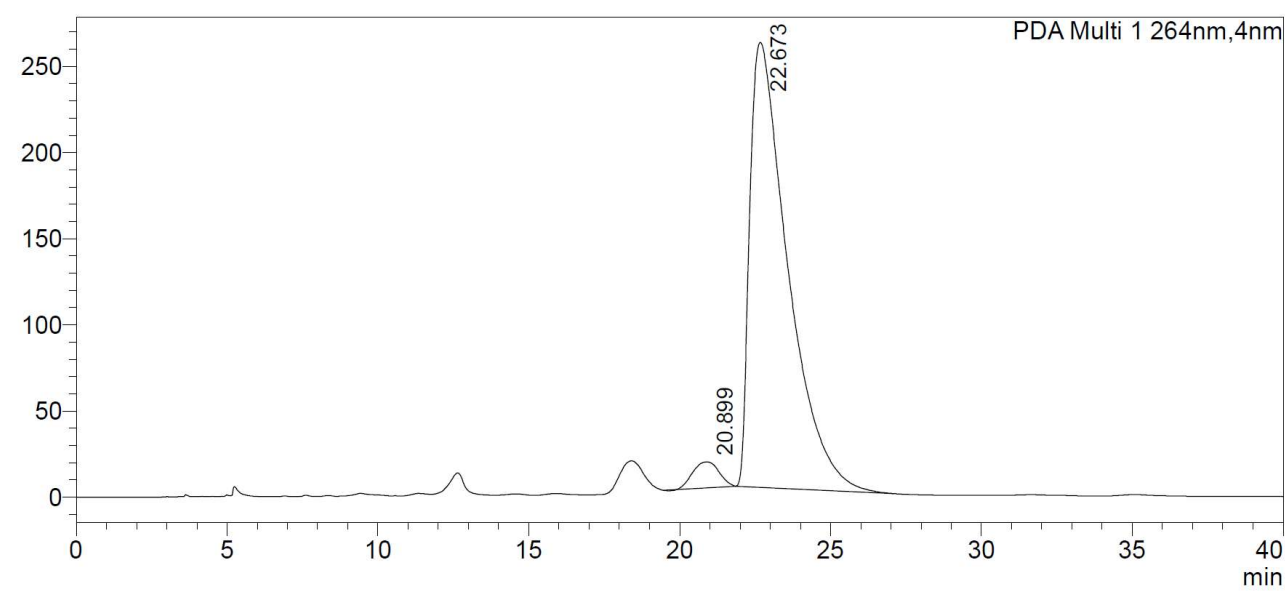

<Peak Table>

PDA Ch1 264nm

\begin{tabular}{|r|r|r|r|r|r|r|r|}
\hline Peak\# Ret. Time & \multicolumn{1}{|c|}{ Area } & Height & Conc. & Unit & Mark & Name \\
\hline 1 & 20.899 & 907554 & 15103 & 0.000 & & M & \\
\hline 2 & 22.673 & 22626306 & 258181 & 0.000 & & $M$ & \\
\hline Total & & 23533860 & 273284 & & & &
\end{tabular}


(1R,2R,4'R)-4'--methyl-4'-(naphthalen-2-yl)-2-phenyl-1'-tosylspiro[indene-1,3'pyrrolidine]-3,5'(2H)-dione (3x)

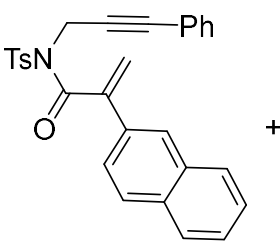

$1 \mathrm{x}$

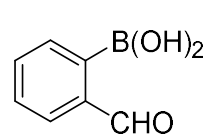

$2 a$

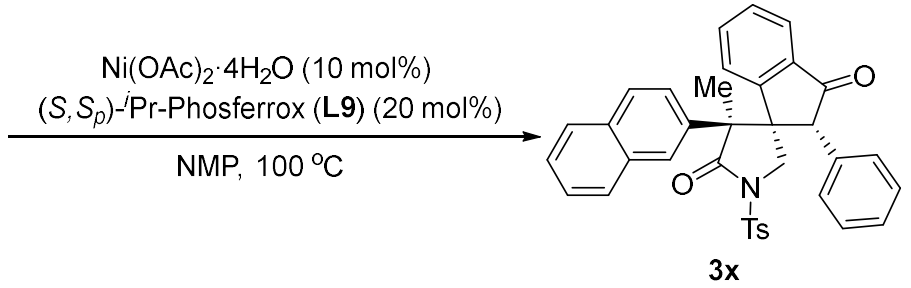

$3 \mathbf{x}$ was prepared according to general procedure using $1 \mathbf{x}(0.1 \mathrm{mmol}, 46.5 \mathrm{mg})$ and $2 \mathbf{a}$ $(0.3 \mathrm{mmol}, 45.0 \mathrm{mg}$ ) and was purified by silica gel column chromatography (petroleum ether/ethyl acetate $=10 / 1 \sim 5 / 1)$ to obtain $3 \times(52.0 \mathrm{mg}, 91 \%$ yield $)$.

${ }^{1} \mathrm{H}$ NMR $\left(600 \mathrm{MHz}, \mathrm{CDCl}_{3}\right)$ ठ 8.01-7.96 (m, 2H), 7.67-7.62 (m, 1H), 7.54-7.50 (m, 1H), 7.49-7.45 (m, 1H), 7.44-7.40 (m, 3H), 7.39-7.33 (m, 3H), 7.32-7.26 (m, 4H), 7.24 (d, J $=2.0 \mathrm{~Hz}, 1 \mathrm{H}), 7.05-6.99(\mathrm{~m}, 1 \mathrm{H}), 6.89-6.80(\mathrm{~m}, 2 \mathrm{H}), 6.71-6.66(\mathrm{~m}, 1 \mathrm{H}), 4.24(\mathrm{~s}, 1 \mathrm{H})$, 4.00 (d, $J=10.7 \mathrm{~Hz}, 1 \mathrm{H}), 3.62(\mathrm{~d}, J=10.7 \mathrm{~Hz}, 1 \mathrm{H}), 2.53(\mathrm{~s}, 3 \mathrm{H}), 1.64(\mathrm{~s}, 3 \mathrm{H})$; ${ }^{13} \mathrm{C}$ NMR $\left(151 \mathrm{MHz}, \mathrm{CDCl}_{3}\right) \delta 202.7,174.9,154.0,145.7,136.9,136.7,134.7,134.6$, 134.6, 132.4, 132.0, 129.9, 129.3, 128.6, 128.4, 128.2, 128.0, 127.4, 127.2, 126.4, $126.3,126.1,125.7,124.8,124.0,59.6,59.3,56.0,50.93,2.78,20.3$; HRMS: (ESI) calcd for $\mathrm{C}_{36} \mathrm{H}_{30} \mathrm{NO}_{4} \mathrm{~S}^{+}[\mathrm{M}+\mathrm{H}]^{+}$572.1890; found 572.1893 The enantiomeric purity was established by HPLC analysis using a chiral column: OD$\mathrm{H}$ column, $30^{\circ} \mathrm{C}, n$-Hexane/i-Propanol $=80 / 20$ as eluent, $254 \mathrm{~nm}, 1 \mathrm{~mL} / \mathrm{min} . \mathrm{tR}=11$ $\min$ (major), 14 min (minor).

Optical Rotation: $[\alpha]_{D}^{28}-28.5$ (c 5.0, MeCN) for $90 \%$ ee. Absolute stereochemistry was determined through analogy with $\mathbf{3 u}$. 
$<$ Chromatogram>

mAU

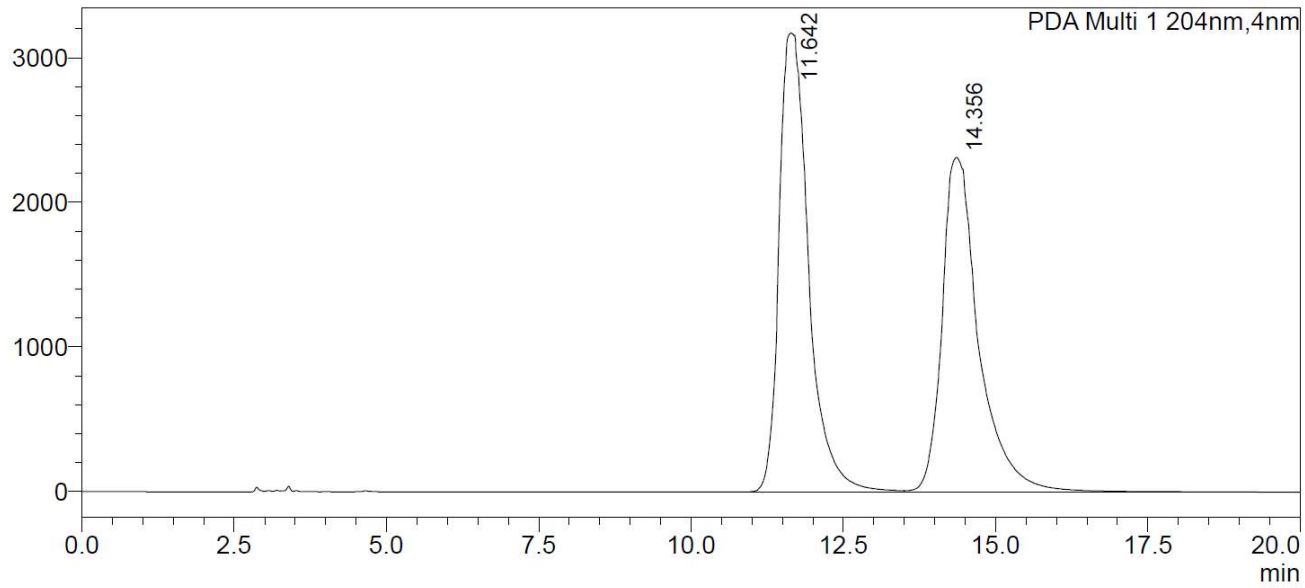

<Peak Table>

PDA Ch1 204nm

\begin{tabular}{|r|r|r|r|r|r|r|r|}
\hline Peak\# Ret. Time & \multicolumn{1}{|c|}{ Area } & Height & Conc. & Unit & Mark & Name \\
\hline 1 & 11.642 & 106947224 & 3172130 & 0.000 & & & \\
\hline 2 & 14.356 & 97039027 & 2314819 & 0.000 & & V & \\
\hline Total & & 203986251 & 5486949 & & & &
\end{tabular}

\section{$<$ Chromatogram $>$}

mAU

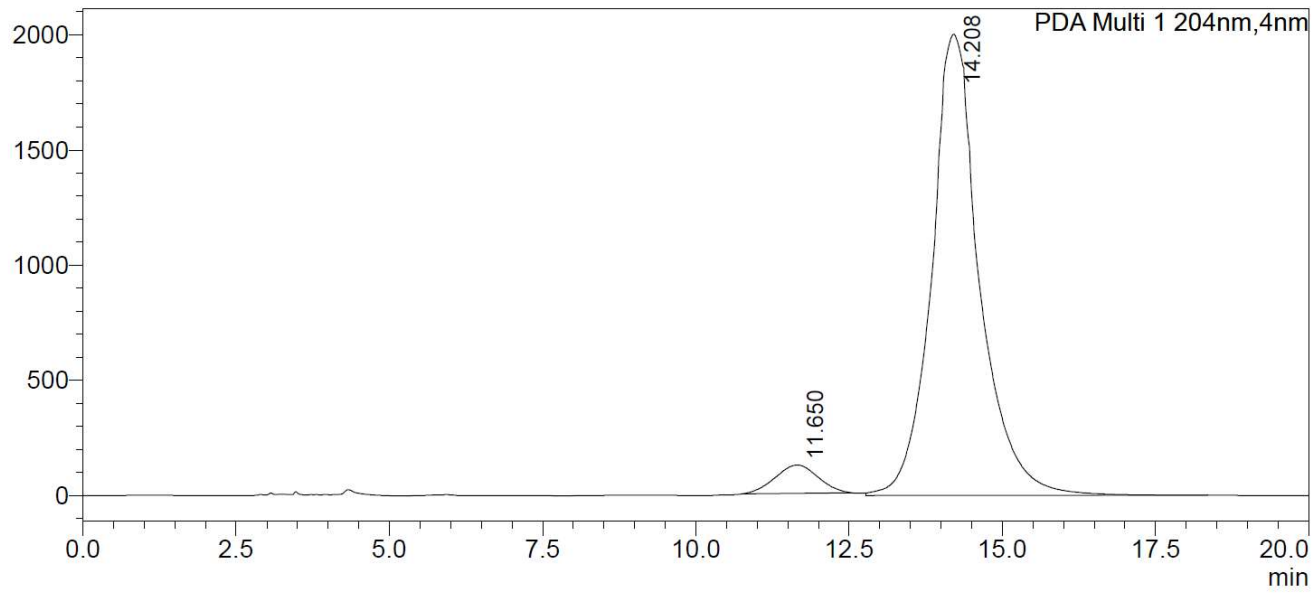

<Peak Table>

PDA Ch1 204nm

Peak\# Ret. Tim

\begin{tabular}{|r|r|}
\hline 1 & 11.650 \\
\hline 2 & 14.208 \\
\hline
\end{tabular}

Area

5970088

Height

Conc.

0.000
0.000

Name

\begin{tabular}{|r|r|r|r|}
\hline Total & 14.208 & 108884188 & 2002253 \\
\hline
\end{tabular} 
$(1 R, 2 R, 4$ 'R)-4'-ethyl-2-phenyl-1'-tosylspiro[indene-1,3'-pyrrolidine]-3,5'(2H)dione (3y)<smiles>C/C=C/C(=O)N([13CH3])CC#Cc1ccccc1</smiles>

$1 y$<smiles>COc1ccccc1C=O</smiles>

$2 a$

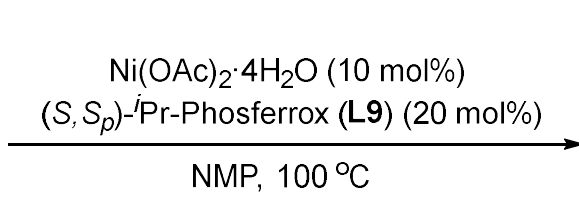

3y

3y was prepared according to general procedure using $1 \mathbf{y}(0.1 \mathrm{mmol}, 35.3 \mathrm{mg})$ and $\mathbf{2 a}$ $(0.3 \mathrm{mmol}, 45.0 \mathrm{mg}$ ) and was purified by silica gel column chromatography (petroleum ether/ethyl acetate $=10 / 1 \sim 5 / 1)$ to obtain $3 y(15.6 \mathrm{mg}, 34 \%$ yield $)$.

${ }^{1} \mathrm{H}$ NMR $\left(600 \mathrm{MHz}, \mathrm{CDCl}_{3}\right)$ ठ 7.90-7.86 (m, 1H), 7.75-7.70 (m, 1H), 7.66-7.61 (m, 2H), 7.60-7.53 (m, 2H), 7.31-7.26 (m, 2H), 7.25-7.18 (m, 3H), 6.91-6.84 (m, 2H), $3.95(\mathrm{~s}$, 1H), $3.87(\mathrm{~d}, J=10.7 \mathrm{~Hz}, 1 \mathrm{H}), 3.81(\mathrm{~d}, J=10.7 \mathrm{~Hz}, 1 \mathrm{H}), 2.88-2.84(\mathrm{~m}, 1 \mathrm{H}), 2.47(\mathrm{~s}$, $3 \mathrm{H}), 1.95-1.90(\mathrm{~m}, 1 \mathrm{H}), 1.51-1.45(\mathrm{~m}, 1 \mathrm{H}), 0.80(\mathrm{t}, J=7.5 \mathrm{~Hz}, 3 \mathrm{H})$; ${ }^{13} \mathrm{C}$ NMR $\left(151 \mathrm{MHz}, \mathrm{CDCl}_{3}\right) \delta 203.3,172.6,145.0,136.6,136.1,135.4,135.0,129.6$, 129.6, 129.5, 129.1, 128.2, 127.9, 124.5, 123.6, 57.8, 55.8, 53.0, 51.1, 21.7, 18.9, 12.5; HRMS: (ESI) calcd for $\mathrm{C}_{27} \mathrm{H}_{26} \mathrm{NO}_{4} \mathrm{~S}^{+}[\mathrm{M}+\mathrm{H}]^{+} 460.1577$; found 460.1576 .

The enantiomeric purity was established by HPLC analysis using a chiral column: OD$\mathrm{H}$ column, $30^{\circ} \mathrm{C}, n$-Hexane/i-Propanol $=80 / 20$ as eluent, $254 \mathrm{~nm}, 1 \mathrm{~mL} / \mathrm{min} . \mathrm{tR}=17$ $\min$ (major), 22 min (minor).

Optical Rotation: $[\alpha]_{D}^{28}-29.6$ (c 5.0, MeCN) for $98 \%$ ee.

Absolute stereochemistry was determined through analogy with $\mathbf{3 u}$. 


\section{<Chromatogram>}

mAU

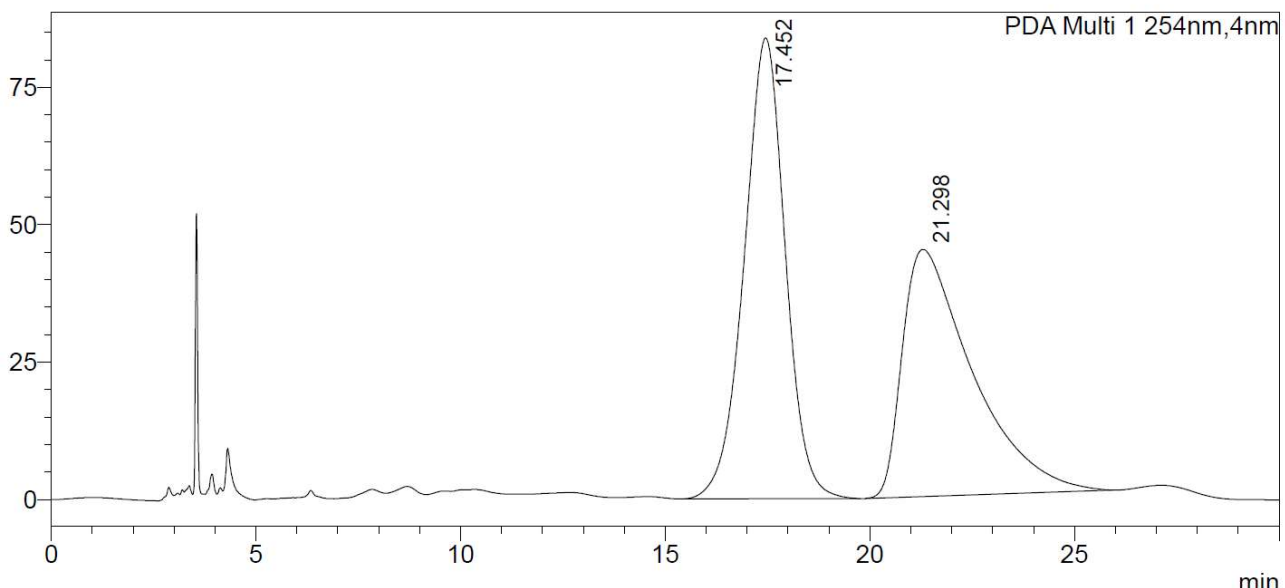

<Peak Table>

PDA Ch1 254nm

\begin{tabular}{|r|r|r|r|r|r|r|r|}
\hline Peak\# Ret. Time & \multicolumn{1}{|c|}{ Area } & Height & Conc. & Unit & Mark & Name \\
\hline 1 & 17.452 & 5848080 & 83780 & 0.000 & & & \\
\hline 2 & 21.298 & 5355373 & 44931 & 0.000 & & & \\
\hline Total & & 11203453 & 128711 & & & & \\
\hline
\end{tabular}

\section{<Chromatogram>}

mAU

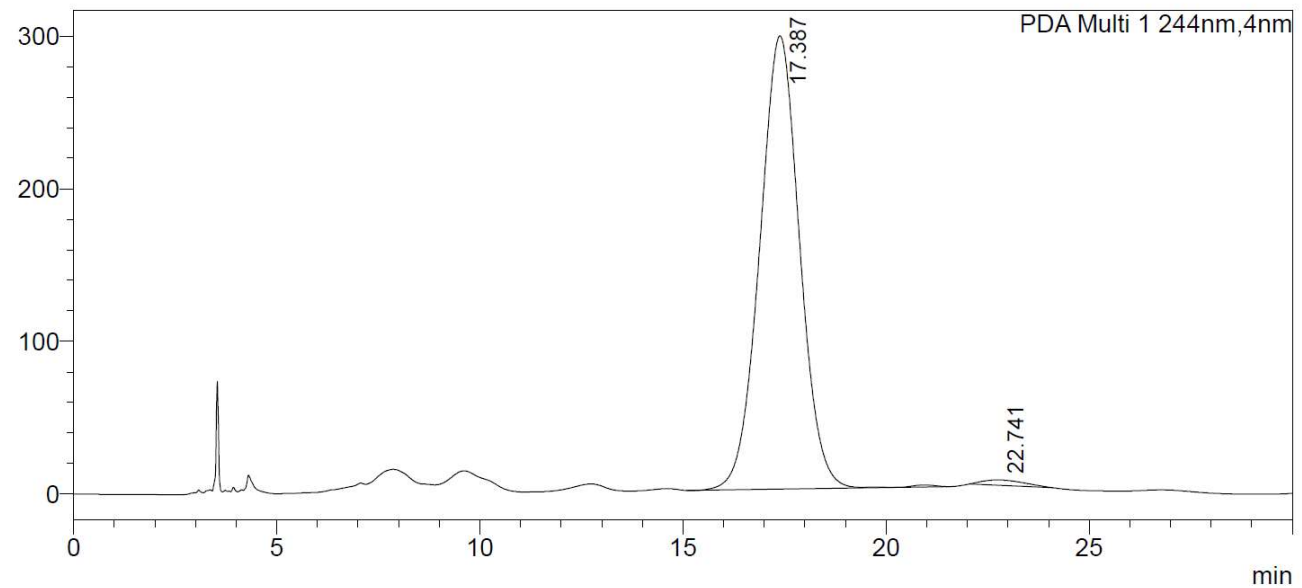

<Peak Table>

PDA Ch1 244nm

Peak\# Ret. Time

\begin{tabular}{|r|r|}
\hline 1 & 17.387 \\
\hline 2 & 22.741 \\
\hline Total & \\
\hline
\end{tabular}

Area

Height $\quad$ Conc.

\begin{tabular}{l|l|l} 
Unit & Mark & Name
\end{tabular}

297086

3469

0.000

\begin{tabular}{r|r|}
20592533 & 300555 \\
\hline
\end{tabular}

\begin{tabular}{|l|l|}
\hline $\mathrm{M}$ & \\
$\mathrm{M}$ & \\
\hline
\end{tabular}


$\left(1 R, 2 R, 4^{\prime} R\right)-4$ '-ethyl-4'-methyl-2-phenyl-1'-tosylspiro[indene-1,3'-pyrrolidine]-

\section{$3,5^{\prime}(2 H)$-dione $(3 z)$}

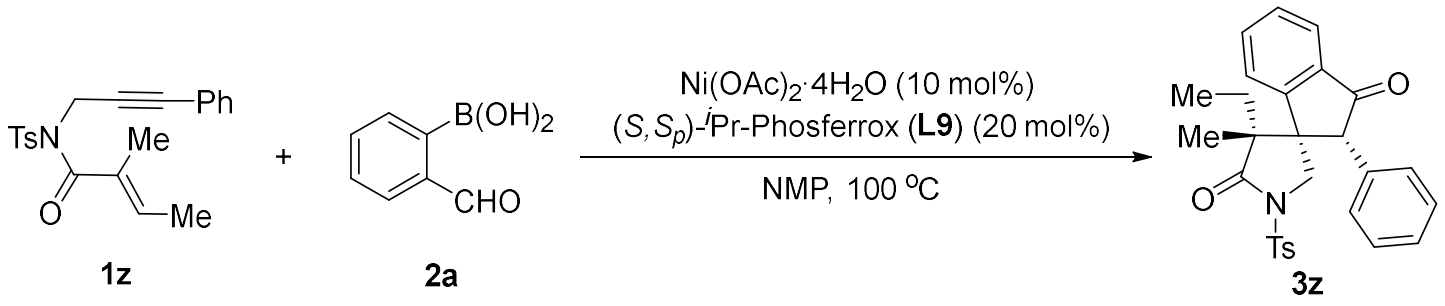

$3 z$ was prepared according to general procedure using $1 \mathbf{z}(0.1 \mathrm{mmol}, 36.7 \mathrm{mg})$ and $\mathbf{2 a}$ $(0.3 \mathrm{mmol}, 45.0 \mathrm{mg})$ and was purified by silica gel column chromatography (petroleum ether/ethyl acetate $=10 / 1 \sim 5 / 1)$ to obtain $3 z(19.9 \mathrm{mg}, 42 \%$ yield $)$.

${ }^{1} \mathrm{H}$ NMR $\left(600 \mathrm{MHz}, \mathrm{CDCl}_{3}\right)$ ס 7.94-7.92 (m, 2H), 7.84-7.81 (m, 1H), 7.49-7.45 (m, 1H), 7.42-7.39 (m, 1H), 7.38-7.36 (m, 2H), 7.35-7.31 (m, 3H), 6.97-6.93 (m, 3H), $3.90(\mathrm{~s}$, 1H), 3.75 (d, J = $10.9 \mathrm{~Hz}, 1 \mathrm{H}), 3.60$ (d, $J=10.9 \mathrm{~Hz}, 1 \mathrm{H}), 2.49(\mathrm{~s}, 3 \mathrm{H}), 1.78-1.74(\mathrm{~m}$, $1 \mathrm{H}), 1.66-1.62(\mathrm{~m}, 1 \mathrm{H}), 0.76(\mathrm{t}, J=7.5 \mathrm{~Hz}, 3 \mathrm{H}), 0.61(\mathrm{~s}, 3 \mathrm{H})$;

${ }^{13} \mathrm{C}$ NMR $\left(151 \mathrm{MHz}, \mathrm{CDCl}_{3}\right) \delta 204.1,175.2,157.2,145.5,137.0,136.2,135.8,135.0$, $129.7,129.3,129.2,128.9,128.3,128.1,124.3,123.8,57.0,54.3,53.3,52.8,28.3$, 21.7, 15.2, 8.9;

HRMS: (ESI) calcd for $\mathrm{C}_{28} \mathrm{H}_{28} \mathrm{NO}_{4} \mathrm{~S}^{+}[\mathrm{M}+\mathrm{H}]^{+}$474.1734; found 474.1731.

The enantiomeric purity was established by HPLC analysis using a chiral column: OD$\mathrm{H}$ column, $30^{\circ} \mathrm{C}, n$-Hexane $/ \mathrm{i}$-Propanol $=80 / 20$ as eluent, $254 \mathrm{~nm}, 1 \mathrm{~mL} / \mathrm{min}$. $\mathrm{tR}=8 \mathrm{~min}$ (major), 17 min (minor).

Optical Rotation: $[\alpha]_{D}^{28}-19.0$ (c 5.0, MeCN) for $97 \%$ ee.

Absolute stereochemistry was determined through analogy with $\mathbf{3 u}$. 
$\mathrm{mAU}$

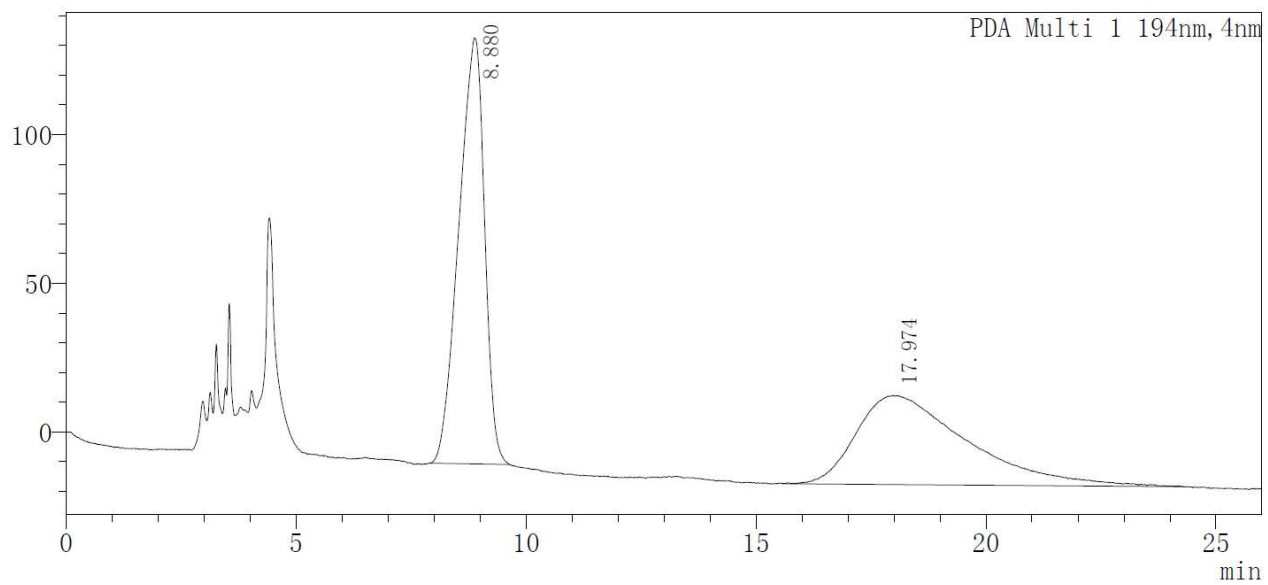

〈峰表〉

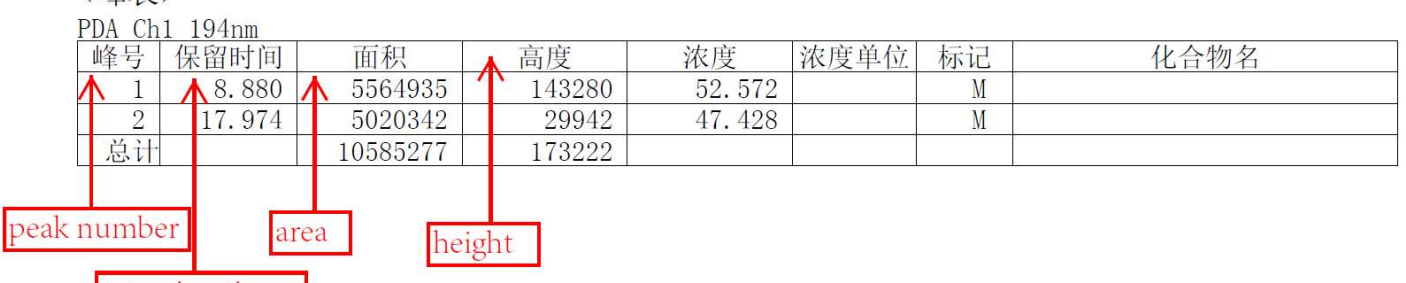

retention time

mAU

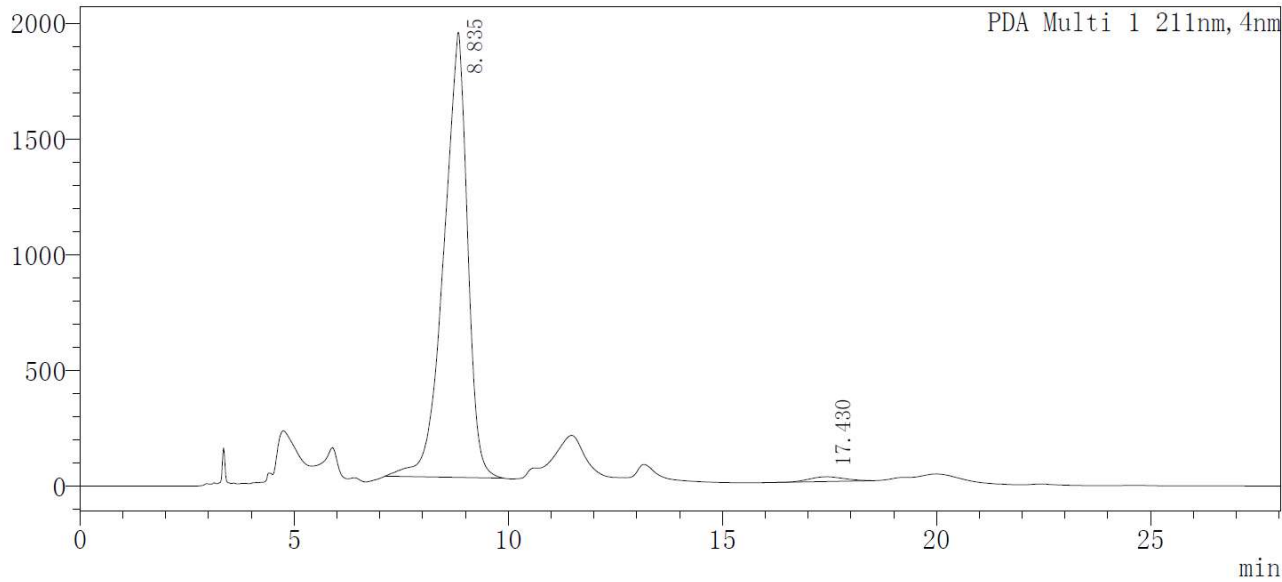

〈峰表〉

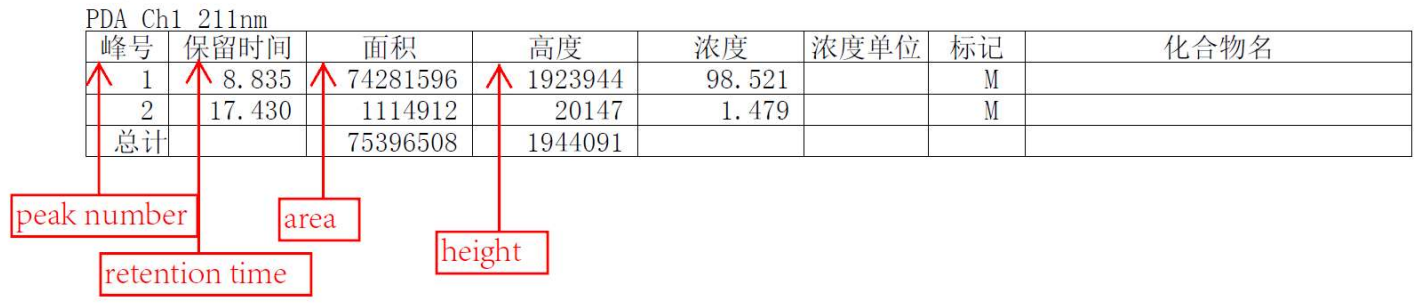




\section{(2"R,4'S)-2"-phenyl-1'-tosyldispiro[cyclododecane-1,3'-pyrrolidine-4',1"-}

\section{indene]-2',3"(2"H)-dione (3aa)}

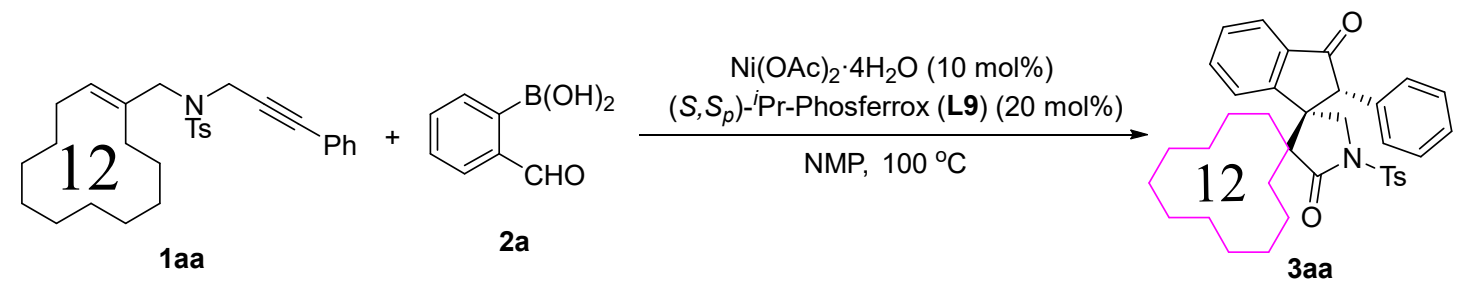

3aa was prepared according to general procedure using 1 aa $(0.1 \mathrm{mmol}, 46.3 \mathrm{mg})$ and 2a $(0.3 \mathrm{mmol}, 45.0 \mathrm{mg})$ and was purified by silica gel column chromatography (petroleum ether/ethyl acetate $=10 / 1 \sim 5 / 1)$ to obtain 3aa $(14.2 \mathrm{mg}, 30 \%$ yield).

${ }^{1} \mathrm{H} \operatorname{NMR}\left(600 \mathrm{MHz}, \mathrm{CDCl}_{3}\right) \delta 7.83(\mathrm{~d}, J=7.5 \mathrm{~Hz}, 1 \mathrm{H}), 7.64-7.61(\mathrm{~m}, 2 \mathrm{H}), 7.60-7.56(\mathrm{~m}$, $1 \mathrm{H}), 7.53-7.47(\mathrm{~m}, 1 \mathrm{H}), 7.36(\mathrm{~d}, J=7.9 \mathrm{~Hz}, 1 \mathrm{H}), 7.31(\mathrm{~d}, J=8.0 \mathrm{~Hz}, 2 \mathrm{H}), 7.27-7.21(\mathrm{~m}$, 3H), $6.80(\mathrm{~s}, 2 \mathrm{H}), 4.01(\mathrm{~s}, 1 \mathrm{H}), 3.51(\mathrm{~d}, J=11.1 \mathrm{~Hz}, 1 \mathrm{H}), 3.24(\mathrm{~d}, J=11.4 \mathrm{~Hz}, 1 \mathrm{H})$, $3.22(\mathrm{~d}, J=10.6 \mathrm{~Hz}, 1 \mathrm{H}), 3.07$ (d, $J=10.5 \mathrm{~Hz}, 1 \mathrm{H}), 2.47(\mathrm{~s}, 3 \mathrm{H}), 1.39-1.03(\mathrm{~m}, 17 \mathrm{H})$, 0.92-0.75 (m, 3H), 0.73-0.63 (m, 1H), 0.53-0.43 (m, 1H);

${ }^{13} \mathrm{C}$ NMR $\left(151 \mathrm{MHz}, \mathrm{CDCl}_{3}\right) \delta 206.0,158.3,143.7,138.6,136.6,135.8,134.2,129.7$, $129.0,128.8,127.7,127.2,126.2,123.7,59.6,58.2,56.3,55.4,50.6,29.7,29.3,28.9$, $26.8,25.9,22.8,22.7,22.6,21.9,21.5,20.4,18.5$;

HRMS: (ESI) calcd for $\mathrm{C}_{28} \mathrm{H}_{28} \mathrm{NO}_{4} \mathrm{~S}^{+}[\mathrm{M}+\mathrm{H}]^{+}$474.1734; found 474.1731.

The enantiomeric purity was established by HPLC analysis using a chiral column: OD$\mathrm{H}$ column, $30^{\circ} \mathrm{C}, n$-Hexane $/ \mathrm{i}$-Propanol $=95 / 5$ as eluent, $254 \mathrm{~nm}, 1 \mathrm{~mL} / \mathrm{min}$. $\mathrm{tR}=10 \mathrm{~min}$ (major), 14 min (minor)

Optical Rotation: $[\alpha]_{D}{ }^{28}-113.8$ (c 5.0, MeCN) for 95\% ee.

Absolute stereochemistry was determined through analogy with $\mathbf{3 u}$. 
$<$ Chromatogram>

mAU

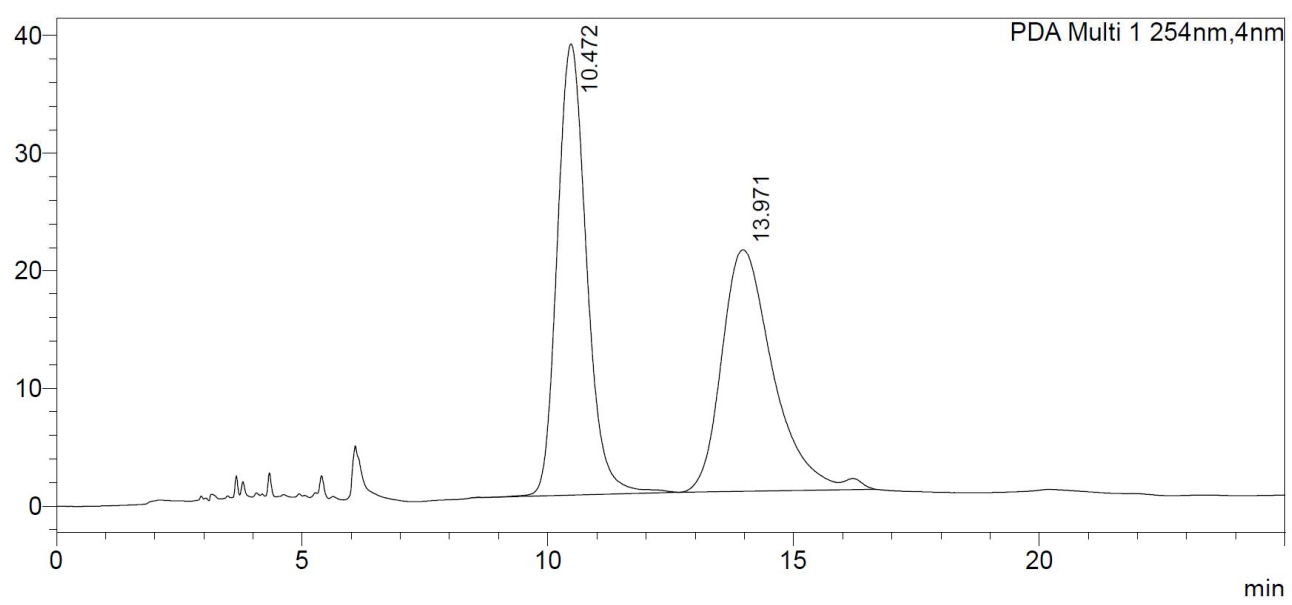

<Peak Table>

PDA Ch1 254nm

\begin{tabular}{rr|l} 
Peak\# Ret. Time & Area \\
\hline 1 & 10.472 & 159064
\end{tabular}

\begin{tabular}{l|r} 
Area & Height \\
\hline 590640 & 38341
\end{tabular}

1492955

\begin{tabular}{|l|r|r|}
\hline Total & 3083596 & 58899
\end{tabular}

38341

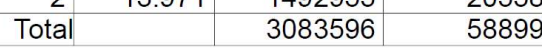

Conc.

\begin{tabular}{c|c|c}
\hline 0.000 & Unit & Mark \\
\hline & & $\mathrm{M}$
\end{tabular}

0.000

$\mathrm{M}$

\section{$<$ Chromatogram>}

mAU

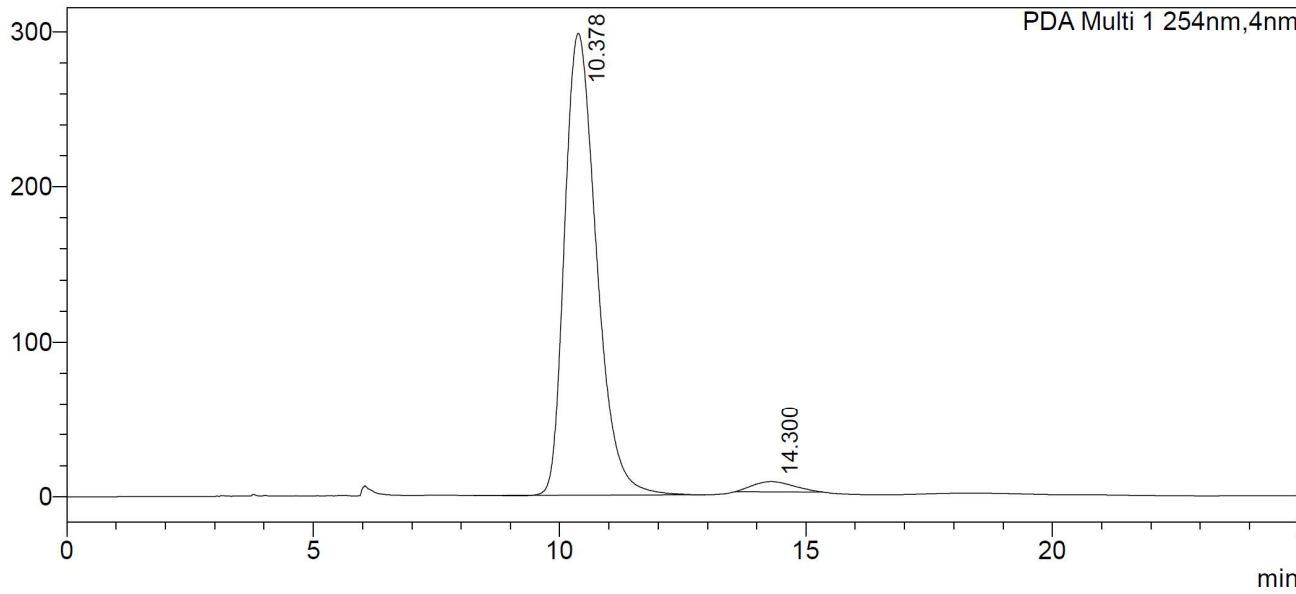

<Peak Table>

PDA Ch1 254nm

\begin{tabular}{|r|r|r|r|r|r|r|r|}
\hline Peak\# & Ret. Time & \multicolumn{1}{|c|}{ Area } & Height & Conc. & Unit & Mark & Name \\
\hline 1 & 10.378 & 13431873 & 297969 & 0.000 & & $\mathrm{M}$ & \\
\hline 2 & 14.300 & 376622 & 6531 & 0.000 & & $\mathrm{M}$ & \\
\hline Total & & 13808496 & 304500 & & & & \\
\hline
\end{tabular}




\section{5-methoxy-4',4'-dimethyl-2-phenyl-1'-tosylspiro[indene-1,3'-pyrrolidine]-}

\section{$3,5^{\prime}(2 H)$-dione (3ab)}

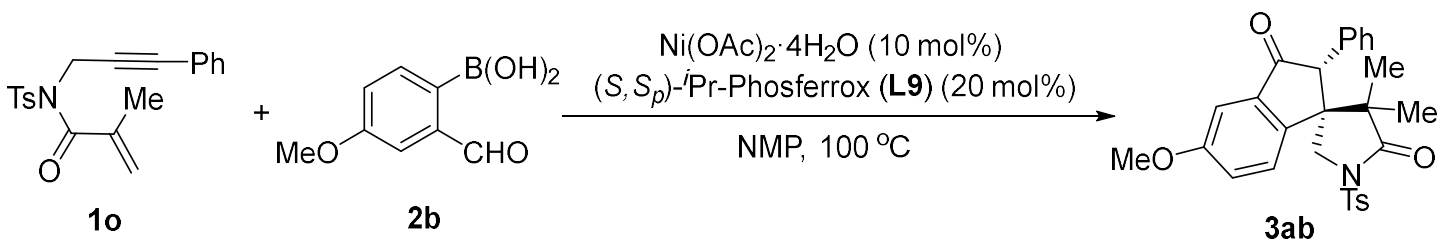

3ab was prepared according to general procedure using $10(0.1 \mathrm{mmol}, 35.3 \mathrm{mg})$ and 2b $(0.3 \mathrm{mmol}, 54.0 \mathrm{mg}$ ) and was purified by silica gel column chromatography (petroleum ether/ethyl acetate $=10 / 1 \sim 5 / 1)$ to obtain $3 \mathrm{ab}(41.1 \mathrm{mg}, 84 \%$ yield).

${ }^{1} \mathrm{H}$ NMR $\left(600 \mathrm{MHz}, \mathrm{CDCl}_{3}\right) \delta 7.89(\mathrm{~d}, J=8.3 \mathrm{~Hz}, 2 \mathrm{H}), 7.38-7.34(\mathrm{~m}, 2 \mathrm{H}), 7.34-7.30(\mathrm{~m}$, $3 \mathrm{H}), 7.23(\mathrm{~d}, J=2.6 \mathrm{~Hz}, 1 \mathrm{H}), 7.00(\mathrm{dd}, J=8.6,2.6 \mathrm{~Hz}, 1 \mathrm{H}), 6.97-6.92(\mathrm{~m}, 2 \mathrm{H}), 6.90(\mathrm{~d}$, $J=8.5 \mathrm{~Hz}, 1 \mathrm{H}), 3.86(\mathrm{~s}, 3 \mathrm{H}), 3.85(\mathrm{~d}, J=2.8 \mathrm{~Hz}, 1 \mathrm{H}), 3.76(\mathrm{~d}, J=10.8 \mathrm{~Hz}, 1 \mathrm{H}), 3.50$ (d, $J=10.8 \mathrm{~Hz}, 1 \mathrm{H}), 2.48(\mathrm{~s}, 3 \mathrm{H}), 1.22(\mathrm{~s}, 3 \mathrm{H}), 0.69$ (s, 3H);

${ }^{13} \mathrm{C}$ NMR $\left(151 \mathrm{MHz}, \mathrm{CDCl}_{3}\right) \delta 203.9,176.7,160.5,149.2,145.5,137.4,137.0,134.7$, 129.8, 129.3, 128.9, 128.2, 128.1, 125.2, 124.8, 105.3, 58.0, 55.7, 53.1, 52.8, 50.0, 22.2, 21.7, 18.6;

HRMS: (ESI) calcd for $\mathrm{C}_{28} \mathrm{H}_{28} \mathrm{NO}_{5} \mathrm{~S}^{+}[\mathrm{M}+\mathrm{H}]^{+}$490.1683; found 490.1677.

The enantiomeric purity was established by HPLC analysis using a chiral column: OD$\mathrm{H}$ column, $30^{\circ} \mathrm{C}, n$-Hexane/ $\mathrm{i}$-Propanol $=70 / 30$ as eluent, $254 \mathrm{~nm}, 1 \mathrm{~mL} / \mathrm{min}$. $\mathrm{tR}=9 \mathrm{~min}$ (major), 18 min (minor).

Optical Rotation: $[\alpha]_{D}^{28}-7.5$ (c 5.0, MeCN) for $90 \%$ ee.

Absolute stereochemistry was determined through analogy with $\mathbf{3 a}$. 
$\mathrm{mAU}$

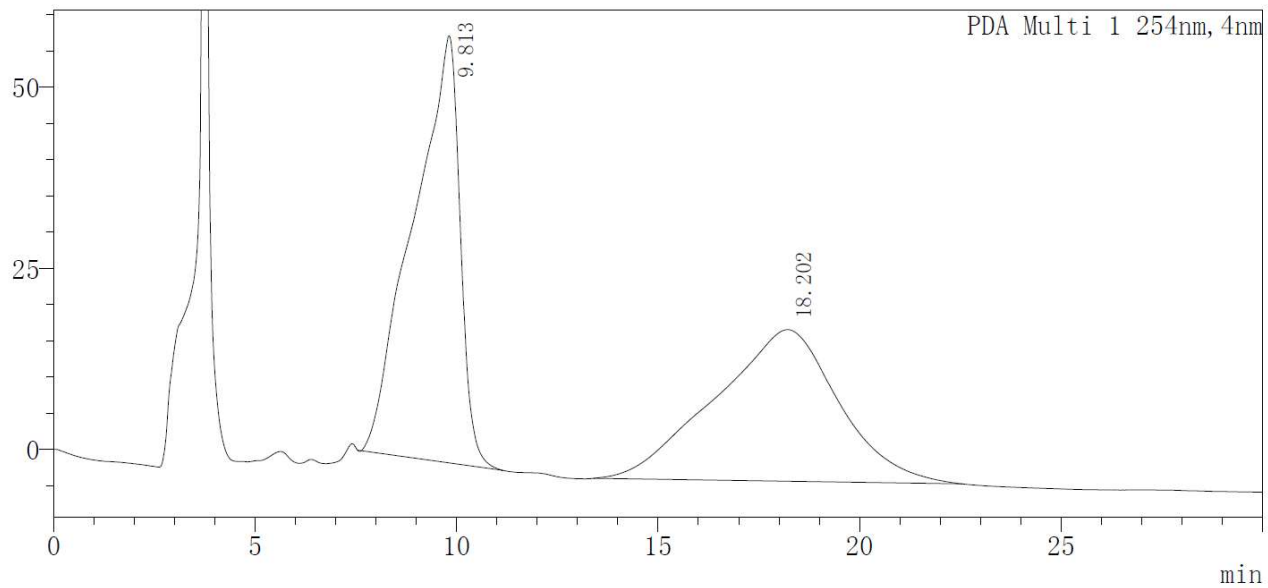

〈峰表〉

\begin{tabular}{|c|c|c|c|c|c|c|c|}
\hline \begin{tabular}{|l|} 
峰号 \\
\end{tabular} & 保留时间 & 面积 & 高度 & 浓度 & 浓度单位 & 标记 & 化合物名 \\
\hline$\uparrow 1$ & \9.813 & \4 4748135 & 个 58918 & 0.000 & & M & \\
\hline 2 & 18.202 & 4443292 & 20898 & 0.000 & & M & \\
\hline 总计 & & 9191427 & 79816 & & & & \\
\hline m & & & ht & & & & \\
\hline
\end{tabular}

retention time

mAU

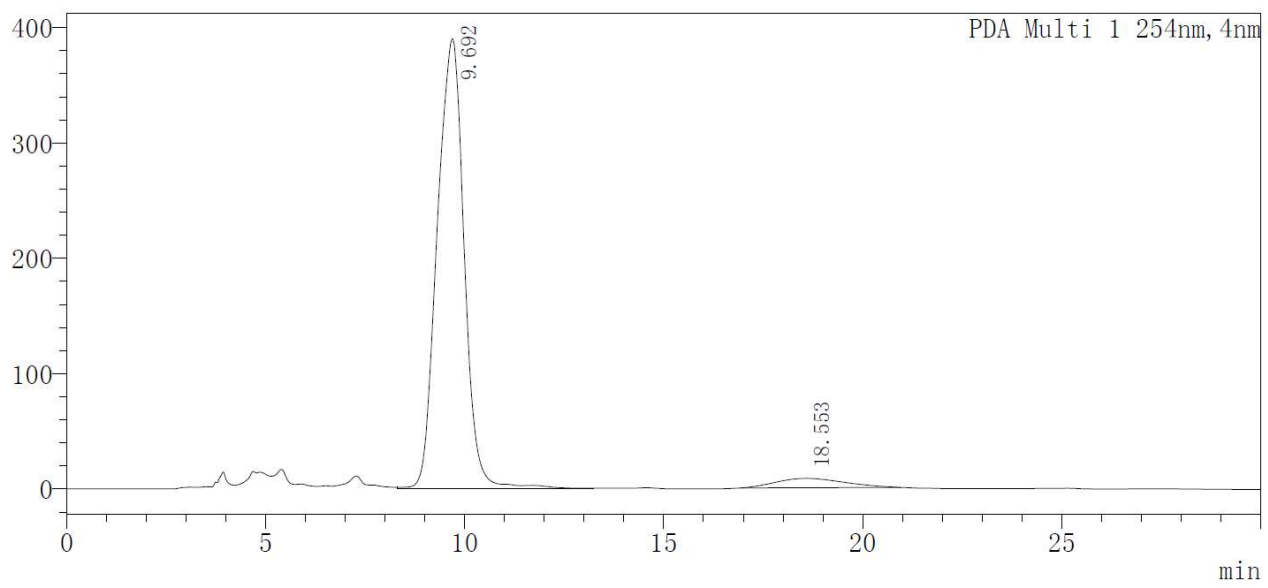

〈峰表〉

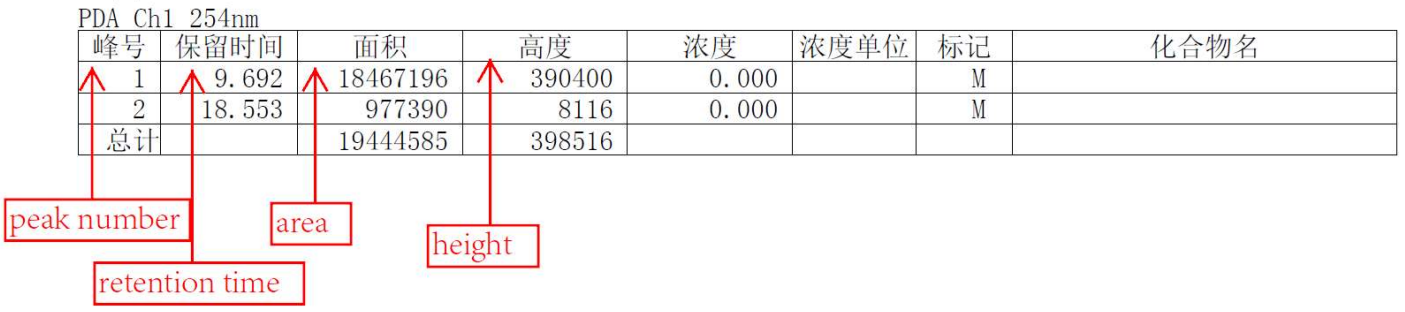




\section{5-(benzyloxy)-4',4'-dimethyl-2-phenyl-1'-tosylspiro[indene-1,3'-pyrrolidine]-}

\section{$3,5^{\prime}(2 H)$-dione (3ac)}

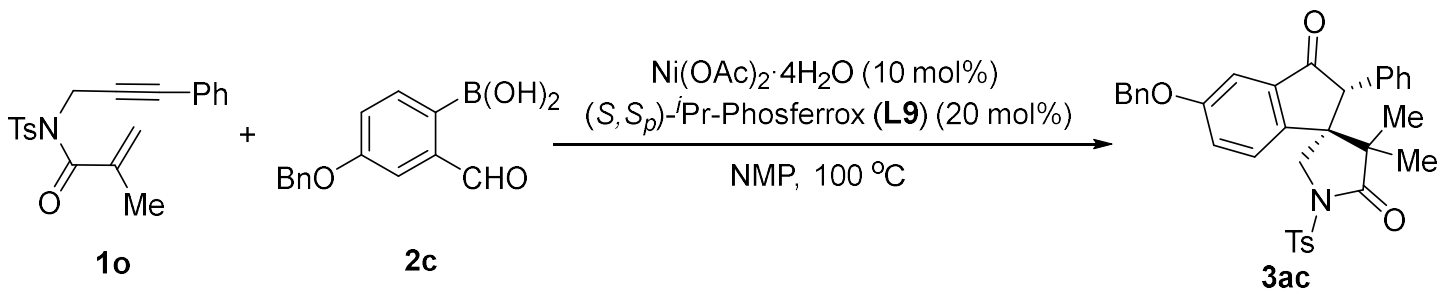

3ac was prepared according to general procedure using $10(0.1 \mathrm{mmol}, 35.3 \mathrm{mg})$ and 2c $(0.3 \mathrm{mmol}, 76.8 \mathrm{mg})$ and was purified by silica gel column chromatography (petroleum ether/ethyl acetate $=10 / 1 \sim 5 / 1)$ to obtain 3ac $(50.3 \mathrm{mg}, 89 \%$ yield).

${ }^{1} \mathrm{H}$ NMR $\left(600 \mathrm{MHz}, \mathrm{CDCl}_{3}\right)$ ठ 7.92-7.87 (m, 2H), 7.45-7.40 (m, 4H), 7.40-7.30 (m, 7H), $7.07(\mathrm{dd}, J=8.6,2.6 \mathrm{~Hz}, 1 \mathrm{H}), 6.96-6.93(\mathrm{~m}, 2 \mathrm{H}), 6.90(\mathrm{~d}, J=8.6 \mathrm{~Hz}, 1 \mathrm{H}), 5.09(\mathrm{~s}, 2 \mathrm{H})$, 3.85 (s, 1H), 3.77 (d, J = $10.8 \mathrm{~Hz}, 1 \mathrm{H}), 3.50$ (d, J = $10.9 \mathrm{~Hz}, 1 \mathrm{H}), 2.47$ (s, 3H), 1.23 (s, $3 \mathrm{H}), 0.70$ (s, 3H);

${ }^{13} \mathrm{C}$ NMR $\left(151 \mathrm{MHz}, \mathrm{CDCl}_{3}\right) \delta 203.8,176.7,159.7,149.4,145.5,137.4,137.0,135.8$, $134.7,129.7,129.3,128.9,128.7,128.4,128.2,128.1,127.7,125.7,124.9,106.4$, $70.5,58.0,53.2,52.8,50.0,22.2,21.8,18.7$

HRMS: (ESI) calcd for $\mathrm{C}_{34} \mathrm{H}_{32} \mathrm{NO}_{5} \mathrm{~S}^{+}[\mathrm{M}+\mathrm{H}]^{+}$566.1996; found 566.1998.

The enantiomeric purity was established by HPLC analysis using a chiral column: OD$\mathrm{H}$ column, $30^{\circ} \mathrm{C}, n$-Hexane/i-Propanol $=70 / 30$ as eluent, $254 \mathrm{~nm}, 1 \mathrm{~mL} / \mathrm{min} . \mathrm{tR}=20$ $\min$ (major), 25 min (minor).

Optical Rotation: $[\alpha]_{D}^{28} 38.3$ (c 5.0, MeCN) for 93\% ee. Absolute stereochemistry was determined through analogy with $\mathbf{3 a}$. 
mAU

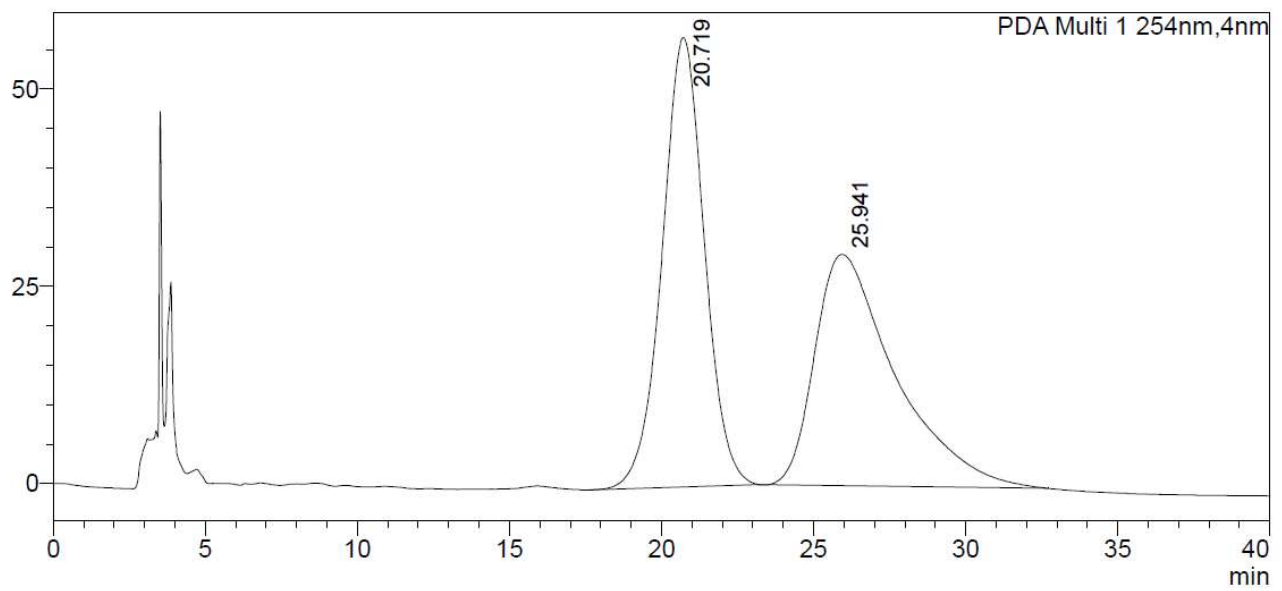

<Peak Table>

PDA Ch1 254nm

\begin{tabular}{|r|r|r|r|r|r|r|r|}
\hline Peak\# & Ret. Time & \multicolumn{1}{c|}{ Area } & Height & Conc. & Unit & Mark & Name \\
\hline 1 & 20.719 & 5503799 & 56939 & 0.000 & & $\mathrm{M}$ & \\
\hline 2 & 25.941 & 5386884 & 29316 & 0.000 & & $\mathrm{M}$ & \\
\hline Total & & 10890683 & 86255 & & & &
\end{tabular}

mAU

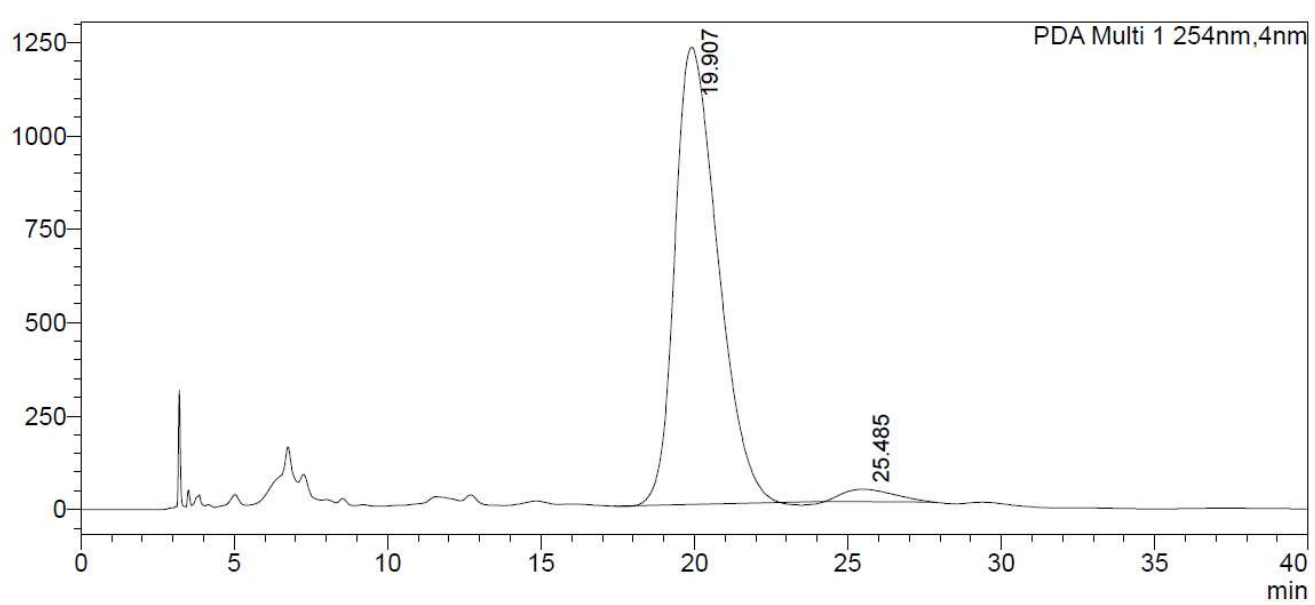

<Peak Table>

PDA Ch1 254nm

Peak\# Ret. Time

\begin{tabular}{|r|r|r|r|r|r|r|r|}
\hline Peak\# & Ret. Time & \multicolumn{1}{|c|}{ Area } & Height & Conc. & Unit & Mark & Name \\
\hline 1 & 19.907 & 118994934 & 1223607 & 0.000 & & $\mathrm{M}$ & \\
\hline 2 & 25.485 & 3867717 & 33101 & 0.000 & & $\mathrm{M}$ & \\
\hline Total & & 122862651 & 1256709 & & & &
\end{tabular}




\section{5-chloro-4',4'-dimethyl-2-phenyl-1'-tosylspiro[indene-1,3'-pyrrolidine]-3,5'(2H)-}

dione (3ad)

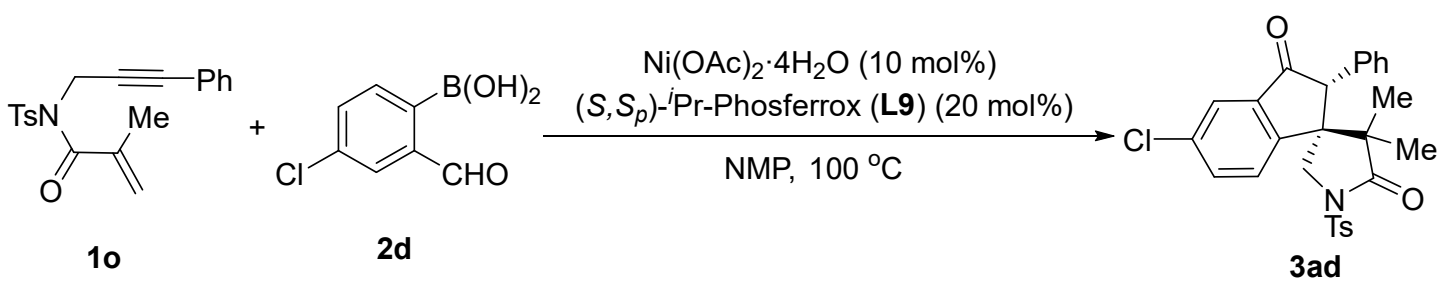

3ad was prepared according to general procedure using $10(0.1 \mathrm{mmol}, 35.3 \mathrm{mg})$ and 2d $(0.3 \mathrm{mmol}, 55.2 \mathrm{mg})$ and was purified by silica gel column chromatography (petroleum ether/ethyl acetate $=10 / 1 \sim 5 / 1)$ to obtain $3 \mathrm{ad}(30.1 \mathrm{mg}, 61 \%$ yield $)$.

${ }^{1} \mathrm{H}$ NMR $\left(600 \mathrm{MHz}, \mathrm{CDCl}_{3}\right) \delta 7.89(\mathrm{~d}, J=8.3 \mathrm{~Hz}, 2 \mathrm{H}), 7.79(\mathrm{~d}, J=2.1 \mathrm{~Hz}, 1 \mathrm{H}), 7.40$ (dd, $J=8.4,2.1 \mathrm{~Hz}, 1 \mathrm{H}), 7.37(\mathrm{~d}, J=8.1 \mathrm{~Hz}, 2 \mathrm{H}), 7.35-7.32(\mathrm{~m}, 3 \mathrm{H}), 7.01(\mathrm{~d}, J=8.3$ $\mathrm{Hz}, 1 \mathrm{H}), 6.96-6.89(\mathrm{~m}, 2 \mathrm{H}), 3.87(\mathrm{~s}, 1 \mathrm{H}), 3.77(\mathrm{~d}, J=10.9 \mathrm{~Hz}, 1 \mathrm{H}), 3.50(\mathrm{~d}, J=10.9$ $\mathrm{Hz}, 1 \mathrm{H}), 2.48(\mathrm{~s}, 3 \mathrm{H}), 1.22(\mathrm{~s}, 3 \mathrm{H}), 0.70(\mathrm{~s}, 3 \mathrm{H})$; ${ }^{13} \mathrm{C}$ NMR $\left(151 \mathrm{MHz}, \mathrm{CDCl}_{3}\right) \delta 202.6,176.3,154.5,145.7,137.4,136.4,136.0,135.9$, 134.6, 129.8, 129.4, 128.9, 128.3, 128.2, 125.3, 124.2, 57.7, 53.4, 52.4, 50.0, 22.1, 21.7, 18.6;

HRMS: (ESI) calcd for $\mathrm{C}_{27} \mathrm{H}_{25} \mathrm{CINO}_{4} \mathrm{~S}^{+}[\mathrm{M}+\mathrm{H}]^{+}$494.1187; found 494.1182.

The enantiomeric purity was established by HPLC analysis using a chiral column: AD$\mathrm{H}$ column, $30^{\circ} \mathrm{C}, n$-Hexane/i-Propanol $=80 / 20$ as eluent, $254 \mathrm{~nm}, 1 \mathrm{~mL} / \mathrm{min} . \mathrm{tR}=16$ $\min$ (major), 18 min (minor).

Optical Rotation: $[\alpha]_{D}^{28}-3.8$ (c 5.0, MeCN) for $90 \%$ ee.

Absolute stereochemistry was determined through analogy with $\mathbf{3 a}$. 
mAU

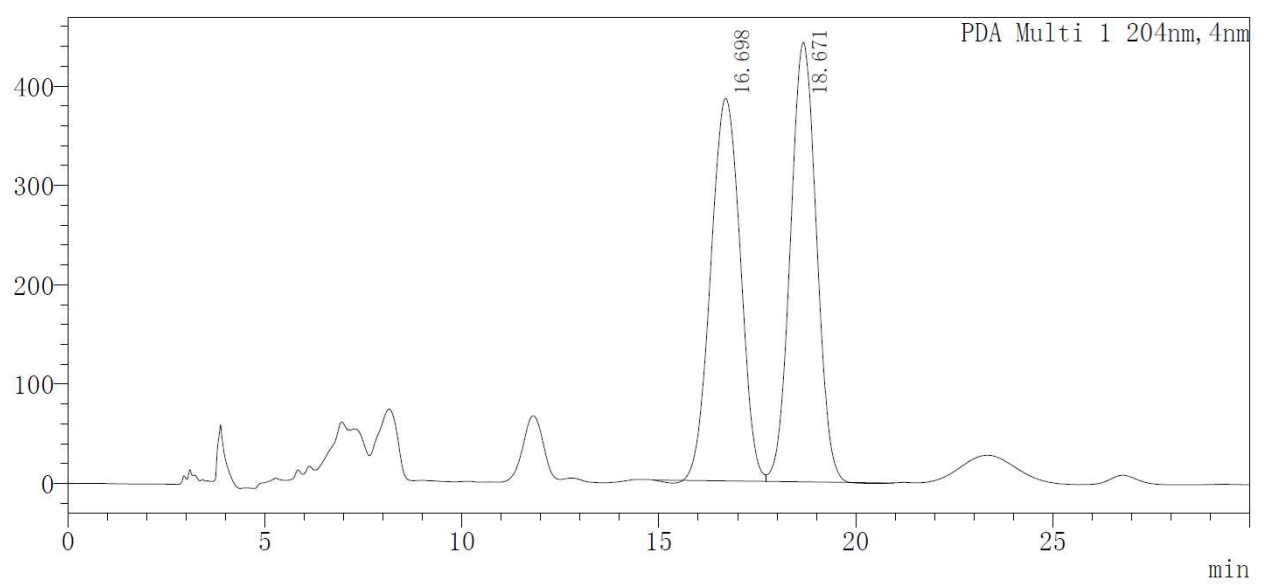

〈峰表〉

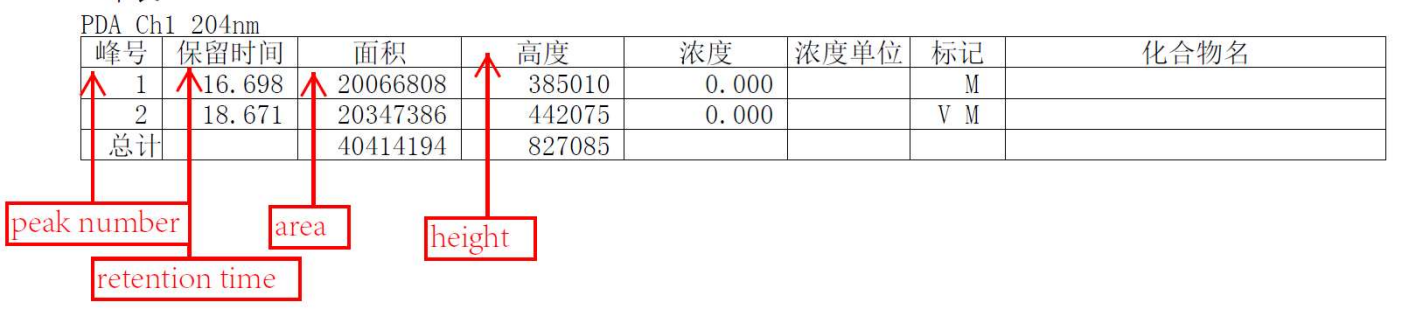

mAU

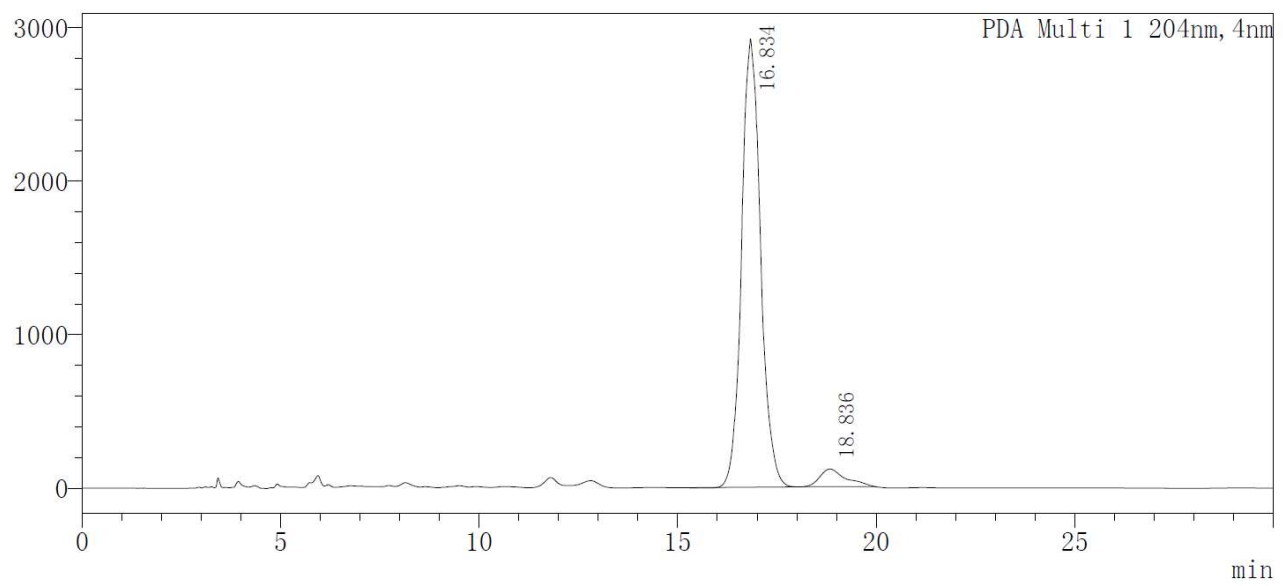

〈峰表〉

\begin{tabular}{|c|c|c|c|c|c|c|c|}
\hline 峰号 & 保留时间 & 面积 & 高度 & 浓度 & 浓度单位 & 标记 & 化合物名 \\
\hline \$ 1 & 凡16.8341 & 196766605 & 2920532 & 94.872 & & M & \\
\hline 2 & 18.836 & 5230589 & 114641 & 5.128 & & M & \\
\hline 总计 & & 101997194 & 3035173 & & & & \\
\hline
\end{tabular}

\begin{tabular}{|l|l|}
\hline peak number & area \\
\hline
\end{tabular}

retention time 


\section{5-hydroxy-4',4'-dimethyl-2-phenyl-1'-tosylspiro[indene-1,3'-pyrrolidine]-3,5'(2H)-}

dione (3ae)

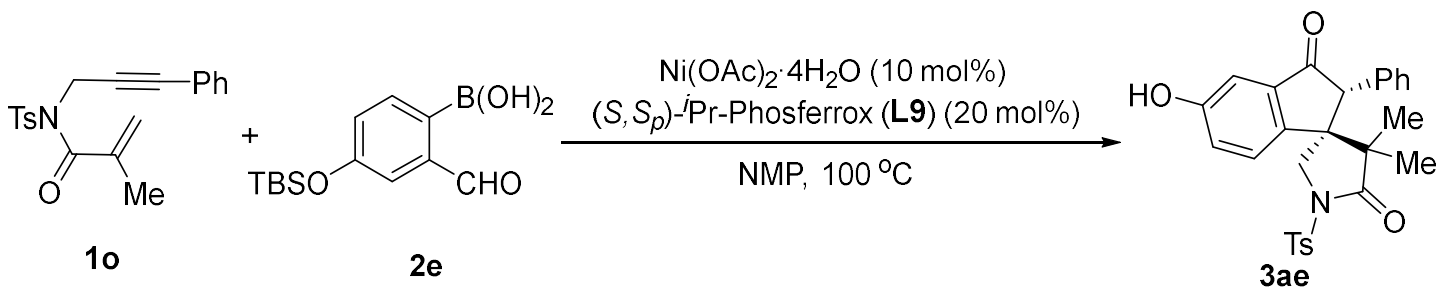

3ae was prepared according to general procedure using $10(0.1 \mathrm{mmol}, 35.3 \mathrm{mg})$ and 2e $(0.3 \mathrm{mmol}, 84.0 \mathrm{mg})$ and was purified by silica gel column chromatography (petroleum ether/ethyl acetate $=10 / 1 \sim 5 / 1)$ to obtain 3 ae $(26.6 \mathrm{mg}, 56 \%$ yield $)$.

${ }^{1} \mathrm{H}$ NMR $\left(600 \mathrm{MHz}, \mathrm{CDCl}_{3}\right) \delta 7.89(\mathrm{~d}, J=8.3 \mathrm{~Hz}, 2 \mathrm{H}), 7.36(\mathrm{~d}, J=8.1 \mathrm{~Hz}, 2 \mathrm{H}), 7.32-$ $7.29(\mathrm{~m}, 3 \mathrm{H}), 7.21-7.18(\mathrm{~m}, 1 \mathrm{H}), 6.95-6.88(\mathrm{~m}, 4 \mathrm{H}), 3.84(\mathrm{~s}, 1 \mathrm{H}), 3.78(\mathrm{~d}, J=10.8 \mathrm{~Hz}$, 1H), 3.48 (d, J = $10.8 \mathrm{~Hz}, 1 \mathrm{H}), 2.46(\mathrm{~s}, 3 \mathrm{H}), 1.83(\mathrm{~s}, 1 \mathrm{H}), 1.20(\mathrm{~s}, 3 \mathrm{H}), 0.68(\mathrm{~s}, 3 \mathrm{H})$; ${ }^{13} \mathrm{C}$ NMR $\left(151 \mathrm{MHz}, \mathrm{CDCl}_{3}\right) \delta 204.3,177.0,157.2,148.7,145.7,137.4,136.8,134.6$, 129.8, 129.3, 128.9, 128.1, 128.1, 124.9, 124.7, 109.2, 58.0, 53.1, 52.9, 50.2, 22.1, 21.7, 18.6;

HRMS: (ESI) calcd for $\mathrm{C}_{27} \mathrm{H}_{26} \mathrm{NO}_{5} \mathrm{~S}^{+}[\mathrm{M}+\mathrm{H}]^{+}$476.1526; found 476.1527.

The enantiomeric purity was established by HPLC analysis using a chiral column: OD$\mathrm{H}$ column, $30^{\circ} \mathrm{C}, n$-Hexane/i-Propanol $=80 / 20$ as eluent, $254 \mathrm{~nm}, 1 \mathrm{~mL} / \mathrm{min}$. $\mathrm{tR}=7 \mathrm{~min}$ (major), 19 min (minor).

Optical Rotation: $[\alpha]_{D}{ }^{28} 15.5$ (c 5.0, MeCN) for 93\% ee.

Absolute stereochemistry was determined through analogy with $\mathbf{3 a}$. 
$\mathrm{mAU}$

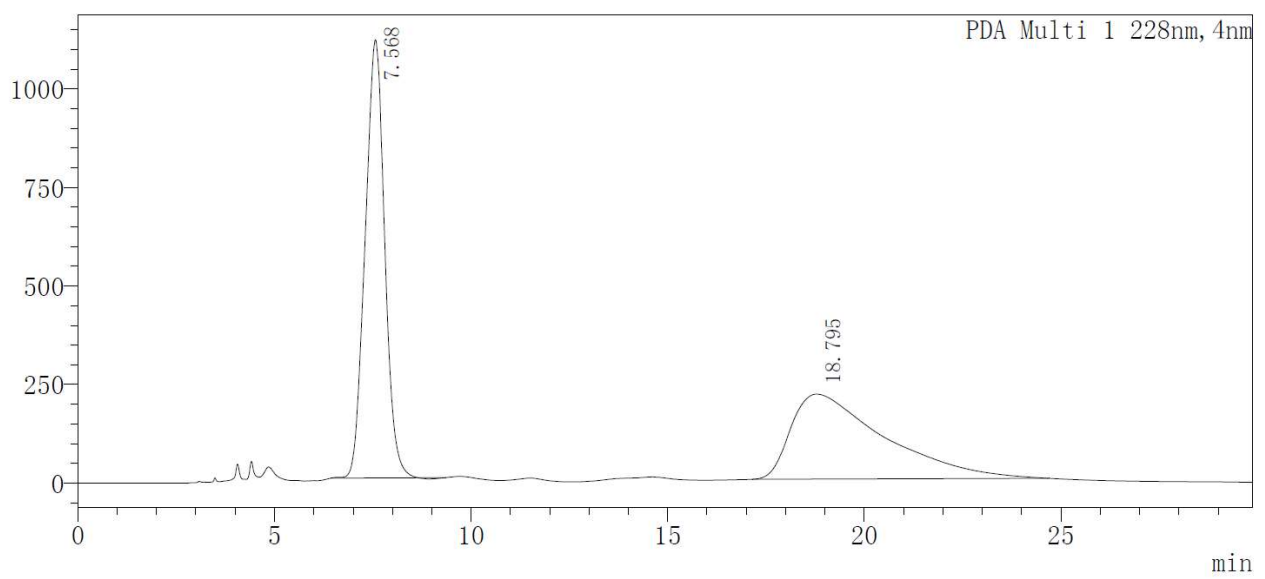

〈峰表〉

PDA Ch1 228nm

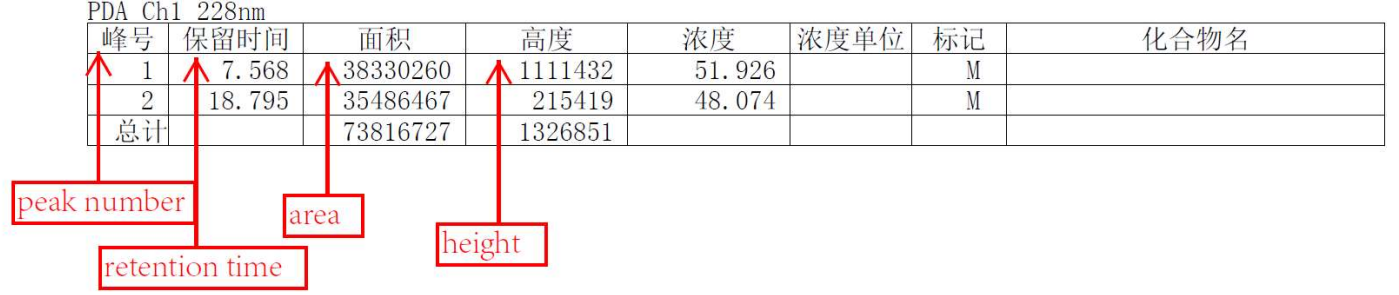

$\mathrm{mAU}$

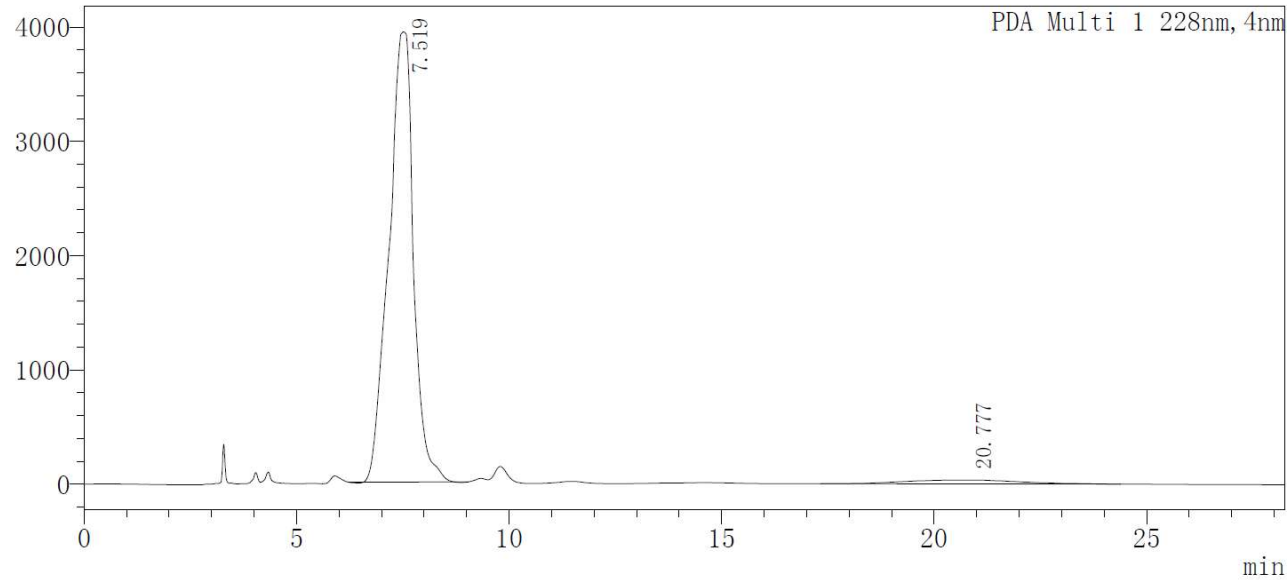

〈峰表〉

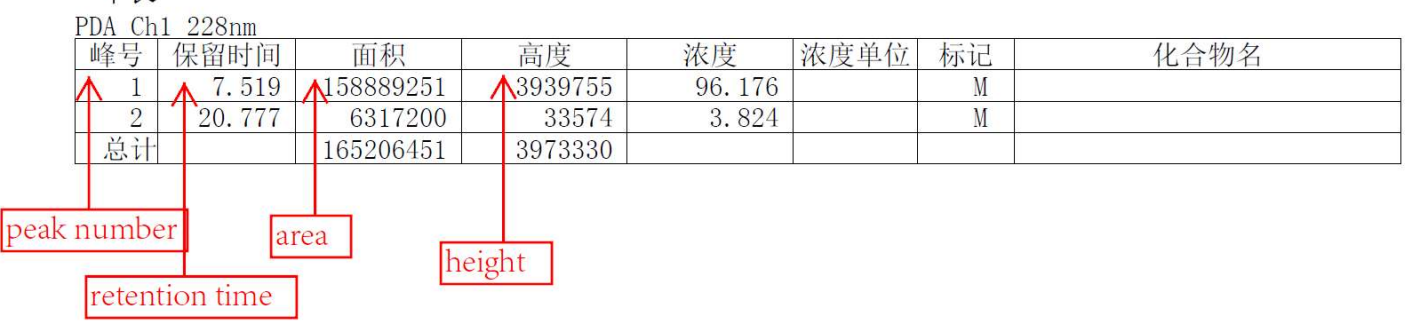




\section{6-fluoro-4',4'-dimethyl-2-phenyl-1'-tosylspiro[indene-1,3'-pyrrolidine]-3,5'(2H)-}

dione (3af)

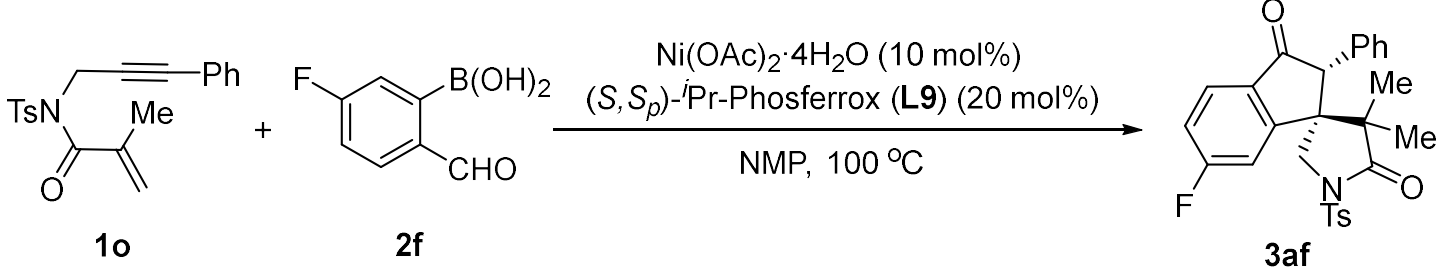

3af was prepared according to general procedure using $10(0.1 \mathrm{mmol}, 35.3 \mathrm{mg})$ and $2 \mathrm{f}(0.3 \mathrm{mmol}, 50.1 \mathrm{mg})$ and was purified by silica gel column chromatography (petroleum ether/ethyl acetate $=10 / 1 \sim 5 / 1)$ to obtain 3 af $(36.7 \mathrm{mg}, 77 \%$ yield $)$. ${ }^{1} \mathrm{H}$ NMR $\left(600 \mathrm{MHz}, \mathrm{CDCl}_{3}\right)$ ठ 7.96-7.90 (m, 2H), $7.84(\mathrm{dd}, J=8.5,5.3 \mathrm{~Hz}, 1 \mathrm{H}), 7.41-$ $7.37(\mathrm{~m}, 2 \mathrm{H}), 7.35-7.31(\mathrm{~m}, 3 \mathrm{H}), 7.15(\mathrm{td}, J=8.4,2.2 \mathrm{~Hz}, 1 \mathrm{H}), 6.97-6.90(\mathrm{~m}, 2 \mathrm{H}), 6.37$ (dd, $J=8.8,2.1 \mathrm{~Hz}, 1 \mathrm{H}), 3.87(\mathrm{~s}, 1 \mathrm{H}), 3.71(\mathrm{~d}, J=11.1 \mathrm{~Hz}, 1 \mathrm{H}), 3.55(\mathrm{~d}, J=11.1 \mathrm{~Hz}$, $1 \mathrm{H}), 2.49(\mathrm{~s}, 3 \mathrm{H}), 1.30(\mathrm{~s}, 3 \mathrm{H}), 0.70(\mathrm{~s}, 3 \mathrm{H})$;

${ }^{13} \mathrm{C}$ NMR $\left(151 \mathrm{MHz}, \mathrm{CDCl}_{3}\right) \delta$ 202.05, 176.13, 168.48, 166.76, 159.35 (d, J = $\left.9.1 \mathrm{~Hz}\right)$, 146.04, 136.50, 134.48, 132.31 (d, J = 2.1 Hz), 129.95, 129.40, 128.89, 128.25, 128.02 $126.76(\mathrm{~d}, J=10.6 \mathrm{~Hz}), 117.67(\mathrm{~d}, J=23.5 \mathrm{~Hz}), 110.92(\mathrm{~d}, J=23.5 \mathrm{~Hz}), 57.52,53.49$, $52.50,49.88,22.38,21.71,18.44$

${ }^{19} \mathrm{~F} \mathrm{NMR}\left(376 \mathrm{MHz}, \mathrm{CDCl}_{3}\right) \delta-98.3(\mathrm{~m})$

HRMS: (ESI) calcd for $\mathrm{C}_{27} \mathrm{H}_{25} \mathrm{FNO}_{4} \mathrm{~S}^{+}[\mathrm{M}+\mathrm{H}]^{+}$478.1483; found 478.1480 .

The enantiomeric purity was established by HPLC analysis using a chiral column: AD$\mathrm{H}$ column, $30^{\circ} \mathrm{C}, n$-Hexane/i-Propanol $=80 / 20$ as eluent, $254 \mathrm{~nm}, 1 \mathrm{~mL} / \mathrm{min} . \mathrm{tR}=13$ min (major), 29 min (minor).

Optical Rotation: [a] $]_{D}^{28} 12.2$ (c 5.0, MeCN) for 95\% ee. Absolute stereochemistry was determined through analogy with $\mathbf{3 a}$. 
mAU

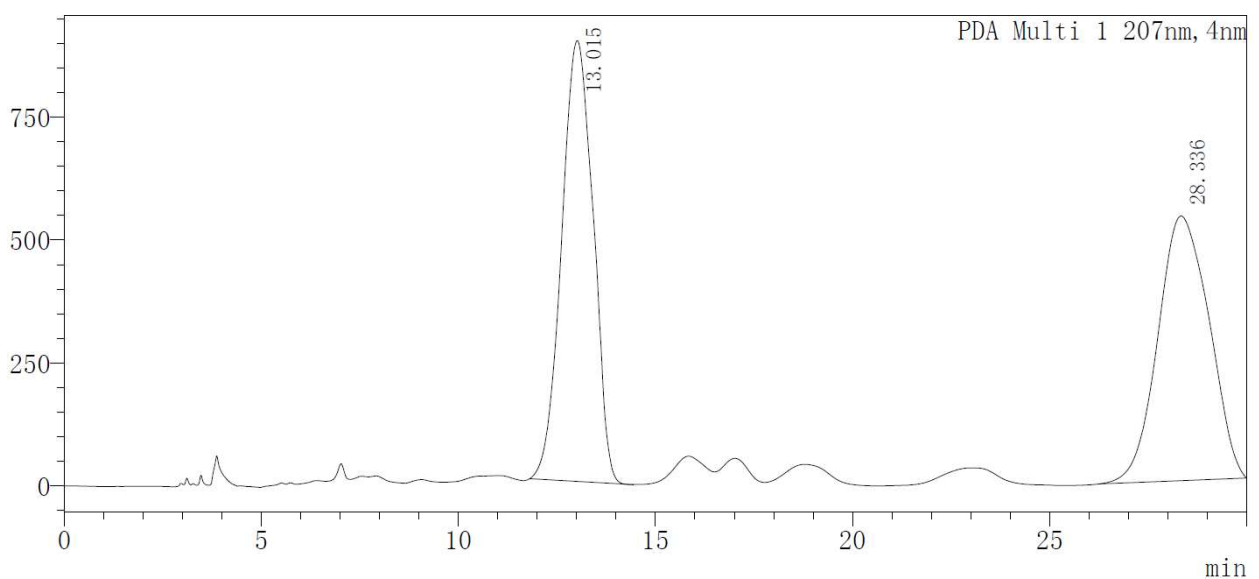

〈峰表〉
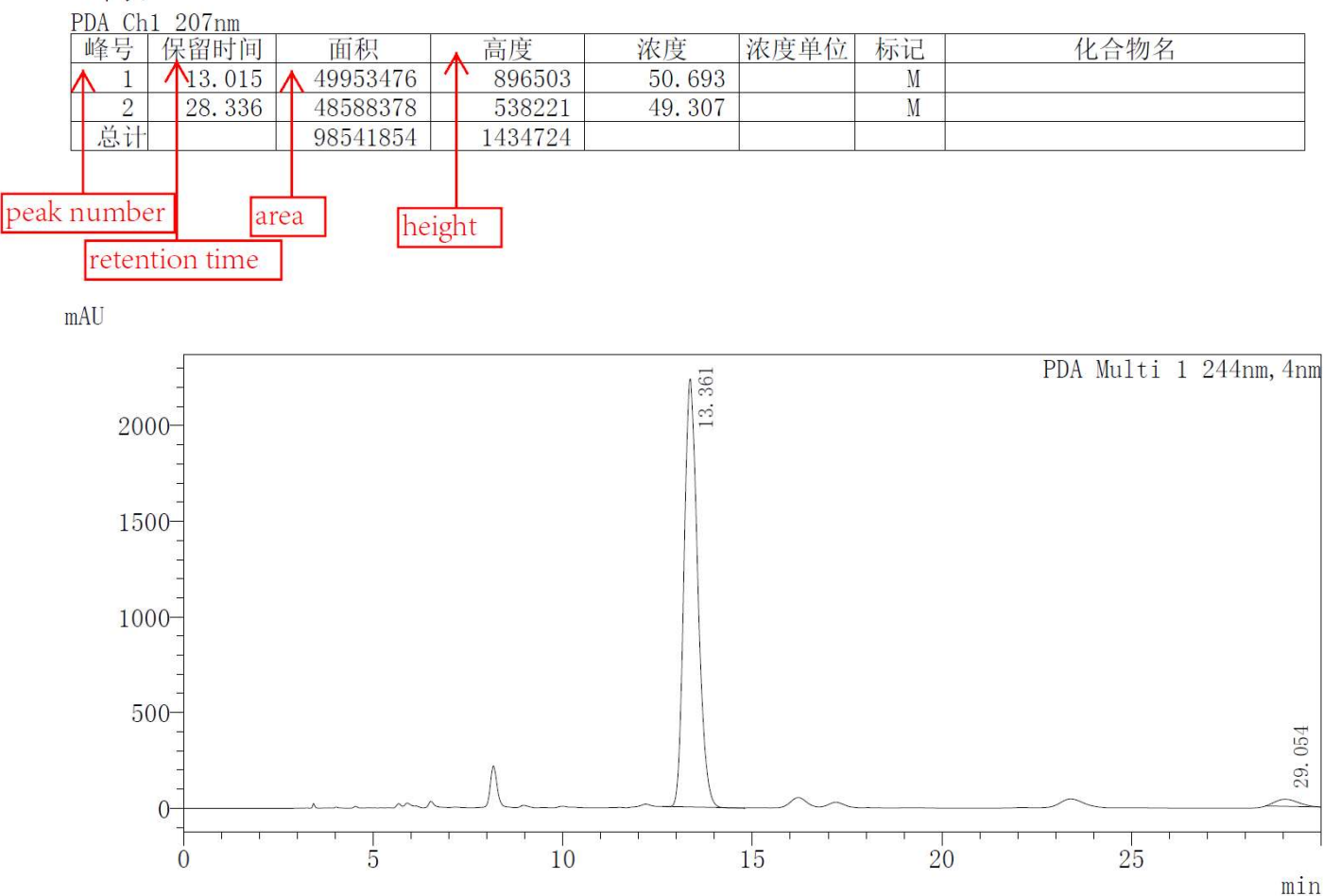

〈峰表〉

PDA Ch1 244nm 峰号

面积

\begin{tabular}{|c|}
\hline 高度 \\
\hline $\begin{array}{r}2238239 \\
27021\end{array}$ \\
\hline
\end{tabular}

\begin{tabular}{|c|c|c|}
\hline 度 & 浓度单位 & 标记 \\
\hline 0.000 & & $\mathrm{M}$ \\
\hline 0.000 & & $\mathrm{M}$
\end{tabular}
2275470 0.000

\begin{tabular}{|r|r|r|r|}
\hline 2 & 29.054 & 1584301 & 37231 \\
\hline 总计 & & 57747973 & 2275470
\end{tabular}

peak number

area

height

retention time 


\section{5,6-dimethoxy-4',4'-dimethyl-2-phenyl-1'-tosylspiro[indene-1,3'-pyrrolidine]-}

\section{$3,5^{\prime}(2 H)$-dione (3ag)}

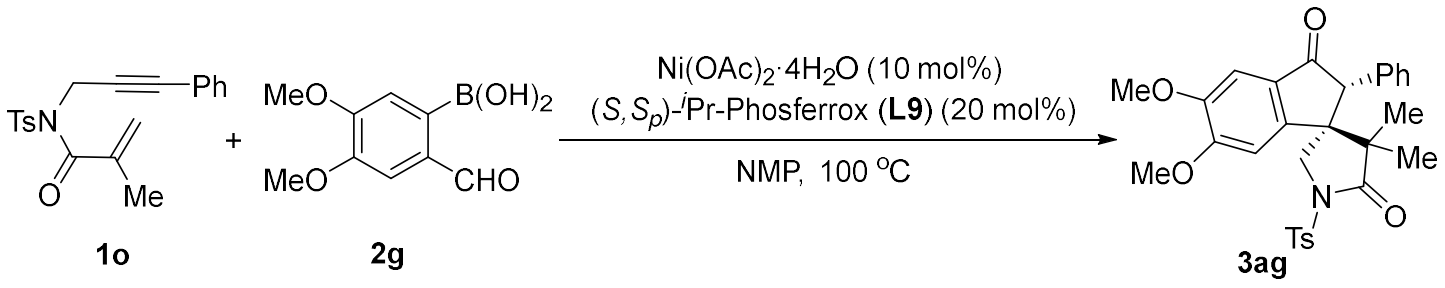

3ag was prepared according to general procedure using $10(0.1 \mathrm{mmol}, 35.3 \mathrm{mg})$ and 2g ( $0.3 \mathrm{mmol}, 63.0 \mathrm{mg})$ and was purified by silica gel column chromatography (petroleum ether/ethyl acetate $=10 / 1 \sim 5 / 1)$ to obtain $3 \mathrm{ag}(41.1 \mathrm{mg}, 79 \%$ yield $)$.

${ }^{1} \mathrm{H}$ NMR $\left(600 \mathrm{MHz}, \mathrm{CDCl}_{3}\right)$ ठ 7.93-7.88 (m, 2H), 7.36-7.31 (m, 5H), 7.24 (s, 1H), 6.99$6.96(\mathrm{~m}, 2 \mathrm{H}), 6.89(\mathrm{~s}, 1 \mathrm{H}), 3.94(\mathrm{~s}, 3 \mathrm{H}), 3.88(\mathrm{~d}, J=10.7 \mathrm{~Hz}, 1 \mathrm{H}), 3.82(\mathrm{~s}, 1 \mathrm{H}), 3.77(\mathrm{~s}$, 3H), $3.44(\mathrm{~d}, J=10.8 \mathrm{~Hz}, 1 \mathrm{H}), 2.44(\mathrm{~s}, 3 \mathrm{H}), 1.22(\mathrm{~s}, 3 \mathrm{H}), 0.73(\mathrm{~s}, 3 \mathrm{H})$;

${ }^{13} \mathrm{C} \mathrm{NMR}\left(151 \mathrm{MHz}, \mathrm{CDCl}_{3}\right) \delta 202.5,177.0,156.7,152.0,150.7,145.5,137.2,135.0$, $129.7,129.3,129.0,128.9,128.2,128.0,104.8,104.2,57.2,56.4,56.2,53.3,52.5$, $50.0,22.2,21.7,18.4$

HRMS: (ESI) calcd for $\mathrm{C}_{29} \mathrm{H}_{30} \mathrm{NO}_{6} \mathrm{~S}^{+}[\mathrm{M}+\mathrm{H}]^{+} 520.1788$; found 520.1783 .

The enantiomeric purity was established by HPLC analysis using a chiral column: OD$\mathrm{H}$ column, $30^{\circ} \mathrm{C}, n$-Hexane/i-Propanol $=60 / 40$ as eluent, $254 \mathrm{~nm}, 1 \mathrm{~mL} / \mathrm{min}$. $\mathrm{tR}=7 \mathrm{~min}$ (major), 12 min (minor).

Optical Rotation: $[\alpha]_{D}^{28}-39.7$ (c 5.0, MeCN) for $98 \%$ ee.

Absolute stereochemistry was determined through analogy with $\mathbf{3 a}$. 
$\mathrm{mAU}$

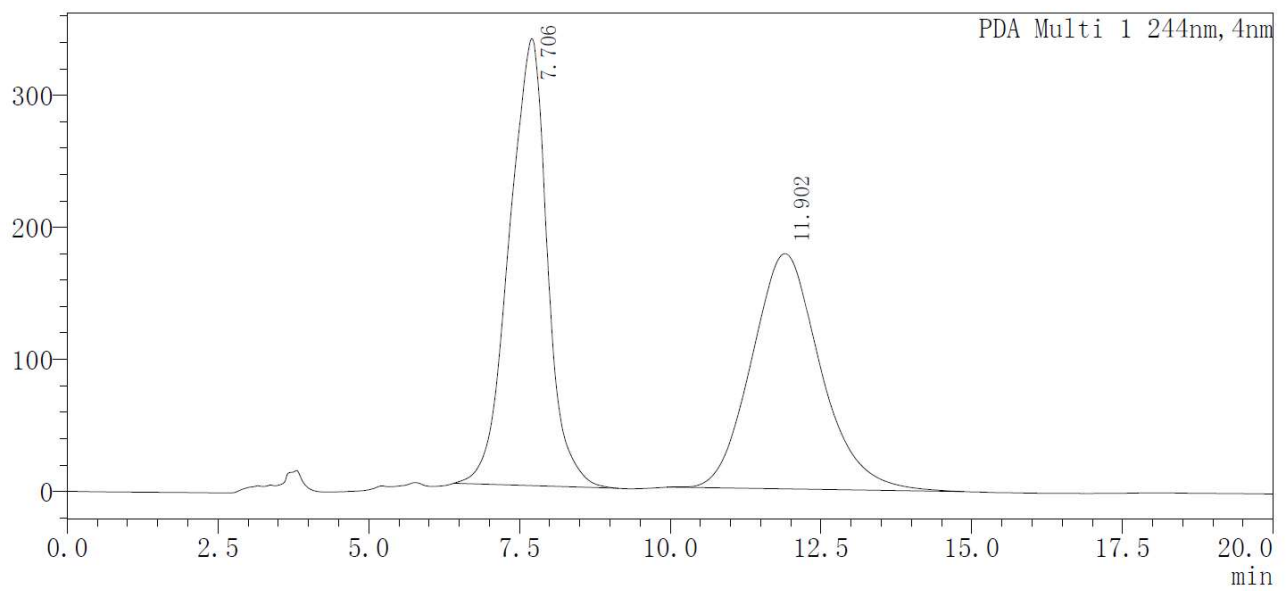

〈峰表〉

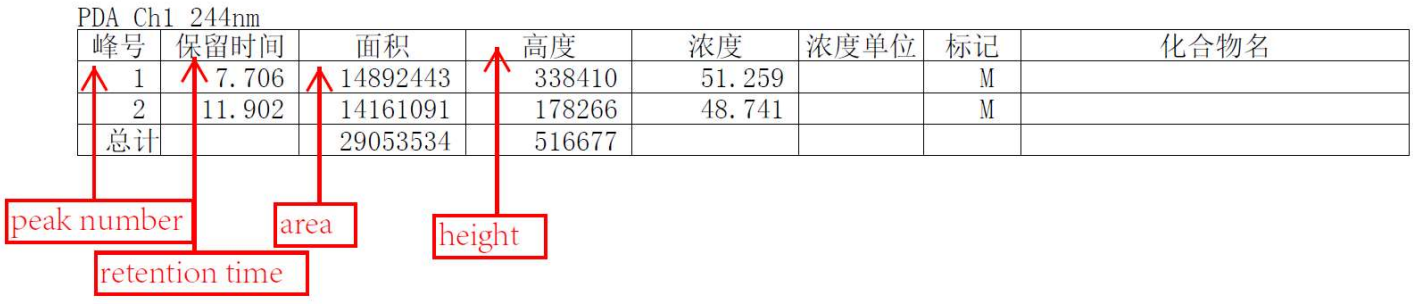

mAU

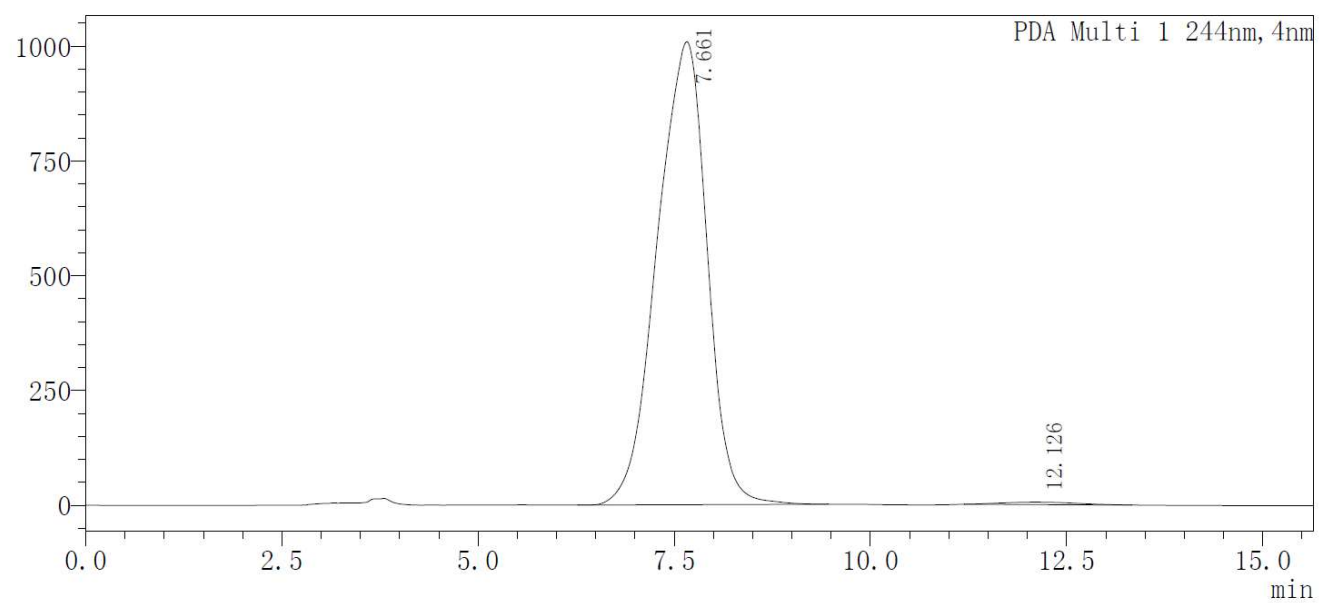

〈峰表〉

PDA Ch1 244nm

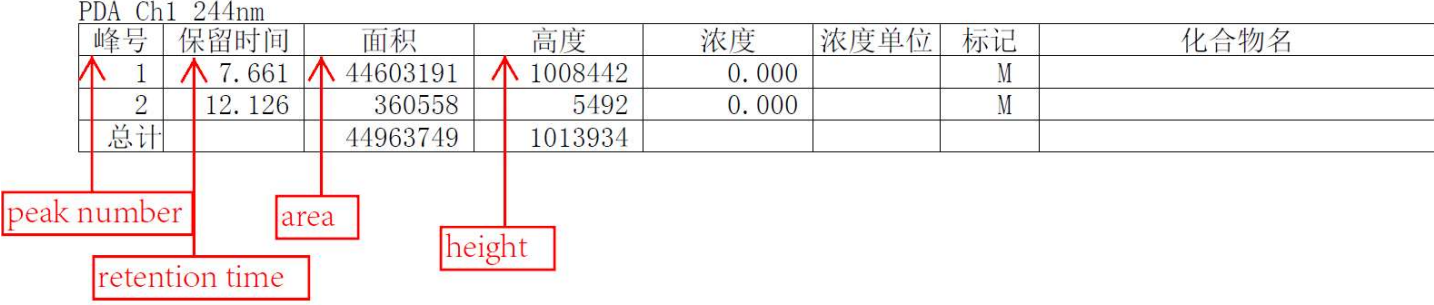




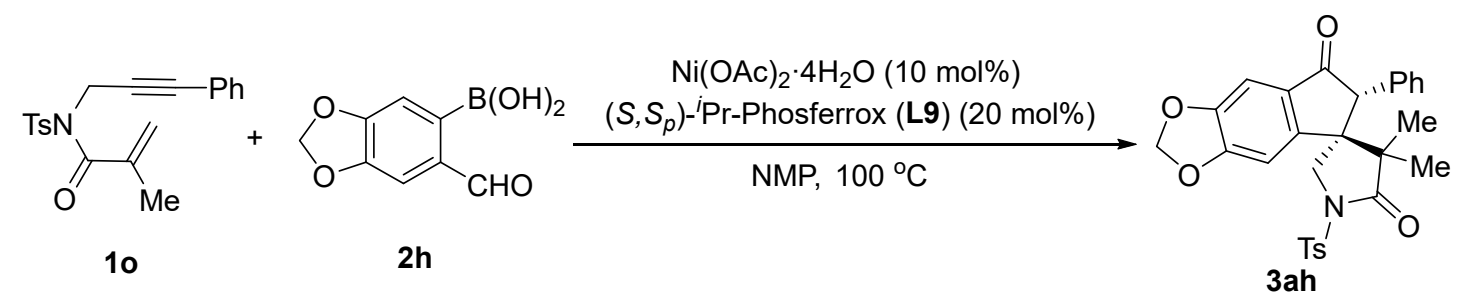

3ah was prepared according to general procedure using $10(0.1 \mathrm{mmol}, 35.3 \mathrm{mg})$ and $2 \mathrm{~h}(0.3 \mathrm{mmol}, 58.0 \mathrm{mg}$ ) and was purified by silica gel column chromatography (petroleum ether/ethyl acetate $=10 / 1 \sim 5 / 1)$ to obtain 3 ah $(36.7 \mathrm{mg}, 73 \%$ yield).

${ }^{1} \mathrm{H}$ NMR $\left(600 \mathrm{MHz}, \mathrm{CDCl}_{3}\right)$ ठ 7.92-7.87 (m, 2H), 7.39-7.34 (m, 2H), 7.33-7.29 (m, 3H), $7.14(\mathrm{~s}, 1 \mathrm{H}), 6.98-6.91(\mathrm{~m}, 2 \mathrm{H}), 6.24(\mathrm{~s}, 1 \mathrm{H}), 6.10(\mathrm{~d}, J=1.1 \mathrm{~Hz}, 1 \mathrm{H}), 6.07(\mathrm{~d}, J=1.1$ $\mathrm{Hz}, 1 \mathrm{H}), 3.82(\mathrm{~s}, 1 \mathrm{H}), 3.71(\mathrm{~d}, J=10.9 \mathrm{~Hz}, 1 \mathrm{H}), 3.47(\mathrm{~d}, J=11.0 \mathrm{~Hz}, 1 \mathrm{H}), 2.46(\mathrm{~s}, 3 \mathrm{H})$, $1.24(\mathrm{~s}, 3 \mathrm{H})$;

${ }^{13} \mathrm{C}$ NMR $\left(151 \mathrm{MHz}, \mathrm{CDCl}_{3}\right) \delta 201.7,176.6,155.4,154.0,149.5,145.7,137.0,134.6$, $131.0,129.9,129.3,128.9,128.1$, 103.3, 102.8, 102.5, 57.7, 53.2, 52.6, 50.0, 22.4, 21.8, 18.4;

HRMS: (ESI) calcd for $\mathrm{C}_{28} \mathrm{H}_{26} \mathrm{NO}_{6} \mathrm{~S}^{+}[\mathrm{M}+\mathrm{H}]^{+}$504.1475; found 504.1476.

The enantiomeric purity was established by HPLC analysis using a chiral column: AD$\mathrm{H}$ column, $30^{\circ} \mathrm{C}, n$-Hexane/i-Propanol $=70 / 30$ as eluent, $254 \mathrm{~nm}, 1 \mathrm{~mL} / \mathrm{min}$. $\mathrm{tR}=17$ $\min$ (major), 27 min (minor).

Optical Rotation: $[\alpha]_{D}{ }^{28}-4.4$ (c 5.0, MeCN) for $97 \%$ ee.

Absolute stereochemistry was determined through analogy with $\mathbf{3 a}$. 
$\overline{\mathrm{mAU}}$

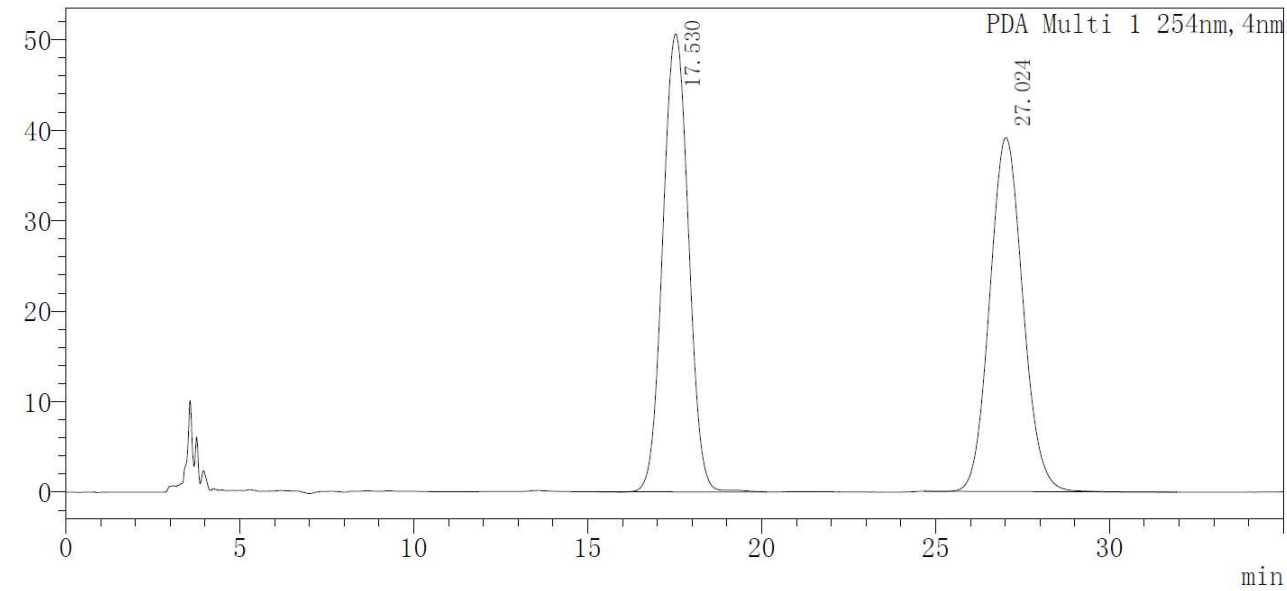

〈峰表〉

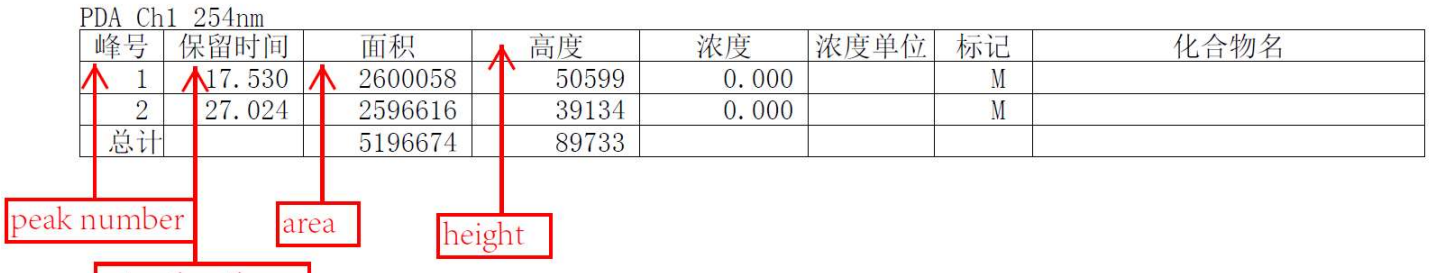

retention time

maU

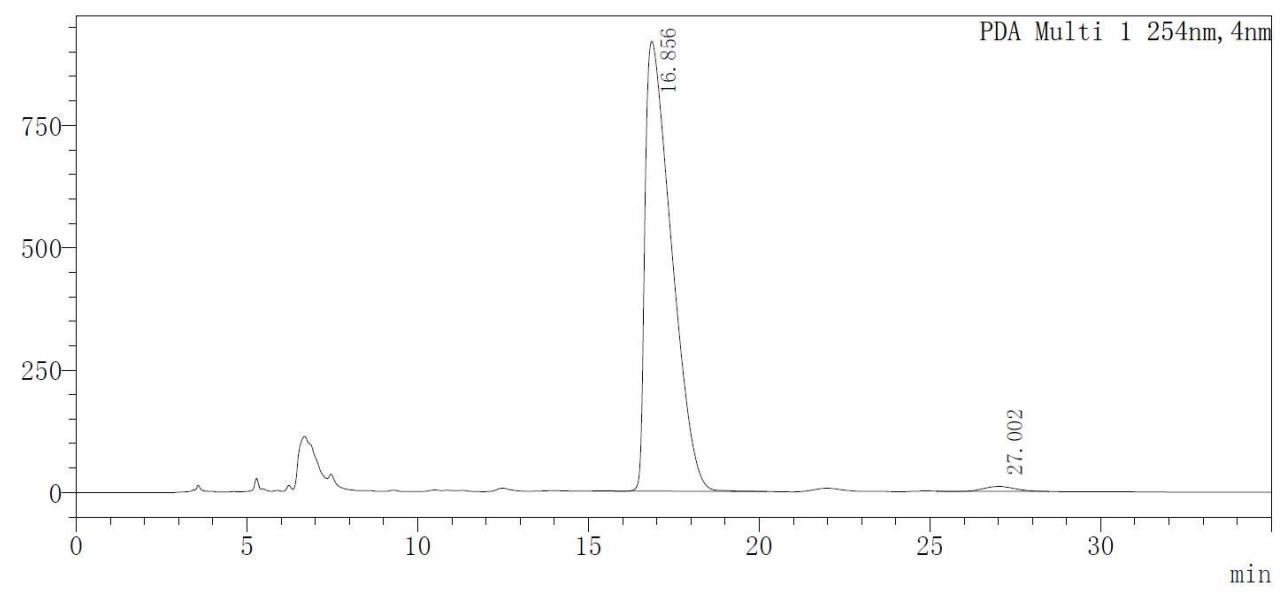

〈峰表〉

PDA Ch1 254nm

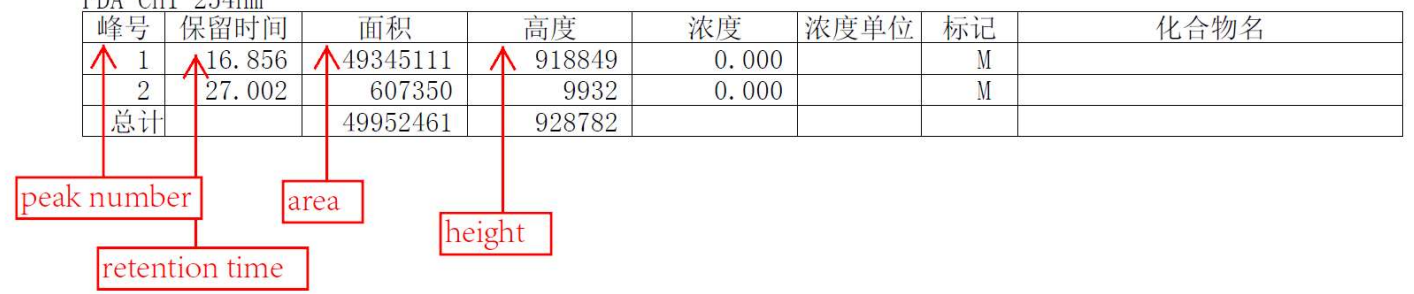




\section{4-fluoro-4',4'-dimethyl-2-phenyl-1'-tosylspiro[indene-1,3'-pyrrolidine]-3,5'(2H)-}

dione (3ai)

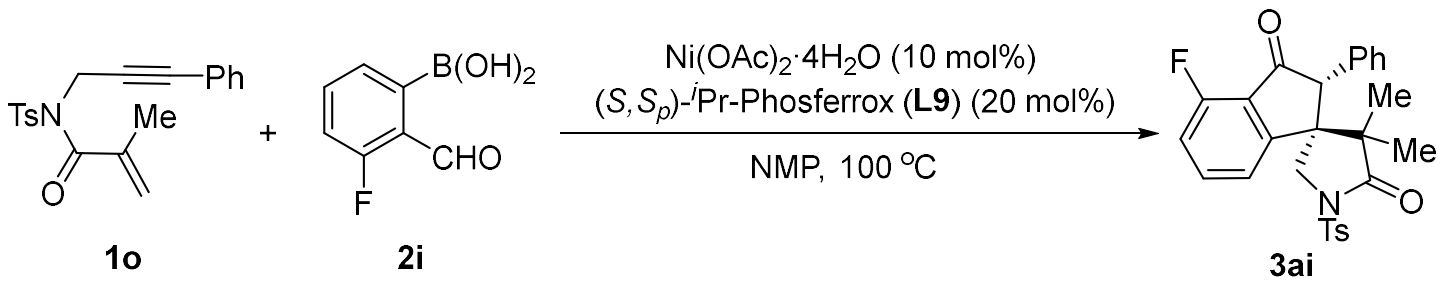

3ai was prepared according to general procedure using $10(0.1 \mathrm{mmol}, 35.3 \mathrm{mg})$ and 2i $(0.3 \mathrm{mmol}, 50.4 \mathrm{mg})$ and was purified by silica gel column chromatography (petroleum ether/ethyl acetate $=10 / 1 \sim 5 / 1)$ to obtain 3ai $(22.9 \mathrm{mg}, 48 \%$ yield).

${ }^{1} \mathrm{H}$ NMR $\left(600 \mathrm{MHz}, \mathrm{CDCl}_{3}\right) \delta 7.89(\mathrm{~d}, J=8.3 \mathrm{~Hz}, 2 \mathrm{H}), 7.45(\mathrm{td}, J=8.1,5.0 \mathrm{~Hz}, 1 \mathrm{H})$, 7.39-7.31 (m, 5H), $7.12(\mathrm{t}, J=8.5 \mathrm{~Hz}, 1 \mathrm{H}), 6.99-6.93(\mathrm{~m}, 2 \mathrm{H}), 6.91(\mathrm{~d}, J=7.7 \mathrm{~Hz}, 1 \mathrm{H})$, $3.85(\mathrm{~s}, 1 \mathrm{H}), 3.82(\mathrm{~d}, J=10.9 \mathrm{~Hz}, 1 \mathrm{H}), 3.50(\mathrm{~d}, J=11.0 \mathrm{~Hz}, 1 \mathrm{H}), 2.48(\mathrm{~s}, 3 \mathrm{H}), 1.22(\mathrm{~s}$, $3 \mathrm{H}), 0.71(\mathrm{~s}, 3 \mathrm{H})$;

${ }^{13} \mathrm{C}$ NMR $\left(151 \mathrm{MHz}, \mathrm{CDCl}_{3}\right) \delta 199.9,176.3,158.71$ (d, $\left.J=267.1 \mathrm{~Hz}\right), 158.4,145.6$, $138.1(\mathrm{~d}, J=8.4 \mathrm{~Hz}), 136.3,134.6,129.8,129.4,128.9,128.3,128.2,124.0(\mathrm{~d}, J=$ $12.9 \mathrm{~Hz}), 119.8(\mathrm{~d}, J=4.2 \mathrm{~Hz}), 116.4(\mathrm{~d}, J=18.8 \mathrm{~Hz}), 57.9,53.5,52.4,50.1,22.2$, 21.7, 18.6;

${ }^{19} \mathrm{~F}$ NMR $\left(376 \mathrm{MHz}, \mathrm{CDCl}_{3}\right) \delta-111.8(\mathrm{~m})$;

HRMS: (ESI) calcd for $\mathrm{C}_{27} \mathrm{H}_{25} \mathrm{FNO}_{4} \mathrm{~S}^{+}[\mathrm{M}+\mathrm{H}]^{+}$478.1483; found 478.1477 .

The enantiomeric purity was established by HPLC analysis using a chiral column: AD$\mathrm{H}$ column, $30^{\circ} \mathrm{C}, n$-Hexane/i-Propanol $=80 / 20$ as eluent, $254 \mathrm{~nm}, 1 \mathrm{~mL} / \mathrm{min} . \mathrm{tR}=14$ min (major), 20 min (minor).

Optical Rotation: $[\alpha]_{\mathrm{D}}^{28}-62.4$ (c 5.0, MeCN) for $91 \%$ ee.

Absolute stereochemistry was determined through analogy with $\mathbf{3 a}$. 
mAU

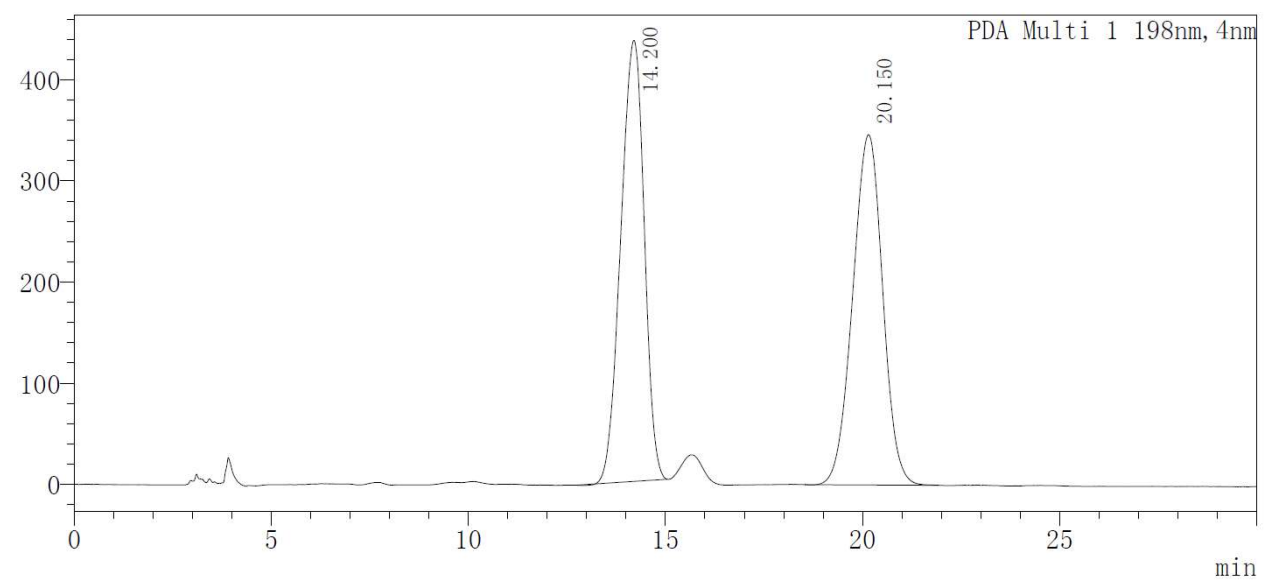

〈峰表〉
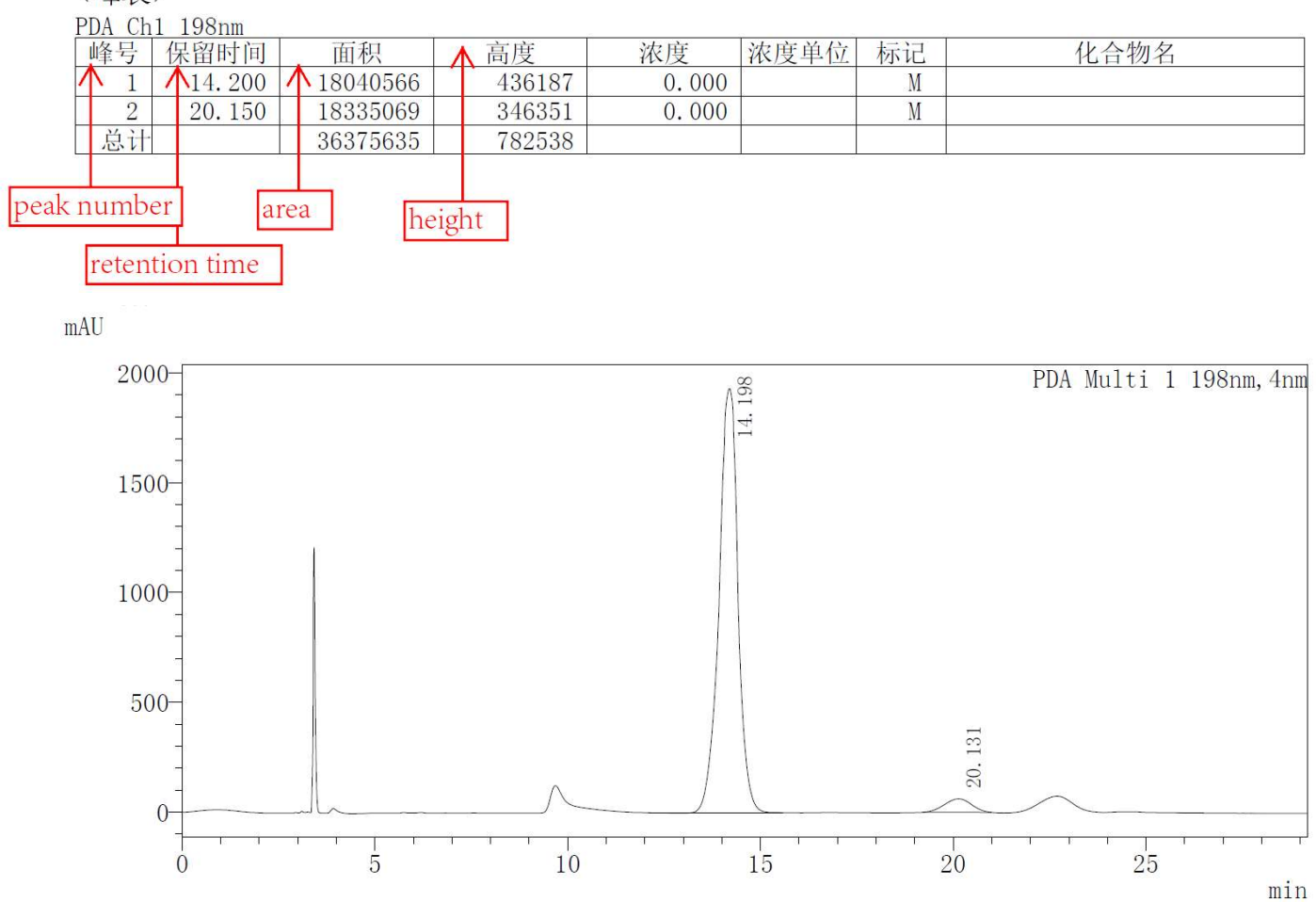

〈峰表〉

PDA Ch1 198nm

峰号 保留时间

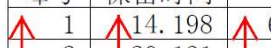

而和

\begin{tabular}{|l|l|l|}
\hline & 浓度单位 & 标记 \\
\hline
\end{tabular}

化合物名

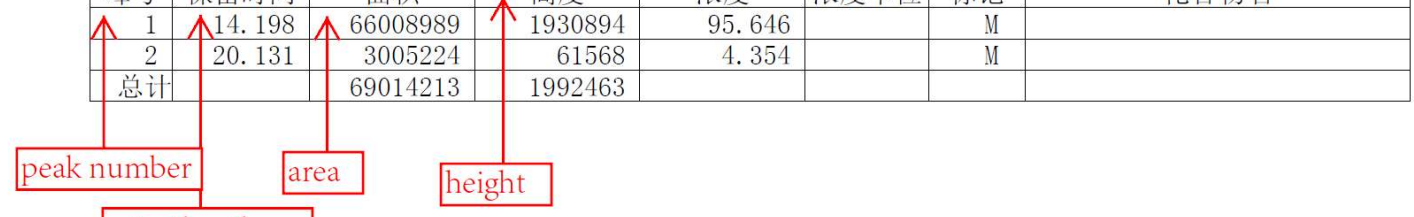

retention time 
4',4'-dimethyl-5-phenyl-1'-tosylspiro[cyclopenta[b]thiophene-4,3'-pyrrolidine]5',6(5H)-dione (3aj)

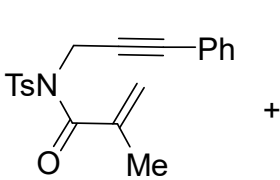

10<smiles>COc1ccsc1C=O</smiles>

2j

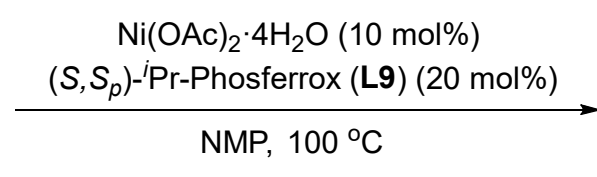

NMP, $100{ }^{\circ} \mathrm{C}$

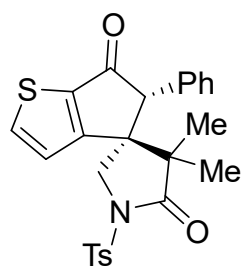

3aj

3aj was prepared according to general procedure using $10(0.1 \mathrm{mmol}, 35.3 \mathrm{mg})$ and 2j $(0.6 \mathrm{mmol}, 93.6 \mathrm{mg})$ and was purified by silica gel column chromatography (petroleum ether/ethyl acetate $=10 / 1 \sim 5 / 1)$ to obtain 3 aj $(18.6 \mathrm{mg}, 40 \%$ yield $)$.

${ }^{1} \mathrm{H}$ NMR $\left(600 \mathrm{MHz}, \mathrm{CDCl}_{3}\right)$ ס 7.89-7.87 (m, 3H), 7.38-7.32 (m, 5H), 7.06-7.04 (m, 2H), 6.62-6.61 (m, 1H), $4.10(\mathrm{~s}, 1 \mathrm{H}), 3.73(\mathrm{~d}, J=10.7 \mathrm{~Hz}, 1 \mathrm{H}), 3.37(\mathrm{~d}, J=10.7 \mathrm{~Hz}, 1 \mathrm{H})$, $2.46(\mathrm{~s}, 3 \mathrm{H}), 1.20(\mathrm{~s}, 3 \mathrm{H}), 0.78(\mathrm{~s}, 3 \mathrm{H})$;

${ }^{13} \mathrm{C}$ NMR $\left(151 \mathrm{MHz}, \mathrm{CDCl}_{3}\right) \delta 194.4,176.2,169.7,145.6,142.5,140.8,136.4,134.7$, $129.8,129.4,129.0,128.2,128.1,122.0,61.5,53.3,51.8,49.4,21.7,21.4,18.3$;

HRMS: (ESI) calcd for $\mathrm{C}_{25} \mathrm{H}_{24} \mathrm{NO}_{4} \mathrm{~S}_{2}{ }^{+}[\mathrm{M}+\mathrm{H}]^{+} 486.1141$; found 486.1138 .

The enantiomeric purity was established by HPLC analysis using a chiral column: OD$\mathrm{H}$ column, $30^{\circ} \mathrm{C}, n$-Hexane/i-Propanol $=80 / 20$ as eluent, $254 \mathrm{~nm}, 1 \mathrm{~mL} / \mathrm{min} . \mathrm{tR}=12$ min (major), 16 min (minor).

Optical Rotation: $[\alpha]_{D}^{28}-28.4$ (c 5.0, MeCN) for $67 \%$ ee.

Absolute stereochemistry was determined through analogy with $\mathbf{3 a}$. 
<Chromatogram>

mAU

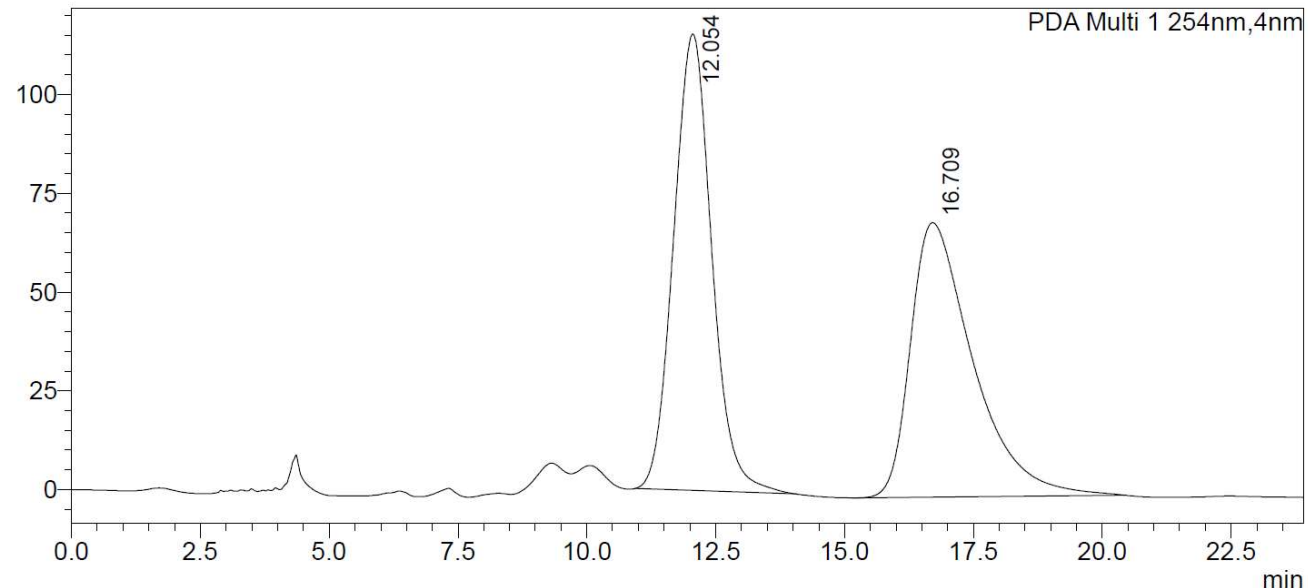

<Peak Table>

PDA Ch1 254nm

\begin{tabular}{|r|r|r|r|r|r|r|r|}
\hline Peak\# Ret. Time & \multicolumn{1}{|c|}{ Area } & Height & \multicolumn{1}{|c|}{ Conc. } & Unit & Mark & Name \\
\hline 1 & 12.054 & 5961911 & 115533 & 0.000 & & $\mathrm{M}$ & \\
\hline 2 & 16.709 & 5846578 & 69432 & 0.000 & & $\mathrm{M}$ & \\
\hline Total & & 11808489 & 184965 & & & & \\
\end{tabular}

<Chromatogram>

mAU

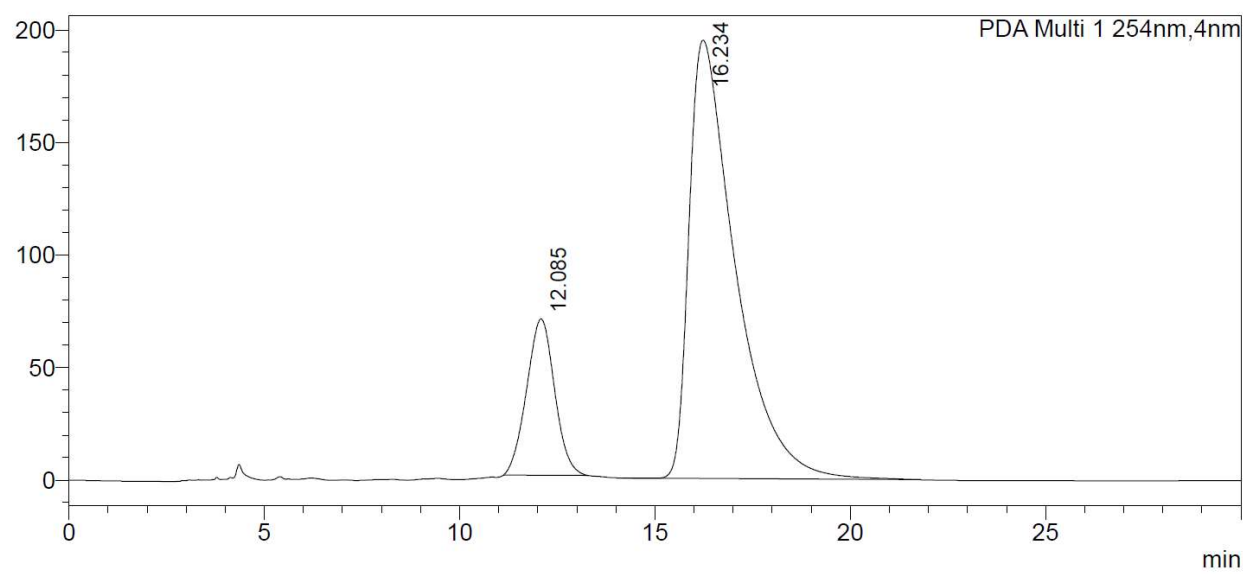

<Peak Table>

PDA Ch1 254nm

\begin{tabular}{|r|r|r|r|r|r|r|r|}
\hline Peak\# Ret. Time & \multicolumn{1}{|c|}{ Area } & Height & Conc. & Unit & Mark & Name \\
\hline 1 & 12.085 & 3462839 & 69460 & 0.000 & & $M$ & \\
\hline 2 & 16.234 & 16050766 & 194727 & 0.000 & & $M$ & \\
\hline Total & & 19513605 & 264186 & & & & \\
\hline
\end{tabular}




\section{$N$-((1-hydroxy-3-phenyl-1H-inden-2-yl)methyl)-4-methyl-N-(2-methylallyl)}

\section{benzenesulfonamide (4a)}

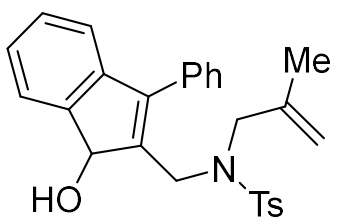

${ }^{1} \mathrm{H}$ NMR $\left(600 \mathrm{MHz}, \mathrm{CDCl}_{3}\right)$ ठ 7.73-7.67 (m, 2H), 7.64-7.58 (m, 1H), 7.45-7.33 (m, 3H), 7.31-7.24 (m, 6H), 7.14-7.06 (m, 1H), $5.45(\mathrm{~d}, J=4.7 \mathrm{~Hz}, 1 \mathrm{H}), 4.48(\mathrm{~s}, 1 \mathrm{H}), 4.44(\mathrm{~d}, J$ $=15.6 \mathrm{~Hz}, 1 \mathrm{H}), 4.28(\mathrm{~s}, 1 \mathrm{H}), 3.91(\mathrm{~d}, J=5.1 \mathrm{~Hz}, 1 \mathrm{H}), 3.85(\mathrm{~d}, J=15.6 \mathrm{~Hz}, 1 \mathrm{H}), 3.62$ (s, 2H), $2.41(\mathrm{~s}, 3 \mathrm{H}), 1.38(\mathrm{~s}, 3 \mathrm{H})$;

${ }^{13} \mathrm{C}$ NMR $\left(151 \mathrm{MHz}, \mathrm{CDCl}_{3}\right) \delta 144.3,143.6,143.2,142.9,140.6,139.0,136.5,133.4$, $129.7,128.8,128.5,128.2,128.0,127.2,126.6,124.1,120.4,114.6,75.4,54.4,42.8$, $21.5,19.8$.

HRMS: (ESI) calcd for $\mathrm{C}_{27} \mathrm{H}_{27} \mathrm{NO}_{3} \mathrm{NaS}^{+}[\mathrm{M}+\mathrm{Na}]^{+} 468.1604$; found 468.1600 . 
N-((1-hydroxy-2-phenyl-1H-inden-3-yl)methyl)-4-methyl-N-(2-

methylallyl)benzenesulfonamide (4a')

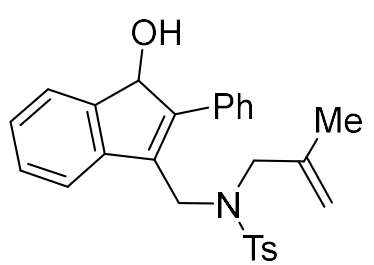

${ }^{1} \mathrm{H}$ NMR $\left(400 \mathrm{MHz}, \mathrm{CDCl}_{3}\right)$ ठ 7.81-7.75 (m, 1H), 7.69-7.62 (m, 2H), 7.56-7.51 (m, 1H), 7.44-7.37 (m, 2H), 7.36-7.31 (m, 2H), 7.30-7.24 (m, 5H), $5.38(\mathrm{~d}, J=7.1 \mathrm{~Hz}, 1 \mathrm{H}), 4.64$ (dd, J = 13.7, 1.0 Hz, 1H), 4.48 (s, 1H), $4.42(\mathrm{~s}, 1 \mathrm{H}), 4.17$ (d, J = 13.7 Hz, 1H), 3.52 (d, $J=15.6 \mathrm{~Hz}, 1 \mathrm{H}), 3.18(\mathrm{~d}, J=15.7 \mathrm{~Hz}, 1 \mathrm{H}), 2.43(\mathrm{~s}, 3 \mathrm{H}), 1.66(\mathrm{~d}, J=7.9 \mathrm{~Hz}, 1 \mathrm{H}), 1.32$ $(\mathrm{s}, 2 \mathrm{H})$;

${ }^{13} \mathrm{C} \mathrm{NMR}\left(101 \mathrm{MHz}, \mathrm{CDCl}_{3}\right) \delta 149.0,143.9,143.5,142.3,140.9,135.7,134.0,133.6$, $129.8,129.2,129.1,128.8,128.0,127.5,126.4,123.2,121.7,112.7,78.2,55.2,44.9$, 21.6, 20.0;

HRMS: (ESI) calcd for $\mathrm{C}_{27} \mathrm{H}_{27} \mathrm{NO}_{3} \mathrm{NaS}^{+}[\mathrm{M}+\mathrm{Na}]^{+} 468.1604$; found 468.1592. 
N-((2-((benzyloxy)methyl)-1-hydroxy-1H-inden-3-yl)methyl)-4-methyl-N-(2methylallyl)benzenesulfonamide ( $\left.4 \mathrm{~m}^{\prime}\right)$<smiles>C=C(C)CN(C)CC1=C(COCc2ccccc2)C(O)c2ccccc21</smiles>

${ }^{1} \mathrm{H}$ NMR $\left(600 \mathrm{MHz} \mathrm{CDCl}_{3}\right) \delta$ 7.74-7.68 (m, 2H), $7.50(\mathrm{~d}, J=7.2 \mathrm{~Hz}, 1 \mathrm{H}), 7.35-7.26(\mathrm{~m}$, $9 \mathrm{H}), 7.25-7.21(\mathrm{~m}, 1 \mathrm{H}), 5.13(\mathrm{~s}, 1 \mathrm{H}), 4.80(\mathrm{t}, J=1.5 \mathrm{~Hz}, 1 \mathrm{H}), 4.73(\mathrm{~s}, 1 \mathrm{H}), 4.53(\mathrm{~d}, J=$ $11.8 \mathrm{~Hz}, 1 \mathrm{H}), 4.50$ (d, $J=11.8 \mathrm{~Hz}, 1 \mathrm{H}), 4.40$ (d, $J=12.1 \mathrm{~Hz}, 1 \mathrm{H}), 4.36$ (d, $J=12.3 \mathrm{~Hz}$, 1H), 4.33 (d, J = $15.5 \mathrm{~Hz}, 1 \mathrm{H}), 3.96(\mathrm{~d}, J=15.4 \mathrm{~Hz}, 1 \mathrm{H}), 3.78(\mathrm{~d}, J=15.4 \mathrm{~Hz}, 1 \mathrm{H})$, 3.75 (d, $J=15.5 \mathrm{~Hz}, 1 \mathrm{H}), 3.42(\mathrm{~d}, J=5.7 \mathrm{~Hz}, 1 \mathrm{H}), 2.41$ (s, 3H), 1.51 (s, 3H);

${ }^{13} \mathrm{C}$ NMR $\left(151 \mathrm{MHz}, \mathrm{CDCl}_{3}\right) \delta 144.1,143.6,142.5,141.8,140.1,138.4,137.8,136.8$, $129.7,128.4,127.9,127.8,127.2,126.5,123.8,120.4,114.3,75.6,72.8,63.6,54.1$, 42.1, 21.5, 19.9;

HRMS: (ESI) calcd for $\mathrm{C}_{29} \mathrm{H}_{32} \mathrm{NO}_{4} \mathrm{~S}^{+}[\mathrm{M}+\mathrm{H}]^{+} 490.2047$; found 490.2038 . 


\section{Gram-scale reaction and synthetic transformations}

\section{Gram-scale reaction:}

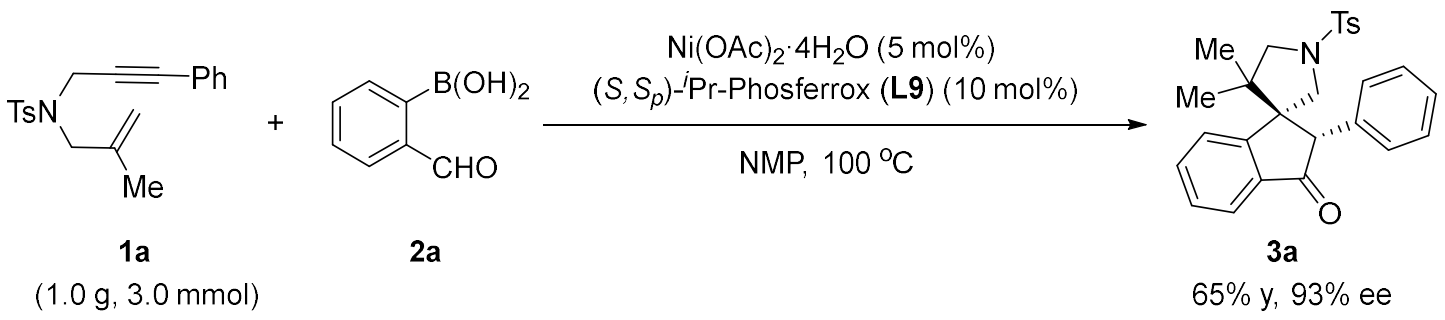

An oven-dried sealed tube equipped with a PTFE-coated stir bar was charged with $\mathrm{Ni}(\mathrm{OAc})_{2} \cdot 4 \mathrm{H}_{2} \mathrm{O}$ (0.15 mmol, $\left.37.5 \mathrm{mg}\right),(\mathrm{S}, \mathrm{Sp}){ }^{i}{ }^{-} \mathrm{Pr}-\mathrm{Phosferrox}$ (L9) $(0.3 \mathrm{mmol}, 144.0 \mathrm{mg})$, (2-formylphenyl)boronic acid 2a (9.0 mol, $1.35 \mathrm{~g})$ and $1 \mathrm{a}(3.0 \mathrm{mmol}, 1.0 \mathrm{~g})$. The sealed tube was evacuated and backfilled with argon (this process was repeated for three times) and then NMP $(20 \mathrm{~mL})$ was added. This reaction mixture was stirred at room temperature for 15 minutes and then heated at $100{ }^{\circ} \mathrm{C}$ for 36 hours until the reaction was complete. The resulting mixture was quenched with sat. $\mathrm{NH}_{4} \mathrm{Cl}$ solution $(20 \mathrm{~mL})$ and further diluted with water $(20 \mathrm{~mL})$. The aqueous layer was extracted with EtOAc $(3 \times 25 \mathrm{~mL})$ and the combined organic layers were washed with brine $(2 \times 30 \mathrm{~mL})$, dried with $\mathrm{MgSO}_{4}$, filtered, and concentrated under reduced pressure. The residue was purified by chromatography on silica gel, eluting with ethyl acetate/petroleum ether 1:10 1:5 (v/v) to afford $3 \mathrm{a}(0.87 \mathrm{~g}, 65 \%$ yield, $93 \% \mathrm{ee})$. 
<smiles>CC1(C)CN([13F])C[C@@]12c1ccccc1C(=O)[C@H]2c1ccccc1</smiles>

$3 a, 92 \%$ ee

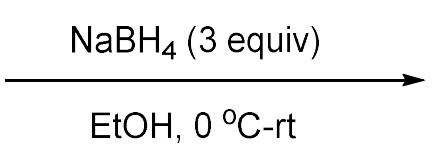

$\mathbf{5}, 90 \%$ y, 10/1 d.r.

To a solution of $3 a(44.5 \mathrm{mg}, 0.1 \mathrm{mmol})$ in $\mathrm{EtOH}(2 \mathrm{~mL})$ were added $\mathrm{NaBH}_{4}(10.0 \mathrm{mg}$, $0.3 \mathrm{mmol}$ ) at $0{ }^{\circ} \mathrm{C}$. The reaction mixture was then stirred for 2 hours at room temperature. The reaction mixture was quenched with saturated $\mathrm{NH}_{4} \mathrm{Cl}$ aqueous solution and extracted with EtOAc. The combined organic layers were washed with brine, dried over anhydrous $\mathrm{Na}_{2} \mathrm{SO}_{4}$ and filtered. The solvent was concentrated under reduced pressure and purified by silica-gel column chromatography, eluting with petroleum ether/ethyl acetate 10/1 3/1 (v/v) to provide the alcohol $5(40.2 \mathrm{mg}, 90 \%$, 10/1 d.r.).

${ }^{1} \mathrm{H}$ NMR $\left(600 \mathrm{MHz}, \mathrm{CDCl}_{3}\right) \delta 7.65(\mathrm{~d}, J=8.2 \mathrm{~Hz}, 2 \mathrm{H}), 7.41$ (d, $\left.J=7.3 \mathrm{~Hz}, 1 \mathrm{H}\right), 7.36-$ $7.28(\mathrm{~m}, 4 \mathrm{H}), 7.25-7.21(\mathrm{~m}, 1 \mathrm{H}), 7.20-7.16(\mathrm{~m}, 1 \mathrm{H}), 7.11-7.02(\mathrm{~m}, 2 \mathrm{H}), 6.84(\mathrm{~d}, J=7.6$ $\mathrm{Hz}, 1 \mathrm{H}), 6.33-6.25(\mathrm{~m}, 1 \mathrm{H}), 5.51-5.44(\mathrm{~m}, 1 \mathrm{H}), 3.83(\mathrm{~d}, J=7.3 \mathrm{~Hz}, 1 \mathrm{H}), 3.34(\mathrm{~d}, J=$ 10.0 Hz, 1H), 3.24 (dd, J = 10.3, 8.0 Hz, 2H), 3.06 (d, $J=10.6 \mathrm{~Hz}, 1 \mathrm{H}), 2.47(\mathrm{~s}, 3 \mathrm{H}$ ), $0.99(\mathrm{~s}, 3 \mathrm{H}), 0.58(\mathrm{~s}, 3 \mathrm{H})$;

${ }^{13} \mathrm{C} \mathrm{NMR}\left(151 \mathrm{MHz}, \mathrm{CDCl}_{3}\right) \delta 145.1,144.7,143.4,136.6,134.6,132.1,129.7,129.2$, $128.7,128.2,127.9,127.2,125.7,123.9,123.6,75.7,61.8,59.5,57.2,56.0,44.5,24.9$, 21.6, 21.5;

HRMS: (ESI) calcd for $\mathrm{C}_{27} \mathrm{H}_{30} \mathrm{NO}_{3} \mathrm{~S}^{+}[\mathrm{M}+\mathrm{H}]^{+}$448.1941; found 448.1943.

Optical Rotation: $[\alpha]_{D}^{28}-18.2$ (c 2.0, MeCN). 


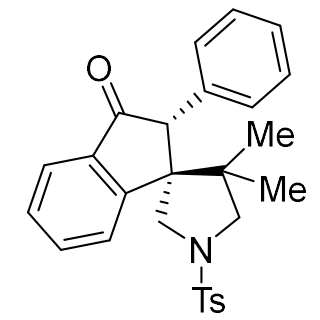

$3 a, 92 \%$ ee

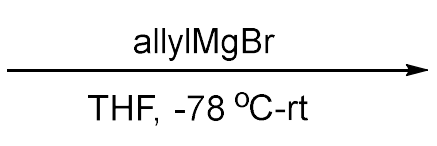

$6,96 \%$ y: $>20 / 1$ d.r.

The reaction was conducted following literature procedure. ${ }^{2}$ To a solution of $3 \mathbf{a}(44.5$ $\mathrm{mg}, 0.1 \mathrm{mmol})$ in anhydrous THF $(2 \mathrm{~mL})$ was added allylmagnesium bromide $(1 \mathrm{~mol} / \mathrm{L}$ in $\mathrm{Et}_{2} \mathrm{O}, 0.3 \mathrm{~mL}, 0.3 \mathrm{mmol}$ ) at $-78^{\circ} \mathrm{C}$. The reaction mixture was allowed to warm up to room temperature and was stirred for 24 hours. Then, the reaction mixture was quenched with saturated $\mathrm{NH}_{4} \mathrm{Cl}$ aqueous solution and extracted with EtOAc. The combined organic layers were washed with brine, dried over anhydrous $\mathrm{Na}_{2} \mathrm{SO}_{4}$ and filtered. The solvent was concentrated under reduced pressure and purified by silicagel column chromatography, eluting with petroleum ether/ethyl acetate 10/1 3/1 (v/v) to provide the allyl alcohol $6(46.8 \mathrm{mg}, 96 \%$, d.r. $>20 / 1)$.

${ }^{1} \mathrm{H} \mathrm{NMR}\left(600 \mathrm{MHz}, \mathrm{CDCl}_{3}\right) \delta 7.63(\mathrm{~d}, J=8.2 \mathrm{~Hz}, 2 \mathrm{H}), 7.41(\mathrm{~d}, J=7.6 \mathrm{~Hz}, 1 \mathrm{H}), 7.35-$ 7.26 (m, 4H), 7.25-7.17 (m, 3H), 7.13-7.04 (m, 3H), 5.76-5.67 (m, 1H), 5.20-5.10 (m, 2H), $3.63(\mathrm{~s}, 1 \mathrm{H}), 3.55(\mathrm{dd}, J=14.0,10.1 \mathrm{~Hz}, 2 \mathrm{H}), 3.37(\mathrm{~d}, J=9.7 \mathrm{~Hz}, 1 \mathrm{H}), 3.15(\mathrm{~d}, J$ $=10.4 \mathrm{~Hz}, 1 \mathrm{H}), 2.72-2.64(\mathrm{~m}, 1 \mathrm{H}), 2.55-2.47(\mathrm{~m}, 1 \mathrm{H}), 2.40(\mathrm{~s}, 3 \mathrm{H}), 0.69(\mathrm{~s}, 3 \mathrm{H}), 0.62$ $(\mathrm{s}, 3 \mathrm{H})$;

${ }^{13} \mathrm{C}$ NMR $\left(151 \mathrm{MHz}, \mathrm{CDCl}_{3}\right) \delta$ 146.5, 145.0, 143.3, 137.6, 134.2, 129.5, 129.3, 128.0, 127.2, 127.1, 125.2, 123.6, 119.1, 81.5, 61.2, 59.8, 56.8, 56.3, 45.7, 45.1, 26.7, 21.5, 21.4 ;

HRMS: (ESI) calcd for $\mathrm{C}_{30} \mathrm{H}_{34} \mathrm{NO}_{3} \mathrm{~S}^{+}[\mathrm{M}+\mathrm{H}]^{+} 488.2254$; found 488.2251 .

Optical Rotation: $[\alpha]_{D}^{28}-35.9$ (c 4.0, MeCN). 
(1R,2S)-4',4'-dimethyl-2-phenylspiro[indene-1,3'-pyrrolidin]-3(2H)-one (7)

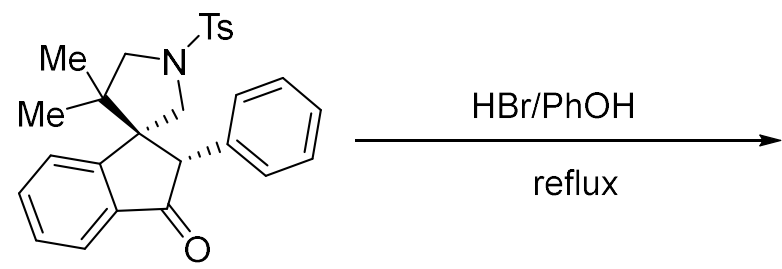

$3 a, 93 \%$ ee

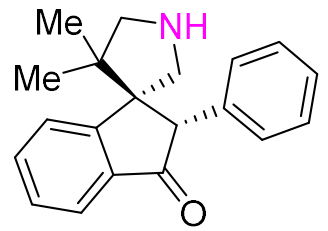

$7,70 \%$

The reaction was conducted following literature procedure. ${ }^{3}$ To a solution of $\mathbf{3 a}$ (44.5 $\mathrm{mg}, 0.1 \mathrm{mmol}, 93 \% \mathrm{ee})$ was added phenol $(0.10 \mathrm{~g})$ and $33 \% \mathrm{HBr}$ in $\mathrm{HOAc}(1 \mathrm{~mL})$. The mixture was heated to $120{ }^{\circ} \mathrm{C}$ for $2 \mathrm{~h}$. The acidic mixture was cooled to room temperature and was washed with diethyl ether. It was made alkaline with $30 \%$ sodium hydroxide solution and extracted with diethyl ether. The combined diethyl ether extracts of the alkaline solution were dried over $\mathrm{Na}_{2} \mathrm{SO}_{4}$ and filtered. The solvent was concentrated under reduced pressure to provide the allyl alcohol $7(20.4 \mathrm{mg}, 70 \%)$.

${ }^{1} \mathrm{H}$ NMR $\left(600 \mathrm{MHz}, \mathrm{CDCl}_{3}\right) \delta 7.85(\mathrm{~d}, J=7.6 \mathrm{~Hz}, 1 \mathrm{H}), 7.71$ (d, $\left.J=3.6 \mathrm{~Hz}, 2 \mathrm{H}\right), 7.52-$ $7.47(\mathrm{~m}, 1 \mathrm{H}), 7.25-7.16(\mathrm{~m}, 3 \mathrm{H}), 6.88-6.83(\mathrm{~m}, 2 \mathrm{H}), 3.91(\mathrm{~s}, 1 \mathrm{H}), 3.20(\mathrm{~d}, J=11.0 \mathrm{~Hz}$, 1H), $3.10(\mathrm{~d}, J=11.6 \mathrm{~Hz}, 1 \mathrm{H}), 3.05(\mathrm{~d}, J=11.0 \mathrm{~Hz}, 1 \mathrm{H}), 2.96(\mathrm{~d}, J=11.6 \mathrm{~Hz}, 1 \mathrm{H}), 1.20$ (s, 3H), 0.65 (s, 3H);

${ }^{13} \mathrm{C}$ NMR $\left(151 \mathrm{MHz}, \mathrm{CDCl}_{3}\right) \delta 205.7,158.3,138.8,136.9,135.4,128.8,128.5,128.4$, $127.4,126.4,123.9,60.0,60.0,58.5,52.9,45.8,24.9,22.4$;

HRMS: (ESI) calcd for $\mathrm{C}_{20} \mathrm{H}_{22} \mathrm{NO}[\mathrm{M}+\mathrm{H}]^{+}$292.1696; found 292.1709.

Optical Rotation: $[\alpha]_{D}^{28}-58.8$ (c 1.0, MeCN). 
$N^{\prime}-((1 R, 2 S, E)-4$ ',4'-dimethyl-2-phenyl-1'-tosylspiro[indene-1,3'-pyrrolidin]-3(2H)ylidene)-4-methylbenzenesulfonohydrazide (8)

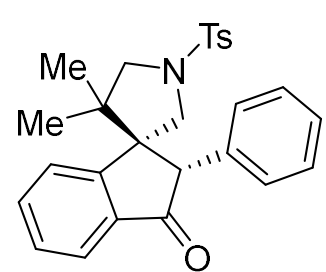

$3 a, 93 \%$ ee

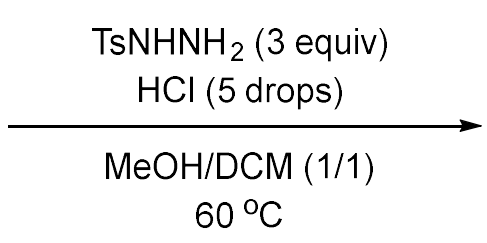

$60^{\circ} \mathrm{C}$

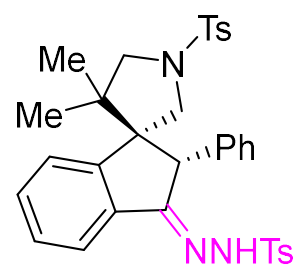

$8,68 \%$

A solution of 4-methylbenzenesulfon hydrazide $(558.0 \mathrm{mg}, 2 \mathrm{mmol})$ in dry $\mathrm{MeOH}(5$ $\mathrm{mL}$ ) was stirred at $60{ }^{\circ} \mathrm{C}$ until the hydrazide was completely dissolved. The mixture was cooled to room temperature. Then $3 \mathrm{a}(445.2 \mathrm{mg}, 1 \mathrm{mmol})$ and $\mathrm{DCM}(5 \mathrm{~mL})$ were added dropwise to the mixture. The solvent was then concentrated under reduced pressure and purified by silica-gel column chromatography, eluting with petroleum ether/ethyl acetate 10/1 3/1 (v/v) to provide the $N$-tosylhydrazone 8 (416.8 $\mathrm{mg}, 68 \%)$. ${ }^{1} \mathrm{H} \mathrm{NMR}\left(600 \mathrm{MHz}, \mathrm{CDCl}_{3}\right) \delta 7.72(\mathrm{~d}, J=7.0 \mathrm{~Hz}, 1 \mathrm{H}), 7.58(\mathrm{~d}, J=8.2 \mathrm{~Hz}, 2 \mathrm{H}), 7.39$ (d, $J=8.3 \mathrm{~Hz}, 2 \mathrm{H}), 7.35-7.32(\mathrm{~m}, 1 \mathrm{H}), 7.31-7.27(\mathrm{~m}, 4 \mathrm{H}), 7.25(\mathrm{~d}, J=7.4 \mathrm{~Hz}, 1 \mathrm{H}), 7.14$ (d, $J=8.0 \mathrm{~Hz}, 2 \mathrm{H}), 7.12-7.03(\mathrm{~m}, 3 \mathrm{H}), 6.69(\mathrm{~s}, 2 \mathrm{H}), 3.98(\mathrm{~s}, 1 \mathrm{H}), 3.39$ (d, J = $10.5 \mathrm{~Hz}$, 1H), 3.27 (d, $J=10.4 \mathrm{~Hz}, 1 \mathrm{H}), 3.07$ (d, $J=10.3 \mathrm{~Hz}, 1 \mathrm{H}), 2.83(\mathrm{~d}, J=10.3 \mathrm{~Hz}, 1 \mathrm{H}$ ), $2.44(\mathrm{~s}, 3 \mathrm{H}), 2.36(\mathrm{~s}, 3 \mathrm{H}), 0.78(\mathrm{~s}, 3 \mathrm{H}), 0.39(\mathrm{~s}, 3 \mathrm{H})$;

${ }^{13} \mathrm{C} \mathrm{NMR}\left(151 \mathrm{MHz}, \mathrm{CDCl}_{3}\right) \delta 160.6,149.5,143.8,143.6,136.9,135.5,134.8,134.2$, 131.6, 129.7, 129.6, 129.3, 128.5, 128.3, 127.5, 127.0, 125.1, 121.7, 61.6, 59.2, 53.6, $49.8,44.9,23.8,21.5,21.5,21.3$;

HRMS: (ESI) calcd for $\mathrm{C}_{34} \mathrm{H}_{36} \mathrm{~N}_{3} \mathrm{O}_{4} \mathrm{~S}_{2}{ }^{+}[\mathrm{M}+\mathrm{H}]^{+}$614.2142; found 614.2154.

Optical Rotation: $[\alpha]_{D}^{28}-207.7$ (c $0.3, \mathrm{MeCN}$ ). 
(S)-4',4'-dimethyl-2-phenyl-3-(phenylethynyl)-1'-tosylspiro[indene-1,3'-

pyrrolidine] (9)

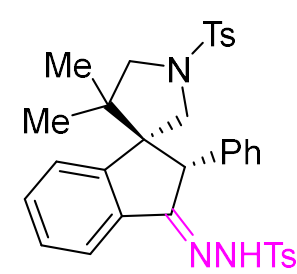

8<smiles>C#Cc1ccccc1</smiles>

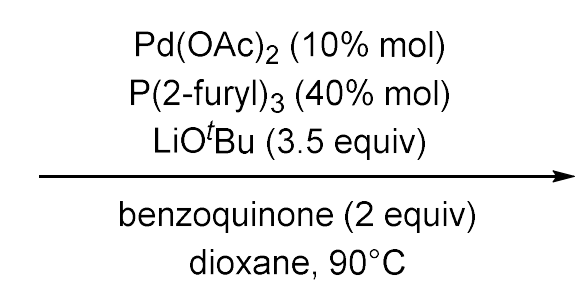

dioxane, $90^{\circ} \mathrm{C}$

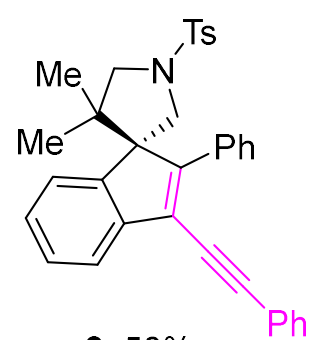

$9,58 \%$

The reaction was conducted following literature procedure. ${ }^{4} \mathrm{Pd}(\mathrm{OAc})_{2}(0.01 \mathrm{mmol}, 2.3$ $\mathrm{mg}$ ), tri-(2-furyl)phosphine (0.02 mmol, $4.6 \mathrm{mg})$, ${ }^{t} \mathrm{BuOLi}(3.5$ equiv, $24.0 \mathrm{mg}$ ), benzoquinone (2 equiv, $31.6 \mathrm{mg}$ ) and $N$-tosylhydrazone 8 ( $0.1 \mathrm{mmol}, 61.3 \mathrm{mg}$ ) were suspended in 1,4-dioxane $(2 \mathrm{~mL})$ in a $5 \mathrm{~mL}$ sealed tube under argon. Then phenyl acetylene (1.5 equiv, $15.3 \mathrm{mg}$ ) was added. The resulting solution was stirred at $90{ }^{\circ} \mathrm{C}$ for $4 \mathrm{~h}$. The solvent was then evaporated under reduced pressure and purified by silicagel column chromatographyto provide the alkene 9 (30.7 $\mathrm{mg}, 58 \%)$.

${ }^{1} \mathrm{H}$ NMR (400 MHz, $\left.\mathrm{CDCl}_{3}\right) \delta 7.80(\mathrm{~d}, J=8.2 \mathrm{~Hz}, 2 \mathrm{H}), 7.56(\mathrm{~d}, J=7.4 \mathrm{~Hz}, 1 \mathrm{H}), 7.55-$ $7.50(\mathrm{~m}, 2 \mathrm{H})$, 7.41-7.34 (m, 8H), 7.32-7.27 (m, 3H), 7.20-7.14 (m, 2H), 4.21 (d, J = 9.9 Hz, 1H), 3.91 (d, J = 9.9 Hz, 1H), 3.35 (d, $J=10.3 \mathrm{~Hz}, 1 \mathrm{H}), 2.97(\mathrm{~d}, J=10.3 \mathrm{~Hz}, 1 \mathrm{H})$, $2.47(\mathrm{~s}, 3 \mathrm{H}), 0.69(\mathrm{~s}, 3 \mathrm{H}), 0.58(\mathrm{~s}, 3 \mathrm{H})$;

${ }^{13} \mathrm{C}$ NMR $\left(151 \mathrm{MHz}, \mathrm{CDCl}_{3}\right) \delta 153.8,143.9,143.5,142.5,137.0,135.2,131.7,129.7$, $129.1,128.5,128.3,128.1,128.0,127.9,127.2,125.9,125.5,123.9,123.0,120.7$, $95.6,83.4,66.9,59.8,52.3,45.7,24.9,22.7,21.6$

HRMS: (ESI) calcd for $\mathrm{C}_{35} \mathrm{H}_{32} \mathrm{NO}_{2} \mathrm{~S}^{+}[\mathrm{M}+\mathrm{H}]^{+} 530.2148$; found 530.2145 .

Optical Rotation: $[\alpha]_{D}^{28} 65.5$ (c 1.0, MeCN). 


\section{(S)-3-(4-methoxyphenyl)-4',4'-dimethyl-2-phenyl-1'-tosylspiro[indene-1,3'-}

\section{pyrrolidine] (10)}

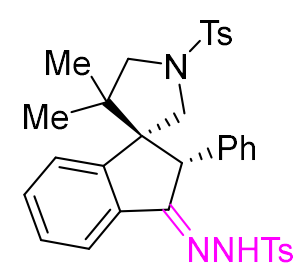

8<smiles>COc1ccc(I)cc1</smiles>

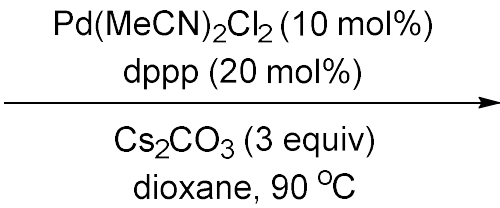

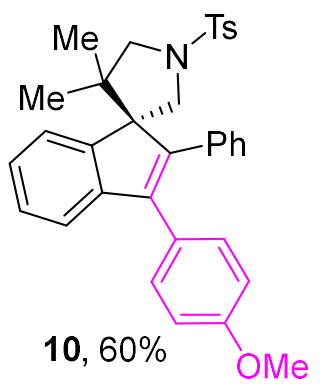

The reaction was conducted following literature procedure. ${ }^{5}$ Tosylhydrazone 8 (0.1 mmol, $61.3 \mathrm{mg}), \mathrm{PdCl}_{2}(\mathrm{MeCN})_{2}(0.01 \mathrm{mmol}, 2.6 \mathrm{mg})$, dppp (0.02 mmol, $\left.8.2 \mathrm{mg}\right)$, $\mathrm{Cs}_{2} \mathrm{CO}_{3}(3.0 \mathrm{mmol}, 97.1 \mathrm{mg})$ and dioxane $(5 \mathrm{~mL})$ were mixed in an oven dried sealed tube under argon for 5 minutes at room temperature. 1-lodo-4-methoxybenzene (1.0 mmol, $23.3 \mathrm{mg}$ ) was then added, the mixture was stirred at $90{ }^{\circ} \mathrm{C}$ until the reaction completed by TLC analysis. The solvent was then evaporated under reduced pressure and purified by silica-gel column chromatographyto provide the alkene 10 (32.1 mg, $60 \%)$.

${ }^{1} \mathrm{H}$ NMR $\left(600 \mathrm{MHz}, \mathrm{CDCl}_{3}\right) \delta 7.79(\mathrm{~d}, J=8.3 \mathrm{~Hz}, 2 \mathrm{H}), 7.35(\mathrm{~d}, J=8.0 \mathrm{~Hz}, 2 \mathrm{H}), 7.25-$ $7.14(\mathrm{~m}, 6 \mathrm{H}), 7.14-7.08(\mathrm{~m}, 3 \mathrm{H}), 7.00(\mathrm{~d}, \mathrm{~J}=8.1 \mathrm{~Hz}, 2 \mathrm{H}), 6.77(\mathrm{~d}, \mathrm{~J}=9.0 \mathrm{~Hz}, 2 \mathrm{H}), 4.24$ (d, $J=9.8 \mathrm{~Hz}, 1 \mathrm{H}), 3.91(\mathrm{~d}, J=9.8 \mathrm{~Hz}, 1 \mathrm{H}), 3.77(\mathrm{~s}, 3 \mathrm{H}), 3.36(\mathrm{~d}, J=10.1 \mathrm{~Hz}, 1 \mathrm{H})$, $2.92(\mathrm{~d}, J=10.2 \mathrm{~Hz}, 1 \mathrm{H}), 2.46(\mathrm{~s}, 3 \mathrm{H}), 0.73(\mathrm{~s}, 3 \mathrm{H}), 0.61(\mathrm{~s}, 3 \mathrm{H})$;

${ }^{13} \mathrm{C}$ NMR $\left(151 \mathrm{MHz}, \mathrm{CDCl}_{3}\right) \delta 158.5,145.3,145.1,144.4,143.4,142.9,137.4,135.3$, $130.7,130.2,129.7,127.9,127.4,127.2,127.0,126.7,125.3,123.9,120.7,113.5$, $66.4,60.1,55.1,52.4,45.3,24.8,22.9,21.6$

HRMS: (ESI) calcd for $\mathrm{C}_{34} \mathrm{H}_{34} \mathrm{NO}_{3} \mathrm{~S}^{+}[\mathrm{M}+\mathrm{H}]^{+} 536.2254$; found 536.2252.

Optical Rotation: $[\alpha]_{\mathrm{D}}^{28} 299.9$ (c 1.4, MeCN). 
6. Single crystal X-ray diffraction

CCDC: 2022309 (3a)

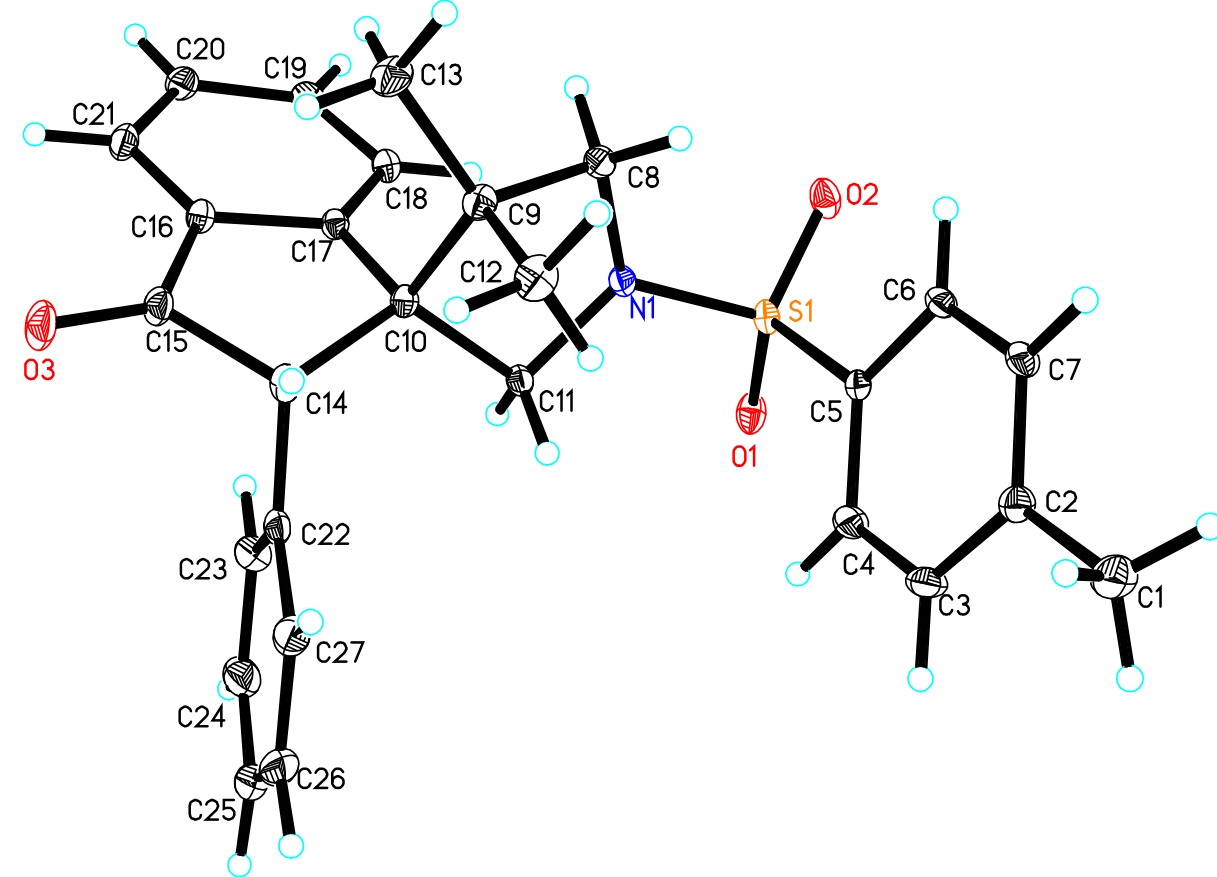


Table 1. Crystal data and structure refinement for $\mathbf{3 a}$.

Identification code

Empirical formula

Formula weight

Temperature

Wavelength

Crystal system

Space group

Unit cell dimensions

Volume

Z

Density (calculated)

Absorption coefficient

$\mathrm{F}(000)$

Crystal size

Theta range for data collection

Index ranges

Reflections collected

Independent reflections

Completeness to theta $=25.242^{\circ}$

Absorption correction

Max. and min. transmission

Refinement method

Data / restraints / parameters

Goodness-of-fit on $\mathrm{F}^{2}$

Final $R$ indices [ $\mid>2$ sigma $(I)]$

$\mathrm{R}$ indices (all data)

Absolute structure parameter

Extinction coefficient

Largest diff. peak and hole mo_200107b_0m

$\mathrm{C} 27 \mathrm{H} 27 \mathrm{~N} \mathrm{O} 3 \mathrm{~S}$

445.55

296(2) K

$0.71073 \AA$

Monoclinic

C 121

$a=18.8079(11) \AA$

$a=90^{\circ}$.

$b=8.1557(5) \AA$

$b=107.103(2)^{\circ}$.

$c=16.0455(10) \AA$

$\mathrm{g}=90^{\circ}$.
4

$1.258 \mathrm{Mg} / \mathrm{m}^{3}$

$0.166 \mathrm{~mm}^{-1}$

944

$0.12 \times 0.1 \times 0.1 \mathrm{~mm}^{3}$

1.328 to $27.965^{\circ}$.

$-24<=\mathrm{h}<=22,-10<=\mathrm{k}<=10,-20<=\mid<=18$

12583

$4979[R($ int $)=0.0488]$

$100.0 \%$

Semi-empirical from equivalents

0.7456 and 0.4807

Full-matrix least-squares on $\mathrm{F}^{2}$

4979 / 1 / 292

1.078

$\mathrm{R} 1=0.0430, w R 2=0.1045$

$\mathrm{R} 1=0.0520, w R 2=0.1108$

$-0.01(5)$

$\mathrm{n} / \mathrm{a}$

0.226 and -0.199 e. $\AA^{-3}$ 
Table 2. Atomic coordinates $\left(x 10^{4}\right)$ and equivalent isotropic displacement parameters $\left(\AA^{2} x\right.$ $10^{3}$ ) for $3 a$. $U(e q)$ is defined as one third of the trace of the orthogonalized Uij tensor.

\begin{tabular}{|c|c|c|c|c|}
\hline & $x$ & $y$ & $z$ & $\mathrm{U}(\mathrm{eq})$ \\
\hline$C(1)$ & $232(3)$ & $-4869(5)$ & $1344(3)$ & $74(1)$ \\
\hline$C(2)$ & $612(2)$ & $-3343(4)$ & 1151(2) & $52(1)$ \\
\hline$C(3)$ & $371(2)$ & $-1797(5)$ & $1304(3)$ & $62(1)$ \\
\hline$C(4)$ & $700(2)$ & $-384(5)$ & 1116(3) & $55(1)$ \\
\hline$C(5)$ & 1295(2) & $-510(4)$ & $776(2)$ & $38(1)$ \\
\hline$C(6)$ & $1550(2)$ & $-2044(4)$ & $625(2)$ & $44(1)$ \\
\hline$C(7)$ & $1204(2)$ & $-3441(4)$ & $809(2)$ & $48(1)$ \\
\hline$C(8)$ & $3067(2)$ & $829(4)$ & $1741(2)$ & $48(1)$ \\
\hline$C(9)$ & $3259(2)$ & $891(4)$ & $2735(2)$ & $48(1)$ \\
\hline$C(10)$ & $2944(2)$ & $2634(4)$ & $2876(2)$ & $39(1)$ \\
\hline$C(11)$ & $2190(2)$ & $2633(4)$ & $2166(2)$ & $40(1)$ \\
\hline$C(12)$ & $2845(2)$ & $-502(5)$ & $3033(3)$ & $65(1)$ \\
\hline$C(13)$ & $4093(2)$ & 715(5) & $3180(3)$ & $67(1)$ \\
\hline$C(14)$ & $2933(2)$ & $2921(4)$ & $3839(2)$ & $48(1)$ \\
\hline$C(15)$ & $3625(2)$ & $3980(5)$ & $4248(2)$ & $54(1)$ \\
\hline$C(16)$ & $3892(2)$ & $4626(4)$ & $3538(2)$ & $45(1)$ \\
\hline$C(17)$ & $3455(2)$ & $3993(4)$ & $2747(2)$ & $38(1)$ \\
\hline$C(18)$ & $3544(2)$ & $4621(4)$ & $1974(2)$ & $45(1)$ \\
\hline$C(19)$ & $4095(2)$ & $5788(4)$ & $2029(2)$ & $53(1)$ \\
\hline$C(20)$ & $4562(2)$ & $6310(5)$ & $2826(2)$ & $56(1)$ \\
\hline$C(21)$ & $4457(2)$ & $5750(5)$ & $3588(2)$ & $57(1)$ \\
\hline$C(22)$ & $2249(2)$ & $3765(5)$ & $3935(2)$ & $53(1)$ \\
\hline$C(23)$ & $2078(2)$ & $5363(6)$ & $3664(3)$ & $69(1)$ \\
\hline$C(24)$ & 1463(3) & $6146(9)$ & $3782(3)$ & $90(2)$ \\
\hline$C(25)$ & 1023(3) & $5344(12)$ & $4188(4)$ & $104(2)$ \\
\hline$C(26)$ & 1169(3) & $3757(12)$ & $4437(4)$ & $109(2)$ \\
\hline$C(27)$ & $1790(3)$ & $2942(7)$ & $4325(3)$ & $78(1)$ \\
\hline$N(1)$ & $2370(1)$ & $1790(3)$ & $1441(2)$ & $41(1)$ \\
\hline $\mathrm{O}(1)$ & 1198(2) & $2551(3)$ & $364(2)$ & $58(1)$ \\
\hline $\mathrm{O}(2)$ & $2129(2)$ & $821(3)$ & $-50(2)$ & $60(1)$ \\
\hline $\mathrm{O}(3)$ & $3878(2)$ & $4280(5)$ & $5020(2)$ & $81(1)$ \\
\hline$S(1)$ & $1737(1)$ & $1267(1)$ & $557(1)$ & $42(1)$ \\
\hline
\end{tabular}


Table 3. Bond lengths $[\AA]$ and angles $\left[{ }^{\circ}\right]$ for $3 a$.

\begin{tabular}{|c|c|}
\hline$C(1)-H(1 A)$ & 0.9600 \\
\hline$C(1)-H(1 B)$ & 0.9600 \\
\hline $\mathrm{C}(1)-\mathrm{H}(1 \mathrm{C})$ & 0.9600 \\
\hline$C(1)-C(2)$ & $1.511(5)$ \\
\hline$C(2)-C(3)$ & $1.386(5)$ \\
\hline$C(2)-C(7)$ & $1.380(5)$ \\
\hline $\mathrm{C}(3)-\mathrm{H}(3)$ & 0.9300 \\
\hline$C(3)-C(4)$ & $1.383(5)$ \\
\hline $\mathrm{C}(4)-\mathrm{H}(4)$ & 0.9300 \\
\hline$C(4)-C(5)$ & $1.385(5)$ \\
\hline$C(5)-C(6)$ & $1.387(4)$ \\
\hline$C(5)-S(1)$ & $1.757(3)$ \\
\hline $\mathrm{C}(6)-\mathrm{H}(6)$ & 0.9300 \\
\hline$C(6)-C(7)$ & $1.388(5)$ \\
\hline $\mathrm{C}(7)-\mathrm{H}(7)$ & 0.9300 \\
\hline $\mathrm{C}(8)-\mathrm{H}(8 \mathrm{~A})$ & 0.9700 \\
\hline $\mathrm{C}(8)-\mathrm{H}(8 \mathrm{~B})$ & 0.9700 \\
\hline $\mathrm{C}(8)-\mathrm{C}(9)$ & $1.530(5)$ \\
\hline $\mathrm{C}(8)-\mathrm{N}(1)$ & $1.481(4)$ \\
\hline$C(9)-C(10)$ & $1.581(4)$ \\
\hline$C(9)-C(12)$ & $1.531(5)$ \\
\hline$C(9)-C(13)$ & $1.528(5)$ \\
\hline$C(10)-C(11)$ & $1.537(4)$ \\
\hline$C(10)-C(14)$ & $1.568(4)$ \\
\hline$C(10)-C(17)$ & $1.521(4)$ \\
\hline $\mathrm{C}(11)-\mathrm{H}(11 \mathrm{~A})$ & 0.9700 \\
\hline $\mathrm{C}(11)-\mathrm{H}(11 \mathrm{~B})$ & 0.9700 \\
\hline $\mathrm{C}(11)-\mathrm{N}(1)$ & $1.474(4)$ \\
\hline $\mathrm{C}(12)-\mathrm{H}(12 \mathrm{~A})$ & 0.9600 \\
\hline $\mathrm{C}(12)-\mathrm{H}(12 \mathrm{~B})$ & 0.9600 \\
\hline $\mathrm{C}(12)-\mathrm{H}(12 \mathrm{C})$ & 0.9600 \\
\hline$C(13)-H(13 A)$ & 0.9600 \\
\hline $\mathrm{C}(13)-\mathrm{H}(13 \mathrm{~B})$ & 0.9600 \\
\hline $\mathrm{C}(13)-\mathrm{H}(13 \mathrm{C})$ & 0.9600 \\
\hline $\mathrm{C}(14)-\mathrm{H}(14)$ & 0.9800 \\
\hline$C(14)-C(15)$ & $1.539(5)$ \\
\hline
\end{tabular}




\begin{tabular}{|c|c|}
\hline$C(14)-C(22)$ & $1.507(5)$ \\
\hline$C(15)-C(16)$ & $1.471(5)$ \\
\hline$C(15)-O(3)$ & $1.214(4)$ \\
\hline$C(16)-C(17)$ & $1.393(4)$ \\
\hline$C(16)-C(21)$ & $1.388(5)$ \\
\hline$C(17)-C(18)$ & $1.396(4)$ \\
\hline $\mathrm{C}(18)-\mathrm{H}(18)$ & 0.9300 \\
\hline$C(18)-C(19)$ & $1.391(5)$ \\
\hline $\mathrm{C}(19)-\mathrm{H}(19)$ & 0.9300 \\
\hline$C(19)-C(20)$ & $1.388(5)$ \\
\hline $\mathrm{C}(20)-\mathrm{H}(20)$ & 0.9300 \\
\hline$C(20)-C(21)$ & $1.373(5)$ \\
\hline $\mathrm{C}(21)-\mathrm{H}(21)$ & 0.9300 \\
\hline$C(22)-C(23)$ & $1.382(6)$ \\
\hline$C(22)-C(27)$ & $1.380(5)$ \\
\hline $\mathrm{C}(23)-\mathrm{H}(23)$ & 0.9300 \\
\hline$C(23)-C(24)$ & $1.381(6)$ \\
\hline $\mathrm{C}(24)-\mathrm{H}(24)$ & 0.9300 \\
\hline$C(24)-C(25)$ & $1.361(9)$ \\
\hline $\mathrm{C}(25)-\mathrm{H}(25)$ & 0.9300 \\
\hline$C(25)-C(26)$ & $1.358(10)$ \\
\hline $\mathrm{C}(26)-\mathrm{H}(26)$ & 0.9300 \\
\hline$C(26)-C(27)$ & $1.400(9)$ \\
\hline $\mathrm{C}(27)-\mathrm{H}(27)$ & 0.9300 \\
\hline$N(1)-S(1)$ & $1.618(2)$ \\
\hline $\mathrm{O}(1)-\mathrm{S}(1)$ & $1.427(3)$ \\
\hline $\mathrm{O}(2)-\mathrm{S}(1)$ & $1.431(2)$ \\
\hline$H(1 A)-C(1)-H(1 B)$ & 109.5 \\
\hline$H(1 A)-C(1)-H(1 C)$ & 109.5 \\
\hline$H(1 B)-C(1)-H(1 C)$ & 109.5 \\
\hline$C(2)-C(1)-H(1 A)$ & 109.5 \\
\hline$C(2)-C(1)-H(1 B)$ & 109.5 \\
\hline $\mathrm{C}(2)-\mathrm{C}(1)-\mathrm{H}(1 \mathrm{C})$ & 109.5 \\
\hline$C(3)-C(2)-C(1)$ & $120.9(3)$ \\
\hline$C(7)-C(2)-C(1)$ & $121.2(3)$ \\
\hline$C(7)-C(2)-C(3)$ & $117.9(3)$ \\
\hline $\mathrm{C}(2)-\mathrm{C}(3)-\mathrm{H}(3)$ & 119.1 \\
\hline
\end{tabular}




\begin{tabular}{|c|c|}
\hline$C(4)-C(3)-C(2)$ & $121.9(3)$ \\
\hline $\mathrm{C}(4)-\mathrm{C}(3)-\mathrm{H}(3)$ & 119.1 \\
\hline $\mathrm{C}(3)-\mathrm{C}(4)-\mathrm{H}(4)$ & 120.3 \\
\hline$C(3)-C(4)-C(5)$ & 119.3(3) \\
\hline $\mathrm{C}(5)-\mathrm{C}(4)-\mathrm{H}(4)$ & 120.3 \\
\hline$C(4)-C(5)-C(6)$ & $119.8(3)$ \\
\hline$C(4)-C(5)-S(1)$ & $120.2(3)$ \\
\hline$C(6)-C(5)-S(1)$ & $120.0(2)$ \\
\hline $\mathrm{C}(5)-\mathrm{C}(6)-\mathrm{H}(6)$ & 120.2 \\
\hline$C(5)-C(6)-C(7)$ & $119.7(3)$ \\
\hline$C(7)-C(6)-H(6)$ & 120.2 \\
\hline$C(2)-C(7)-C(6)$ & $121.4(3)$ \\
\hline$C(2)-C(7)-H(7)$ & 119.3 \\
\hline $\mathrm{C}(6)-\mathrm{C}(7)-\mathrm{H}(7)$ & 119.3 \\
\hline $\mathrm{H}(8 \mathrm{~A})-\mathrm{C}(8)-\mathrm{H}(8 \mathrm{~B})$ & 109.0 \\
\hline $\mathrm{C}(9)-\mathrm{C}(8)-\mathrm{H}(8 \mathrm{~A})$ & 111.0 \\
\hline $\mathrm{C}(9)-\mathrm{C}(8)-\mathrm{H}(8 \mathrm{~B})$ & 111.0 \\
\hline $\mathrm{N}(1)-\mathrm{C}(8)-\mathrm{H}(8 \mathrm{~A})$ & 111.0 \\
\hline $\mathrm{N}(1)-\mathrm{C}(8)-\mathrm{H}(8 \mathrm{~B})$ & 111.0 \\
\hline$N(1)-C(8)-C(9)$ & $103.6(2)$ \\
\hline$C(8)-C(9)-C(10)$ & 101.3(2) \\
\hline$C(8)-C(9)-C(12)$ & $108.4(3)$ \\
\hline$C(12)-C(9)-C(10)$ & $112.1(3)$ \\
\hline$C(13)-C(9)-C(8)$ & 112.3(3) \\
\hline$C(13)-C(9)-C(10)$ & $113.2(3)$ \\
\hline$C(13)-C(9)-C(12)$ & 109.3(3) \\
\hline$C(11)-C(10)-C(9)$ & $101.3(2)$ \\
\hline$C(11)-C(10)-C(14)$ & $117.0(3)$ \\
\hline$C(14)-C(10)-C(9)$ & 112.9(3) \\
\hline$C(17)-C(10)-C(9)$ & 111.0(2) \\
\hline$C(17)-C(10)-C(11)$ & $112.4(2)$ \\
\hline$C(17)-C(10)-C(14)$ & 102.6(2) \\
\hline$C(10)-C(11)-H(11 A)$ & 111.4 \\
\hline$C(10)-C(11)-H(11 B)$ & 111.4 \\
\hline$H(11 A)-C(11)-H(11 B)$ & 109.2 \\
\hline$N(1)-C(11)-C(10)$ & 102.1(2) \\
\hline$N(1)-C(11)-H(11 A)$ & 111.4 \\
\hline $\mathrm{N}(1)-\mathrm{C}(11)-\mathrm{H}(11 \mathrm{~B})$ & 111.4 \\
\hline
\end{tabular}




\begin{tabular}{|c|c|}
\hline$C(9)-C(12)-H(12 A)$ & 109.5 \\
\hline $\mathrm{C}(9)-\mathrm{C}(12)-\mathrm{H}(12 \mathrm{~B})$ & 109.5 \\
\hline $\mathrm{C}(9)-\mathrm{C}(12)-\mathrm{H}(12 \mathrm{C})$ & 109.5 \\
\hline$H(12 A)-C(12)-H(12 B)$ & 109.5 \\
\hline$H(12 A)-C(12)-H(12 C)$ & 109.5 \\
\hline $\mathrm{H}(12 \mathrm{~B})-\mathrm{C}(12)-\mathrm{H}(12 \mathrm{C})$ & 109.5 \\
\hline$C(9)-C(13)-H(13 A)$ & 109.5 \\
\hline$C(9)-C(13)-H(13 B)$ & 109.5 \\
\hline$C(9)-C(13)-H(13 C)$ & 109.5 \\
\hline$H(13 A)-C(13)-H(13 B)$ & 109.5 \\
\hline$H(13 A)-C(13)-H(13 C)$ & 109.5 \\
\hline$H(13 B)-C(13)-H(13 C)$ & 109.5 \\
\hline $\mathrm{C}(10)-\mathrm{C}(14)-\mathrm{H}(14)$ & 109.0 \\
\hline$C(15)-C(14)-C(10)$ & $104.4(3)$ \\
\hline $\mathrm{C}(15)-\mathrm{C}(14)-\mathrm{H}(14)$ & 109.0 \\
\hline$C(22)-C(14)-C(10)$ & $115.4(3)$ \\
\hline$C(22)-C(14)-H(14)$ & 109.0 \\
\hline$C(22)-C(14)-C(15)$ & $109.9(3)$ \\
\hline$C(16)-C(15)-C(14)$ & $108.0(2)$ \\
\hline$O(3)-C(15)-C(14)$ & $125.3(3)$ \\
\hline$O(3)-C(15)-C(16)$ & $126.6(3)$ \\
\hline$C(17)-C(16)-C(15)$ & 109.2(3) \\
\hline$C(21)-C(16)-C(15)$ & $128.5(3)$ \\
\hline$C(21)-C(16)-C(17)$ & $122.2(3)$ \\
\hline$C(16)-C(17)-C(10)$ & $111.8(3)$ \\
\hline$C(16)-C(17)-C(18)$ & $118.8(3)$ \\
\hline$C(18)-C(17)-C(10)$ & $129.4(3)$ \\
\hline $\mathrm{C}(17)-\mathrm{C}(18)-\mathrm{H}(18)$ & 120.8 \\
\hline$C(19)-C(18)-C(17)$ & $118.4(3)$ \\
\hline $\mathrm{C}(19)-\mathrm{C}(18)-\mathrm{H}(18)$ & 120.8 \\
\hline $\mathrm{C}(18)-\mathrm{C}(19)-\mathrm{H}(19)$ & 119.1 \\
\hline$C(20)-C(19)-C(18)$ & $121.8(3)$ \\
\hline$C(20)-C(19)-H(19)$ & 119.1 \\
\hline$C(19)-C(20)-H(20)$ & 120.0 \\
\hline$C(21)-C(20)-C(19)$ & $120.0(3)$ \\
\hline $\mathrm{C}(21)-\mathrm{C}(20)-\mathrm{H}(20)$ & 120.0 \\
\hline$C(16)-C(21)-H(21)$ & 120.8 \\
\hline$C(20)-C(21)-C(16)$ & $118.5(3)$ \\
\hline
\end{tabular}




\begin{tabular}{|c|c|}
\hline$C(20)-C(21)-H(21)$ & 120.8 \\
\hline$C(23)-C(22)-C(14)$ & $121.7(3)$ \\
\hline$C(27)-C(22)-C(14)$ & $119.4(4)$ \\
\hline$C(27)-C(22)-C(23)$ & $118.8(4)$ \\
\hline $\mathrm{C}(22)-\mathrm{C}(23)-\mathrm{H}(23)$ & 119.3 \\
\hline$C(24)-C(23)-C(22)$ & $121.3(5)$ \\
\hline $\mathrm{C}(24)-\mathrm{C}(23)-\mathrm{H}(23)$ & 119.3 \\
\hline $\mathrm{C}(23)-\mathrm{C}(24)-\mathrm{H}(24)$ & 120.2 \\
\hline$C(25)-C(24)-C(23)$ & $119.6(7)$ \\
\hline $\mathrm{C}(25)-\mathrm{C}(24)-\mathrm{H}(24)$ & 120.2 \\
\hline $\mathrm{C}(24)-\mathrm{C}(25)-\mathrm{H}(25)$ & 120.0 \\
\hline$C(26)-C(25)-C(24)$ & $120.0(6)$ \\
\hline $\mathrm{C}(26)-\mathrm{C}(25)-\mathrm{H}(25)$ & 120.0 \\
\hline $\mathrm{C}(25)-\mathrm{C}(26)-\mathrm{H}(26)$ & 119.4 \\
\hline$C(25)-C(26)-C(27)$ & $121.2(6)$ \\
\hline $\mathrm{C}(27)-\mathrm{C}(26)-\mathrm{H}(26)$ & 119.4 \\
\hline$C(22)-C(27)-C(26)$ & $118.9(6)$ \\
\hline $\mathrm{C}(22)-\mathrm{C}(27)-\mathrm{H}(27)$ & 120.5 \\
\hline $\mathrm{C}(26)-\mathrm{C}(27)-\mathrm{H}(27)$ & 120.5 \\
\hline$C(8)-N(1)-S(1)$ & $119.9(2)$ \\
\hline$C(11)-N(1)-C(8)$ & $112.2(2)$ \\
\hline$C(11)-N(1)-S(1)$ & $122.3(2)$ \\
\hline$N(1)-S(1)-C(5)$ & $108.18(14)$ \\
\hline $\mathrm{O}(1)-\mathrm{S}(1)-\mathrm{C}(5)$ & $107.49(15)$ \\
\hline $\mathrm{O}(1)-\mathrm{S}(1)-\mathrm{N}(1)$ & $106.84(14)$ \\
\hline $\mathrm{O}(1)-\mathrm{S}(1)-\mathrm{O}(2)$ & $120.91(16)$ \\
\hline $\mathrm{O}(2)-\mathrm{S}(1)-\mathrm{C}(5)$ & $107.21(15)$ \\
\hline $\mathrm{O}(2)-\mathrm{S}(1)-\mathrm{N}(1)$ & $105.68(15)$ \\
\hline
\end{tabular}

Symmetry transformations used to generate equivalent atoms: 
Table 4. Anisotropic displacement parameters $\left(\AA^{2} \times 10^{3}\right)$ for 3a. The anisotropic displacement factor exponent takes the form: $-2 p^{2}\left[h^{2} a^{* 2} u^{11}+\ldots+2 h k a^{*} b^{*} u^{12}\right]$

\begin{tabular}{|c|c|c|c|c|c|c|}
\hline & $u^{11}$ & $u^{22}$ & $u^{33}$ & $u^{23}$ & $u^{13}$ & $u^{12}$ \\
\hline$C(1)$ & $78(3)$ & $51(2)$ & $104(3)$ & $2(2)$ & $44(3)$ & $-10(2)$ \\
\hline$C(2)$ & $51(2)$ & $45(2)$ & $62(2)$ & $-2(2)$ & $19(2)$ & $-3(2)$ \\
\hline$C(3)$ & $59(2)$ & $51(2)$ & $92(3)$ & $-11(2)$ & $46(2)$ & $-1(2)$ \\
\hline$C(4)$ & $54(2)$ & $36(2)$ & $79(2)$ & $-12(2)$ & $27(2)$ & $5(2)$ \\
\hline$C(5)$ & $38(2)$ & $34(2)$ & $42(2)$ & $-10(1)$ & $10(1)$ & $-4(1)$ \\
\hline$C(6)$ & $46(2)$ & $37(2)$ & $52(2)$ & $-8(1)$ & $21(1)$ & $2(1)$ \\
\hline$C(7)$ & $55(2)$ & $32(2)$ & $59(2)$ & $-6(1)$ & $21(2)$ & $4(1)$ \\
\hline$C(8)$ & $45(2)$ & $40(2)$ & $58(2)$ & $-7(1)$ & $12(2)$ & $4(1)$ \\
\hline $\mathrm{C}(9)$ & $45(2)$ & $41(2)$ & $52(2)$ & $5(1)$ & $6(1)$ & $6(1)$ \\
\hline$C(10)$ & $42(2)$ & $39(2)$ & $33(1)$ & $1(1)$ & $6(1)$ & $-1(1)$ \\
\hline$C(11)$ & $41(2)$ & $40(2)$ & $34(1)$ & $-5(1)$ & $6(1)$ & $4(1)$ \\
\hline$C(12)$ & $71(2)$ & $43(2)$ & $76(2)$ & $14(2)$ & $13(2)$ & $1(2)$ \\
\hline$C(13)$ & $53(2)$ & $60(2)$ & $77(3)$ & $8(2)$ & $2(2)$ & $17(2)$ \\
\hline$C(14)$ & $56(2)$ & $51(2)$ & $34(2)$ & $5(1)$ & $9(1)$ & $-8(2)$ \\
\hline$C(15)$ & $52(2)$ & $68(2)$ & $34(2)$ & $0(2)$ & $2(1)$ & $-6(2)$ \\
\hline$C(16)$ & $45(2)$ & $49(2)$ & $38(2)$ & $0(1)$ & $5(1)$ & $-1(2)$ \\
\hline$C(17)$ & $40(2)$ & $36(2)$ & $34(1)$ & $0(1)$ & $7(1)$ & $2(1)$ \\
\hline$C(18)$ & $52(2)$ & $43(2)$ & $39(2)$ & $-1(1)$ & $11(1)$ & $-2(2)$ \\
\hline$C(19)$ & $64(2)$ & $47(2)$ & $53(2)$ & $6(2)$ & $24(2)$ & $-2(2)$ \\
\hline$C(20)$ & $52(2)$ & $52(2)$ & $64(2)$ & $-4(2)$ & $19(2)$ & $-9(2)$ \\
\hline$C(21)$ & $49(2)$ & $64(2)$ & $51(2)$ & $-9(2)$ & $4(2)$ & $-12(2)$ \\
\hline$C(22)$ & $54(2)$ & $72(3)$ & $31(2)$ & $-12(2)$ & $10(1)$ & $-16(2)$ \\
\hline$C(23)$ & $65(2)$ & $78(3)$ & $64(2)$ & $-11(2)$ & $22(2)$ & $4(2)$ \\
\hline$C(24)$ & $73(3)$ & $113(4)$ & $76(3)$ & $-27(3)$ & $11(2)$ & $23(3)$ \\
\hline$C(25)$ & $51(3)$ & $174(7)$ & $81(4)$ & $-61(4)$ & $12(2)$ & $-6(4)$ \\
\hline$C(26)$ & $72(4)$ & $185(8)$ & $83(4)$ & $-45(4)$ & $42(3)$ & $-60(5)$ \\
\hline$C(27)$ & $71(3)$ & $113(4)$ & $54(2)$ & $-12(2)$ & $24(2)$ & $-33(3)$ \\
\hline $\mathrm{N}(1)$ & $43(1)$ & $38(1)$ & $41(1)$ & $-9(1)$ & $10(1)$ & $1(1)$ \\
\hline $\mathrm{O}(1)$ & $66(2)$ & $38(1)$ & $56(1)$ & $-1(1)$ & $-3(1)$ & $0(1)$ \\
\hline $\mathrm{O}(2)$ & $83(2)$ & $59(2)$ & $44(1)$ & $-10(1)$ & $28(1)$ & $-19(1)$ \\
\hline $\mathrm{O}(3)$ & $79(2)$ & $118(3)$ & $36(1)$ & $-5(2)$ & $0(1)$ & $-28(2)$ \\
\hline$S(1)$ & $53(1)$ & $35(1)$ & $36(1)$ & $-5(1)$ & $8(1)$ & $-5(1)$ \\
\hline
\end{tabular}


Table 5. Hydrogen coordinates $\left(\times 10^{4}\right)$ and isotropic displacement parameters $\left(\AA^{2} \times 10^{3}\right)$ for $3 a$.

\begin{tabular}{|c|c|c|c|c|}
\hline & $x$ & $y$ & $z$ & $U(e q)$ \\
\hline$H(1 A)$ & 494 & -5268 & 1914 & 111 \\
\hline $\mathrm{H}(1 \mathrm{~B})$ & 233 & -5696 & 918 & 111 \\
\hline $\mathrm{H}(1 \mathrm{C})$ & -271 & -4613 & 1321 & 111 \\
\hline $\mathrm{H}(3)$ & -24 & -1708 & 1540 & 75 \\
\hline $\mathrm{H}(4)$ & 524 & 640 & 1216 & 65 \\
\hline $\mathrm{H}(6)$ & 1952 & -2135 & 401 & 53 \\
\hline $\mathrm{H}(7)$ & 1374 & -4467 & 700 & 57 \\
\hline $\mathrm{H}(8 \mathrm{~A})$ & 3458 & 1321 & 1543 & 58 \\
\hline $\mathrm{H}(8 \mathrm{~B})$ & 2990 & -292 & 1531 & 58 \\
\hline$H(11 A)$ & 1817 & 2037 & 2352 & 48 \\
\hline $\mathrm{H}(11 \mathrm{~B})$ & 2015 & 3741 & 2005 & 48 \\
\hline$H(12 A)$ & 2320 & -383 & 2762 & 98 \\
\hline $\mathrm{H}(12 \mathrm{~B})$ & 2948 & -462 & 3655 & 98 \\
\hline $\mathrm{H}(12 \mathrm{C})$ & 3007 & -1536 & 2868 & 98 \\
\hline$H(13 A)$ & 4253 & -357 & 3067 & 100 \\
\hline$H(13 B)$ & 4193 & 864 & 3797 & 100 \\
\hline$H(13 C)$ & 4357 & 1529 & 2956 & 100 \\
\hline $\mathrm{H}(14)$ & 2991 & 1864 & 4142 & 58 \\
\hline $\mathrm{H}(18)$ & 3241 & 4268 & 1435 & 54 \\
\hline $\mathrm{H}(19)$ & 4153 & 6230 & 1518 & 64 \\
\hline $\mathrm{H}(20)$ & 4947 & 7039 & 2844 & 67 \\
\hline $\mathrm{H}(21)$ & 4758 & 6116 & 4125 & 68 \\
\hline $\mathrm{H}(23)$ & 2382 & 5923 & 3397 & 82 \\
\hline $\mathrm{H}(24)$ & 1351 & 7215 & 3586 & 107 \\
\hline $\mathrm{H}(25)$ & 623 & 5882 & 4295 & 125 \\
\hline $\mathrm{H}(26)$ & 850 & 3202 & 4686 & 131 \\
\hline $\mathrm{H}(27)$ & 1891 & 1864 & 4511 & 94 \\
\hline
\end{tabular}


Table 6. Torsion angles $\left[^{\circ}\right]$ for $3 a$.

\begin{tabular}{|c|c|}
\hline$C(1)-C(2)-C(3)-C(4)$ & $-178.6(4)$ \\
\hline$C(1)-C(2)-C(7)-C(6)$ & $179.5(4)$ \\
\hline$C(2)-C(3)-C(4)-C(5)$ & $-1.0(6)$ \\
\hline$C(3)-C(2)-C(7)-C(6)$ & $0.2(6)$ \\
\hline$C(3)-C(4)-C(5)-C(6)$ & $0.4(5)$ \\
\hline$C(3)-C(4)-C(5)-S(1)$ & $-178.4(3)$ \\
\hline$C(4)-C(5)-C(6)-C(7)$ & $0.5(5)$ \\
\hline $\mathrm{C}(4)-\mathrm{C}(5)-S(1)-\mathrm{N}(1)$ & $86.6(3)$ \\
\hline$C(4)-C(5)-S(1)-O(1)$ & $-28.5(3)$ \\
\hline$C(4)-C(5)-S(1)-O(2)$ & $-159.9(3)$ \\
\hline$C(5)-C(6)-C(7)-C(2)$ & $-0.8(5)$ \\
\hline $\mathrm{C}(6)-\mathrm{C}(5)-\mathrm{S}(1)-\mathrm{N}(1)$ & $-92.2(3)$ \\
\hline $\mathrm{C}(6)-\mathrm{C}(5)-\mathrm{S}(1)-\mathrm{O}(1)$ & $152.7(2)$ \\
\hline $\mathrm{C}(6)-\mathrm{C}(5)-\mathrm{S}(1)-\mathrm{O}(2)$ & $21.3(3)$ \\
\hline$C(7)-C(2)-C(3)-C(4)$ & $0.7(6)$ \\
\hline$C(8)-C(9)-C(10)-C(11)$ & $43.7(3)$ \\
\hline$C(8)-C(9)-C(10)-C(14)$ & 169.6(3) \\
\hline$C(8)-C(9)-C(10)-C(17)$ & $-75.8(3)$ \\
\hline$C(8)-N(1)-S(1)-C(5)$ & $73.7(3)$ \\
\hline $\mathrm{C}(8)-\mathrm{N}(1)-\mathrm{S}(1)-\mathrm{O}(1)$ & $-170.8(2)$ \\
\hline $\mathrm{C}(8)-\mathrm{N}(1)-\mathrm{S}(1)-\mathrm{O}(2)$ & $-40.8(3)$ \\
\hline$C(9)-C(8)-N(1)-C(11)$ & $8.8(3)$ \\
\hline $\mathrm{C}(9)-\mathrm{C}(8)-\mathrm{N}(1)-\mathrm{S}(1)$ & $-145.3(2)$ \\
\hline $\mathrm{C}(9)-\mathrm{C}(10)-\mathrm{C}(11)-\mathrm{N}(1)$ & $-37.9(3)$ \\
\hline$C(9)-C(10)-C(14)-C(15)$ & 100.6(3) \\
\hline$C(9)-C(10)-C(14)-C(22)$ & $-138.7(3)$ \\
\hline$C(9)-C(10)-C(17)-C(16)$ & $-102.4(3)$ \\
\hline$C(9)-C(10)-C(17)-C(18)$ & $76.2(4)$ \\
\hline$C(10)-C(11)-N(1)-C(8)$ & 19.1(3) \\
\hline$C(10)-C(11)-N(1)-S(1)$ & $172.4(2)$ \\
\hline$C(10)-C(14)-C(15)-C(16)$ & $14.3(4)$ \\
\hline $\mathrm{C}(10)-\mathrm{C}(14)-\mathrm{C}(15)-\mathrm{O}(3)$ & $-168.9(4)$ \\
\hline$C(10)-C(14)-C(22)-C(23)$ & $-65.0(4)$ \\
\hline$C(10)-C(14)-C(22)-C(27)$ & 116.8(3) \\
\hline$C(10)-C(17)-C(18)-C(19)$ & $-174.9(3)$ \\
\hline$C(11)-C(10)-C(14)-C(15)$ & $-142.4(3)$ \\
\hline
\end{tabular}




\begin{tabular}{|c|c|}
\hline$C(11)-C(10)-C(14)-C(22)$ & $-21.7(4)$ \\
\hline$C(11)-C(10)-C(17)-C(16)$ & $145.0(3)$ \\
\hline$C(11)-C(10)-C(17)-C(18)$ & $-36.4(4)$ \\
\hline$C(11)-N(1)-S(1)-C(5)$ & $-77.6(3)$ \\
\hline $\mathrm{C}(11)-\mathrm{N}(1)-\mathrm{S}(1)-\mathrm{O}(1)$ & $37.8(3)$ \\
\hline $\mathrm{C}(11)-\mathrm{N}(1)-\mathrm{S}(1)-\mathrm{O}(2)$ & $167.8(2)$ \\
\hline$C(12)-C(9)-C(10)-C(11)$ & $-71.7(3)$ \\
\hline$C(12)-C(9)-C(10)-C(14)$ & $54.3(4)$ \\
\hline$C(12)-C(9)-C(10)-C(17)$ & $168.8(3)$ \\
\hline$C(13)-C(9)-C(10)-C(11)$ & $164.1(3)$ \\
\hline$C(13)-C(9)-C(10)-C(14)$ & $-70.0(4)$ \\
\hline$C(13)-C(9)-C(10)-C(17)$ & $44.6(4)$ \\
\hline$C(14)-C(10)-C(11)-N(1)$ & $-161.1(3)$ \\
\hline$C(14)-C(10)-C(17)-C(16)$ & $18.5(3)$ \\
\hline$C(14)-C(10)-C(17)-C(18)$ & $-162.9(3)$ \\
\hline$C(14)-C(15)-C(16)-C(17)$ & $-3.2(4)$ \\
\hline$C(14)-C(15)-C(16)-C(21)$ & $173.8(4)$ \\
\hline$C(14)-C(22)-C(23)-C(24)$ & $-177.8(4)$ \\
\hline$C(14)-C(22)-C(27)-C(26)$ & $178.0(4)$ \\
\hline$C(15)-C(14)-C(22)-C(23)$ & $52.7(4)$ \\
\hline$C(15)-C(14)-C(22)-C(27)$ & $-125.5(3)$ \\
\hline$C(15)-C(16)-C(17)-C(10)$ & $-10.1(4)$ \\
\hline$C(15)-C(16)-C(17)-C(18)$ & $171.2(3)$ \\
\hline$C(15)-C(16)-C(21)-C(20)$ & $-173.4(4)$ \\
\hline$C(16)-C(17)-C(18)-C(19)$ & $3.6(5)$ \\
\hline$C(17)-C(10)-C(11)-N(1)$ & $80.5(3)$ \\
\hline$C(17)-C(10)-C(14)-C(15)$ & $-18.9(3)$ \\
\hline$C(17)-C(10)-C(14)-C(22)$ & $101.8(3)$ \\
\hline$C(17)-C(16)-C(21)-C(20)$ & $3.2(6)$ \\
\hline$C(17)-C(18)-C(19)-C(20)$ & $1.2(5)$ \\
\hline$C(18)-C(19)-C(20)-C(21)$ & $-4.1(6)$ \\
\hline$C(19)-C(20)-C(21)-C(16)$ & $1.8(6)$ \\
\hline$C(21)-C(16)-C(17)-C(10)$ & $172.8(3)$ \\
\hline$C(21)-C(16)-C(17)-C(18)$ & $-6.0(5)$ \\
\hline$C(22)-C(14)-C(15)-C(16)$ & $-110.1(3)$ \\
\hline$C(22)-C(14)-C(15)-O(3)$ & $66.8(5)$ \\
\hline$C(22)-C(23)-C(24)-C(25)$ & $1.3(7)$ \\
\hline$C(23)-C(22)-C(27)-C(26)$ & $-0.3(6)$ \\
\hline
\end{tabular}




$\begin{array}{lc}C(23)-C(24)-C(25)-C(26) & -3.1(8) \\ C(24)-C(25)-C(26)-C(27) & 3.3(8) \\ C(25)-C(26)-C(27)-C(22) & -1.6(7) \\ C(27)-C(22)-C(23)-C(24) & 0.4(6) \\ N(1)-C(8)-C(9)-C(10) & -32.0(3) \\ N(1)-C(8)-C(9)-C(12) & 86.1(3) \\ N(1)-C(8)-C(9)-C(13) & -153.0(3) \\ O(3)-C(15)-C(16)-C(17) & -180.0(4) \\ O(3)-C(15)-C(16)-C(21) & -3.1(7) \\ S(1)-C(5)-C(6)-C(7) & 179.3(3)\end{array}$

Symmetry transformations used to generate equivalent atoms: 
Table 7. Hydrogen bonds for $3 a\left[\AA\right.$ and $\left.{ }^{\circ}\right]$.

\begin{tabular}{lcccc}
\hline $\mathrm{D}-\mathrm{H} \ldots \mathrm{A}$ & $\mathrm{d}(\mathrm{D}-\mathrm{H})$ & $\mathrm{d}(\mathrm{H} \ldots \mathrm{A})$ & $\mathrm{d}(\mathrm{D} \ldots \mathrm{A})$ & $<(\mathrm{DHA})$ \\
\hline $\mathrm{C}(18)-\mathrm{H}(18) \ldots \mathrm{O}(2) \# 1$ & 0.93 & 2.47 & $3.128(4)$ & 127.6 \\
\hline
\end{tabular}

Symmetry transformations used to generate equivalent atoms:

$\# 1-x+1 / 2, y+1 / 2,-z$ 
CCDC: 2022309 (3a)

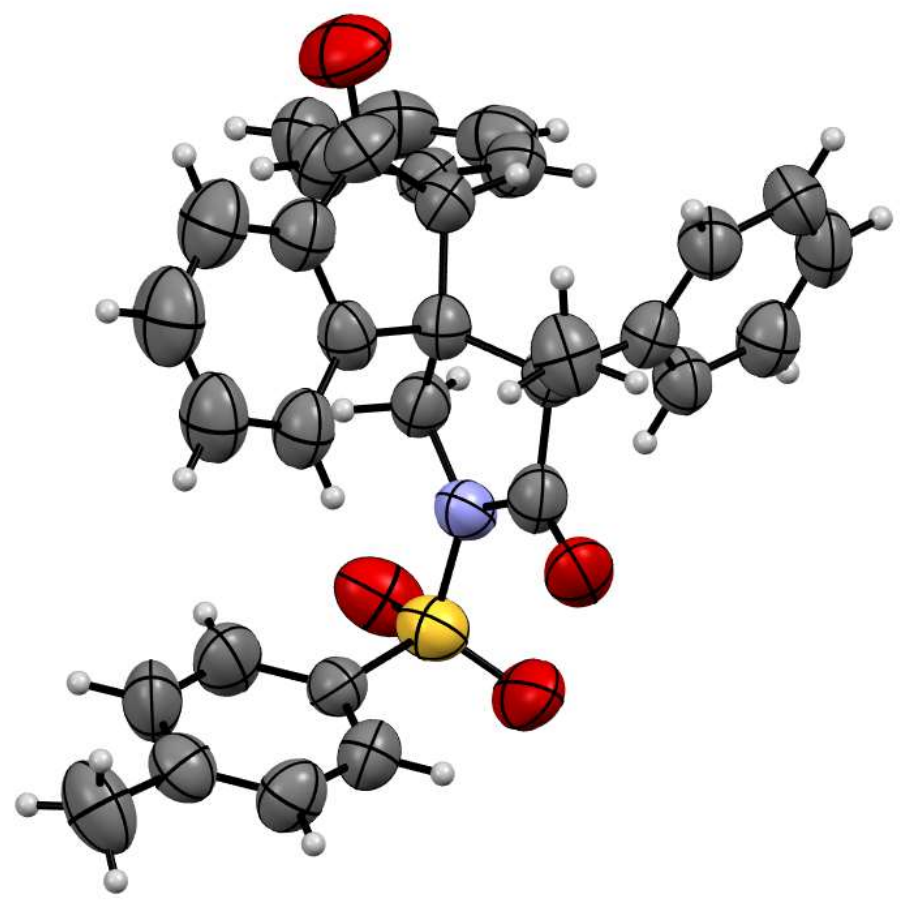


Table 8. Crystal data and structure refinement for $\mathbf{3 u}$.

\begin{tabular}{|c|c|}
\hline Empirical formula & $\mathrm{C}_{32} \mathrm{H}_{27} \mathrm{NO}_{4} \mathrm{~S}$ \\
\hline Formula weight & 521.60 \\
\hline Temperature/K & $296(2)$ \\
\hline Crystal system & monoclinic \\
\hline Space group & $\mathrm{P} 2{ }_{1} / \mathrm{n}$ \\
\hline $\mathrm{a} / \AA \AA$ & $9.002(5)$ \\
\hline $\mathrm{b} / \AA \AA$ & $20.705(11)$ \\
\hline$c / \AA ̊$ & $13.786(7)$ \\
\hline$\alpha /^{\circ}$ & 90 \\
\hline$\beta /^{\circ}$ & $92.886(6)$ \\
\hline $\mathrm{Y} /^{\circ}$ & 90 \\
\hline Volume $/ \AA^{3}$ & $2566(2)$ \\
\hline Z & 4 \\
\hline$\rho_{\text {calc }} \mathrm{g} / \mathrm{cm}^{3}$ & 1.350 \\
\hline$\mu / \mathrm{mm}^{-1}$ & 0.166 \\
\hline$F(000)$ & 1096.0 \\
\hline Crystal size $/ \mathrm{mm}^{3}$ & $0.120 \times 0.100 \times 0.060$ \\
\hline Radiation & $\operatorname{MoKa}(\lambda=0.71073)$ \\
\hline \multicolumn{2}{|c|}{$2 \Theta$ range for data collection $/^{\circ} 3.552$ to 49} \\
\hline Index ranges & $-10 \leq h \leq 10,-24 \leq \mathrm{k} \leq 24,-16 \leq \mathrm{I} \leq 16$ \\
\hline Reflections collected & 18746 \\
\hline Independent reflections & $4274\left[R_{\text {int }}=0.1210, R_{\text {sigma }}=0.1073\right]$ \\
\hline Data/restraints/parameters & $4274 / 0 / 345$ \\
\hline Goodness-of-fit on $F^{2}$ & 1.004 \\
\hline Final $R$ indexes $[\mid>=2 \sigma(I)]$ & $\mathrm{R}_{1}=0.0621, w \mathrm{R}_{2}=0.1279$ \\
\hline Final $R$ indexes [all data] & $\mathrm{R}_{1}=0.1760, \mathrm{wR}_{2}=0.1735$ \\
\hline Largest diff. peak/hole / e $\AA^{-}$ & $30.14 /-0.27$ \\
\hline
\end{tabular}


Table 9. Fractional Atomic Coordinates $\left(\times 10^{4}\right)$ and Equivalent Isotropic Displacement Parameters $\left(\AA^{2} \times 10^{3}\right)$ for $3 \mathbf{u}$. $U_{\text {eq }}$ is defined as $1 / 3$ of of the trace of the orthogonalised Uı tensor.

\begin{tabular}{|c|c|c|c|c|}
\hline Atom & $x$ & $y$ & $z$ & $\mathrm{U}(\mathrm{eq})$ \\
\hline$S(1)$ & $1875.3(16)$ & $3342.2(7)$ & $5242.0(10)$ & $76.9(4)$ \\
\hline $\mathrm{O}(1)$ & $1143(4)$ & $3897.1(16)$ & $4850(2)$ & $99.0(12)$ \\
\hline $\mathrm{O}(2)$ & $2886(4)$ & $2986.5(16)$ & $4685(2)$ & $97.3(12)$ \\
\hline $\mathrm{O}(3)$ & $3007(5)$ & $5305(2)$ & $9725(3)$ & $116.2(14)$ \\
\hline $\mathrm{O}(4)$ & $4280(3)$ & $2727.9(16)$ & $6613(3)$ & $86.1(11)$ \\
\hline $\mathrm{N}(1)$ & $2807(4)$ & $3614.6(17)$ & $6230(3)$ & $65.2(10)$ \\
\hline$C(1)$ & $2743(9)$ & $6544(3)$ & $6425(4)$ & $96.2(18)$ \\
\hline$C(2)$ & $1669(7)$ & $6293(3)$ & $6978(5)$ & $98.7(18)$ \\
\hline$C(3)$ & 1954(6) & $5753(2)$ & $7548(4)$ & $80.9(15)$ \\
\hline$C(4)$ & $3333(5)$ & $5456(2)$ & $7559(3)$ & $63.9(13)$ \\
\hline$C(5)$ & $3656(5)$ & $4866(2)$ & $8177(3)$ & $65.3(13)$ \\
\hline$C(6)$ & $3228(5)$ & $4207(2)$ & 7697(3) & $60.9(12)$ \\
\hline $\mathrm{C}(7)$ & $2532(5)$ & $4257(2)$ & $6646(3)$ & $66.8(13)$ \\
\hline$C(8)$ & $558(5)$ & $2817(2)$ & $5683(3)$ & $61.6(12)$ \\
\hline $\mathrm{C}(9)$ & $865(5)$ & $2178(3)$ & $5798(3)$ & $72.2(14)$ \\
\hline$C(10)$ & $-183(6)$ & 1786(2) & $6185(4)$ & $79.6(15)$ \\
\hline$C(11)$ & $-1515(6)$ & $2015(3)$ & $6489(3)$ & $74.1(14)$ \\
\hline$C(12)$ & $-2618(6)$ & $1588(3)$ & $6973(4)$ & $107.8(19)$ \\
\hline$C(13)$ & 2092(5) & $3928(2)$ & $8363(4)$ & $66.9(13)$ \\
\hline$C(14)$ & $1265(5)$ & $3363(3)$ & $8254(4)$ & $80.4(15)$ \\
\hline$C(15)$ & $250(6)$ & $3210(3)$ & $8946(5)$ & $99.9(18)$ \\
\hline$C(16)$ & $45(7)$ & $3612(4)$ & $9719(5)$ & $113(2)$ \\
\hline$C(17)$ & $877(7)$ & $4159(3)$ & $9852(4)$ & $101.9(18)$ \\
\hline$C(18)$ & 1899(6) & $4316(3)$ & $9160(4)$ & $76.3(14)$ \\
\hline$C(19)$ & $2866(6)$ & $4886(3)$ & $9125(4)$ & $83.1(16)$ \\
\hline$C(20)$ & 4101(8) & $6262(3)$ & $6421(4)$ & $90.5(17)$ \\
\hline$C(21)$ & $4388(6)$ & $5717(3)$ & $6978(4)$ & $79.1(15)$ \\
\hline$C(22)$ & $4561(5)$ & $3724(2)$ & $7555(3)$ & 61.1(12) \\
\hline$C(23)$ & $5016(5)$ & $3338(2)$ & $8460(3)$ & $81.8(15)$ \\
\hline$C(24)$ & $5891(5)$ & $4085(2)$ & 7166(4) & $63.3(12)$ \\
\hline$C(25)$ & $5998(5)$ & $4234(2)$ & 6192(4) & $69.2(13)$ \\
\hline$C(26)$ & $7169(6)$ & $4598(2)$ & $5847(4)$ & $78.0(14)$ \\
\hline$C(27)$ & $8270(5)$ & $4818(2)$ & $6499(5)$ & $81.9(16)$ \\
\hline$C(28)$ & $8210(5)$ & $4673(2)$ & $7466(4)$ & $80.0(15)$ \\
\hline$C(29)$ & $7049(6)$ & $4304(2)$ & $7805(4)$ & $77.3(14)$ \\
\hline$C(30)$ & $3918(5)$ & $3275(3)$ & $6760(4)$ & $69.1(14)$ \\
\hline$C(31)$ & $-796(6)$ & $3064(3)$ & $5950(4)$ & $90.7(17)$ \\
\hline$C(32)$ & $-1809(6)$ & $2656(3)$ & $6347(4)$ & $94.2(17)$ \\
\hline
\end{tabular}


Table 10. Anisotropic Displacement Parameters $\left(\AA^{2} \times 10^{3}\right)$ for $3 u$. The Anisotropic displacement factor exponent takes the form: $-2 \pi^{2}\left[h^{2} a^{* 2} U_{11}+2 h k a * b * U_{12}+\ldots\right]$.

\begin{tabular}{|c|c|c|c|c|c|c|}
\hline Atom & $U_{11}$ & $\mathbf{U}_{22}$ & $\mathrm{U}_{33}$ & $\mathbf{U}_{23}$ & $\mathbf{U}_{13}$ & $U_{12}$ \\
\hline$S(1)$ & $82.0(10)$ & $76.0(10)$ & $72.5(9)$ & $-0.4(8)$ & $2.1(8)$ & $-11.1(8)$ \\
\hline $\mathrm{O}(1)$ & $112(3)$ & $82(3)$ & $99(3)$ & $23(2)$ & $-27(2)$ & $-12(2)$ \\
\hline $\mathrm{O}(2)$ & $100(3)$ & 108(3) & $88(3)$ & $-25(2)$ & $41(2)$ & $-21(2)$ \\
\hline $\mathrm{O}(3)$ & $150(4)$ & 118(3) & $83(3)$ & $-25(2)$ & $28(3)$ & $-12(3)$ \\
\hline $\mathrm{O}(4)$ & $76(2)$ & $59(2)$ & $123(3)$ & $-8(2)$ & $5(2)$ & $6.9(19)$ \\
\hline$N(1)$ & $59(2)$ & $57(3)$ & $79(3)$ & $-5(2)$ & $-2(2)$ & $-3(2)$ \\
\hline$C(1)$ & $119(5)$ & $72(4)$ & $96(5)$ & $-6(3)$ & $-4(4)$ & $-2(4)$ \\
\hline$C(2)$ & $76(4)$ & $91(5)$ & $129(5)$ & $-9(4)$ & $-1(4)$ & $11(4)$ \\
\hline$C(3)$ & $73(4)$ & $65(4)$ & $106(4)$ & $6(3)$ & $24(3)$ & $0(3)$ \\
\hline$C(4)$ & $67(3)$ & $58(3)$ & $67(3)$ & $-15(3)$ & $17(3)$ & $-2(3)$ \\
\hline$C(5)$ & $60(3)$ & $62(3)$ & $74(3)$ & $-9(3)$ & 11(3) & $0(2)$ \\
\hline$C(6)$ & $55(3)$ & $62(3)$ & $66(3)$ & $4(3)$ & $6(2)$ & $3(2)$ \\
\hline$C(7)$ & $61(3)$ & $61(3)$ & $80(4)$ & $-1(3)$ & $13(3)$ & $3(2)$ \\
\hline$C(8)$ & $62(3)$ & $60(3)$ & $64(3)$ & $-6(2)$ & $9(2)$ & $-3(3)$ \\
\hline$C(9)$ & $67(3)$ & $77(4)$ & $73(4)$ & $0(3)$ & $10(3)$ & $9(3)$ \\
\hline$C(10)$ & $87(4)$ & $65(3)$ & $87(4)$ & $6(3)$ & $11(3)$ & $13(3)$ \\
\hline$C(11)$ & $70(4)$ & $80(4)$ & $72(4)$ & $6(3)$ & $-3(3)$ & $-5(3)$ \\
\hline$C(12)$ & $80(4)$ & $125(5)$ & $118(5)$ & $31(4)$ & $5(3)$ & $-24(4)$ \\
\hline$C(13)$ & $55(3)$ & $59(3)$ & $87(4)$ & $10(3)$ & $8(3)$ & $-3(3)$ \\
\hline$C(14)$ & $71(3)$ & $83(4)$ & $90(4)$ & $11(3)$ & $23(3)$ & $2(3)$ \\
\hline$C(15)$ & $81(4)$ & $98(5)$ & $122(5)$ & $21(4)$ & $27(4)$ & $-4(3)$ \\
\hline$C(16)$ & $96(5)$ & $128(6)$ & $119(6)$ & $29(5)$ & $44(4)$ & $-7(4)$ \\
\hline$C(17)$ & $97(5)$ & $117(5)$ & $95(5)$ & $11(4)$ & $35(4)$ & $5(4)$ \\
\hline$C(18)$ & $73(4)$ & $75(4)$ & $82(4)$ & $5(3)$ & $15(3)$ & $4(3)$ \\
\hline$C(19)$ & $93(4)$ & $85(4)$ & $72(4)$ & $-8(3)$ & $11(3)$ & $5(3)$ \\
\hline$C(20)$ & $115(5)$ & $80(4)$ & $79(4)$ & $-9(3)$ & $23(4)$ & $-24(4)$ \\
\hline$C(21)$ & $78(4)$ & $73(4)$ & $88(4)$ & $-13(3)$ & $27(3)$ & $-9(3)$ \\
\hline$C(22)$ & $58(3)$ & $63(3)$ & $62(3)$ & $-1(3)$ & $6(2)$ & 2(3) \\
\hline$C(23)$ & $73(3)$ & $85(4)$ & $87(4)$ & $27(3)$ & $1(3)$ & $1(3)$ \\
\hline$C(24)$ & $60(3)$ & $66(3)$ & $65(3)$ & $3(3)$ & $14(3)$ & $7(3)$ \\
\hline$C(25)$ & $59(3)$ & $70(3)$ & $79(4)$ & $0(3)$ & $14(3)$ & $-5(3)$ \\
\hline$C(26)$ & $74(4)$ & $79(4)$ & $82(4)$ & $9(3)$ & $18(3)$ & $5(3)$ \\
\hline$C(27)$ & $59(4)$ & $71(4)$ & $119(5)$ & $7(3)$ & $29(4)$ & $0(3)$ \\
\hline$C(28)$ & $57(3)$ & $87(4)$ & $96(4)$ & $-9(3)$ & $3(3)$ & $-9(3)$ \\
\hline$C(29)$ & $65(3)$ & $86(4)$ & $81(4)$ & $0(3)$ & $8(3)$ & 2(3) \\
\hline$C(30)$ & $60(3)$ & $66(4)$ & $83(4)$ & $2(3)$ & $15(3)$ & 2(3) \\
\hline$C(31)$ & $75(4)$ & $64(4)$ & $133(5)$ & $-8(3)$ & $9(4)$ & $8(3)$ \\
\hline$C(32)$ & $58(3)$ & $96(5)$ & $130(5)$ & $-7(4)$ & $19(3)$ & $3(3)$ \\
\hline
\end{tabular}


Table 11. Bond Lengths for $3 u$.

$\begin{array}{llllll}\text { Atom } & \text { Atom } & \text { Length/A } & \text { Atom } & \text { Atom } & \text { Length/A } \\ \mathrm{S}(1) & \mathrm{O}(1) & 1.418(3) & \mathrm{C}(10) & \mathrm{C}(11) & 1.375(6) \\ \mathrm{S}(1) & \mathrm{O}(2) & 1.425(3) & \mathrm{C}(11) & \mathrm{C}(32) & 1.366(6) \\ \mathrm{S}(1) & \mathrm{N}(1) & 1.662(4) & \mathrm{C}(11) & \mathrm{C}(12) & 1.510(6) \\ \mathrm{S}(1) & \mathrm{C}(8) & 1.741(4) & \mathrm{C}(13) & \mathrm{C}(18) & 1.379(6) \\ \mathrm{O}(3) & \mathrm{C}(19) & 1.202(5) & \mathrm{C}(13) & \mathrm{C}(14) & 1.391(6) \\ \mathrm{O}(4) & \mathrm{C}(30) & 1.200(5) & \mathrm{C}(14) & \mathrm{C}(15) & 1.390(6) \\ \mathrm{N}(1) & \mathrm{C}(30) & 1.397(6) & \mathrm{C}(15) & \mathrm{C}(16) & 1.372(7) \\ \mathrm{N}(1) & \mathrm{C}(7) & 1.474(5) & \mathrm{C}(16) & \mathrm{C}(17) & 1.366(7) \\ \mathrm{C}(1) & \mathrm{C}(20) & 1.356(7) & \mathrm{C}(17) & \mathrm{C}(18) & 1.396(6) \\ \mathrm{C}(1) & \mathrm{C}(2) & 1.363(7) & \mathrm{C}(18) & \mathrm{C}(19) & 1.469(7) \\ \mathrm{C}(2) & \mathrm{C}(3) & 1.383(7) & \mathrm{C}(20) & \mathrm{C}(21) & 1.382(6) \\ \mathrm{C}(3) & \mathrm{C}(4) & 1.385(6) & \mathrm{C}(22) & \mathrm{C}(23) & 1.520(5) \\ \mathrm{C}(4) & \mathrm{C}(21) & 1.382(6) & \mathrm{C}(22) & \mathrm{C}(30) & 1.527(6) \\ \mathrm{C}(4) & \mathrm{C}(5) & 1.508(6) & \mathrm{C}(22) & \mathrm{C}(24) & 1.531(6) \\ \mathrm{C}(5) & \mathrm{C}(19) & 1.519(6) & \mathrm{C}(24) & \mathrm{C}(25) & 1.386(6) \\ \mathrm{C}(5) & \mathrm{C}(6) & 1.558(6) & \mathrm{C}(24) & \mathrm{C}(29) & 1.406(6) \\ \mathrm{C}(6) & \mathrm{C}(13) & 1.521(6) & \mathrm{C}(25) & \mathrm{C}(26) & 1.397(6) \\ \mathrm{C}(6) & \mathrm{C}(7) & 1.553(6) & \mathrm{C}(26) & \mathrm{C}(27) & 1.381(6) \\ \mathrm{C}(6) & \mathrm{C}(22) & 1.582(6) & \mathrm{C}(27) & \mathrm{C}(28) & 1.370(6) \\ \mathrm{C}(8) & \mathrm{C}(9) & 1.359(6) & \mathrm{C}(28) & \mathrm{C}(29) & 1.394(6) \\ \mathrm{C}(8) & \mathrm{C}(31) & 1.388(6) & \mathrm{C}(31) & \mathrm{C}(32) & 1.376(6) \\ \mathrm{C}(9) & \mathrm{C}(10) & 1.372(6) & & & \end{array}$


Table 12. Bond Angles for $3 u$.

\begin{tabular}{|c|c|c|c|c|c|c|c|}
\hline Atom & Atom & Atom & Angle $/^{\circ}$ & Atom & Atom & Atom & Angle $/^{\circ}$ \\
\hline $\mathrm{O}(1)$ & $S(1)$ & $\mathrm{O}(2)$ & $120.7(2)$ & $C(18)$ & $C(13)$ & $C(6)$ & $112.0(4)$ \\
\hline $\mathrm{O}(1)$ & $S(1)$ & $N(1)$ & 104.1(2) & $C(14)$ & $C(13)$ & $C(6)$ & $128.7(5)$ \\
\hline $\mathrm{O}(2)$ & $S(1)$ & $N(1)$ & $107.9(2)$ & $C(15)$ & $C(14)$ & $C(13)$ & $119.0(5)$ \\
\hline $\mathrm{O}(1)$ & $S(1)$ & $C(8)$ & 109.1(2) & $C(16)$ & $C(15)$ & $C(14)$ & $120.9(6)$ \\
\hline $\mathrm{O}(2)$ & $S(1)$ & $C(8)$ & $109.3(2)$ & $C(17)$ & $C(16)$ & $C(15)$ & $120.9(6)$ \\
\hline$N(1)$ & $S(1)$ & $C(8)$ & $104.5(2)$ & $C(16)$ & $C(17)$ & $C(18)$ & $118.5(6)$ \\
\hline$C(30)$ & $\mathrm{N}(1)$ & $C(7)$ & $112.3(4)$ & $C(13)$ & $C(18)$ & $C(17)$ & $121.5(5)$ \\
\hline$C(30)$ & $\mathrm{N}(1)$ & $S(1)$ & $125.2(3)$ & $C(13)$ & $C(18)$ & $C(19)$ & $110.1(5)$ \\
\hline $\mathrm{C}(7)$ & $\mathrm{N}(1)$ & $S(1)$ & $122.5(3)$ & $C(17)$ & $C(18)$ & $C(19)$ & $128.4(6)$ \\
\hline$C(20)$ & $C(1)$ & $C(2)$ & $120.2(6)$ & $\mathrm{O}(3)$ & $C(19)$ & $C(18)$ & $126.9(5)$ \\
\hline$C(1)$ & $C(2)$ & $C(3)$ & $120.6(5)$ & $\mathrm{O}(3)$ & $C(19)$ & $C(5)$ & $125.0(5)$ \\
\hline$C(2)$ & $C(3)$ & $C(4)$ & $120.4(5)$ & $C(18)$ & $C(19)$ & $C(5)$ & $108.1(5)$ \\
\hline$C(21)$ & $\mathrm{C}(4)$ & $C(3)$ & $117.5(5)$ & $C(1)$ & $C(20)$ & $C(21)$ & $119.5(5)$ \\
\hline$C(21)$ & $C(4)$ & $C(5)$ & 121.6(5) & $C(20)$ & $C(21)$ & $C(4)$ & $121.8(5)$ \\
\hline$C(3)$ & $C(4)$ & $C(5)$ & $120.9(4)$ & $C(23)$ & $\mathrm{C}(22)$ & $C(30)$ & $110.3(4)$ \\
\hline$C(4)$ & $C(5)$ & $C(19)$ & $112.3(4)$ & $C(23)$ & $C(22)$ & $C(24)$ & $111.2(4)$ \\
\hline$C(4)$ & $C(5)$ & $C(6)$ & $115.6(4)$ & $C(30)$ & $C(22)$ & $C(24)$ & $108.7(4)$ \\
\hline$C(19)$ & $C(5)$ & $C(6)$ & $105.8(4)$ & $C(23)$ & $C(22)$ & $C(6)$ & $113.9(4)$ \\
\hline$C(13)$ & $C(6)$ & $C(7)$ & $109.7(4)$ & $C(30)$ & $C(22)$ & $C(6)$ & $102.1(4)$ \\
\hline$C(13)$ & $C(6)$ & $C(5)$ & $103.6(4)$ & $C(24)$ & $\mathrm{C}(22)$ & $C(6)$ & $110.2(3)$ \\
\hline$C(7)$ & $C(6)$ & $C(5)$ & $114.7(4)$ & $C(25)$ & $C(24)$ & $C(29)$ & $116.7(4)$ \\
\hline$C(13)$ & $C(6)$ & $C(22)$ & 111.8(3) & $C(25)$ & $C(24)$ & $C(22)$ & $122.8(4)$ \\
\hline$C(7)$ & $C(6)$ & $C(22)$ & 101.5(3) & $C(29)$ & $C(24)$ & $C(22)$ & $120.5(4)$ \\
\hline$C(5)$ & $C(6)$ & $C(22)$ & $115.7(4)$ & $C(24)$ & $C(25)$ & $C(26)$ & $122.7(5)$ \\
\hline$N(1)$ & $C(7)$ & $C(6)$ & 103.6(3) & $C(27)$ & $C(26)$ & $C(25)$ & $119.0(5)$ \\
\hline$C(9)$ & $C(8)$ & $C(31)$ & $120.2(4)$ & $C(28)$ & $C(27)$ & $C(26)$ & $119.9(5)$ \\
\hline$C(9)$ & $C(8)$ & $S(1)$ & $120.7(4)$ & $C(27)$ & $C(28)$ & $C(29)$ & $120.9(5)$ \\
\hline$C(31)$ & $C(8)$ & $S(1)$ & $119.0(4)$ & $C(28)$ & $C(29)$ & $C(24)$ & $120.8(5)$ \\
\hline$C(8)$ & $C(9)$ & $C(10)$ & $118.7(5)$ & $\mathrm{O}(4)$ & $C(30)$ & $N(1)$ & $125.4(5)$ \\
\hline$C(9)$ & $C(10)$ & $C(11)$ & $122.8(5)$ & $\mathrm{O}(4)$ & $C(30)$ & $C(22)$ & $126.8(5)$ \\
\hline$C(32)$ & $C(11)$ & $C(10)$ & 117.2(5) & $\mathrm{N}(1)$ & $C(30)$ & $C(22)$ & $107.8(4)$ \\
\hline$C(32)$ & $C(11)$ & $C(12)$ & $120.4(5)$ & $C(32)$ & $C(31)$ & $C(8)$ & $119.2(5)$ \\
\hline$C(10)$ & $C(11)$ & $C(12)$ & $122.4(5)$ & $C(11)$ & $C(32)$ & $C(31)$ & $121.7(5)$ \\
\hline$C(18)$ & $C(13)$ & $C(14)$ & 119.2(5) & & & & \\
\hline
\end{tabular}


Table 13. Torsion Angles for $\mathbf{3 u}$.

\begin{tabular}{|c|c|c|c|c|c|c|c|c|c|}
\hline A & B & C & D & Angle $/^{\circ}$ & A & B & C & D & Angle $/^{\circ}$ \\
\hline $\mathrm{O}(1)$ & $S(1)$ & $N(1)$ & $C(30)$ & $-167.1(3)$ & $C(16)$ & $C(17)$ & $C(18)$ & $C(19)$ & $177.5(5)$ \\
\hline $\mathrm{O}(2)$ & $S(1)$ & $N(1)$ & $C(30)$ & $-37.8(4)$ & $C(13)$ & $C(18)$ & $C(19)$ & $\mathrm{O}(3)$ & $-177.0(5)$ \\
\hline$C(8)$ & $S(1)$ & $\mathrm{N}(1)$ & $C(30)$ & $78.4(4)$ & $C(17)$ & $C(18)$ & $C(19)$ & $\mathrm{O}(3)$ & $4.6(9)$ \\
\hline $\mathrm{O}(1)$ & $S(1)$ & $N(1)$ & $C(7)$ & $13.0(4)$ & $C(13)$ & $C(18)$ & $C(19)$ & $C(5)$ & $4.1(6)$ \\
\hline $\mathrm{O}(2)$ & $S(1)$ & $N(1)$ & $C(7)$ & $142.3(3)$ & $C(17)$ & $C(18)$ & $C(19)$ & $C(5)$ & $-174.3(5)$ \\
\hline$C(8)$ & $S(1)$ & $N(1)$ & $C(7)$ & $-101.5(4)$ & $C(4)$ & $C(5)$ & $C(19)$ & $\mathrm{O}(3)$ & $-58.0(7)$ \\
\hline$C(20)$ & $C(1)$ & $C(2)$ & $C(3)$ & $0.8(9)$ & $C(6)$ & $C(5)$ & $C(19)$ & $\mathrm{O}(3)$ & $175.0(5)$ \\
\hline$C(1)$ & $C(2)$ & $C(3)$ & $C(4)$ & $-0.7(8)$ & $C(4)$ & $C(5)$ & $C(19)$ & $C(18)$ & $121.0(4)$ \\
\hline$C(2)$ & $C(3)$ & $C(4)$ & $C(21)$ & $-0.3(7)$ & $C(6)$ & $C(5)$ & $C(19)$ & $C(18)$ & $-6.0(5)$ \\
\hline$C(2)$ & $C(3)$ & $C(4)$ & $C(5)$ & $-179.9(5)$ & $C(2)$ & $C(1)$ & $C(20)$ & $C(21)$ & $0.2(8)$ \\
\hline$C(21)$ & $C(4)$ & $C(5)$ & $C(19)$ & $146.1(5)$ & $C(1)$ & $C(20)$ & $C(21)$ & $C(4)$ & $-1.3(8)$ \\
\hline$C(3)$ & $C(4)$ & $C(5)$ & $C(19)$ & $-34.4(6)$ & $C(3)$ & $C(4)$ & $C(21)$ & $C(20)$ & $1.3(7)$ \\
\hline$C(21)$ & $C(4)$ & $C(5)$ & $C(6)$ & $-92.4(5)$ & $C(5)$ & $C(4)$ & $C(21)$ & $C(20)$ & $-179.1(4)$ \\
\hline$C(3)$ & $C(4)$ & $C(5)$ & $C(6)$ & $87.1(5)$ & $C(13)$ & $C(6)$ & $C(22)$ & $C(23)$ & $-36.3(5)$ \\
\hline$C(4)$ & $C(5)$ & $C(6)$ & $C(13)$ & $-119.3(4)$ & $C(7)$ & $C(6)$ & $C(22)$ & $C(23)$ & $-153.2(4)$ \\
\hline$C(19)$ & $C(5)$ & $C(6)$ & $C(13)$ & $5.6(4)$ & $C(5)$ & $C(6)$ & $C(22)$ & $C(23)$ & $82.0(5)$ \\
\hline$C(4)$ & $C(5)$ & $C(6)$ & $C(7)$ & $0.2(5)$ & $C(13)$ & $C(6)$ & $C(22)$ & $C(30)$ & $82.5(4)$ \\
\hline$C(19)$ & $C(5)$ & $C(6)$ & $C(7)$ & $125.1(4)$ & $C(7)$ & $C(6)$ & $C(22)$ & $C(30)$ & $-34.3(4)$ \\
\hline$C(4)$ & $C(5)$ & $C(6)$ & $C(22)$ & $117.9(4)$ & $C(5)$ & $C(6)$ & $C(22)$ & $C(30)$ & $-159.1(4)$ \\
\hline$C(19)$ & $C(5)$ & $C(6)$ & $C(22)$ & 7.2(4) & $C(13)$ & $C(6)$ & $C(22)$ & $C(24)$ & $-162.1(4)$ \\
\hline$C(30)$ & $N(1)$ & $C(7)$ & $C(6)$ & $-18.0(4)$ & $C(7)$ & $C(6)$ & $C(22)$ & $C(24)$ & $81.1(4)$ \\
\hline$S(1)$ & $N(1)$ & $C(7)$ & $C(6)$ & $161.9(3)$ & $C(5)$ & $C(6)$ & $C(22)$ & $C(24)$ & $-43.7(5)$ \\
\hline$C(13)$ & $\mathrm{C}(6)$ & $\mathrm{C}(7)$ & $\mathrm{N}(1)$ & $-86.5(4)$ & $C(23)$ & $C(22)$ & $C(24)$ & $C(25)$ & 151.3(4) \\
\hline$C(5)$ & $C(6)$ & $C(7)$ & $\mathrm{N}(1)$ & $157.4(3)$ & $C(30)$ & $C(22)$ & $C(24)$ & $C(25)$ & $29.8(6)$ \\
\hline$C(22)$ & $C(6)$ & $C(7)$ & $\mathrm{N}(1)$ & $31.9(4)$ & $C(6)$ & $C(22)$ & $C(24)$ & $C(25)$ & $-81.4(5)$ \\
\hline $\mathrm{O}(1)$ & $S(1)$ & $C(8)$ & $C(9)$ & $158.1(4)$ & $C(23)$ & $C(22)$ & $C(24)$ & $C(29)$ & $-31.1(6)$ \\
\hline $\mathrm{O}(2)$ & $S(1)$ & $\mathrm{C}(8)$ & $C(9)$ & $24.1(5)$ & $C(30)$ & $C(22)$ & $C(24)$ & $C(29)$ & $-152.7(4)$ \\
\hline $\mathrm{N}(1)$ & $S(1)$ & $C(8)$ & $C(9)$ & $-91.1(4)$ & $C(6)$ & $C(22)$ & $C(24)$ & $C(29)$ & $96.1(5)$ \\
\hline $\mathrm{O}(1)$ & $S(1)$ & $C(8)$ & $C(31)$ & $-24.2(5)$ & $C(29)$ & $C(24)$ & $C(25)$ & $C(26)$ & $-1.6(7)$ \\
\hline $\mathrm{O}(2)$ & $S(1)$ & $C(8)$ & $C(31)$ & $-158.1(4)$ & $C(22)$ & $C(24)$ & $C(25)$ & $C(26)$ & $176.0(4)$ \\
\hline $\mathrm{N}(1)$ & $S(1)$ & $C(8)$ & $C(31)$ & $86.6(4)$ & $C(24)$ & $C(25)$ & $C(26)$ & $C(27)$ & $0.2(7)$ \\
\hline$C(31)$ & $C(8)$ & $C(9)$ & $C(10)$ & $-0.4(7)$ & $C(25)$ & $C(26)$ & $C(27)$ & $C(28)$ & $0.6(7)$ \\
\hline$S(1)$ & $\mathrm{C}(8)$ & $C(9)$ & $C(10)$ & $177.4(4)$ & $C(26)$ & $C(27)$ & $C(28)$ & $C(29)$ & $0.1(7)$ \\
\hline$C(8)$ & $C(9)$ & $C(10)$ & $C(11)$ & $-1.9(7)$ & $C(27)$ & $C(28)$ & $C(29)$ & $C(24)$ & $-1.6(7)$ \\
\hline$C(9)$ & $C(10)$ & $C(11)$ & $C(32)$ & $3.3(8)$ & $C(25)$ & $C(24)$ & $C(29)$ & $C(28)$ & $2.3(7)$ \\
\hline$C(9)$ & $C(10)$ & $C(11)$ & $C(12)$ & $-176.0(5)$ & $C(22)$ & $C(24)$ & $C(29)$ & $C(28)$ & $-175.4(4)$ \\
\hline$C(7)$ & $C(6)$ & $C(13)$ & $C(18)$ & $-126.3(4)$ & $C(7)$ & $\mathrm{N}(1)$ & $C(30)$ & $\mathrm{O}(4)$ & $174.7(4)$ \\
\hline
\end{tabular}




\begin{tabular}{|c|c|c|c|c|c|c|c|c|c|}
\hline$C(5)$ & $C(6)$ & $C(13)$ & $C(18)$ & $-3.4(5)$ & $S(1)$ & $\mathrm{N}(1)$ & $C(30)$ & $\mathrm{O}(4)$ & $-5.2(7)$ \\
\hline$C(22)$ & $C(6)$ & $C(13)$ & $C(18)$ & $121.9(4)$ & $C(7)$ & $N(1)$ & $C(30)$ & $C(22)$ & $-4.7(5)$ \\
\hline$C(7)$ & $C(6)$ & $C(13)$ & $C(14)$ & $52.8(6)$ & $S(1)$ & $\mathrm{N}(1)$ & $C(30)$ & $C(22)$ & $175.3(3)$ \\
\hline$C(5)$ & $C(6)$ & $C(13)$ & $C(14)$ & $175.6(5)$ & $C(23)$ & $C(22)$ & $C(30)$ & $\mathrm{O}(4)$ & $-33.1(6)$ \\
\hline$C(22)$ & $C(6)$ & $C(13)$ & $C(14)$ & $-59.1(6)$ & $C(24)$ & $C(22)$ & $C(30)$ & $\mathrm{O}(4)$ & $89.0(5)$ \\
\hline$C(18)$ & $C(13)$ & $C(14)$ & $C(15)$ & $0.8(7)$ & $C(6)$ & $C(22)$ & $C(30)$ & $\mathrm{O}(4)$ & $-154.5(4)$ \\
\hline$C(6)$ & $C(13)$ & $C(14)$ & $C(15)$ & $-178.2(4)$ & $C(23)$ & $C(22)$ & $C(30)$ & $N(1)$ & $146.3(4)$ \\
\hline$C(13)$ & $C(14)$ & $C(15)$ & $C(16)$ & $0.9(8)$ & $C(24)$ & $C(22)$ & $C(30)$ & $N(1)$ & $-91.6(4)$ \\
\hline$C(14)$ & $C(15)$ & $C(16)$ & $C(17)$ & $-2.7(9)$ & $C(6)$ & $C(22)$ & $C(30)$ & $N(1)$ & $24.9(4)$ \\
\hline$C(15)$ & $C(16)$ & $C(17)$ & $C(18)$ & $2.6(9)$ & $\mathrm{C}(9)$ & $C(8)$ & $C(31)$ & $C(32)$ & $1.1(8)$ \\
\hline$C(14)$ & $C(13)$ & $C(18)$ & $\mathrm{C}(17)$ & $-0.9(7)$ & $S(1)$ & $C(8)$ & $C(31)$ & $C(32)$ & $-176.6(4)$ \\
\hline$C(6)$ & $C(13)$ & $C(18)$ & $C(17)$ & 178.3(4) & $C(10)$ & $C(11)$ & $C(32)$ & $C(31)$ & $-2.5(8)$ \\
\hline$C(14)$ & $C(13)$ & $C(18)$ & $C(19)$ & $-179.4(4)$ & $C(12)$ & $C(11)$ & $C(32)$ & $C(31)$ & $176.9(5)$ \\
\hline$C(6)$ & $C(13)$ & $C(18)$ & $C(19)$ & $-0.3(6)$ & $C(8)$ & $C(31)$ & $C(32)$ & $C(11)$ & $0.3(8)$ \\
\hline$C(16)$ & $\mathrm{C}(17)$ & $C(18)$ & $C(13)$ & $-0.8(8)$ & & & & & \\
\hline
\end{tabular}


Table 14. Hydrogen Atom Coordinates $\left(\AA \times 10^{4}\right)$ and Isotropic Displacement Parameters $\left(\AA^{2} \times 10^{3}\right)$ for $3 u$.

\begin{tabular}{|c|c|c|c|c|}
\hline Atom & $x$ & $y$ & $z$ & $\mathrm{U}(\mathrm{eq})$ \\
\hline$H(1)$ & 2542.65 & 6909.98 & 6050.29 & 115 \\
\hline$H(10)$ & 735.58 & 6485.84 & 6970.39 & 118 \\
\hline $\mathrm{H}(11)$ & 1215.99 & 5589.51 & 7927.53 & 97 \\
\hline$H(7)$ & 4728.61 & 4858.64 & 8339.81 & 78 \\
\hline $\mathrm{H}(5)$ & 1474.81 & 4346.79 & 6649.07 & 80 \\
\hline $\mathrm{H}(23)$ & 3011.33 & 4592.76 & 6284.34 & 80 \\
\hline $\mathrm{H}(4)$ & 1767.69 & 2009.63 & 5616.98 & 87 \\
\hline $\mathrm{H}(26)$ & 17.06 & 1347.19 & 6243.93 & 96 \\
\hline$H(3)$ & -2337.34 & 1548.66 & 7651.36 & 162 \\
\hline$H(2)$ & -2624.54 & 1168.06 & 6676.86 & 162 \\
\hline $\mathrm{H}(27)$ & -3592.62 & 1774.94 & 6897.66 & 162 \\
\hline $\mathrm{H}(14)$ & 1388.33 & 3091.98 & 7725.83 & 97 \\
\hline $\mathrm{H}(13)$ & -297.76 & 2829.76 & 8883.47 & 120 \\
\hline$H(6)$ & -668.72 & 3509.52 & 10159.26 & 136 \\
\hline $\mathrm{H}(12)$ & 764.92 & 4422.18 & 10390.33 & 122 \\
\hline$H(8)$ & 4835.03 & 6433.57 & 6046.11 & 109 \\
\hline$H(9)$ & 5315.42 & 5520.17 & 6962.12 & 95 \\
\hline $\mathrm{H}(16)$ & 5870.58 & 3078.68 & 8336.92 & 123 \\
\hline $\mathrm{H}(17)$ & 4209.37 & 3062.58 & 8627.23 & 123 \\
\hline $\mathrm{H}(15)$ & 5254.77 & 3628.33 & 8987.79 & 123 \\
\hline $\mathrm{H}(22)$ & 5261.05 & 4086.27 & 5749.88 & 83 \\
\hline $\mathrm{H}(21)$ & 7205.29 & 4690 & 5188.53 & 94 \\
\hline $\mathrm{H}(20)$ & 9050.97 & 5063.82 & 6281.32 & 98 \\
\hline $\mathrm{H}(19)$ & 8954.15 & 4823.01 & 7901.31 & 96 \\
\hline $\mathrm{H}(18)$ & 7040.93 & 4202.34 & 8461.44 & 93 \\
\hline $\mathrm{H}(24)$ & -1015.47 & 3499.44 & 5861.76 & 109 \\
\hline $\mathrm{H}(25)$ & -2717.95 & 2821.75 & 6522.13 & 113 \\
\hline
\end{tabular}




\section{Copies of the ${ }^{1} \mathrm{H},{ }^{19} \mathrm{~F}$ NMR and ${ }^{13} \mathrm{C}$ NMR spectra}

$3 a$
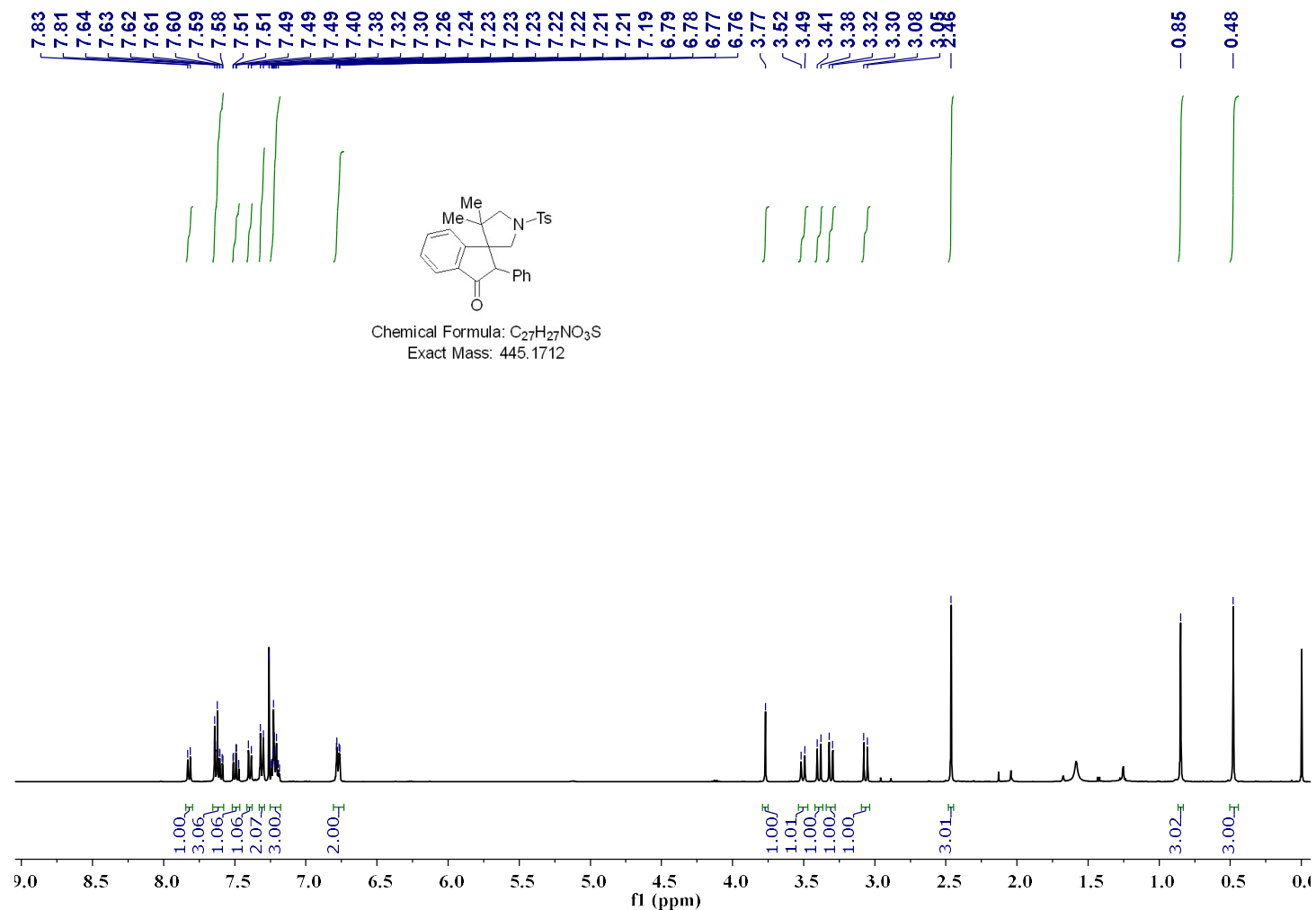

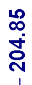

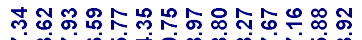

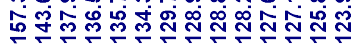

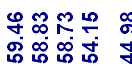

8กิ

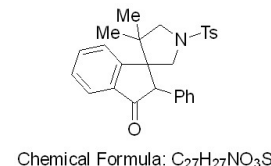

Chemical Formula: $\mathrm{C}_{27} \mathrm{H}_{27} \mathrm{NO}_{3} \mathrm{~S}$

Exact Mass: 445.1712

$\begin{array}{llllllllllll}220 & 210 & 200 & 190 & 180 & 170 & 160 & 150 & 140 & 130 & 120 & 11 \\ f 1 & (\mathrm{ppm})\end{array}$ 

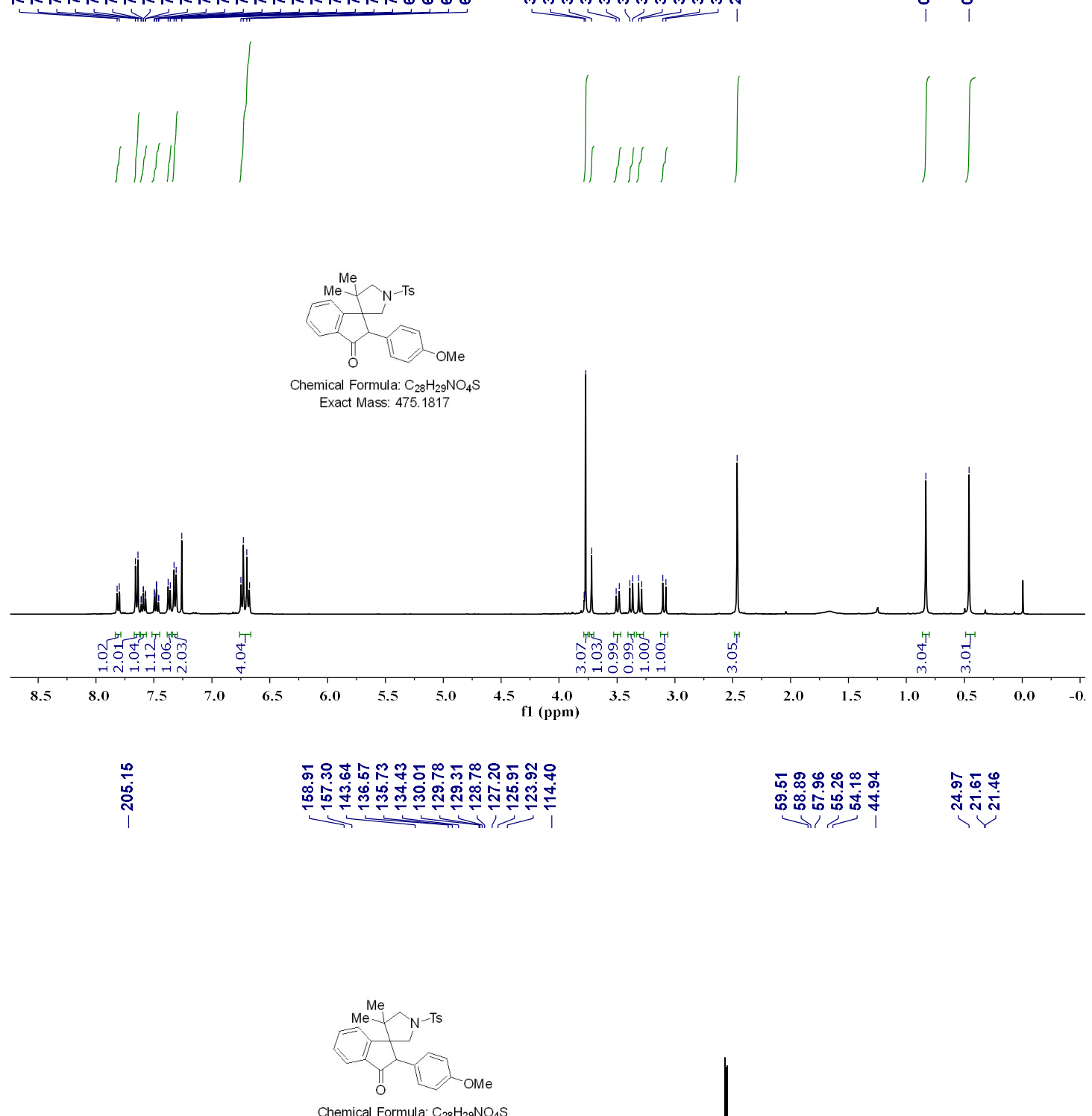

Exact Mass: 475.1817

\begin{tabular}{lllllllllllll}
\hline 30 & 220 & 210 & 200 & 190 & 180 & 170 & 160 & 150 & 140 & 130 & $\begin{array}{l}120 \\
\mathrm{f} 1(\mathrm{ppm})\end{array}$
\end{tabular} 
(1)

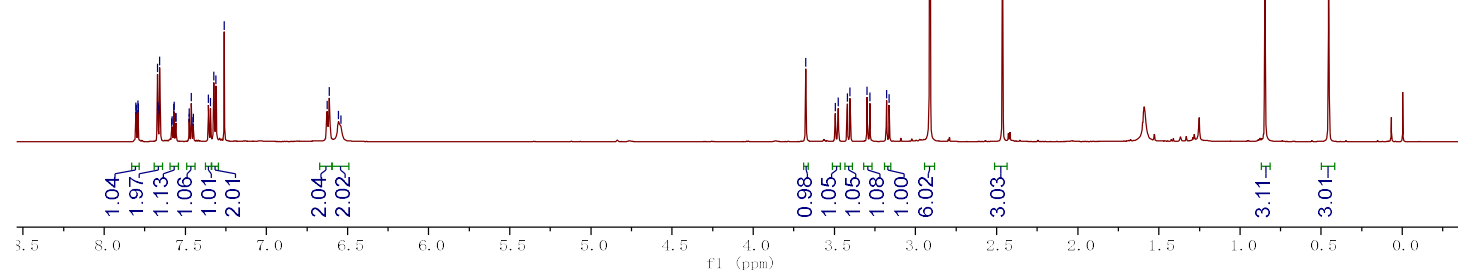

$$
{ }_{\mathrm{C}}^{\mathrm{Me}}
$$
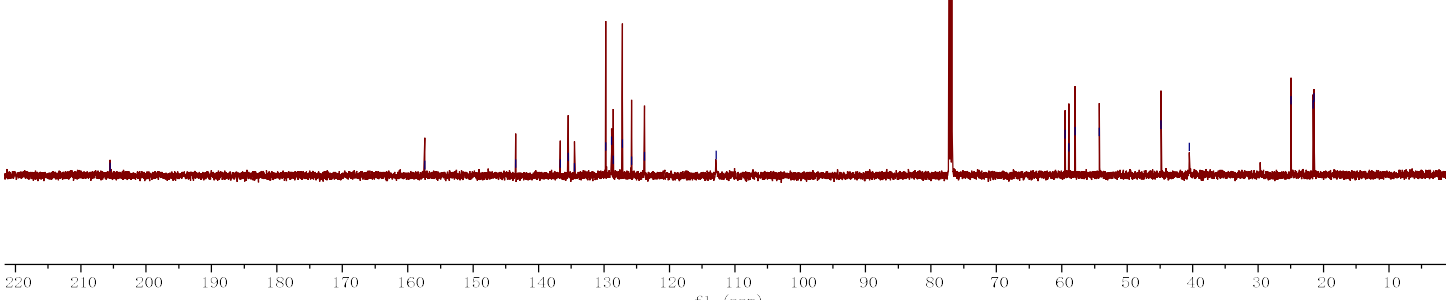


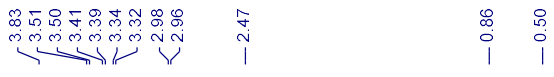
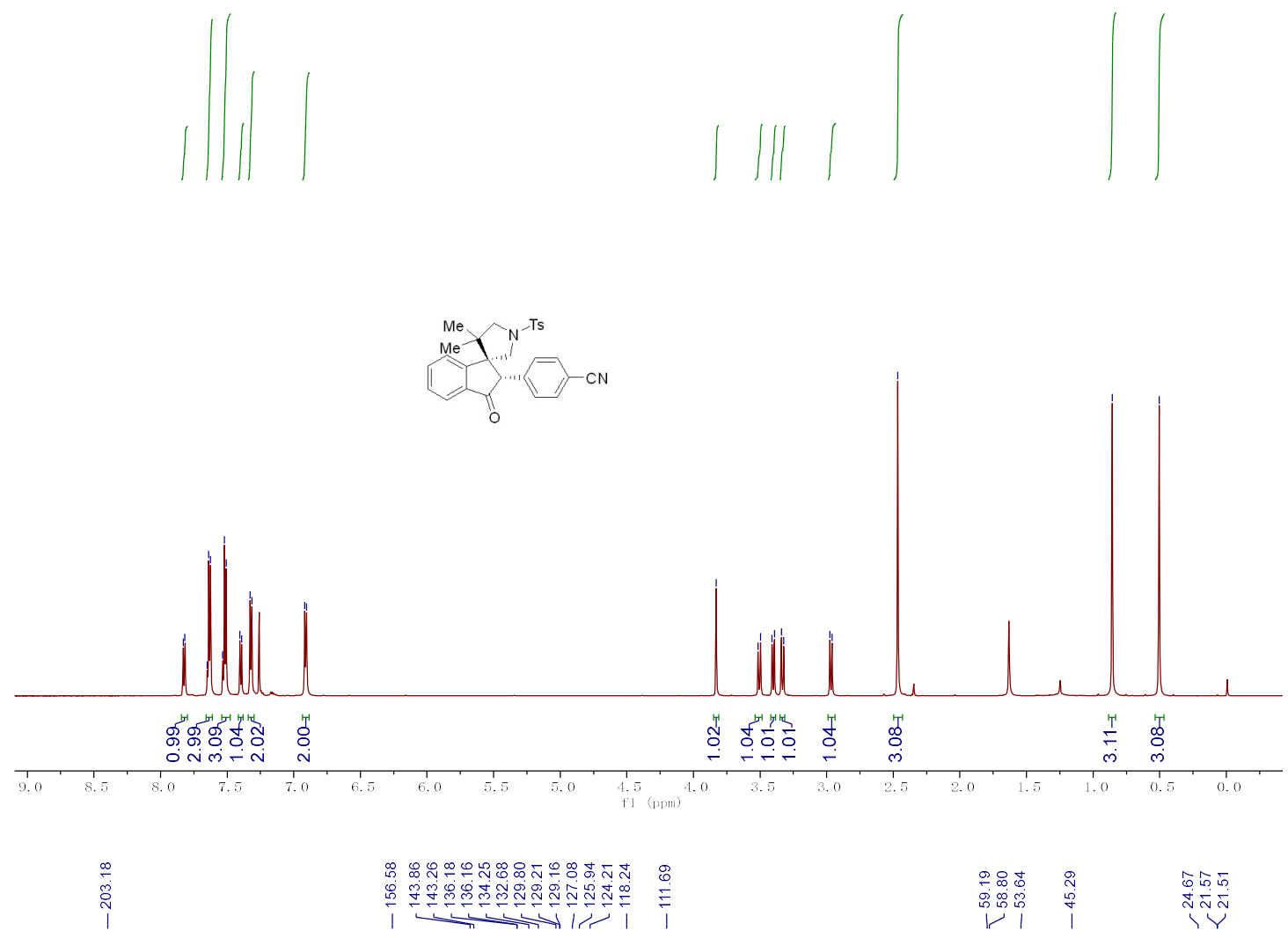

$$
\text { CN }
$$

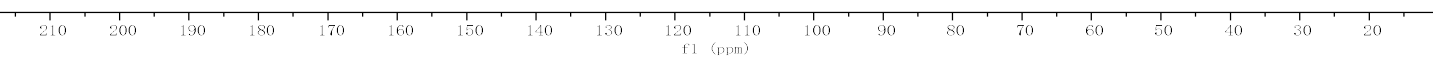


$3 \mathbf{e}$

̄

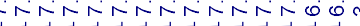

(1, N

IIIII
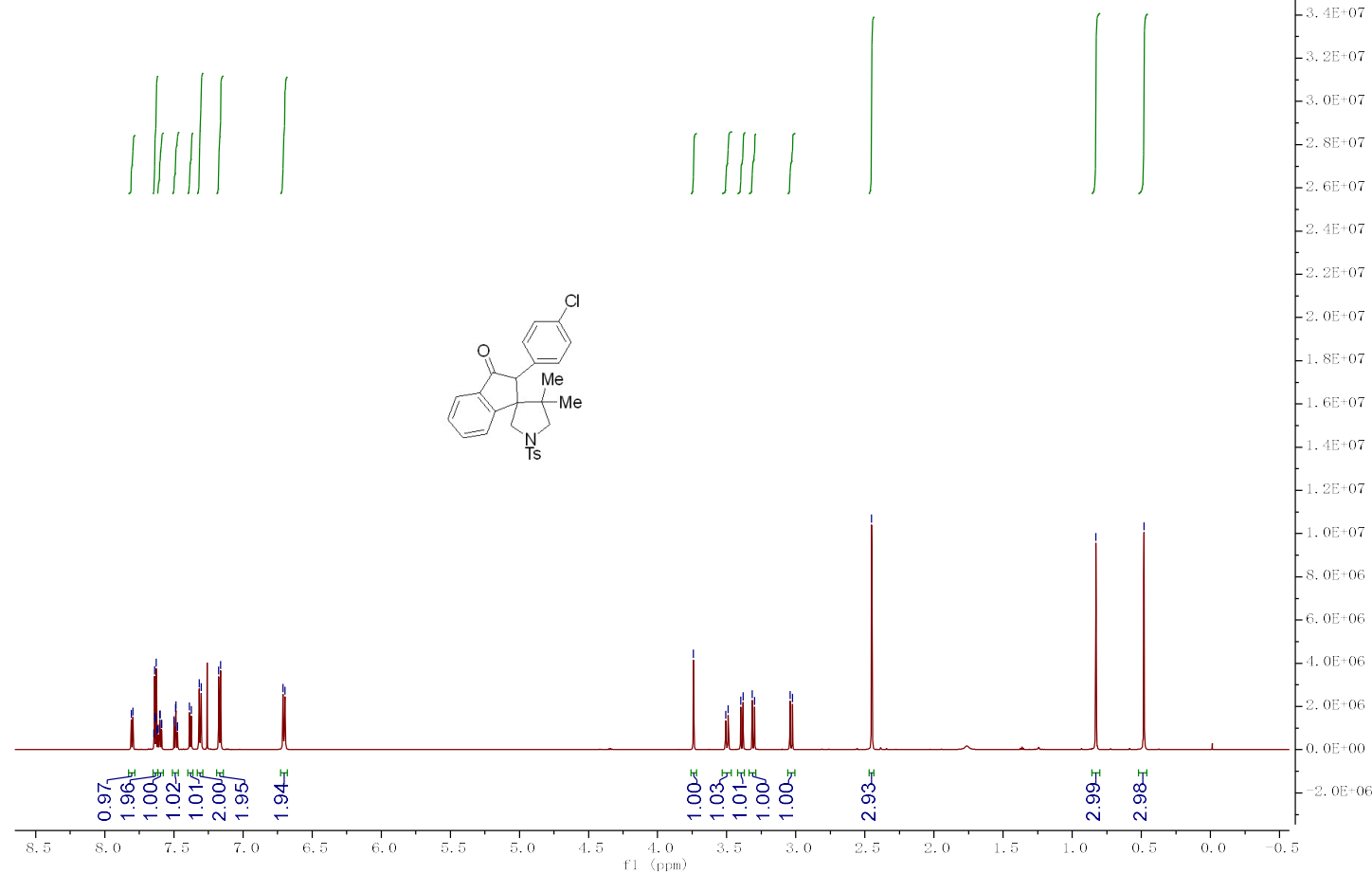

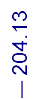

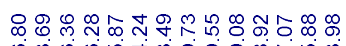

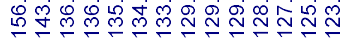

I

잉용우요요

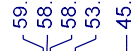

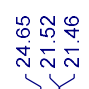

5000000

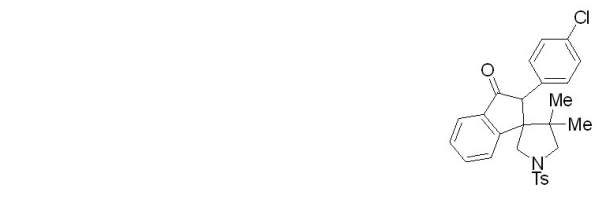

500000

000000
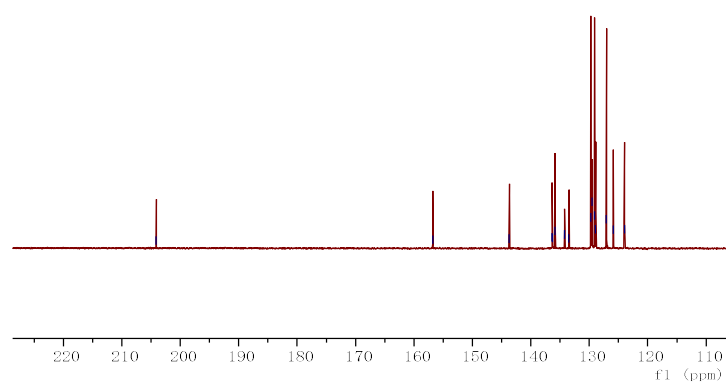

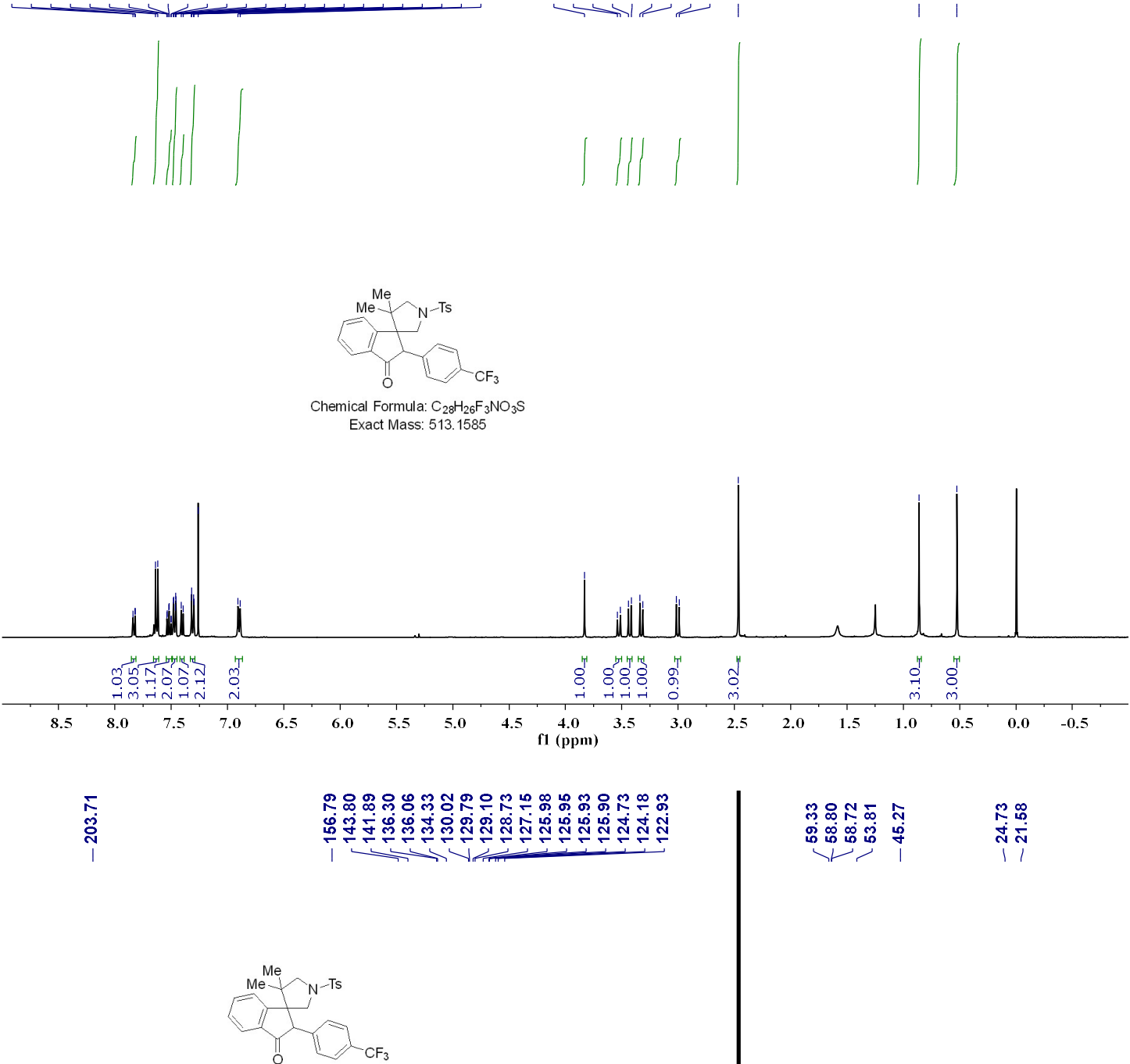
Exact Mass: 513.1585
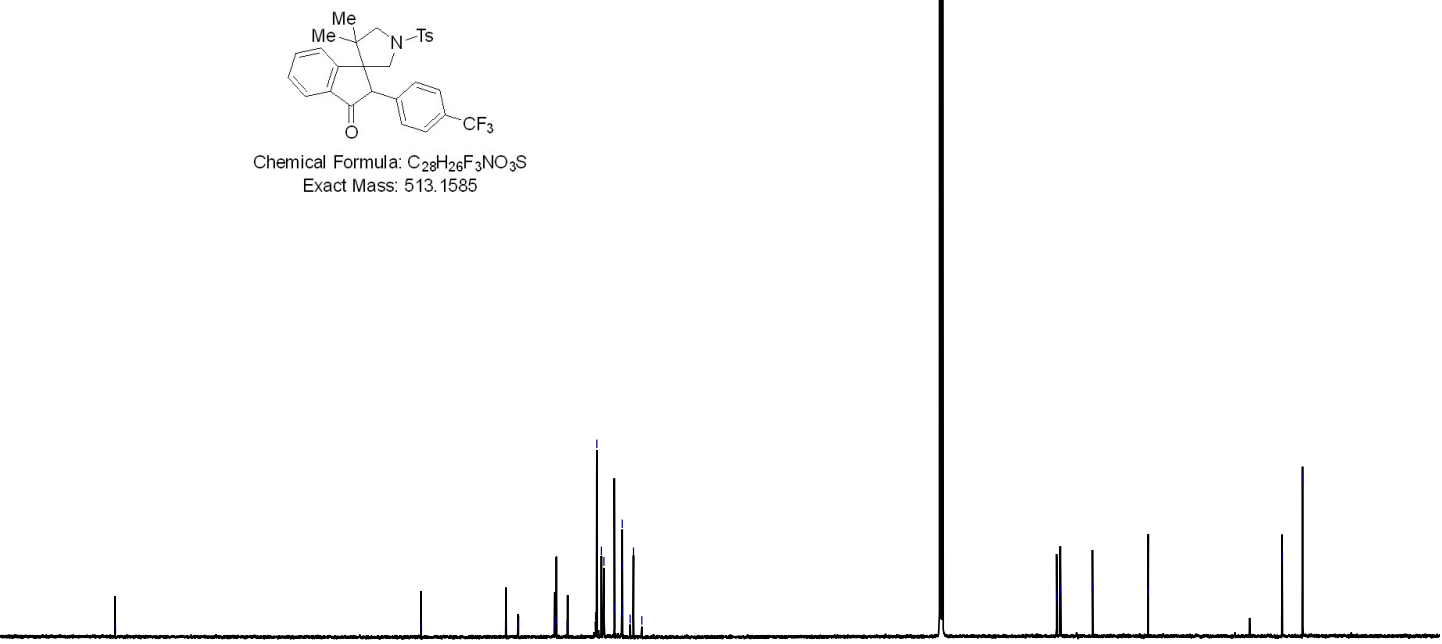

\begin{tabular}{llllllllllllllllllllll}
\hline 220 & 210 & 200 & 190 & 180 & 170 & 160 & 150 & 140 & 130 & 120 & 110 & 100 & 90 & 80 & 70 & 60 & 50 & 40 & 30 & 20 & 10
\end{tabular} 


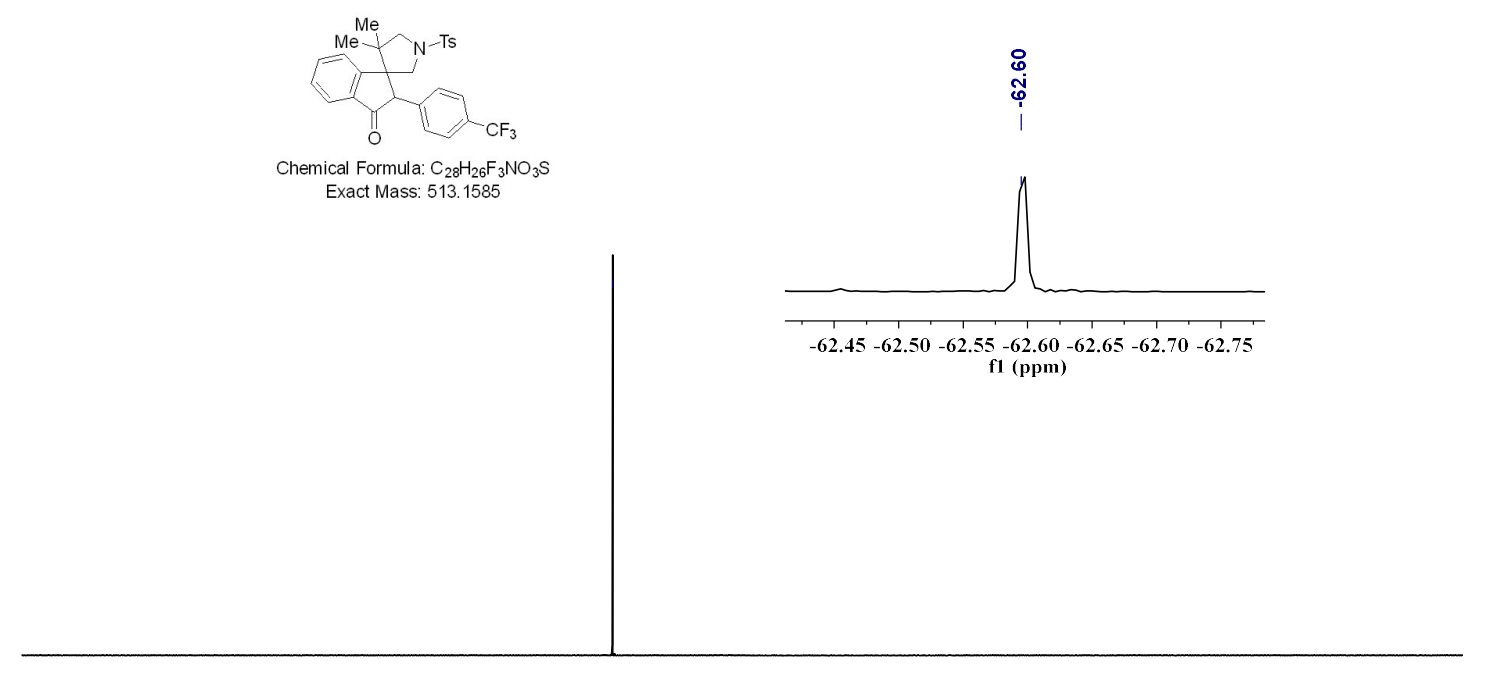

$\begin{array}{lllllllllllllllllllllllllll}100 & 80 & 60 & 40 & 20 & 0 & -20 & -40 & -60 & -80 & -100 & -120 & -140 & -160 & -180 & -200 & -220 & -240 & -260 & -280 & -300\end{array}$ 
$3 g$

ஜ

$\underbrace{\infty} \underbrace{\infty}$
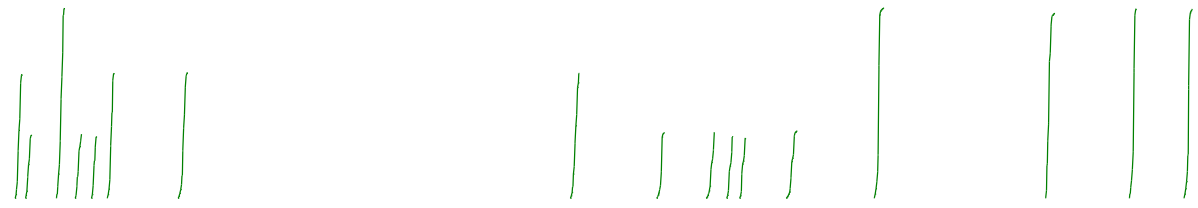

-COOEt

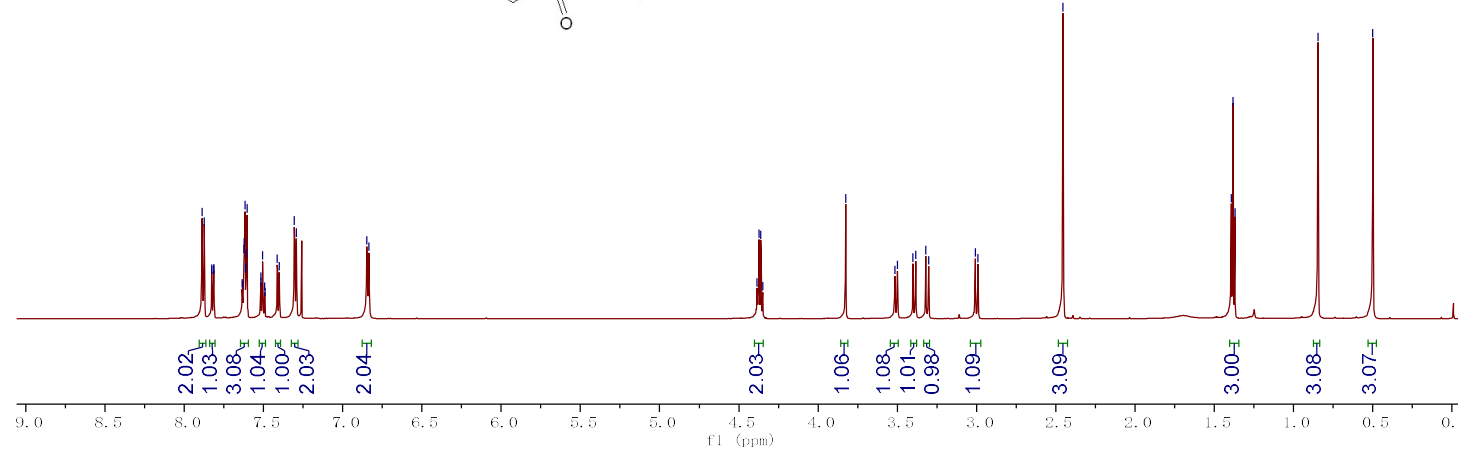

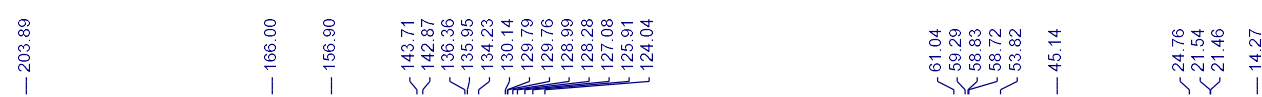

-COOEt
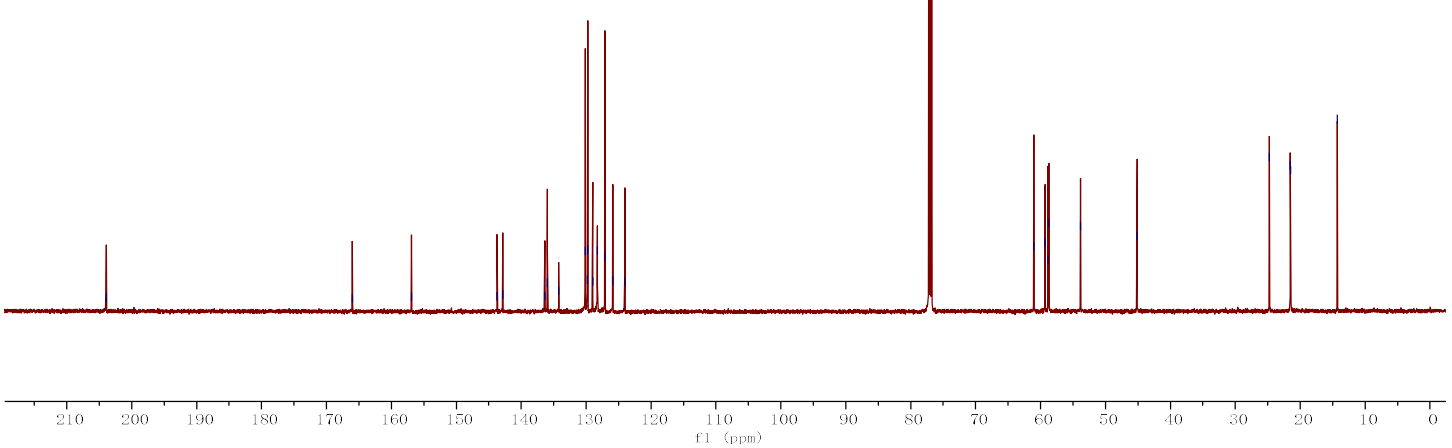

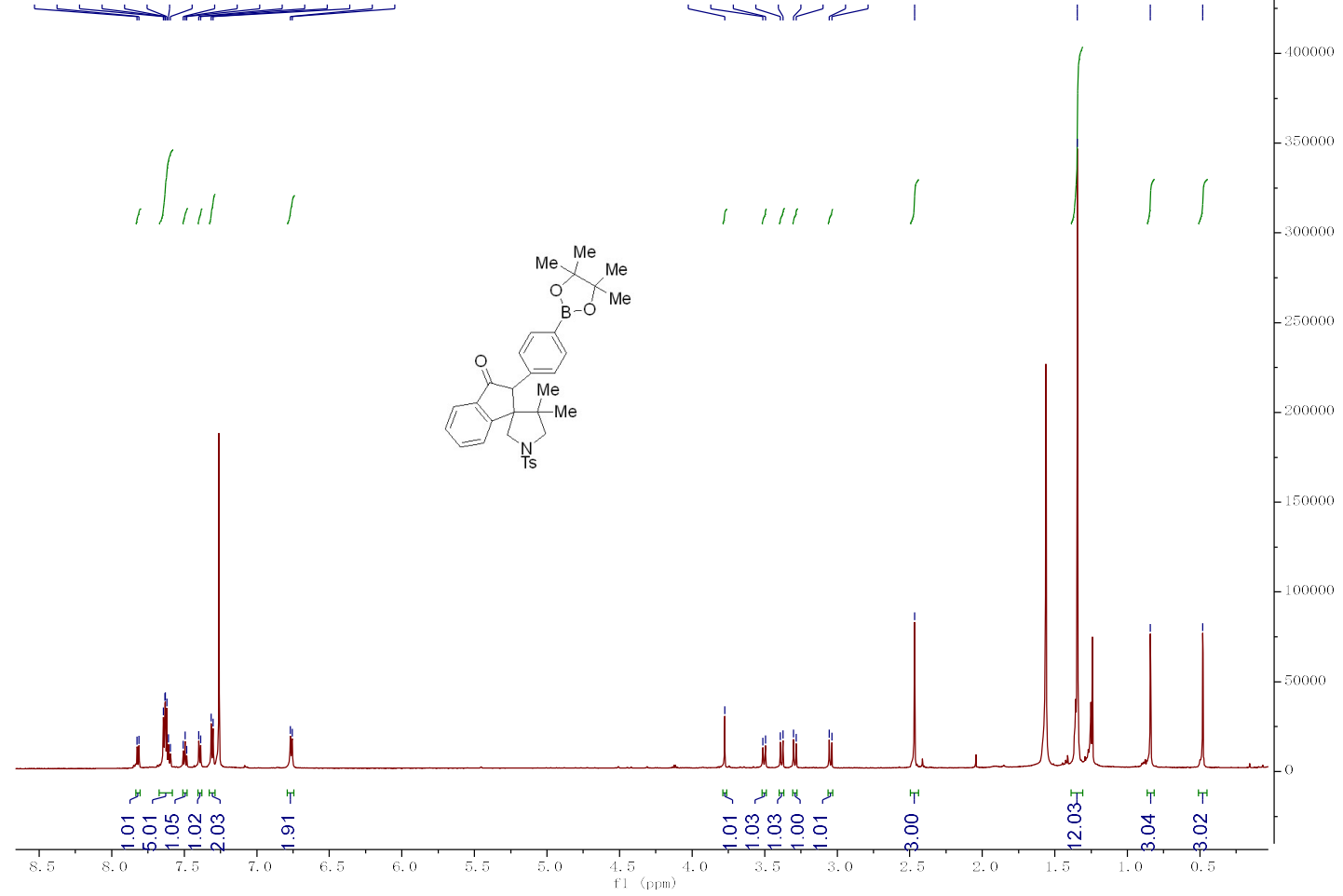
in

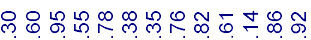

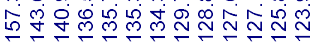
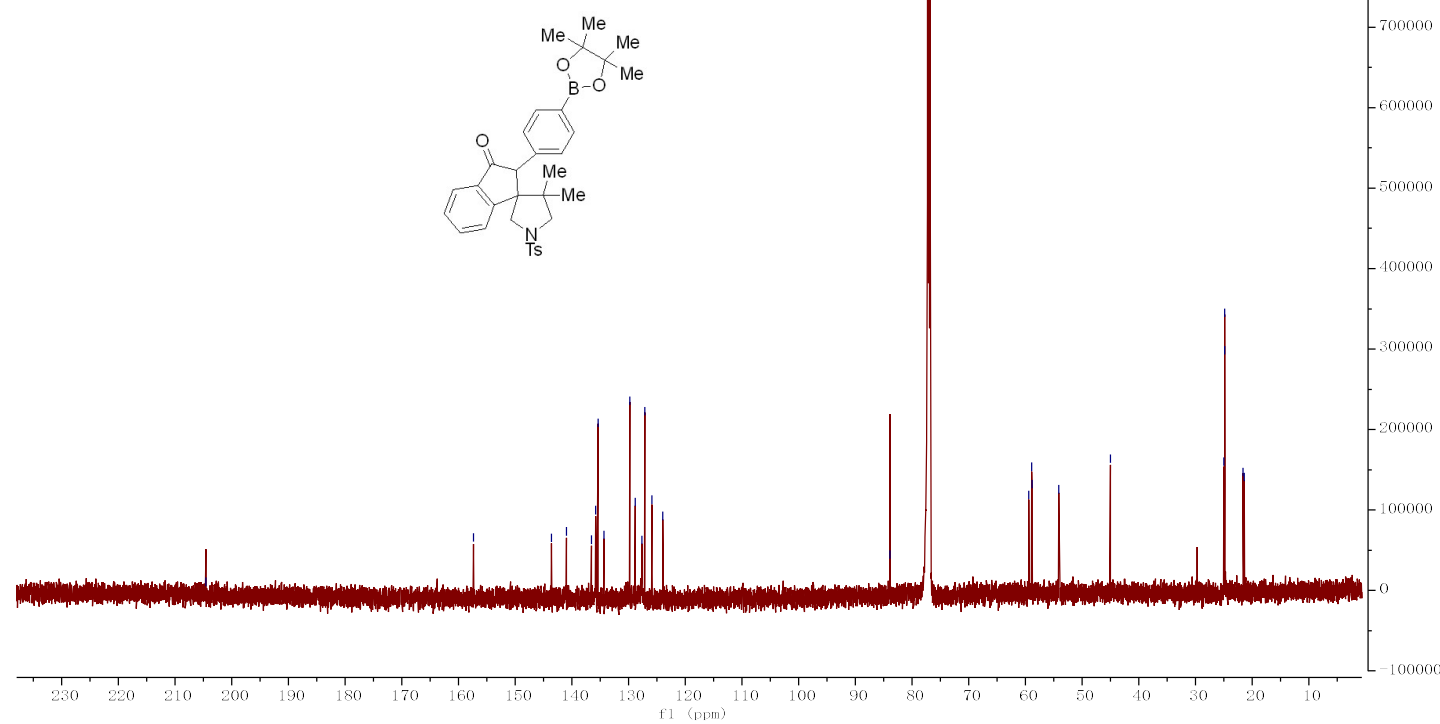


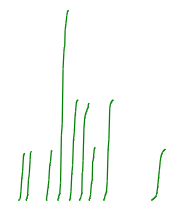

ininim
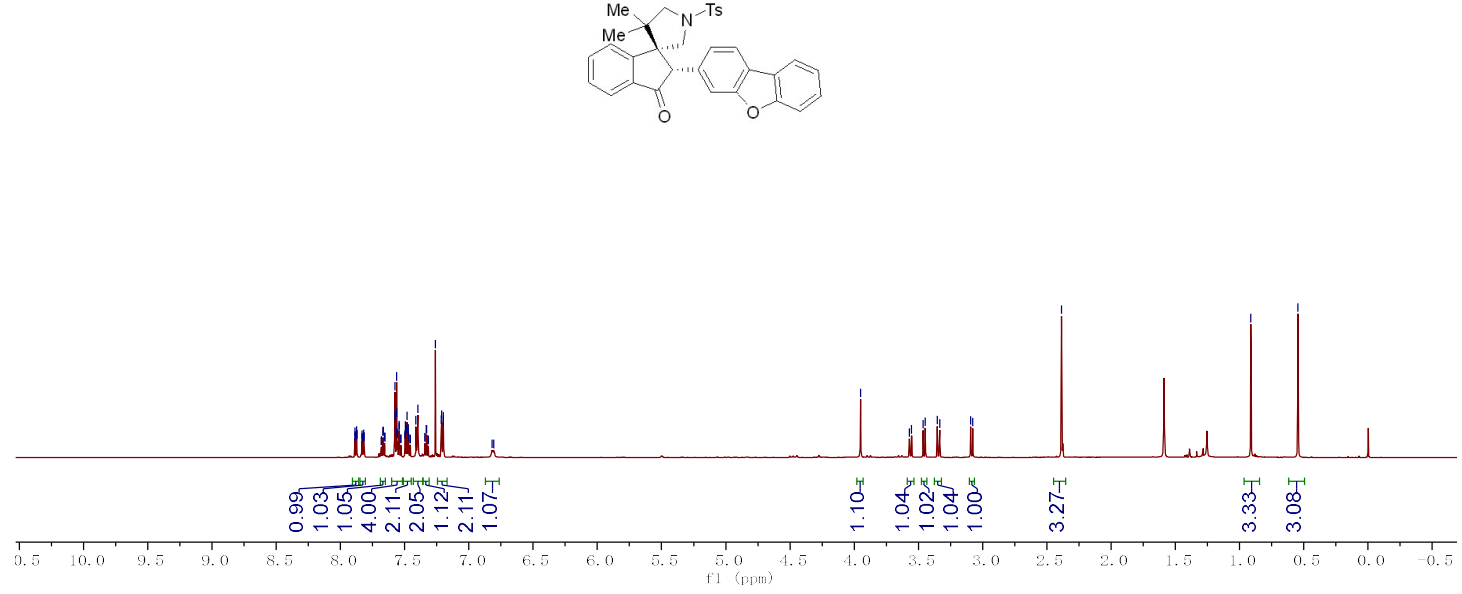

\&

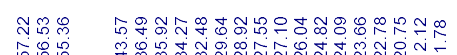

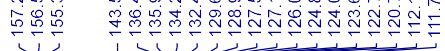

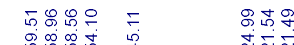

i

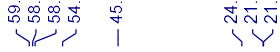
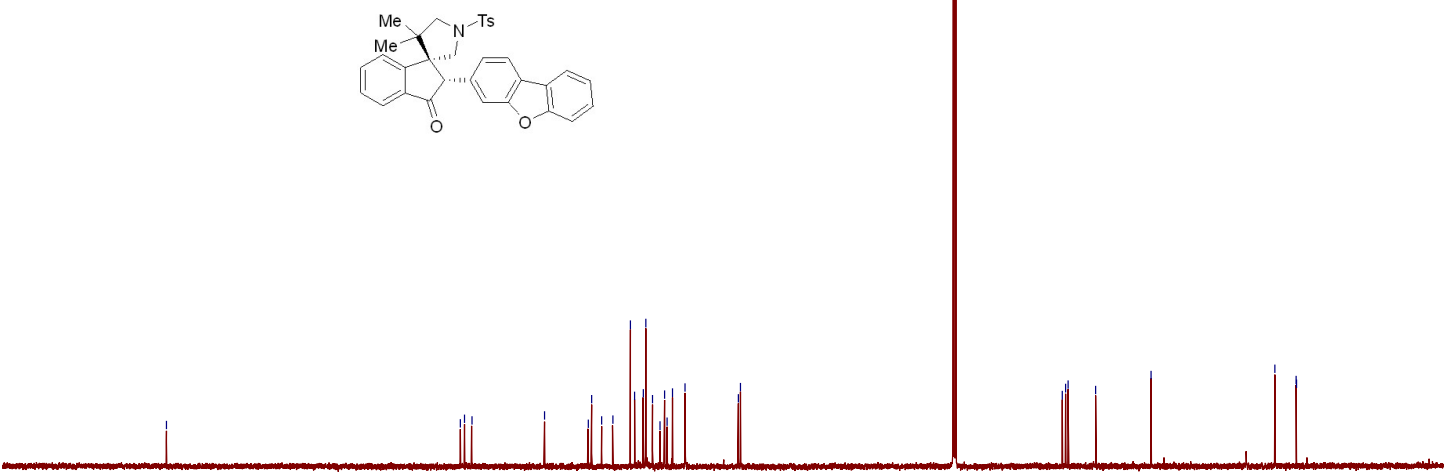

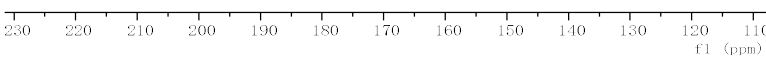


3j

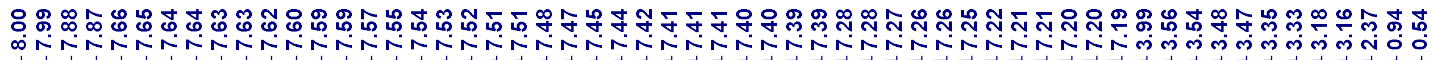
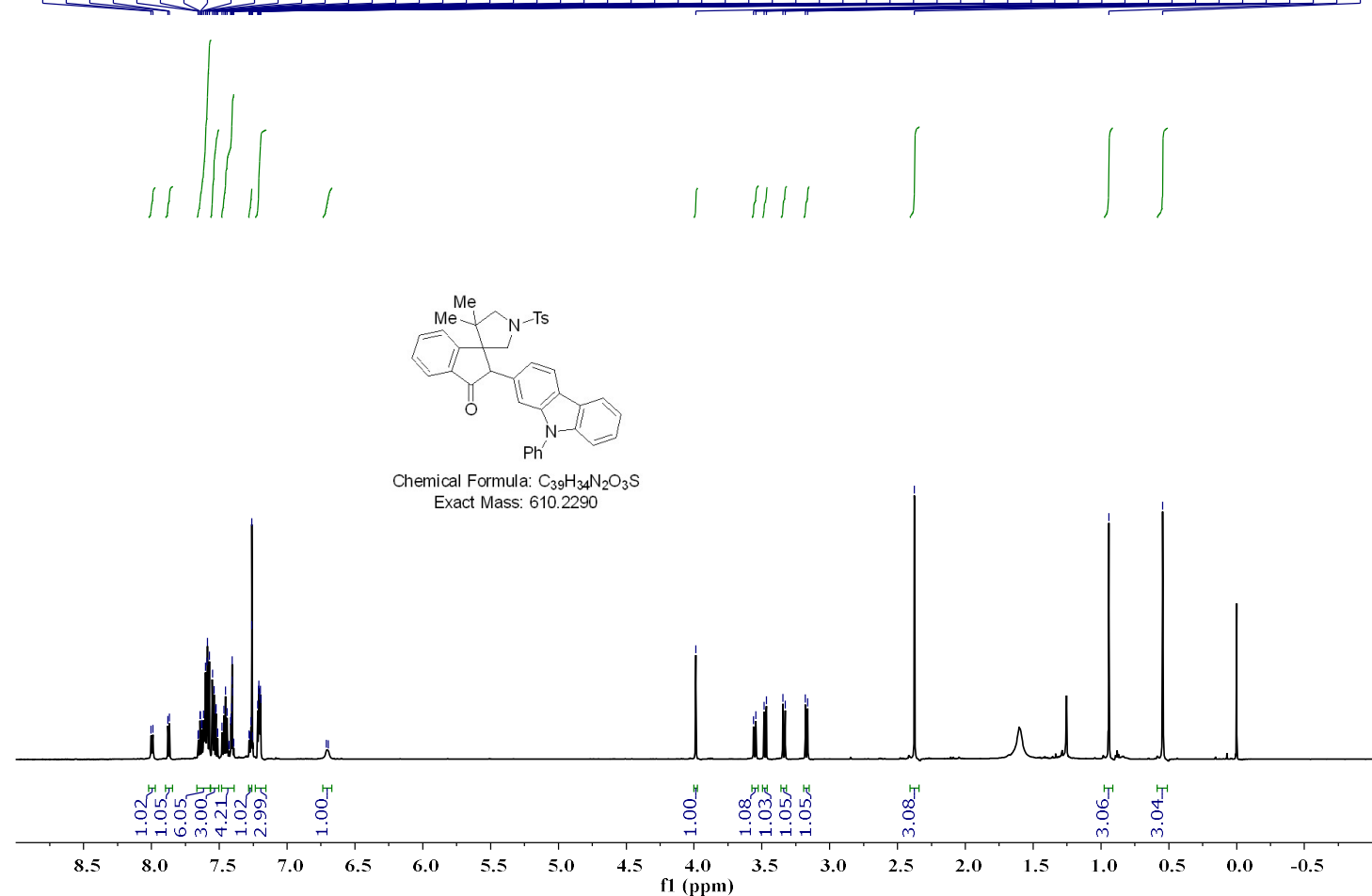

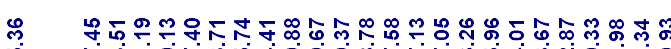

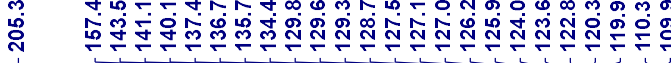

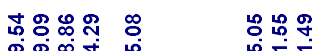

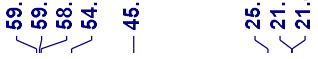

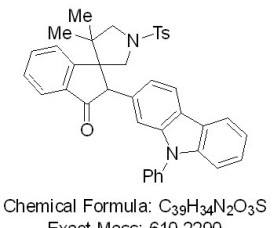

Exact Mass: 610.2290

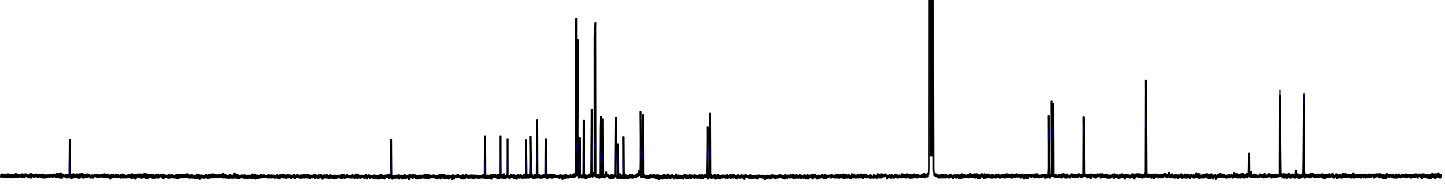

$\begin{array}{llllllllllll}210 & 200 & 190 & 180 & 170 & 160 & 150 & 140 & 130 & 120 & 110 & 100 \\ \mathrm{f} 1(\mathrm{ppm})\end{array}$ 


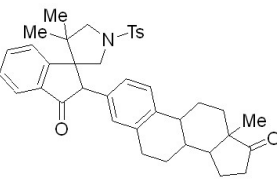

Chemical Formula: $\mathrm{C}_{39} \mathrm{H}_{43} \mathrm{NO}_{4} \mathrm{~S}$ Exact Mass: 621.2913
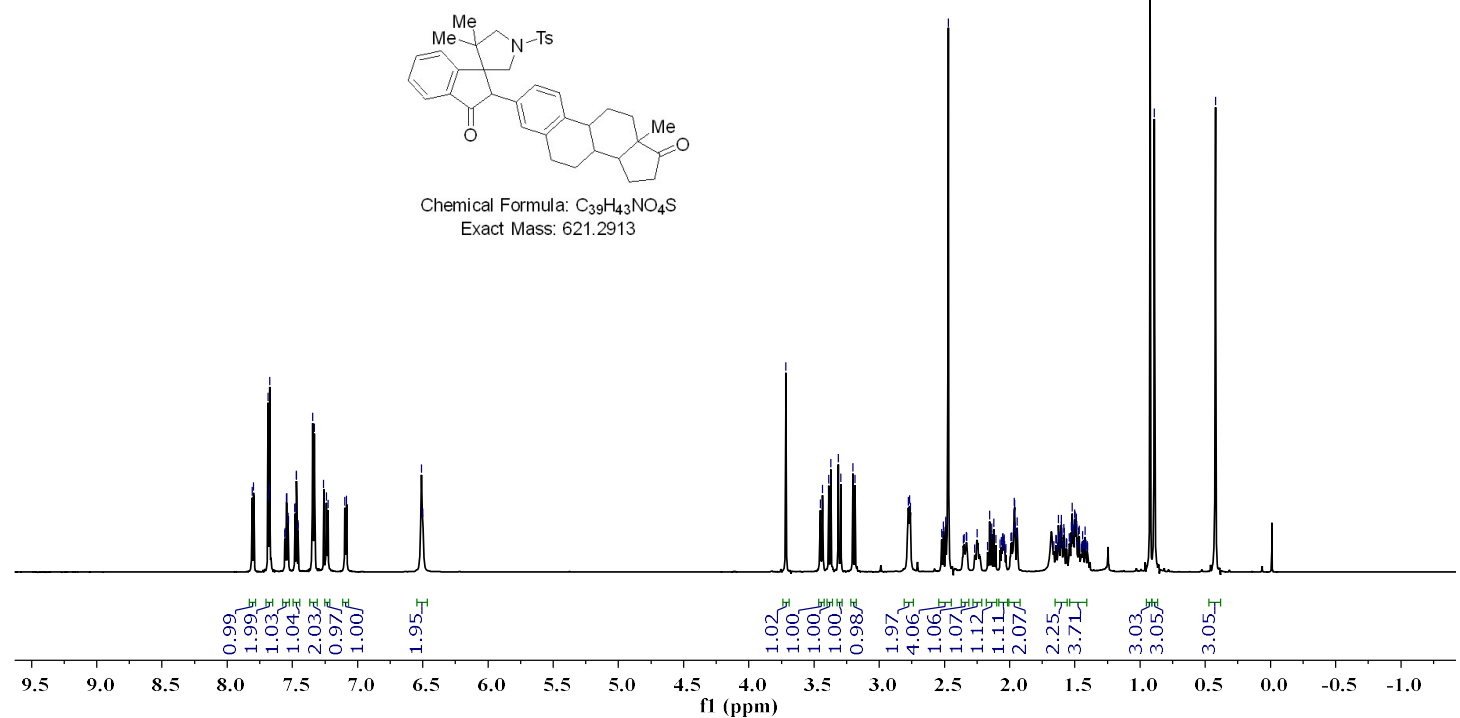

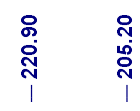

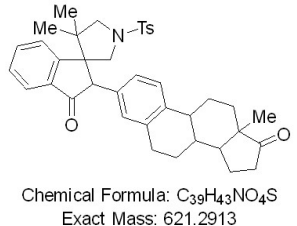




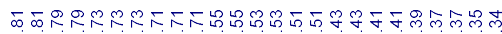

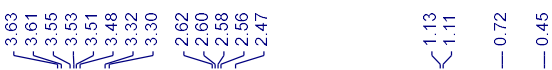

$\int 1 / 1$

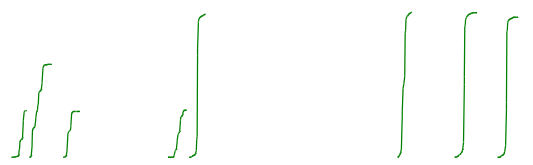

(ne

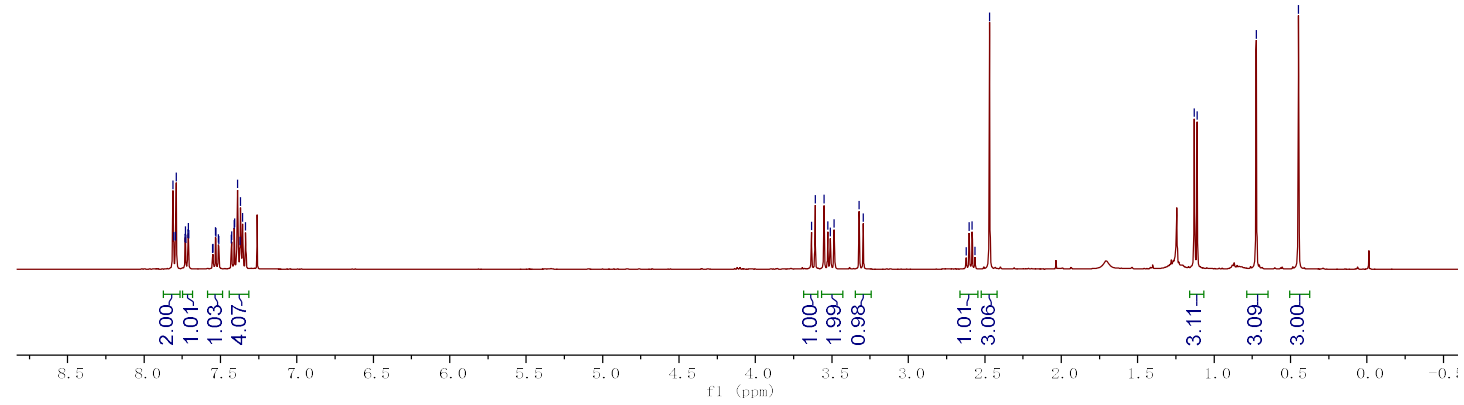

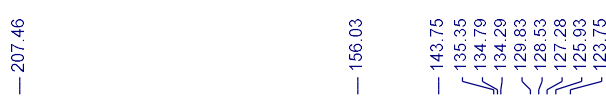

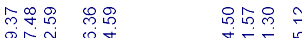

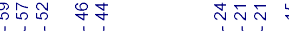

(Ne

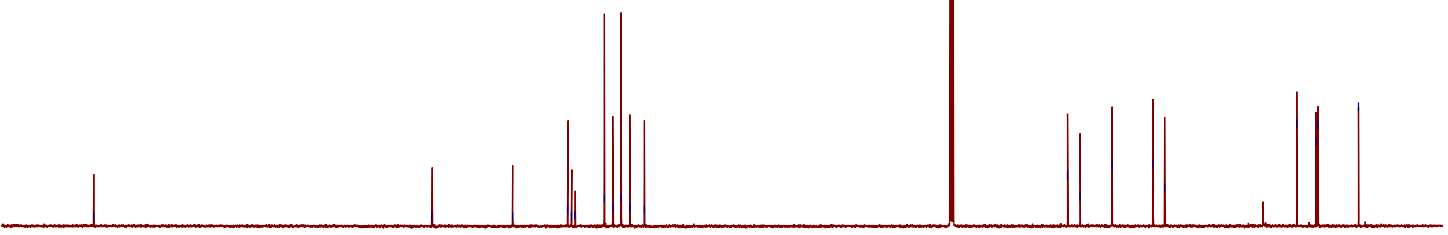

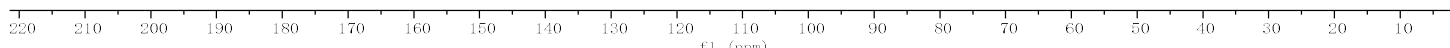


$3 m$

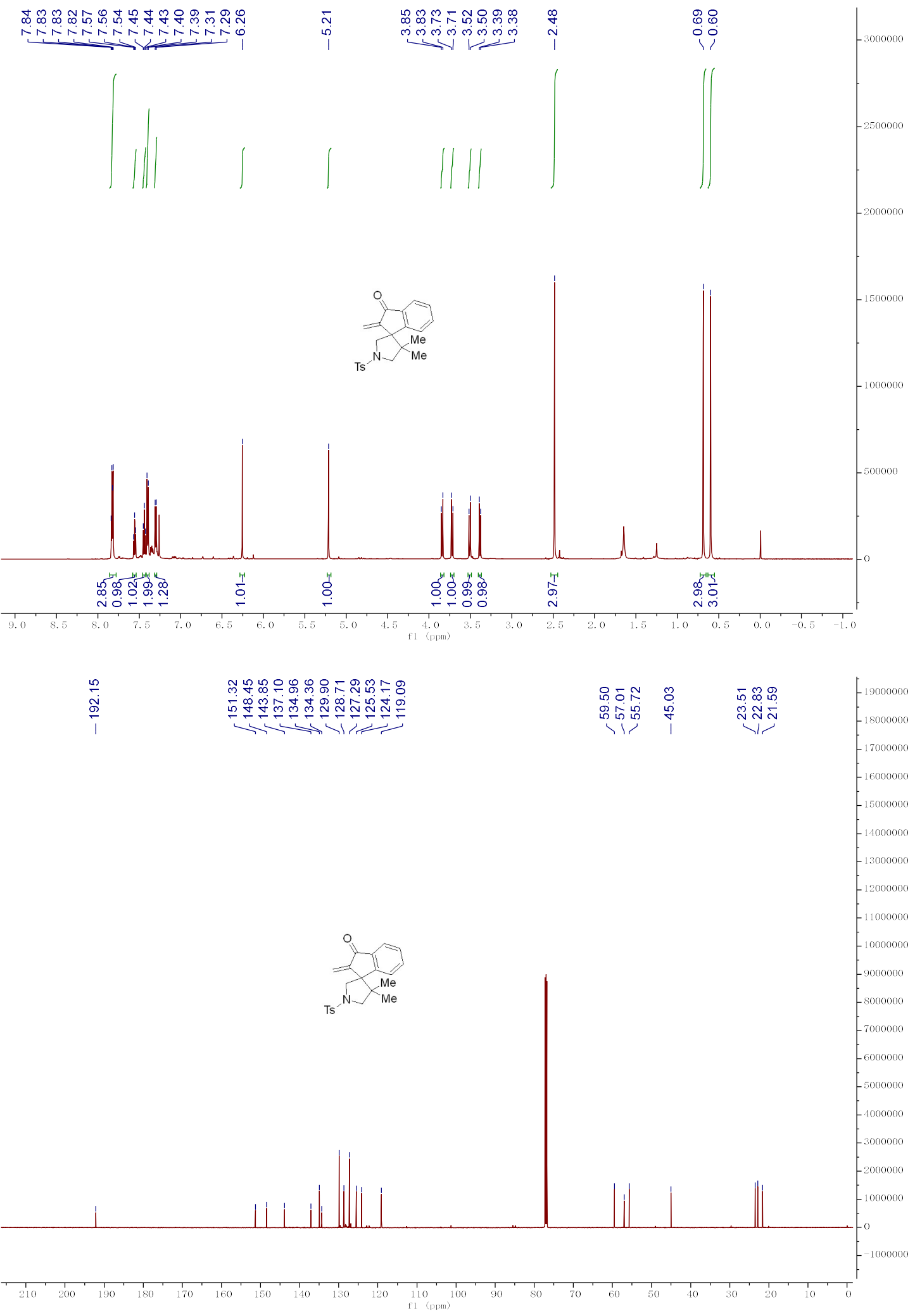


$3 n$
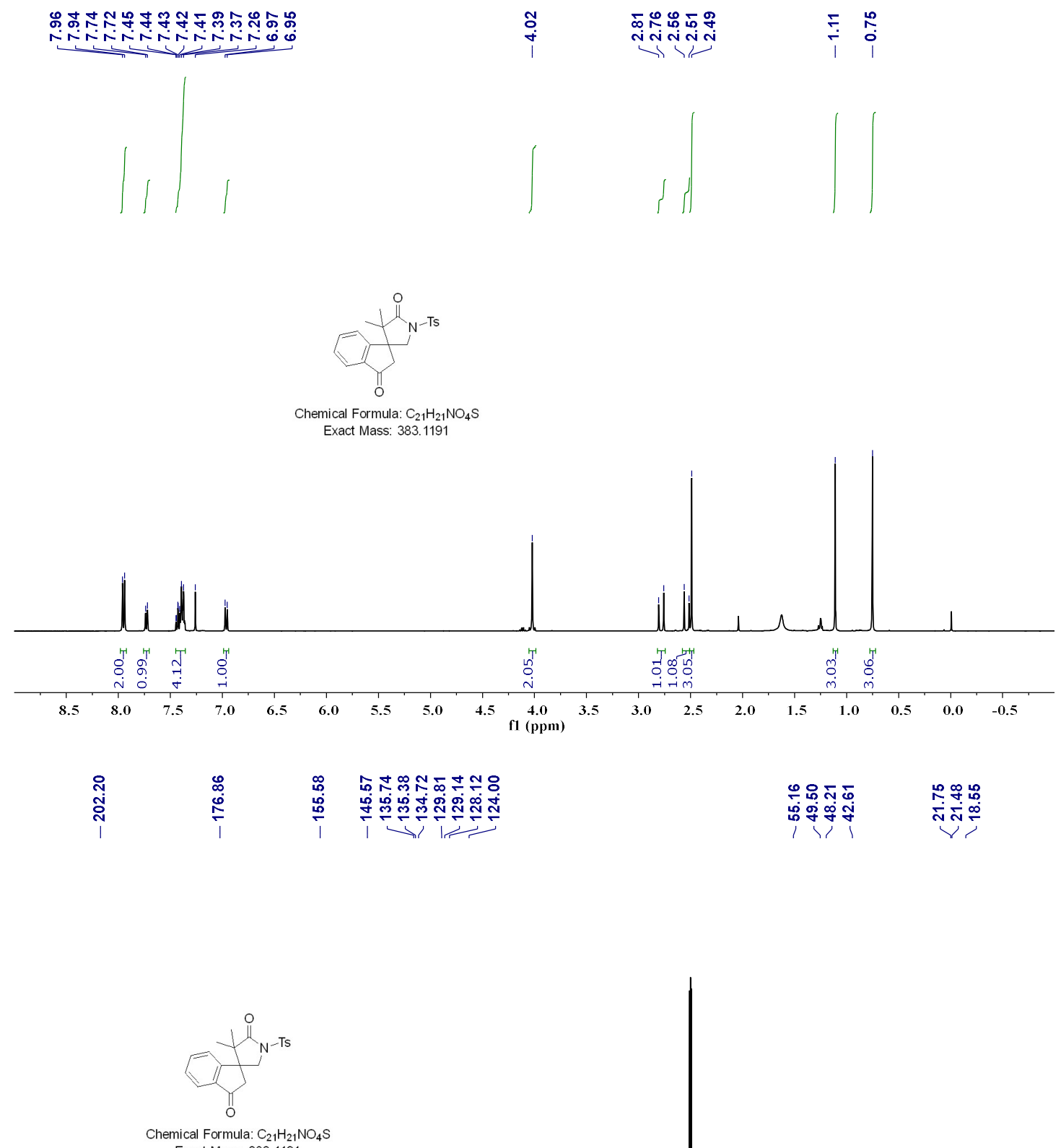

Exact Mass: 383.1191

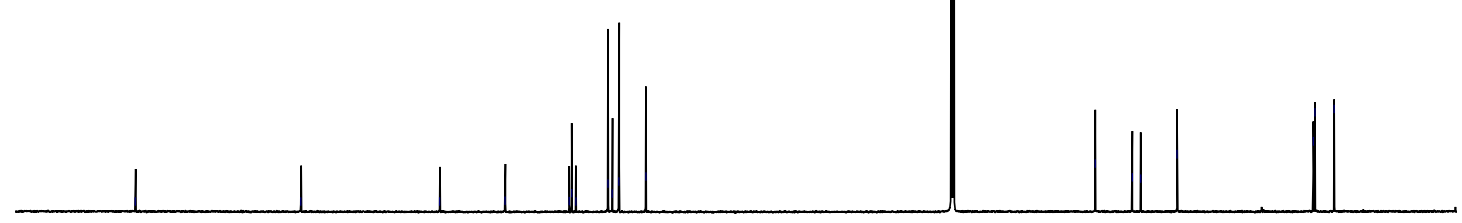

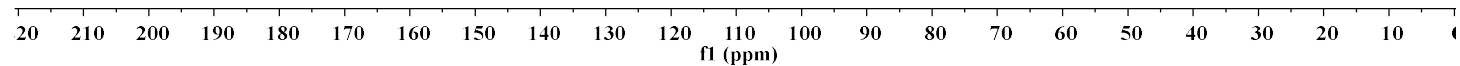




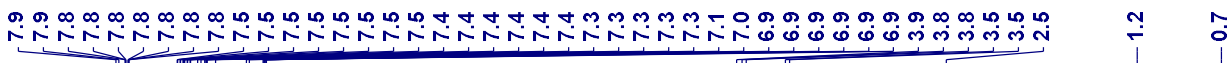
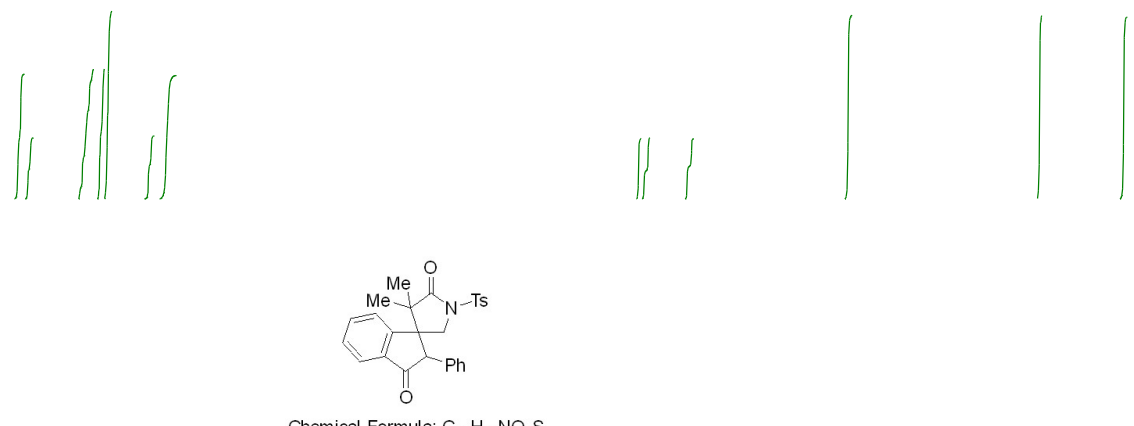

Chemical Formula: $\mathrm{C}_{27} \mathrm{H}_{25} \mathrm{NO}_{4}$

Exact Mass: 459.1504

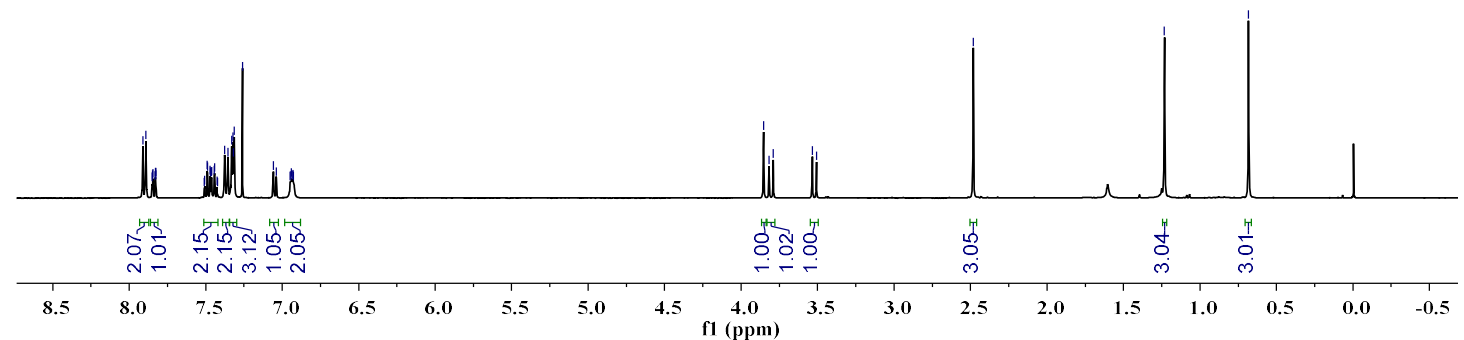

至

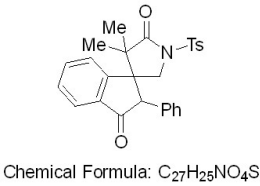

Exact Mass: 459.1504

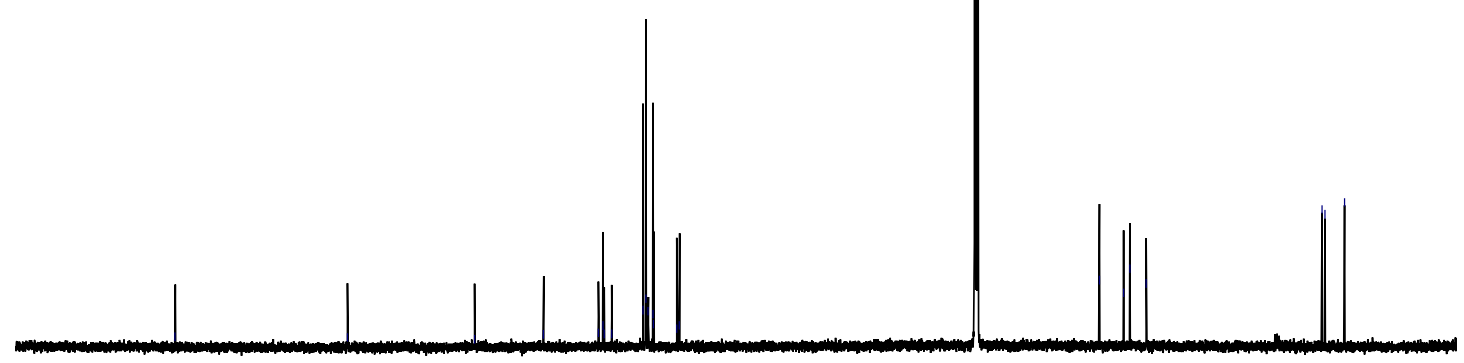

$\begin{array}{llllllllllllllllllllll}220 & 210 & 200 & 190 & 180 & 170 & 160 & 150 & 140 & 130 & \underset{120}{120}\left(\begin{array}{ll}110 \\ \mathrm{f} 1(\mathrm{pmm})\end{array}\right. & 100 & 90 & 80 & 70 & 60 & 50 & 40 & 30 & 20 & 10\end{array}$ 


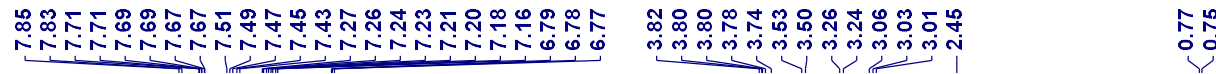
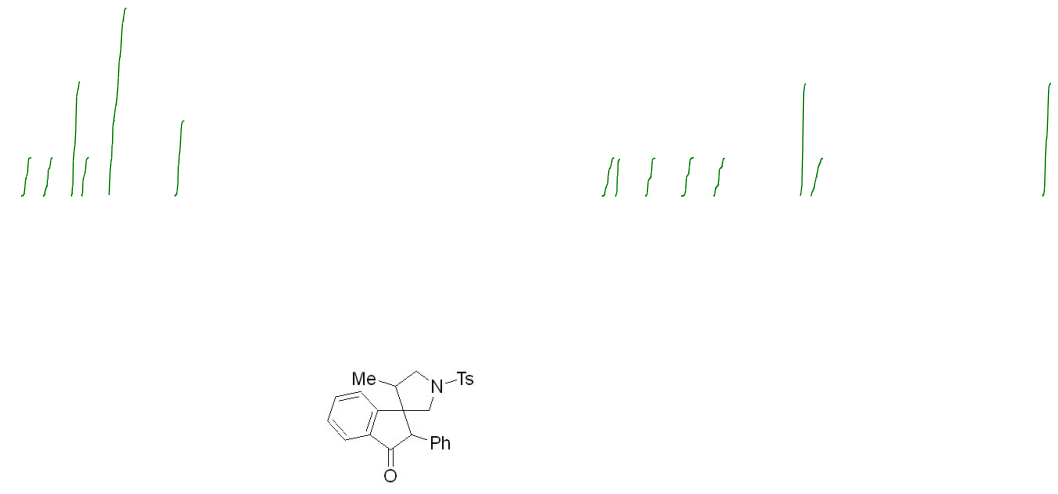

Chemical Formula: $\mathrm{C}_{26} \mathrm{H}_{25} \mathrm{NO}_{3} \mathrm{~S}$

Exact Mass: 431. 1555
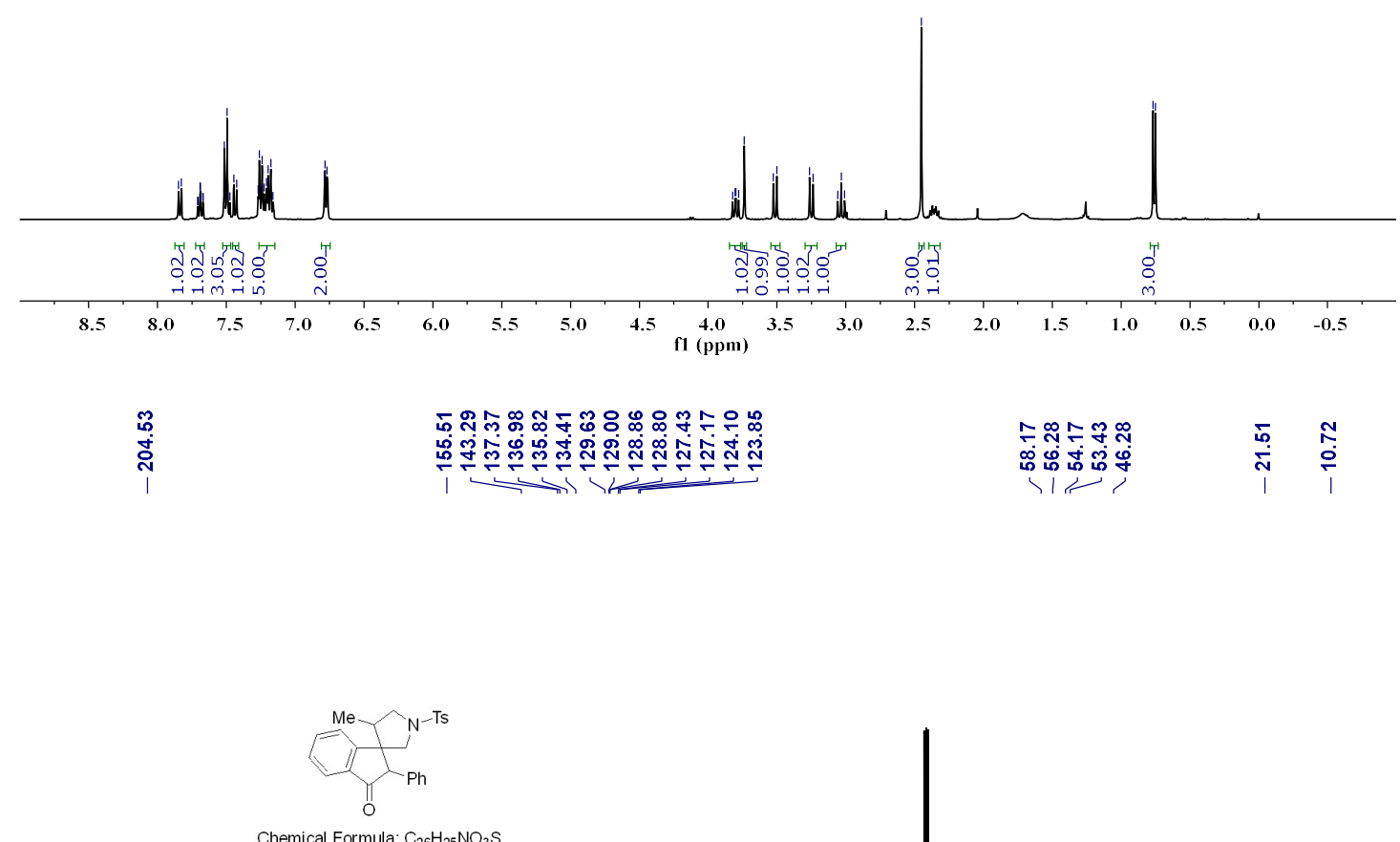

Exact Formula: $\mathrm{C}_{26} \mathrm{H}_{25} \mathrm{NO}$

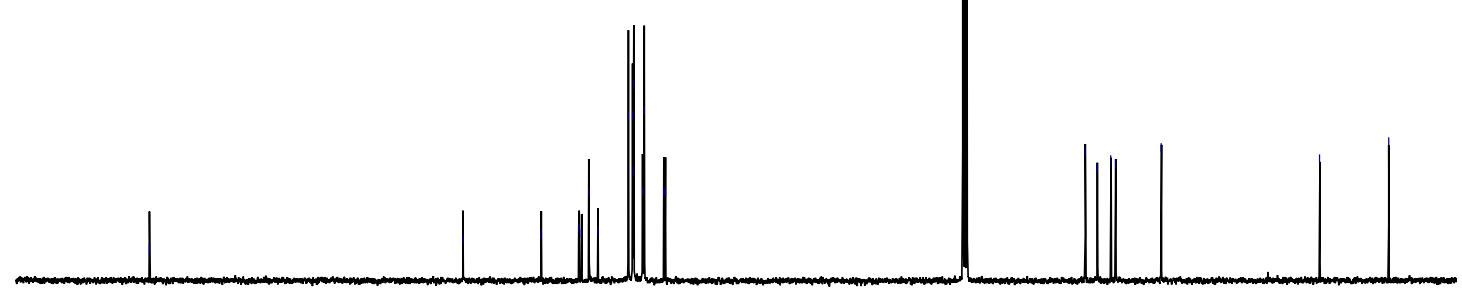

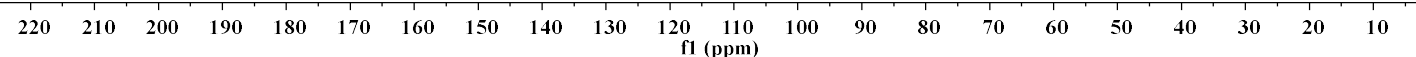


$3 q$

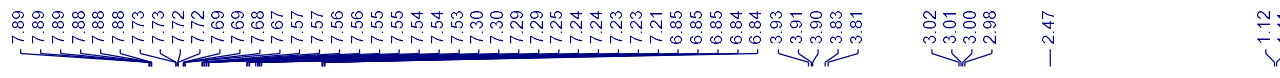

\|\|
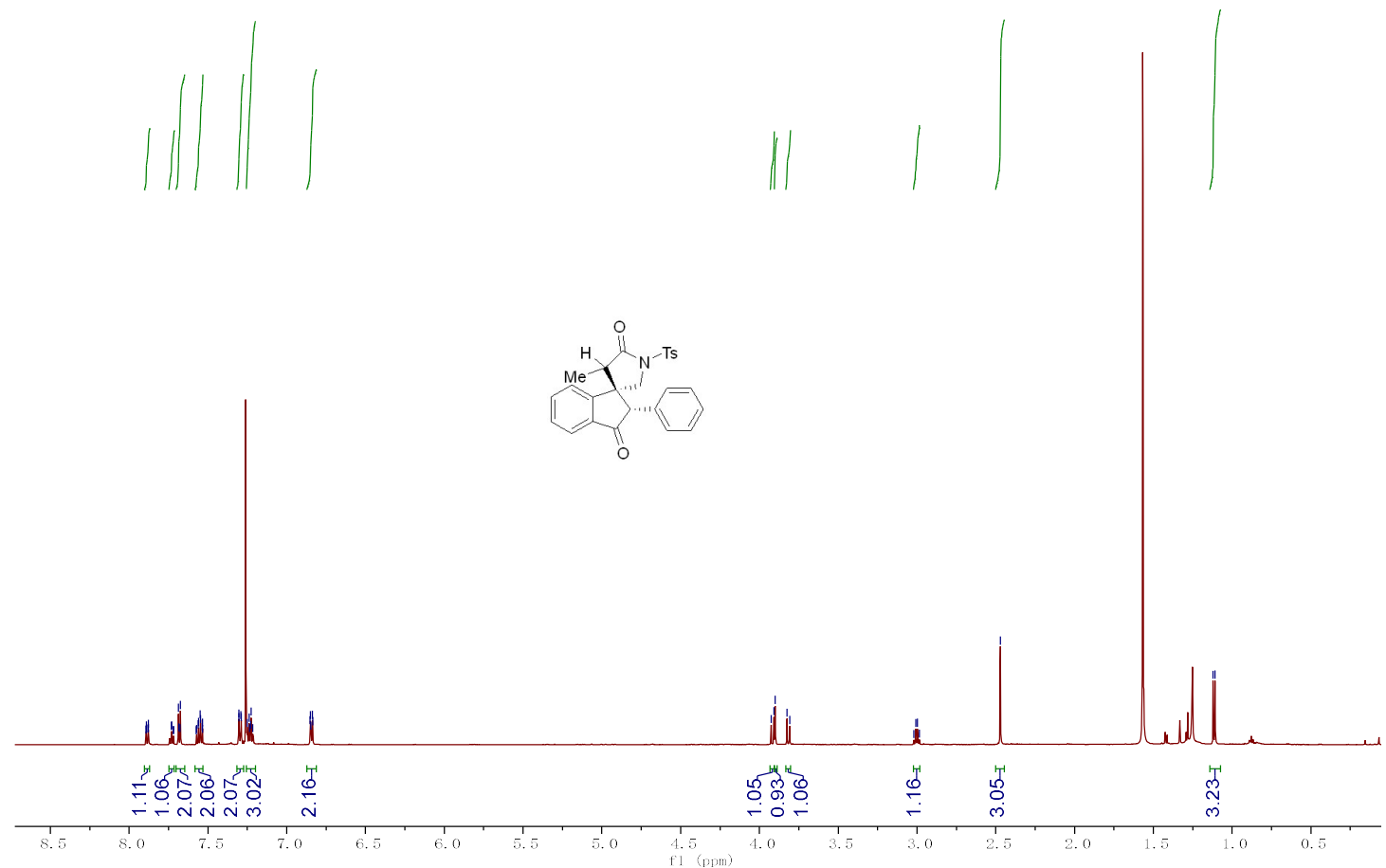

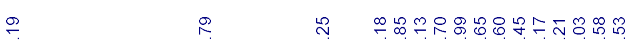

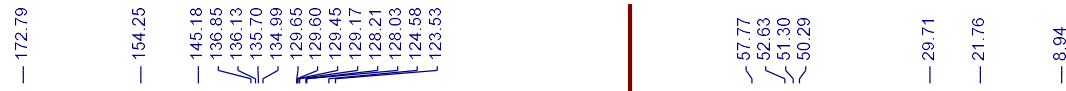

(1)
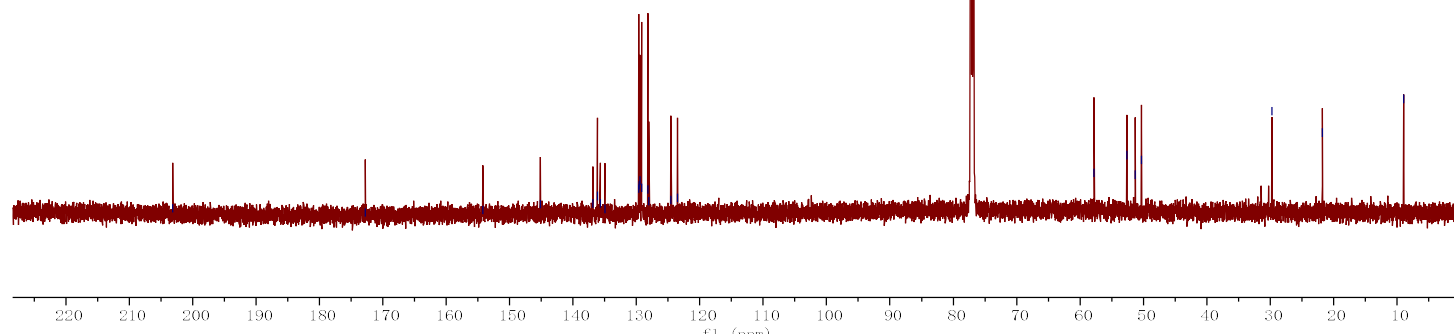


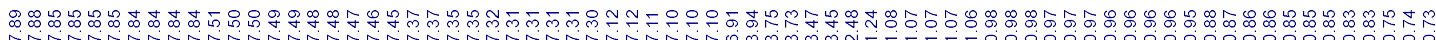
L
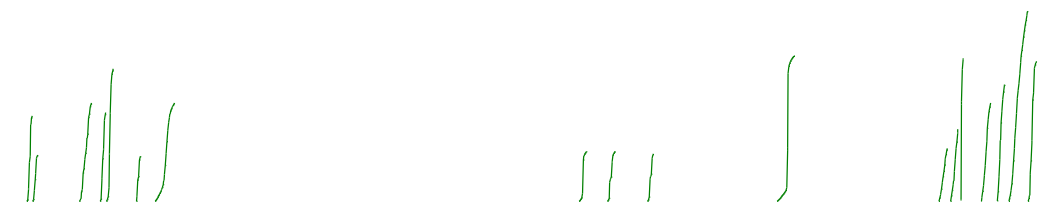

$$
\mathrm{Me}-\mathrm{O}_{\mathrm{O}}^{2}
$$

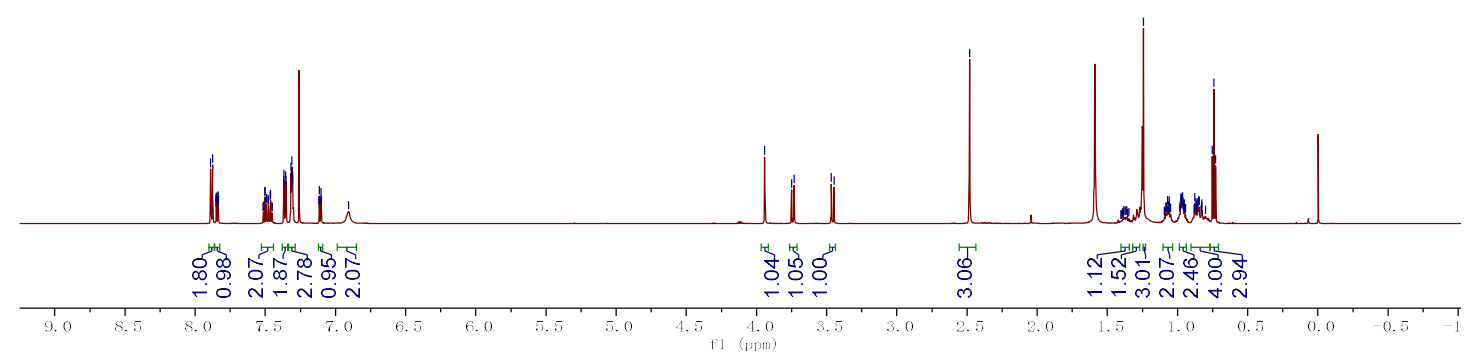

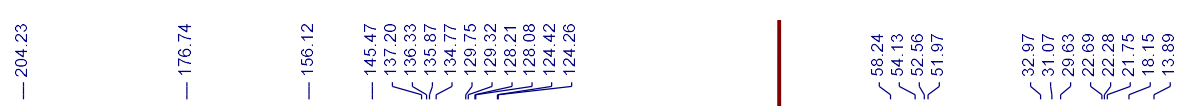$$
\mathrm{Me}-\underbrace{2}_{\mathrm{C}}
$$

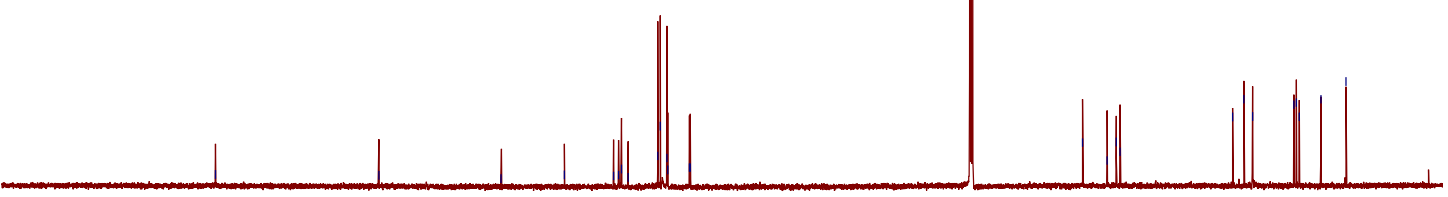

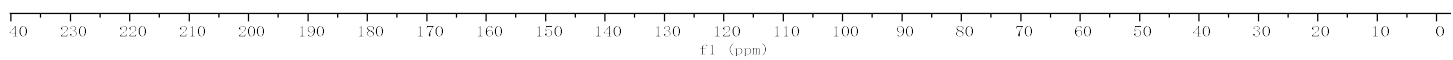


$3 s$

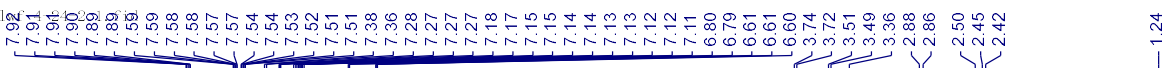
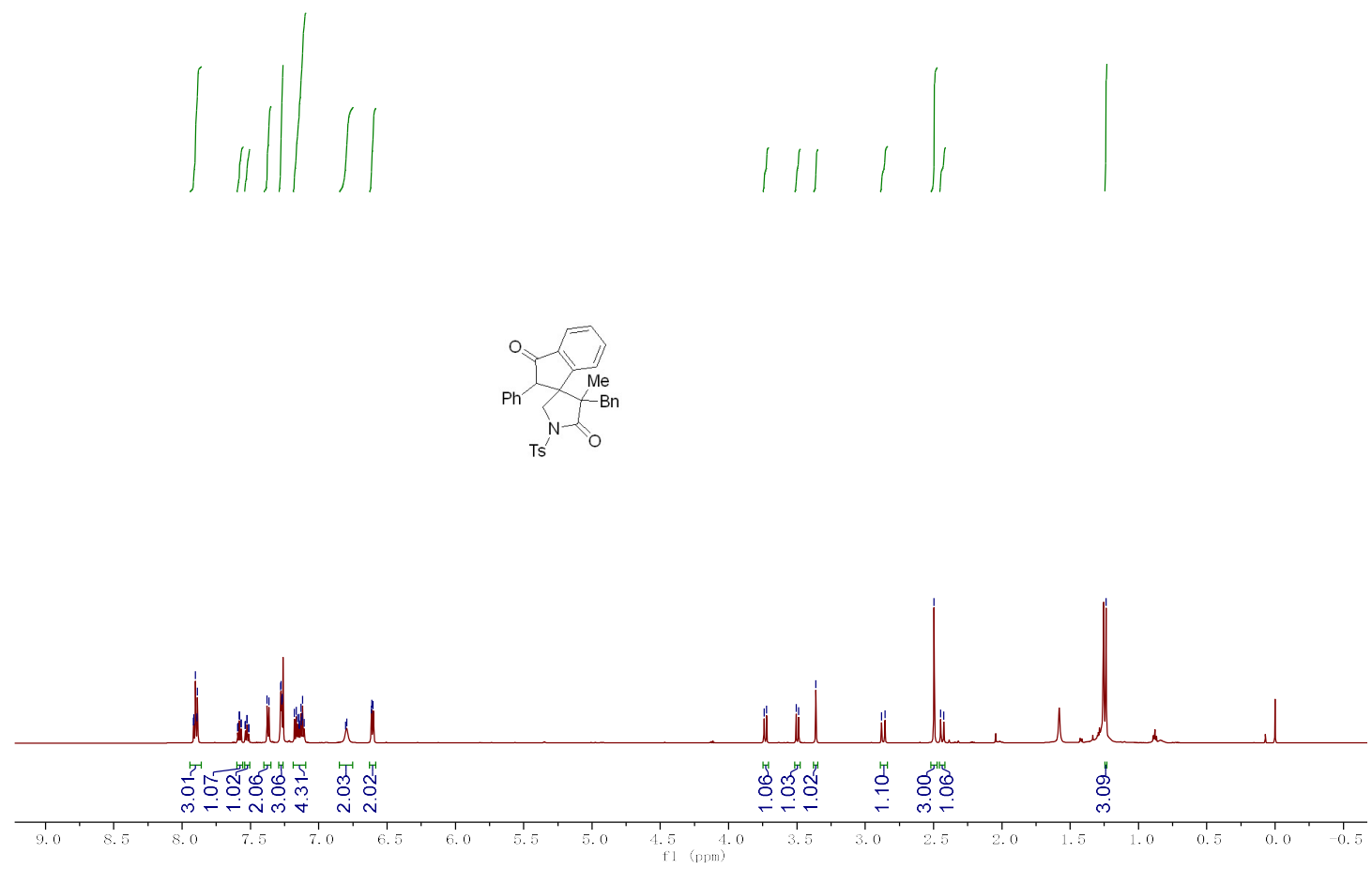

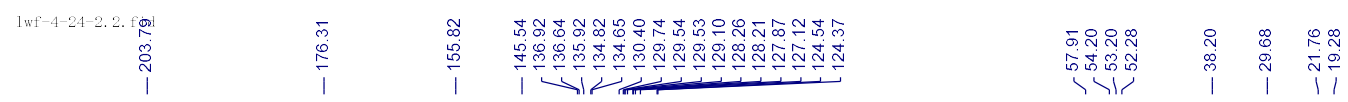
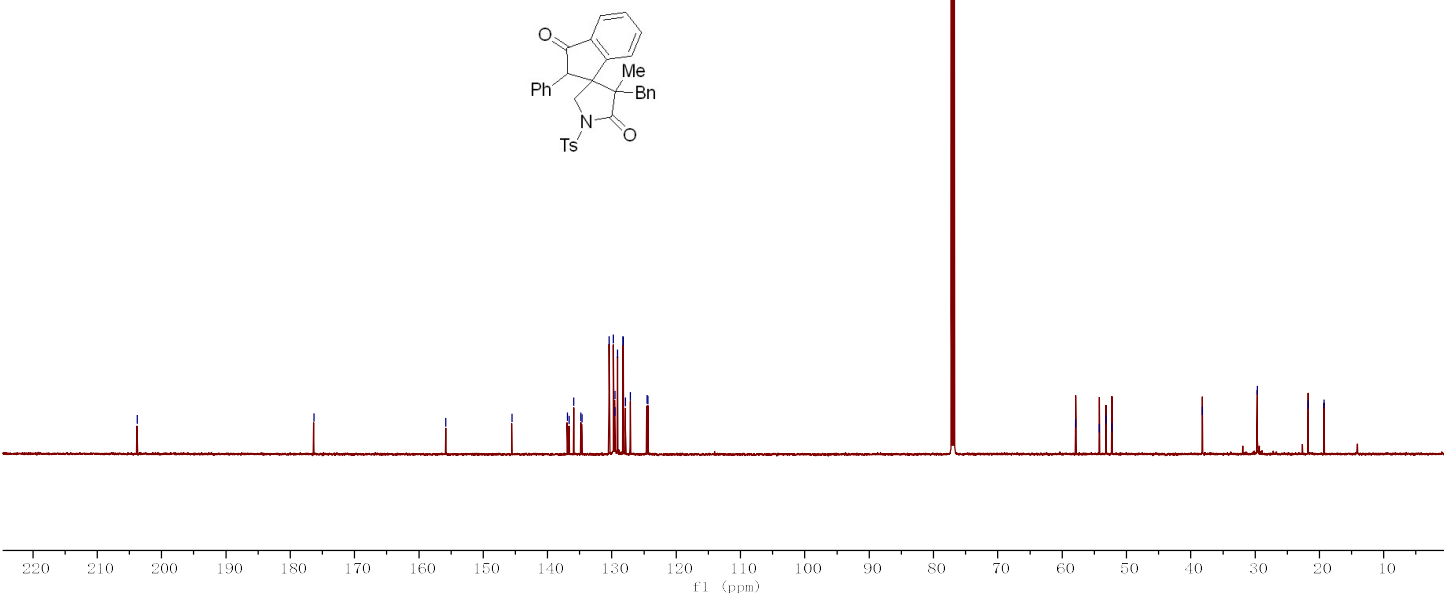


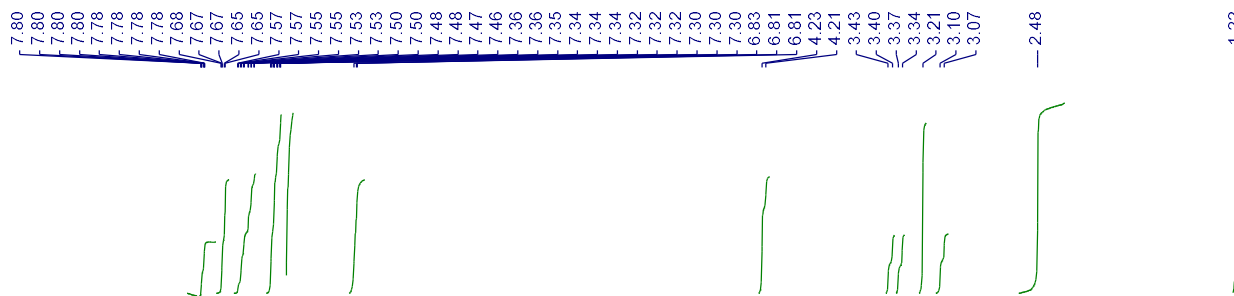

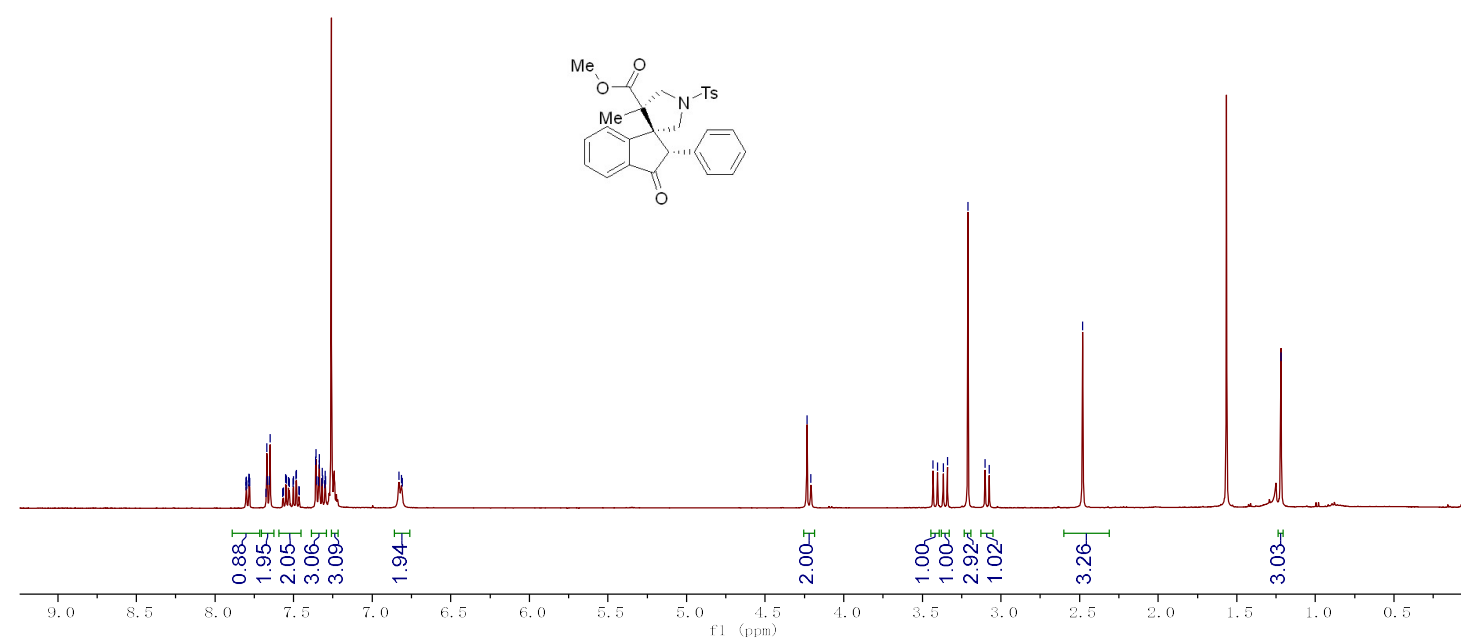

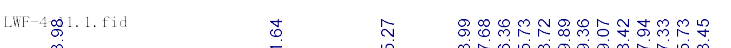

Me

(1)

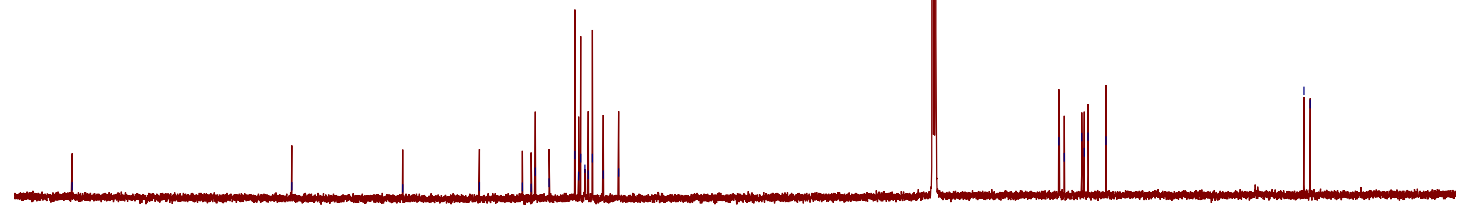

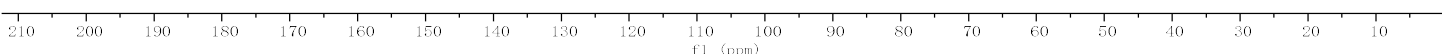




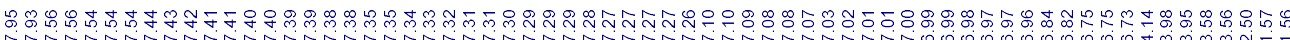

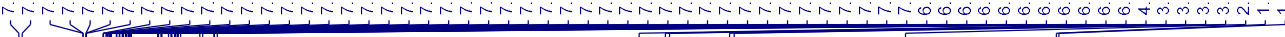
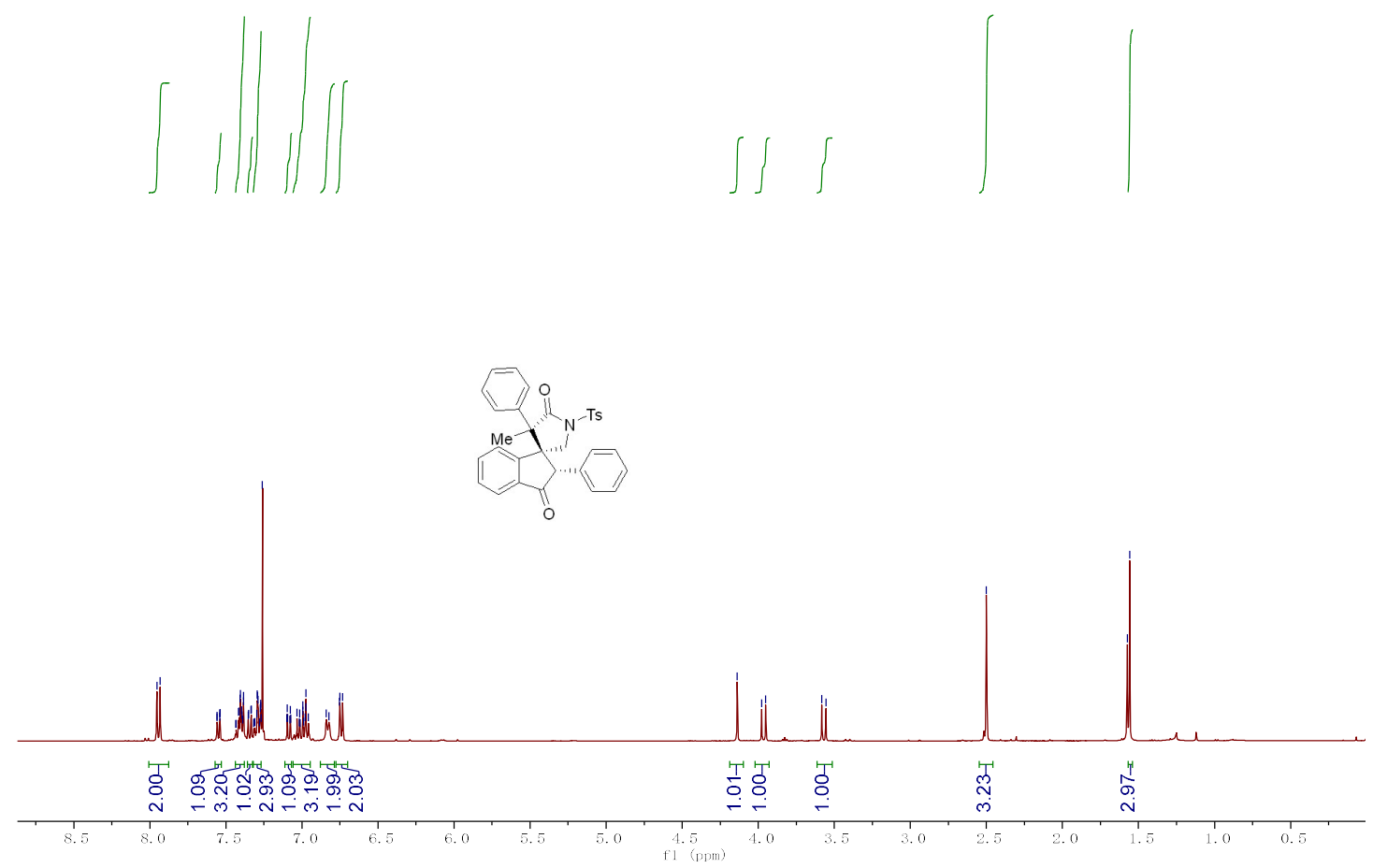

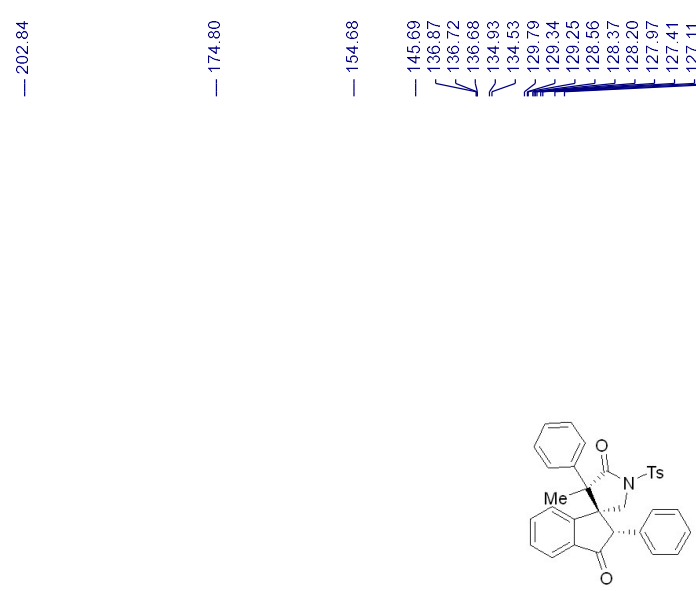
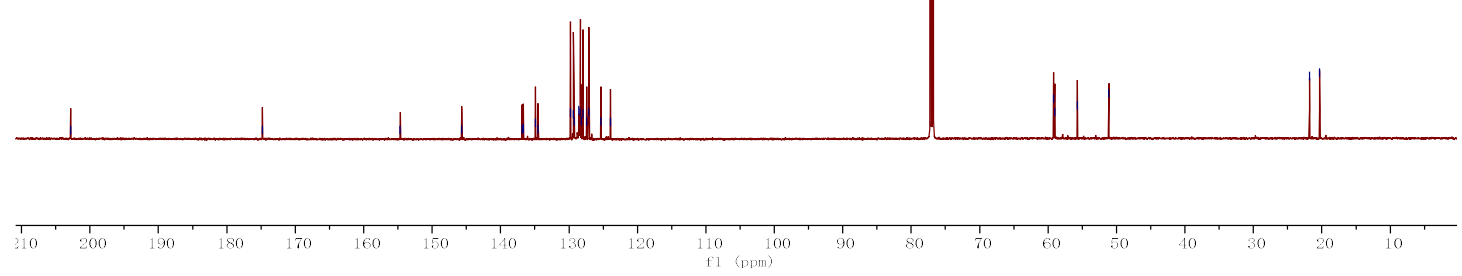


\section{$3 v$}

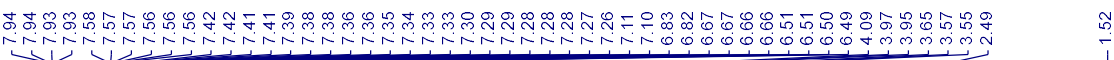
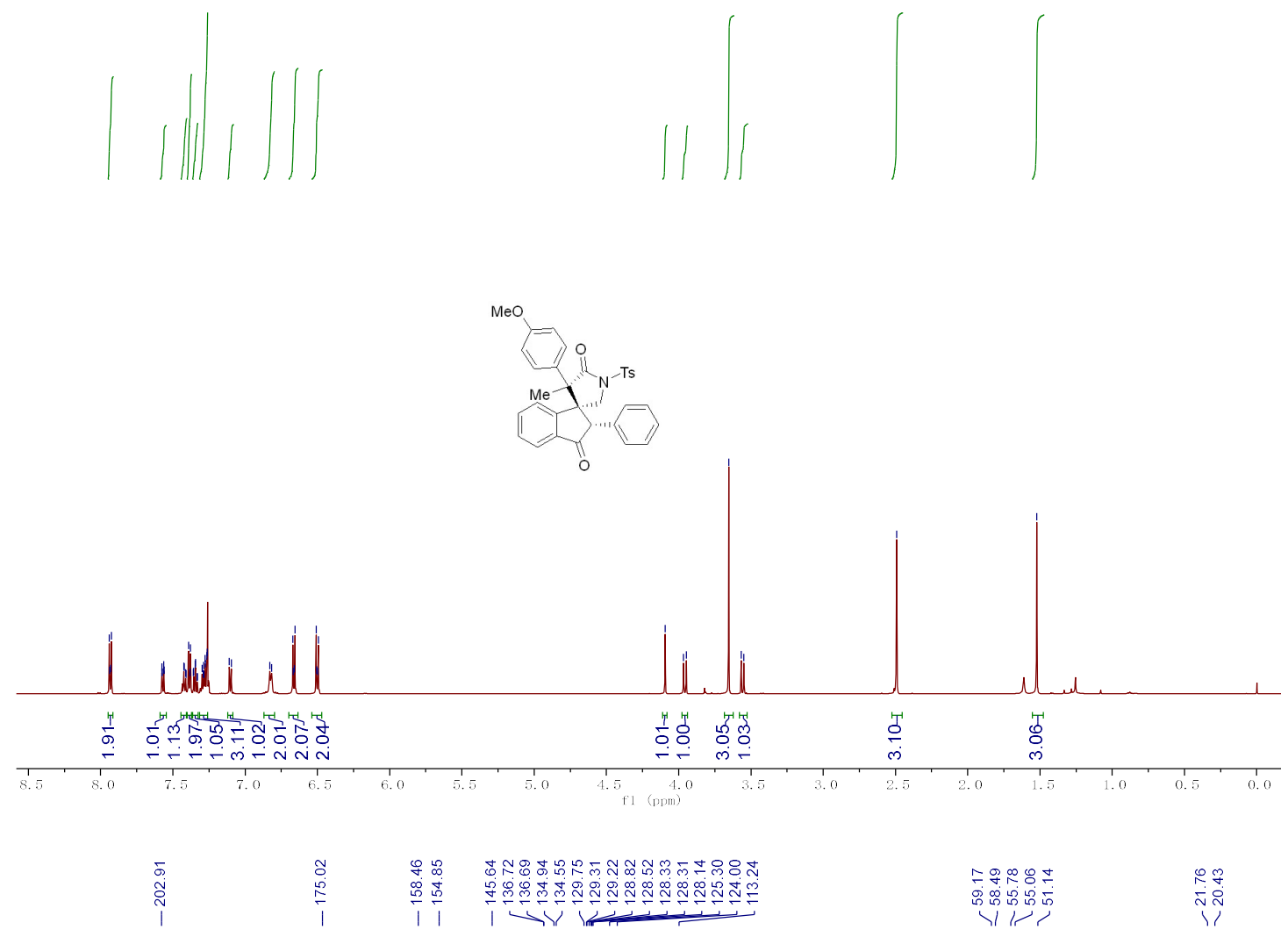

$\mathrm{MeO}$
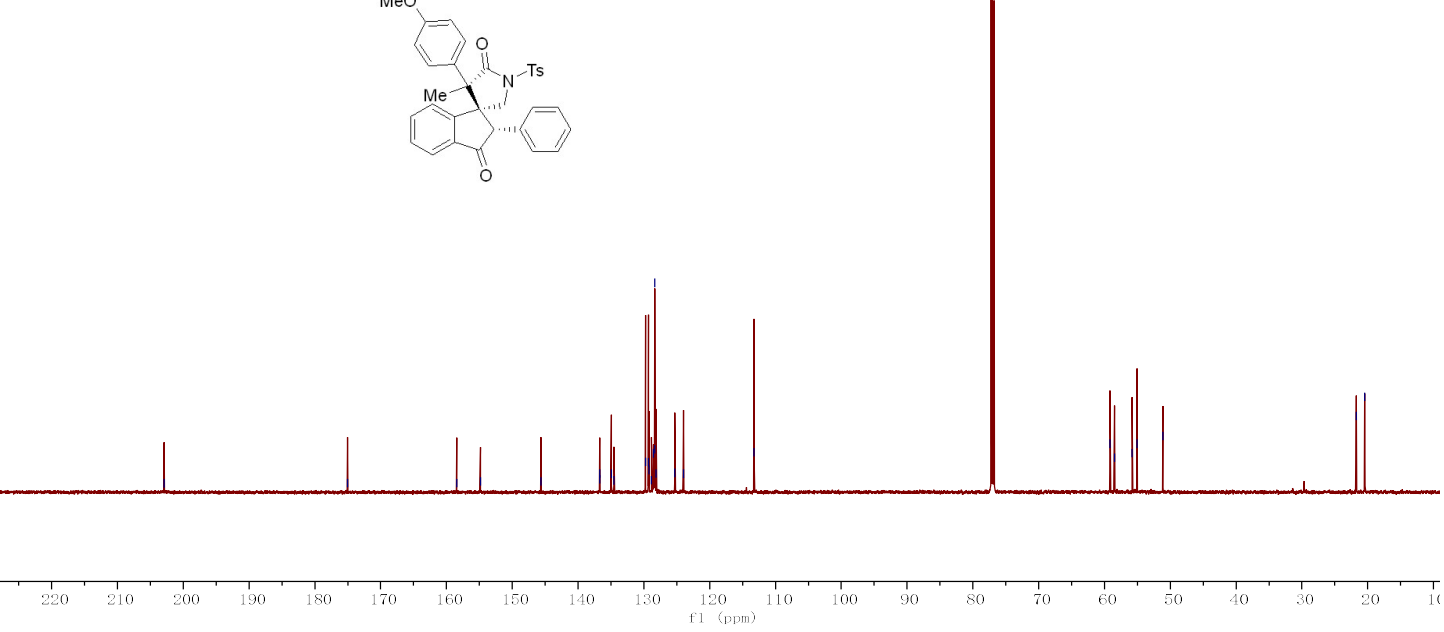
$3 w$

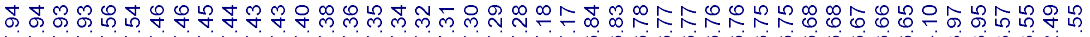

然
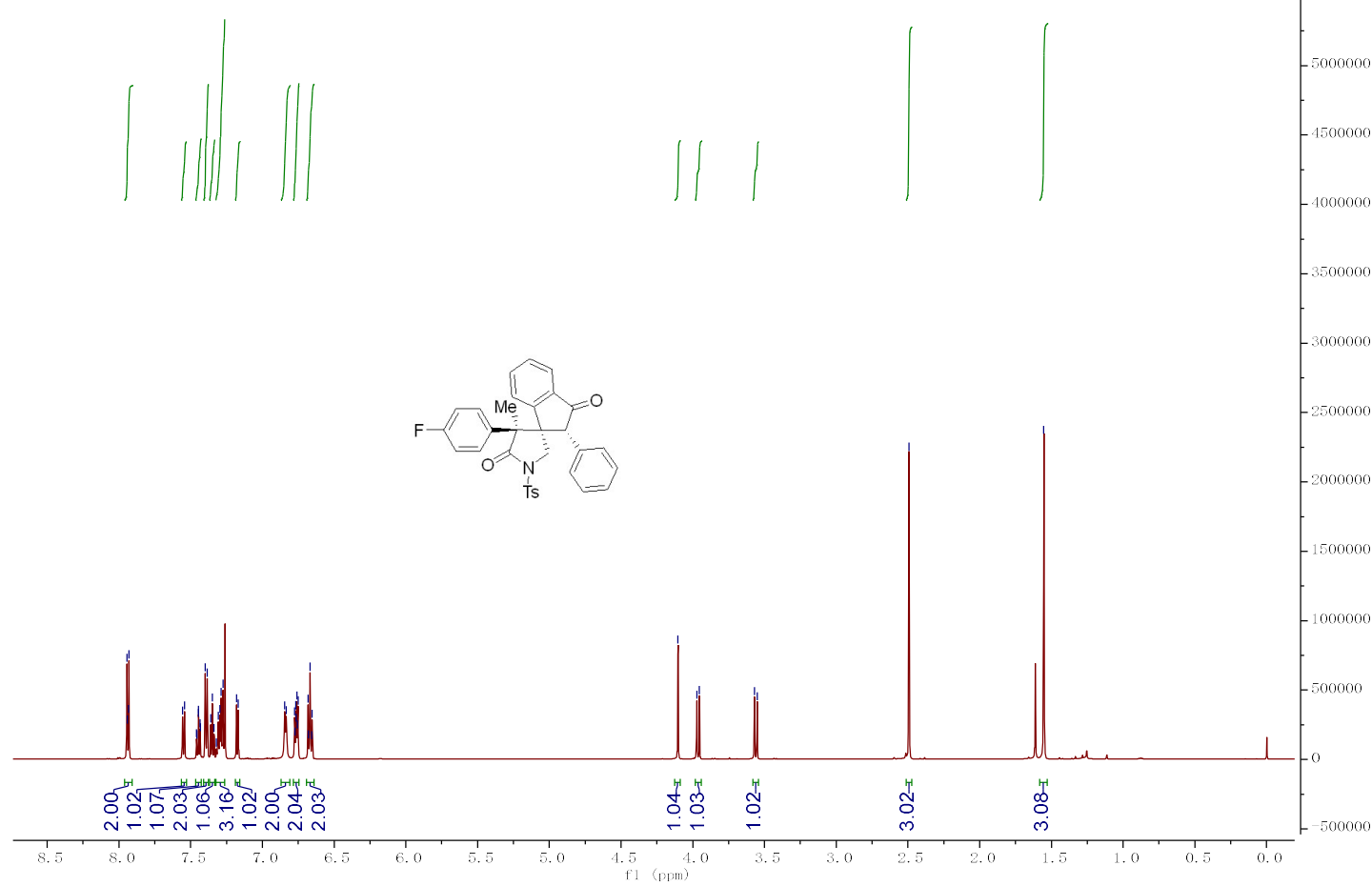

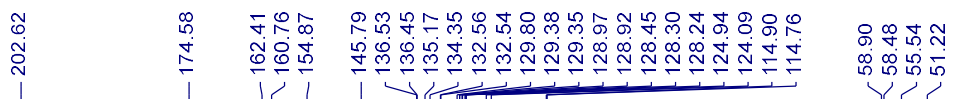

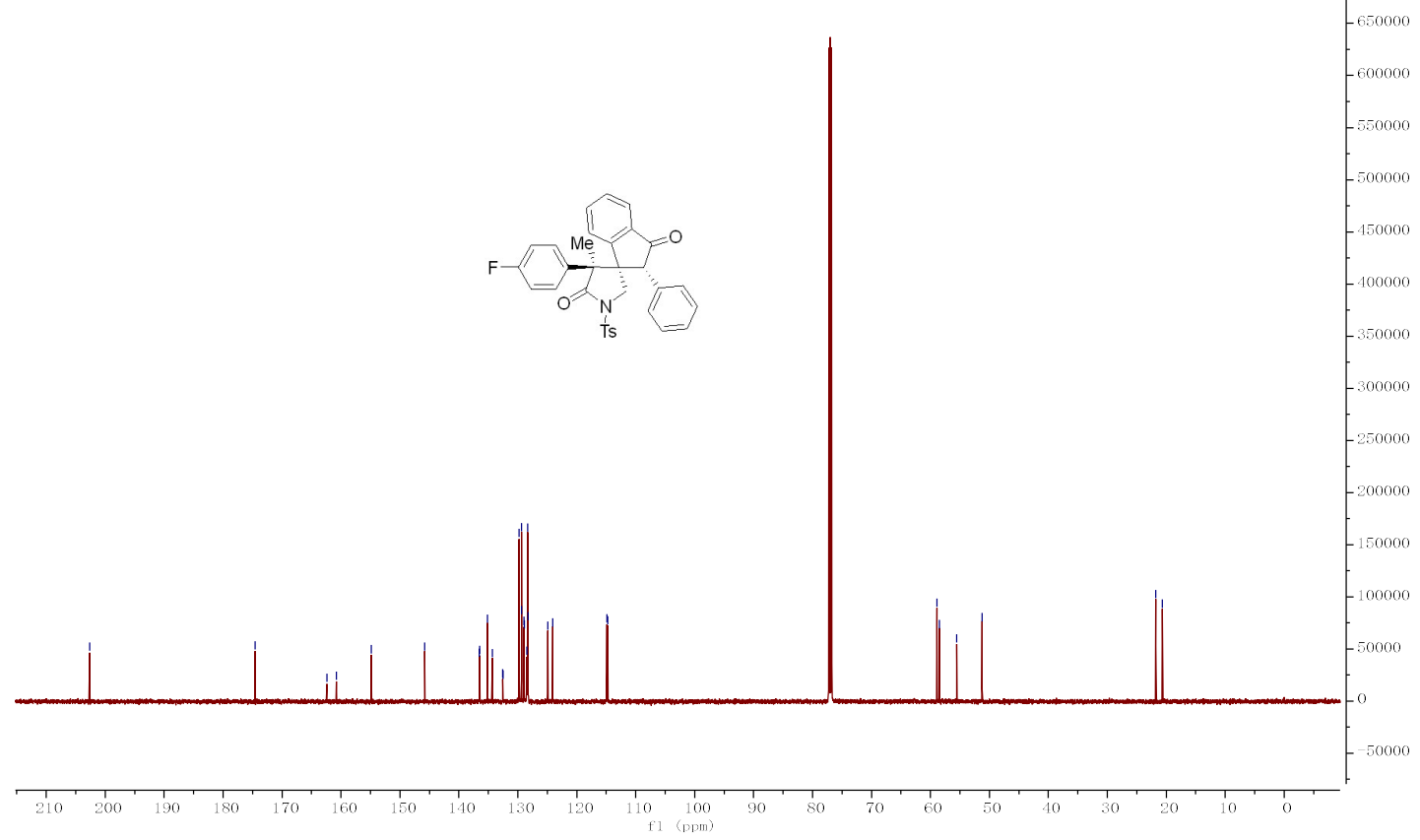




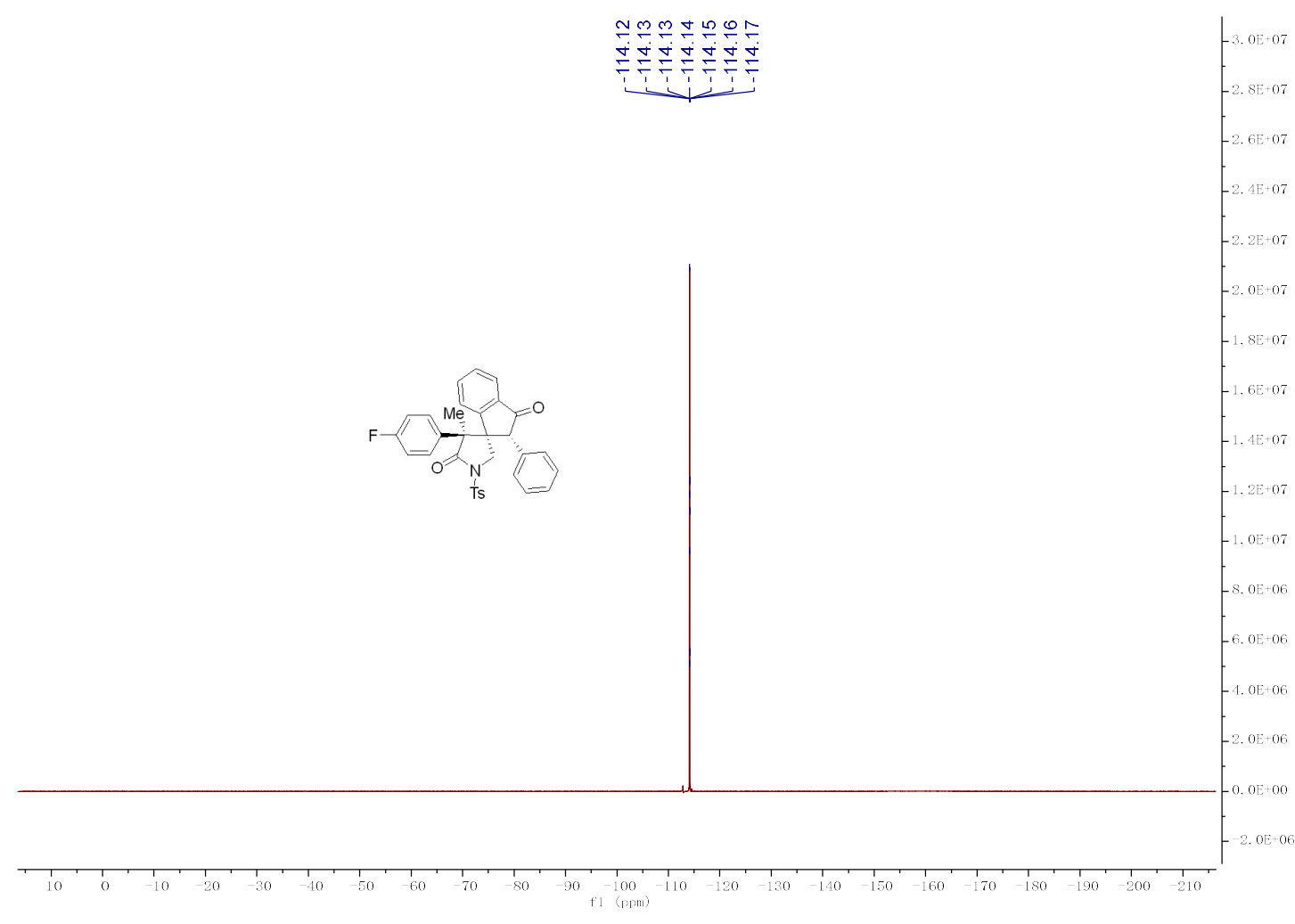




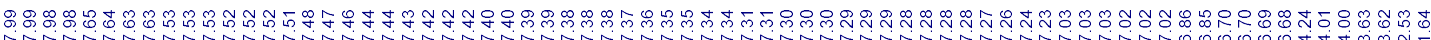
赑
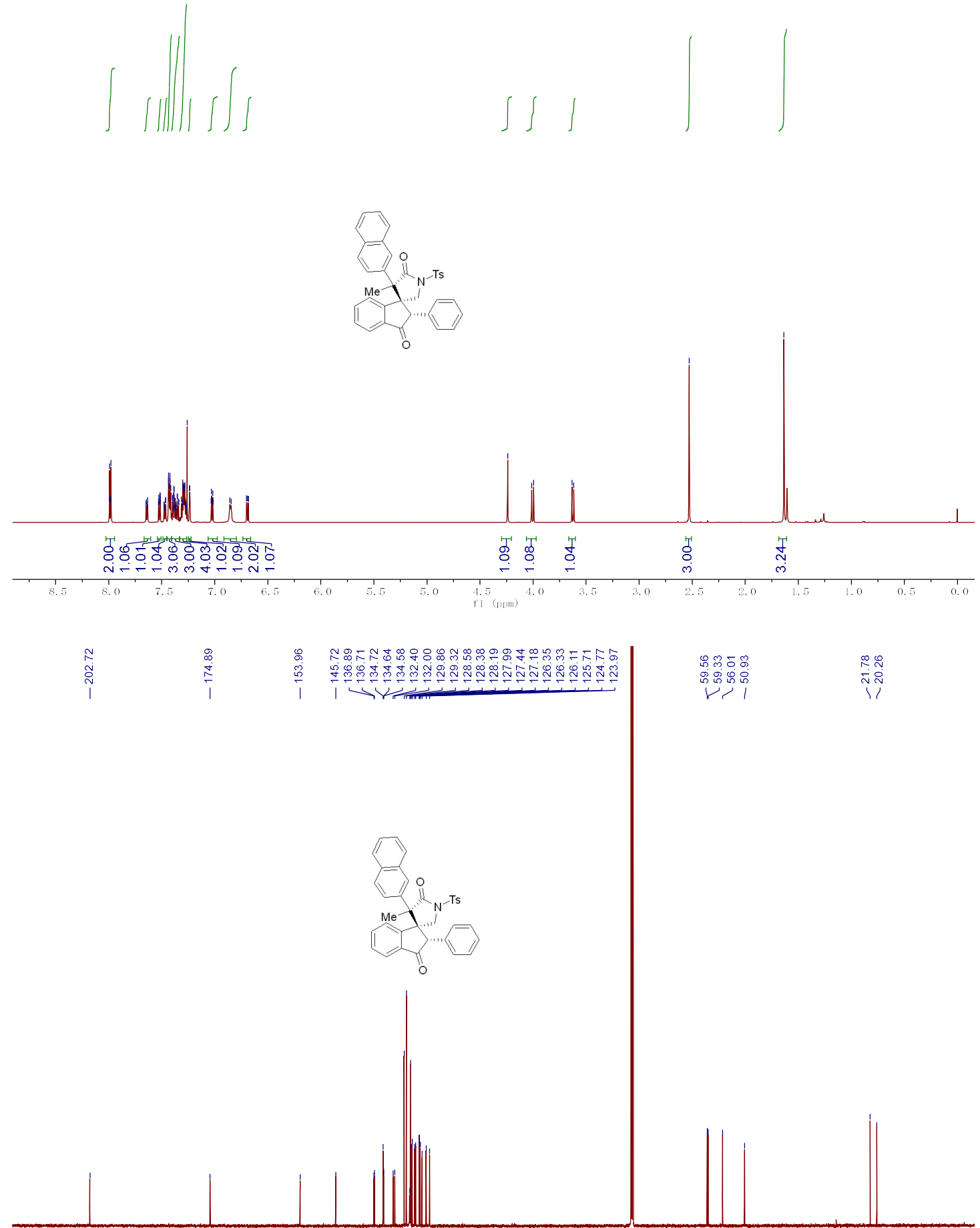

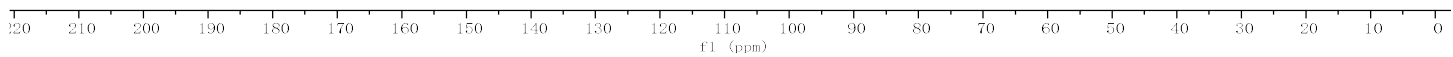




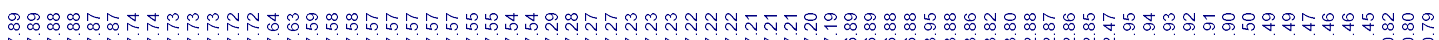

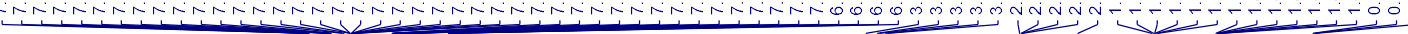
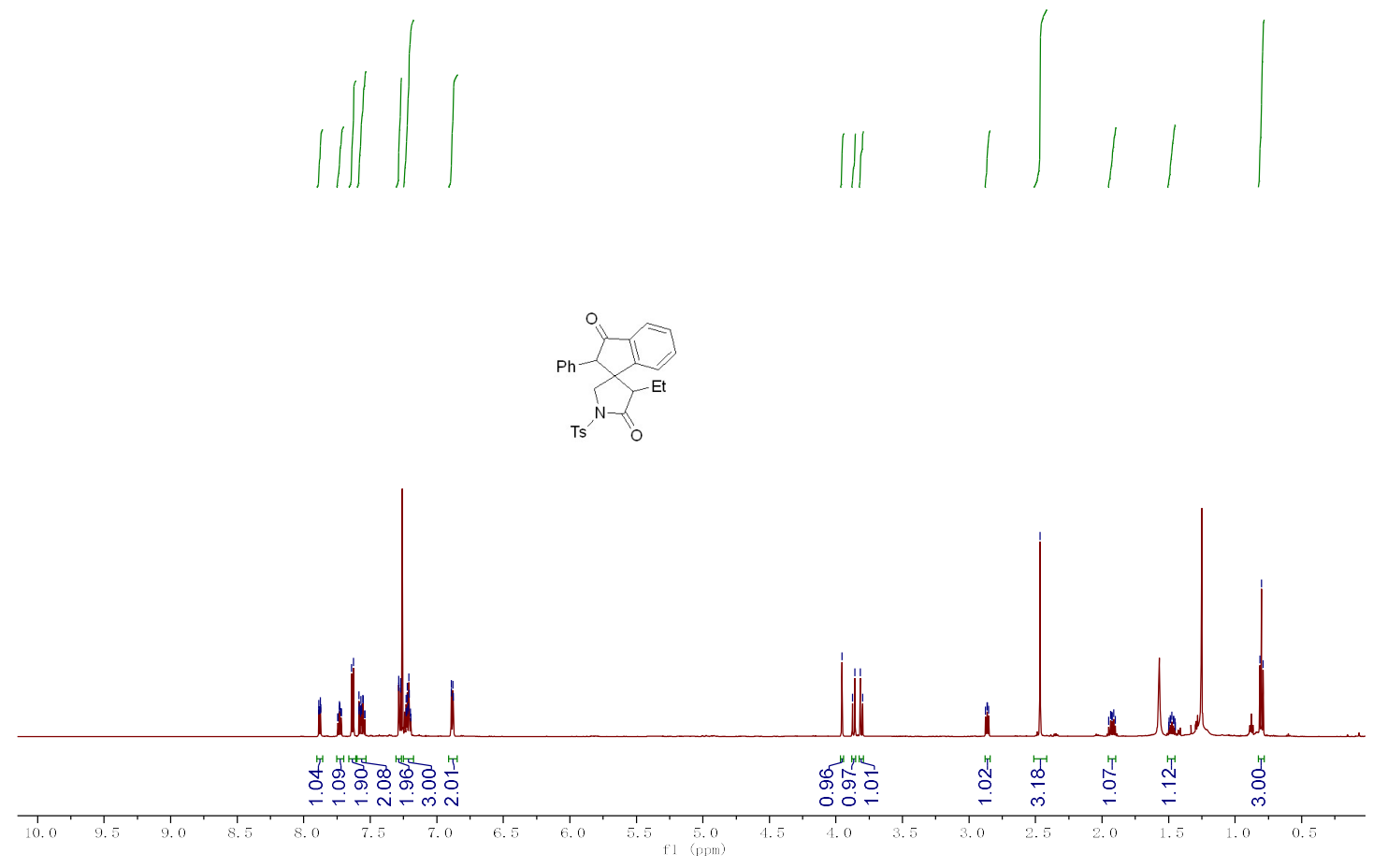

\begin{tabular}{|c|c|c|c|}
\hline 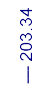 & 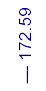 & 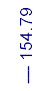 & 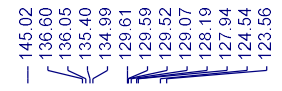 \\
\hline
\end{tabular}
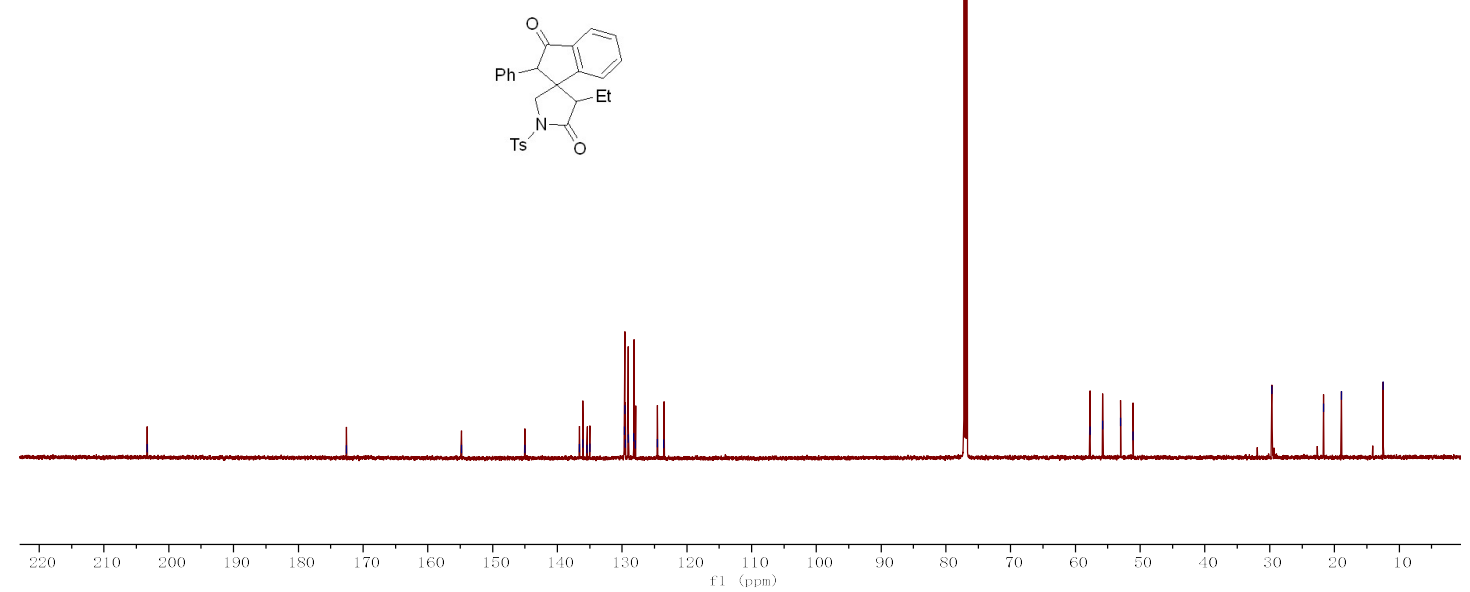
$3 \mathbf{z}$

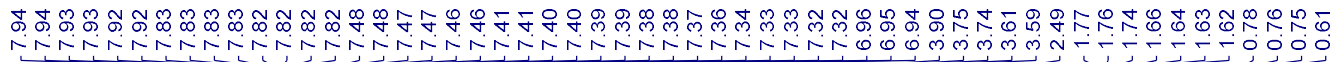
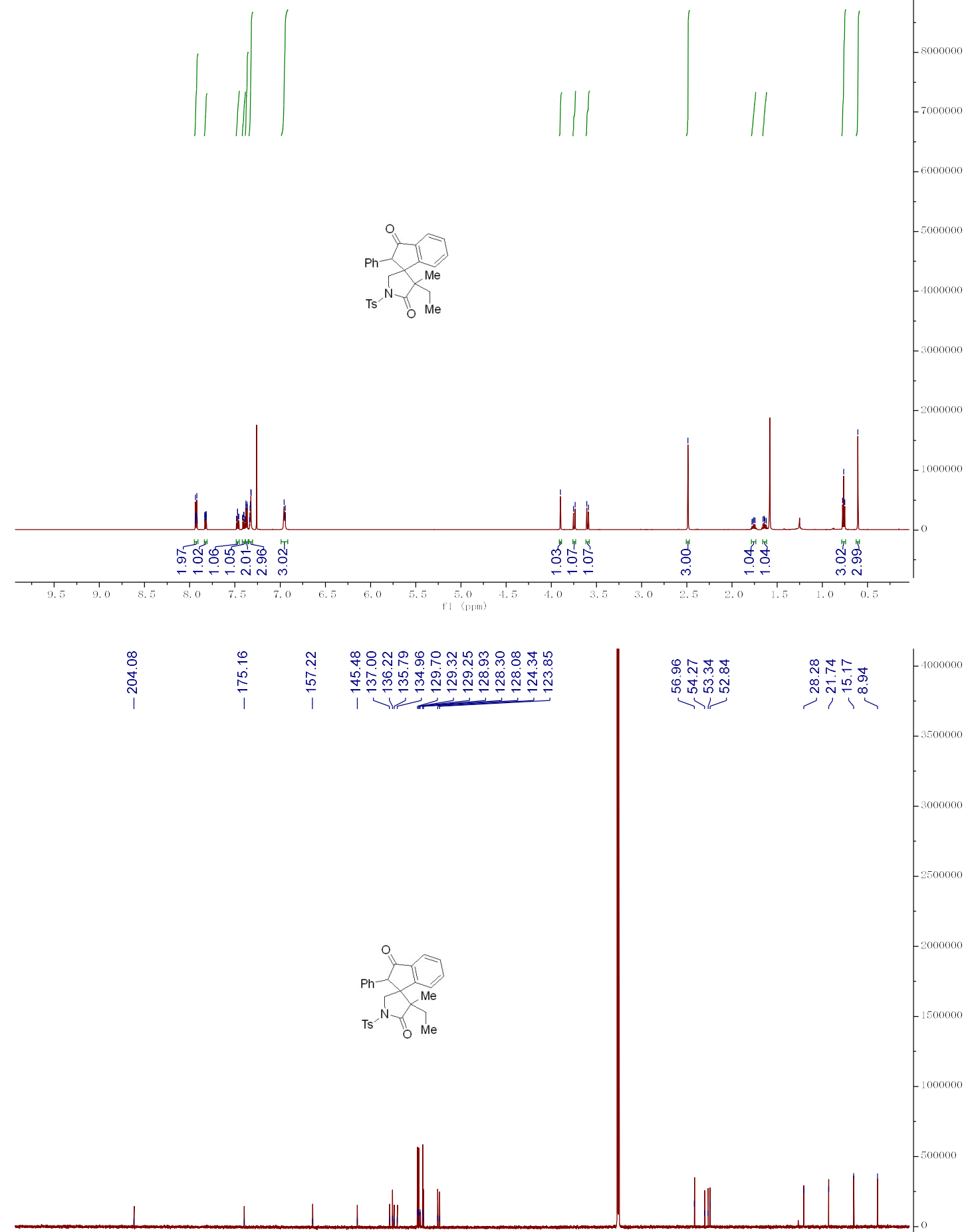

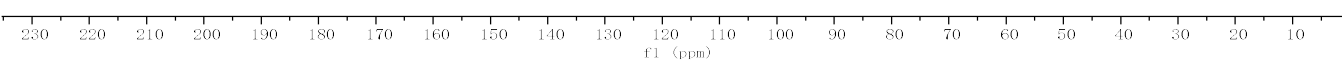


3aa

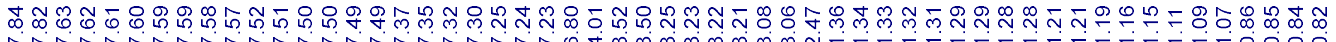

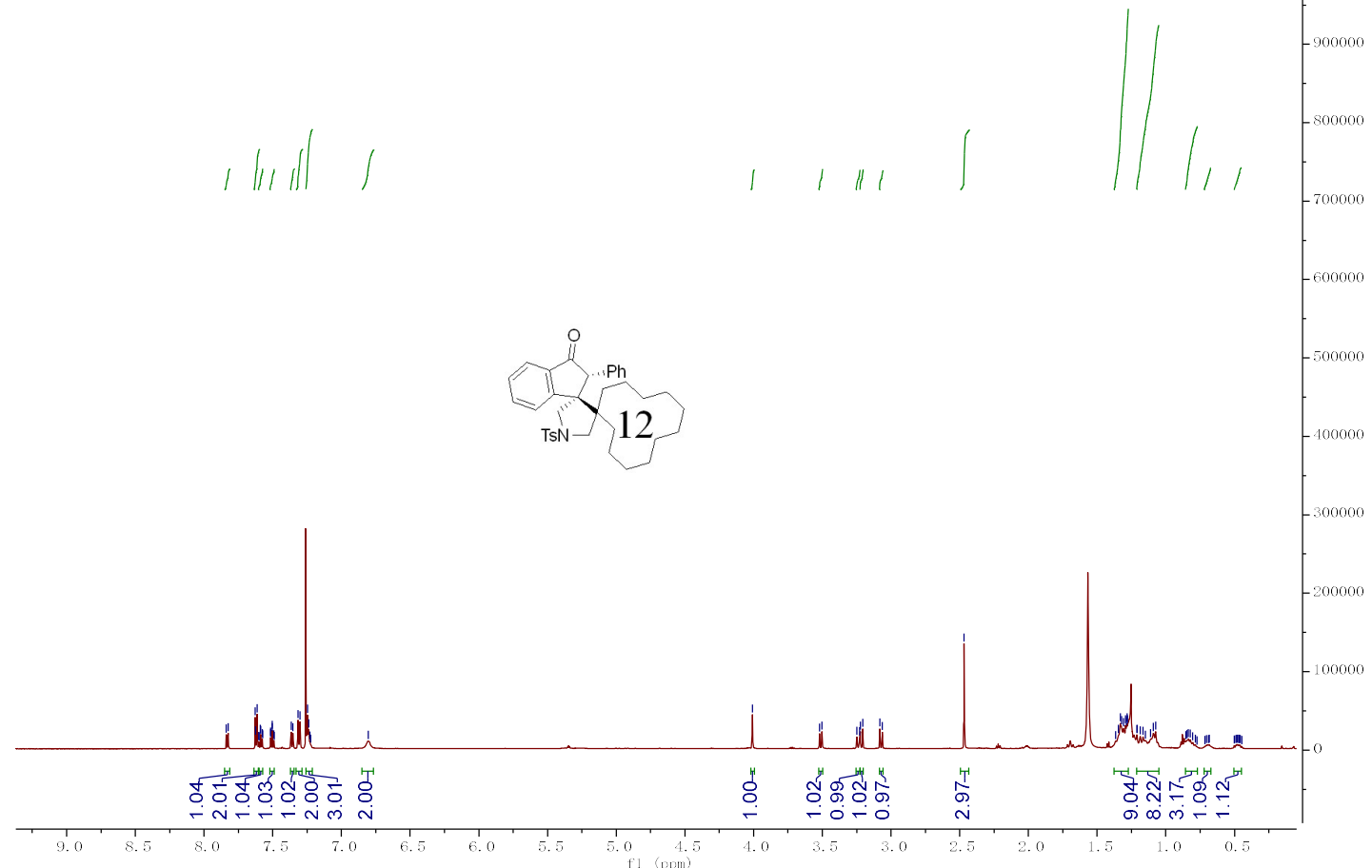

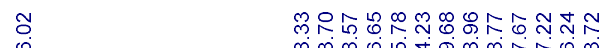

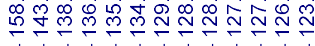

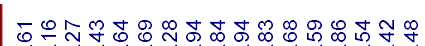

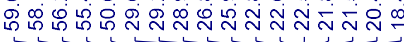
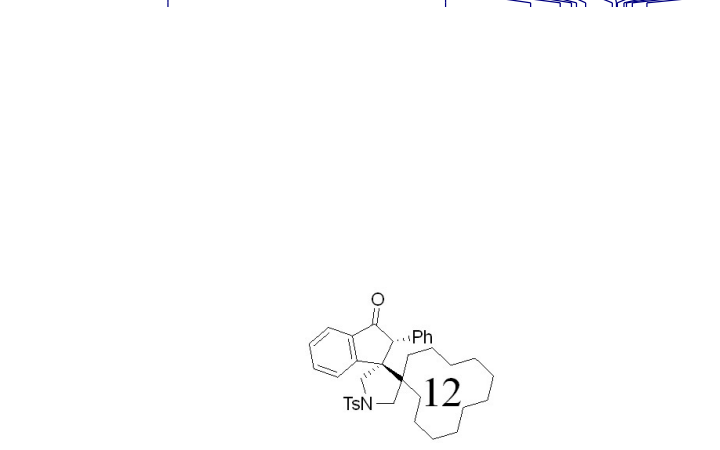

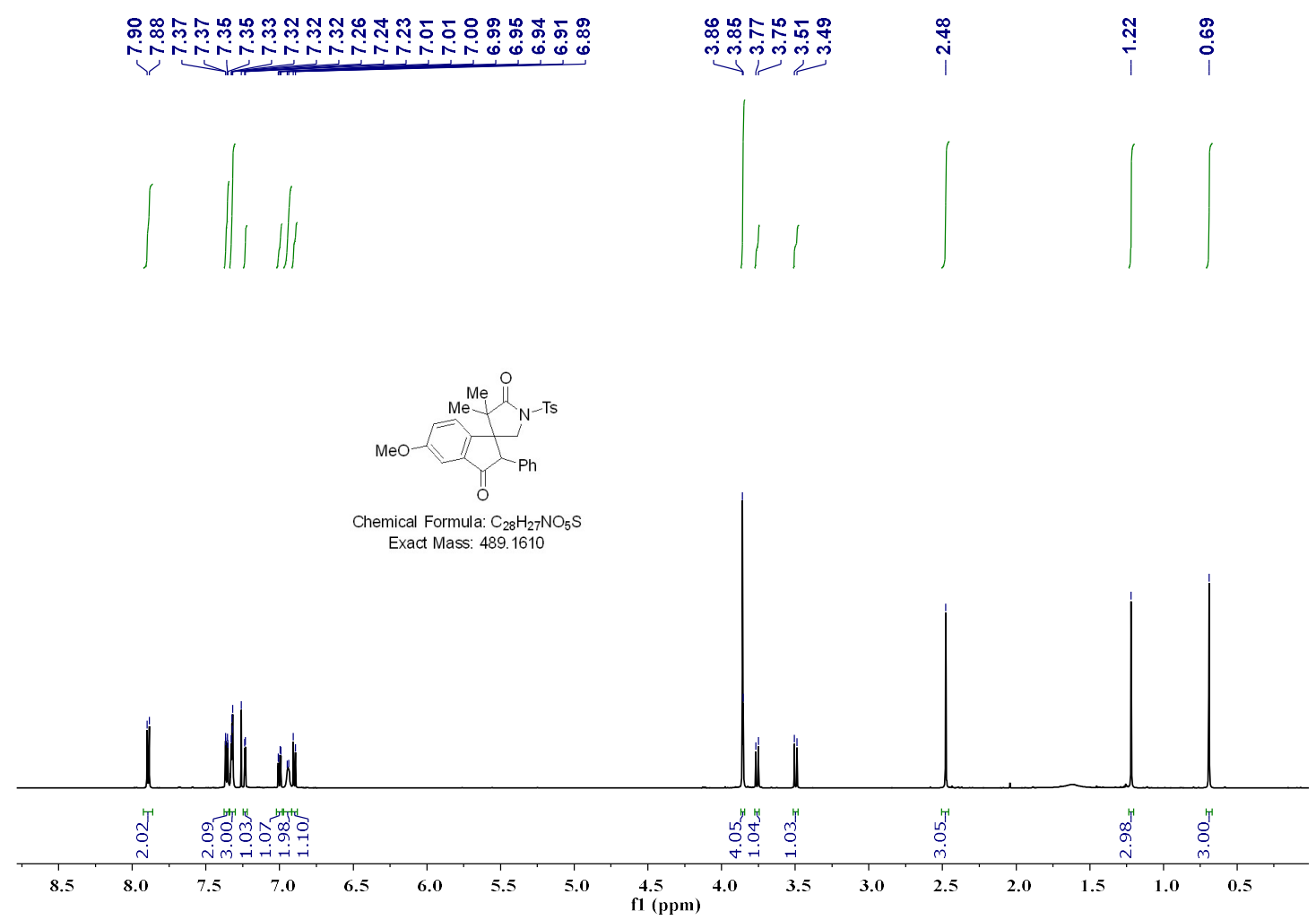

Chemical Formula: $\mathrm{C}_{28} \mathrm{H}_{27} \mathrm{NO}_{5} \mathrm{~S}$

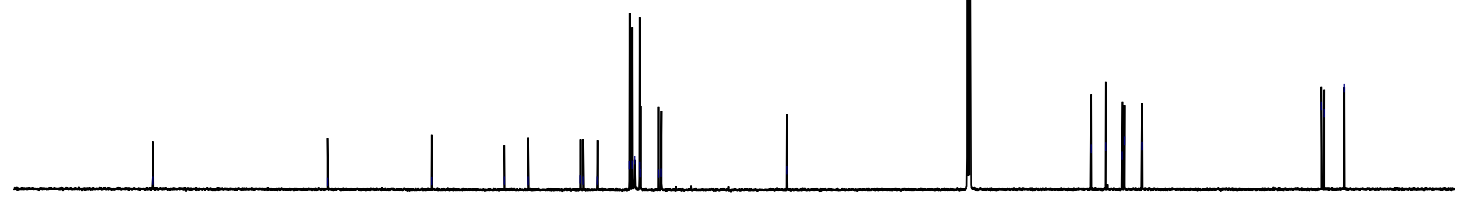

$\begin{array}{rlllllllllllllllllllll}220 & 210 & 200 & 190 & 180 & 170 & 160 & 150 & 140 & 130 & 120 & 110 & 100 & 90 & 80 & 70 & 60 & 50 & 40 & 30 & 20 & 10\end{array}$ 
$3 a c$

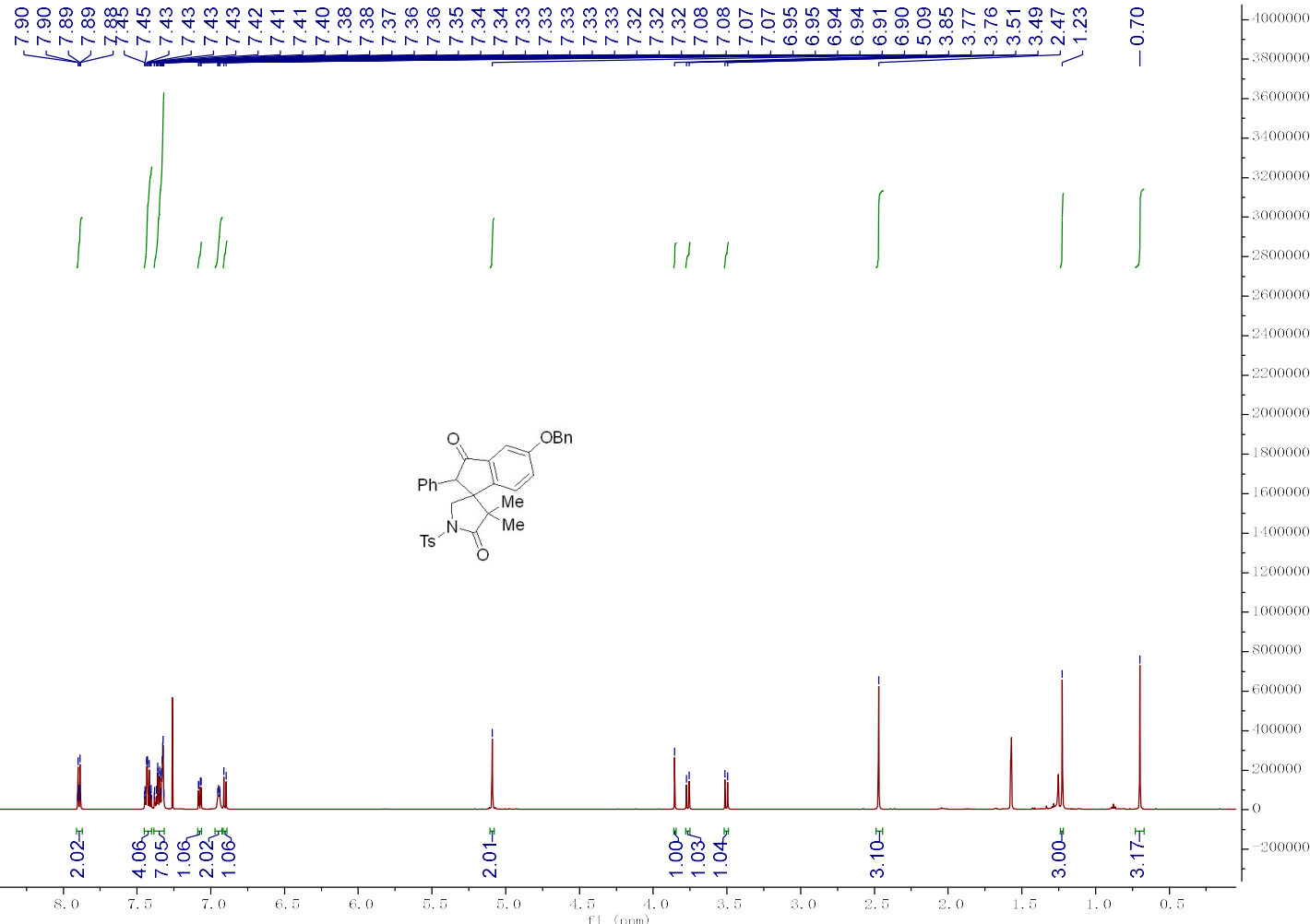

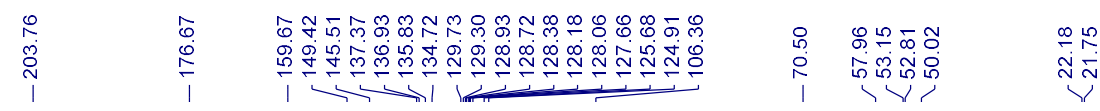

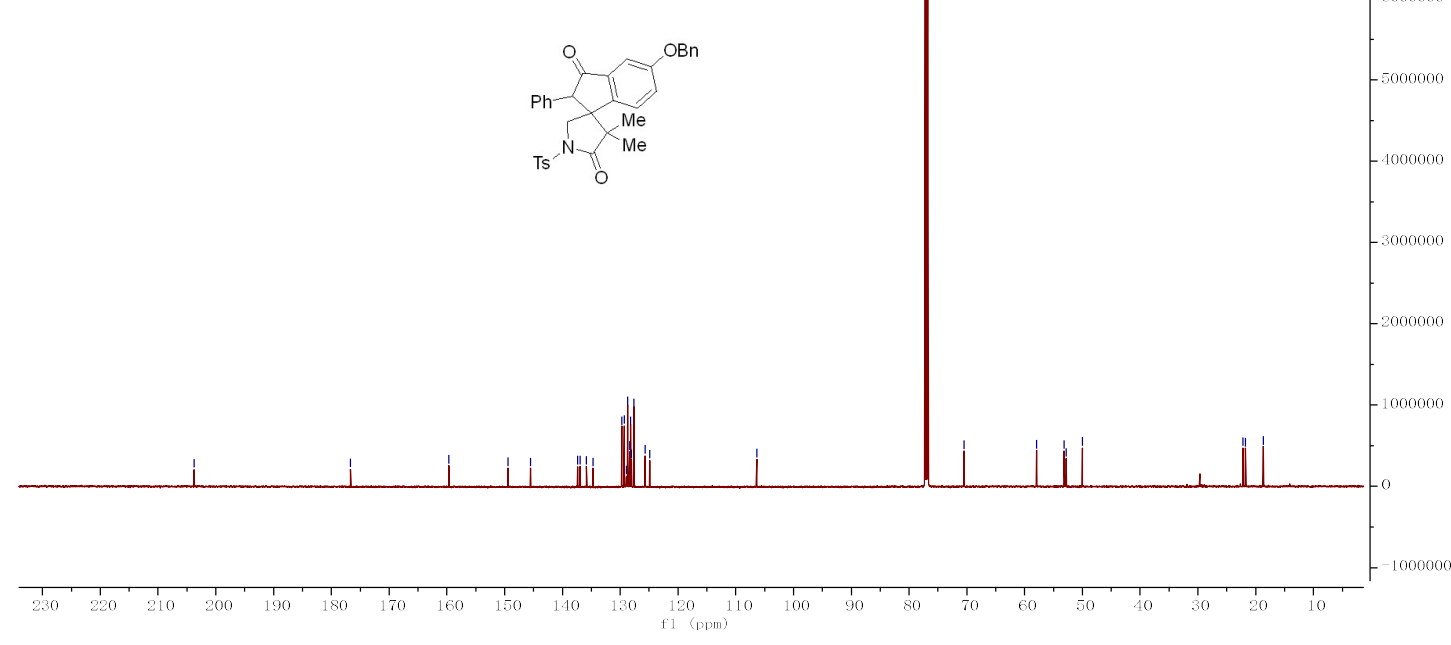



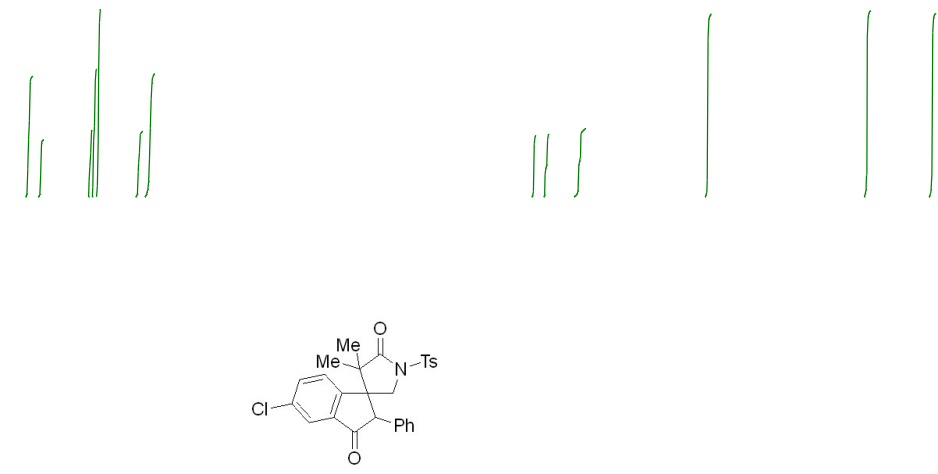

Chemical Formula: $\mathrm{C}_{27} \mathrm{H}_{24} \mathrm{ClNO}_{4}$

Exact Mass: 493.1115

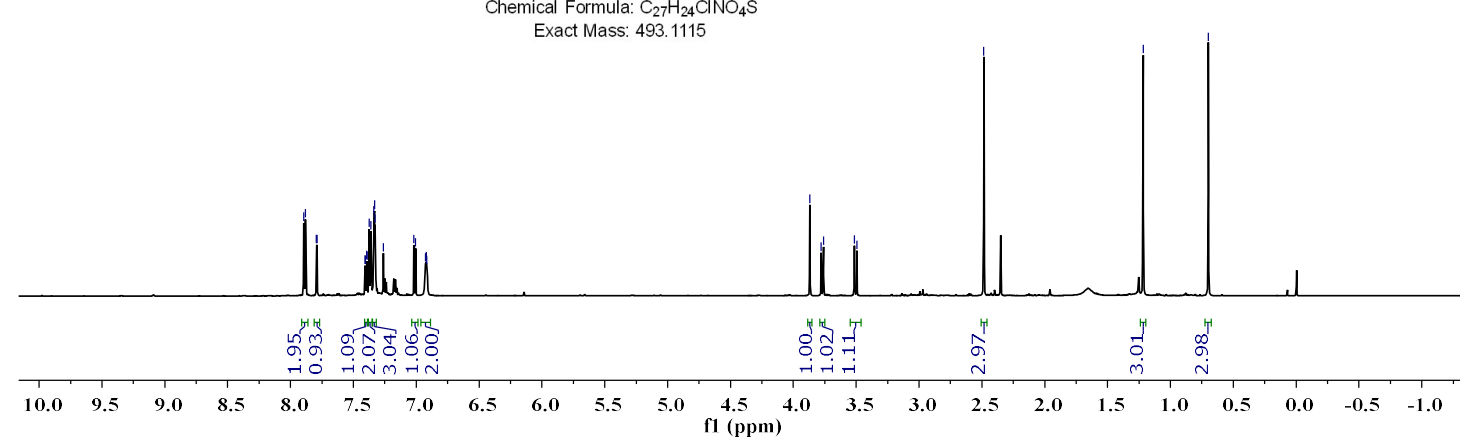

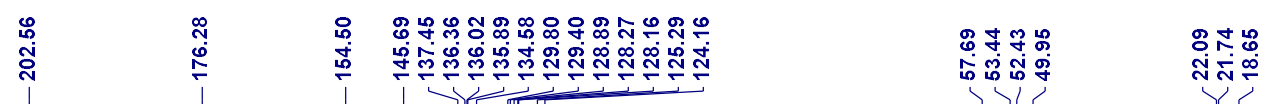

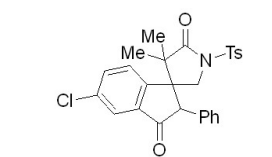

Chemical Formula: $\mathrm{C}_{27} \mathrm{H}_{24} \mathrm{ClNO}_{4} \mathrm{~S}$ Exact Mass: 493.1115

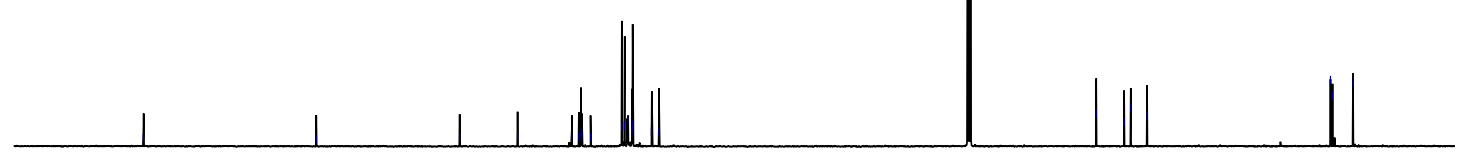

$\begin{array}{llllllllllllllllllllll}220 & 210 & 200 & 190 & 180 & 170 & 160 & 150 & 140 & 130 & 120 & 110 & 100 & 90 & 80 & 70 & 60 & 50 & 40 & 30 & 20 & 10\end{array}$ 
3ae

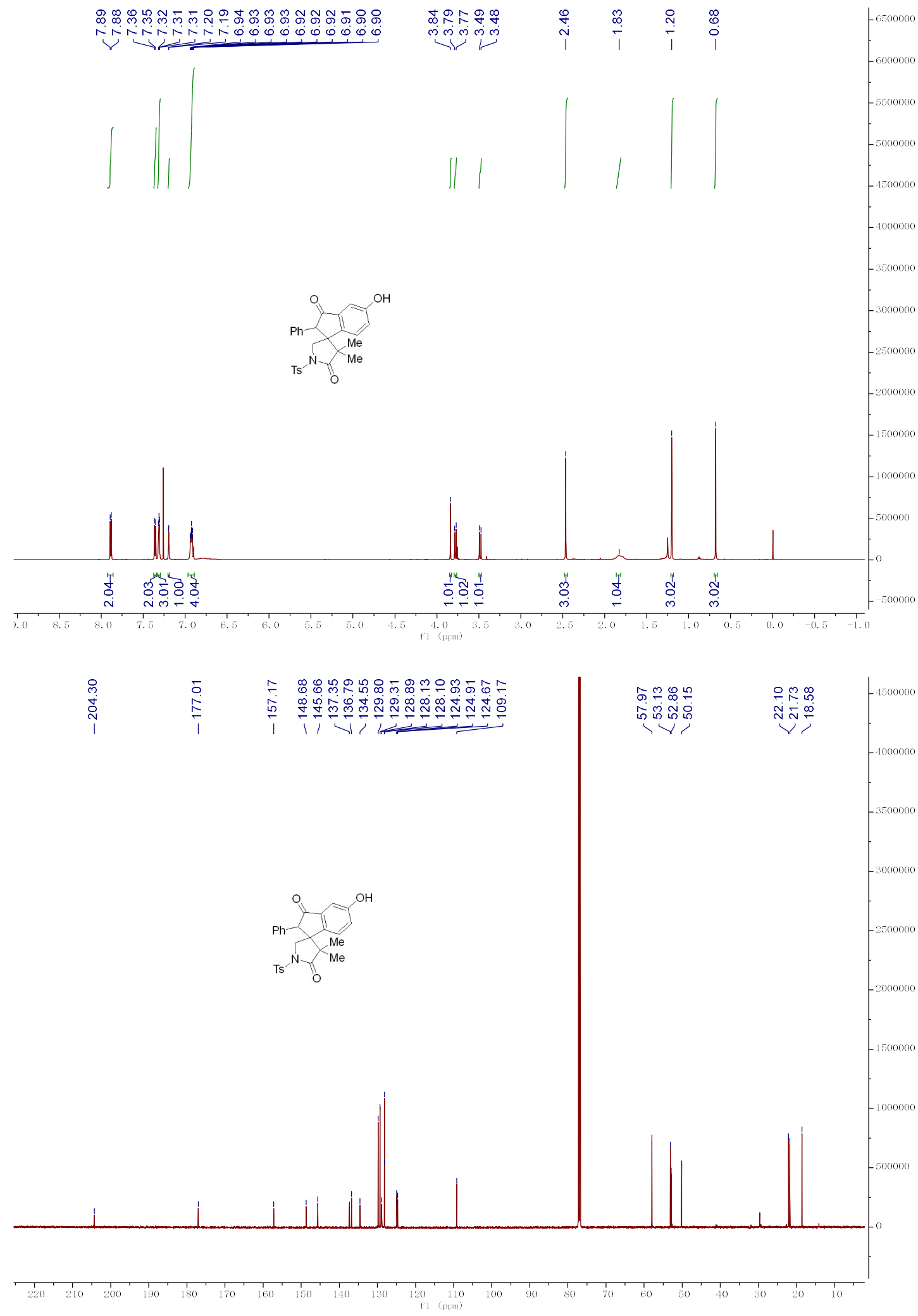




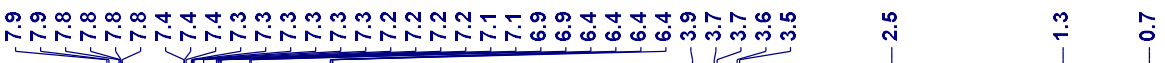
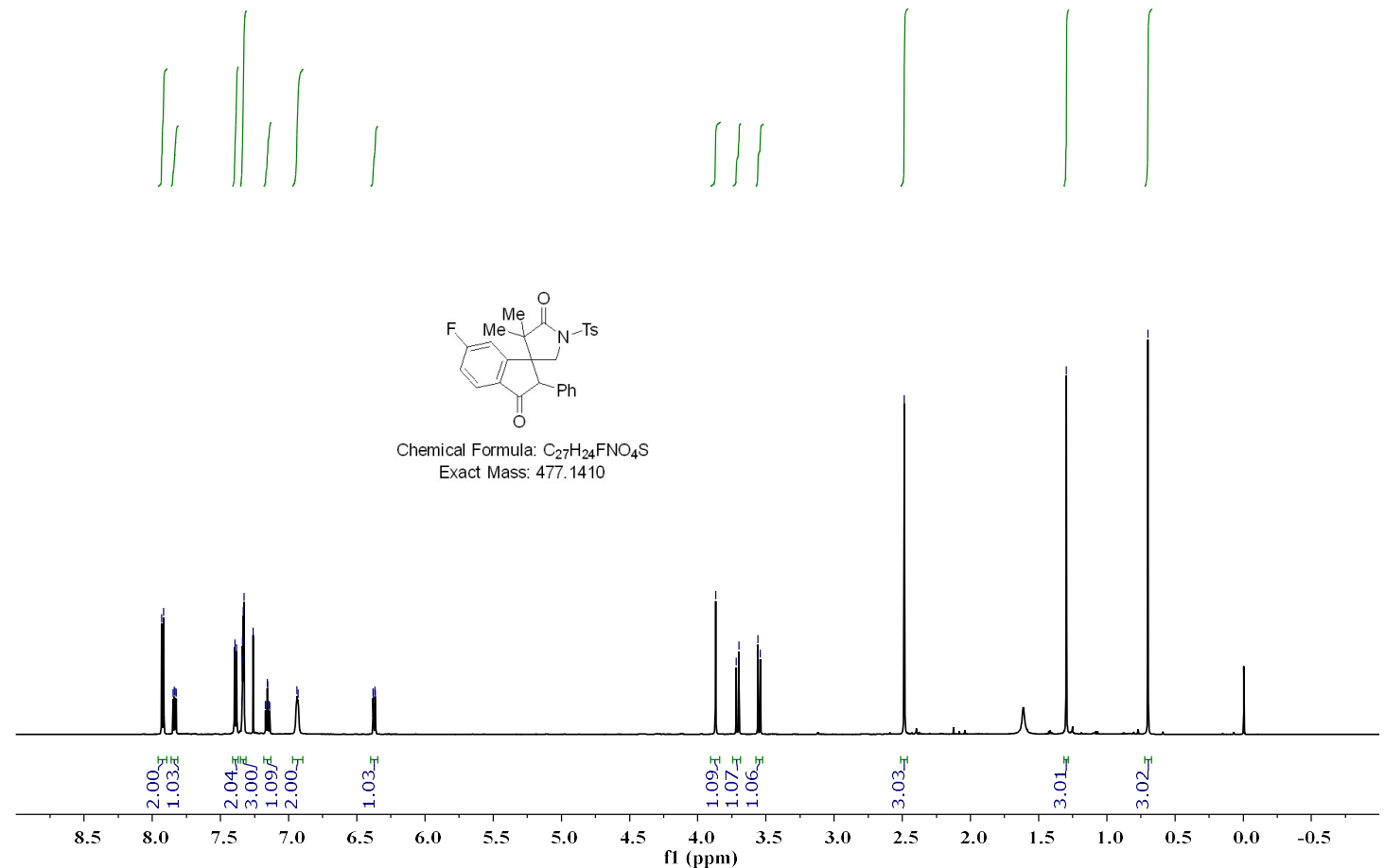

Chemical Formula: $\mathrm{C}_{27} \mathrm{H}_{24} \mathrm{FNO}_{4}$

Exact Mass: 477.1410
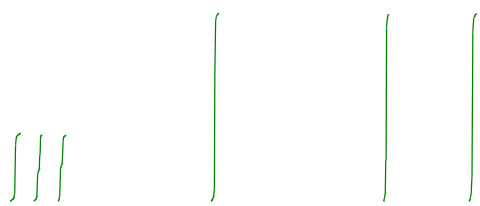

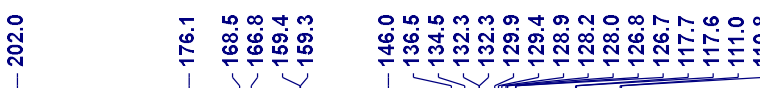

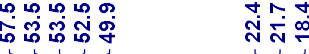

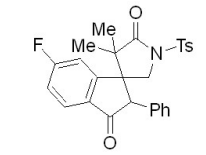

Chemical Formula: $\mathrm{C}_{27} \mathrm{H}_{24} \mathrm{FNO}_{4} \mathrm{~S}$

Exact Mass: 477.1410

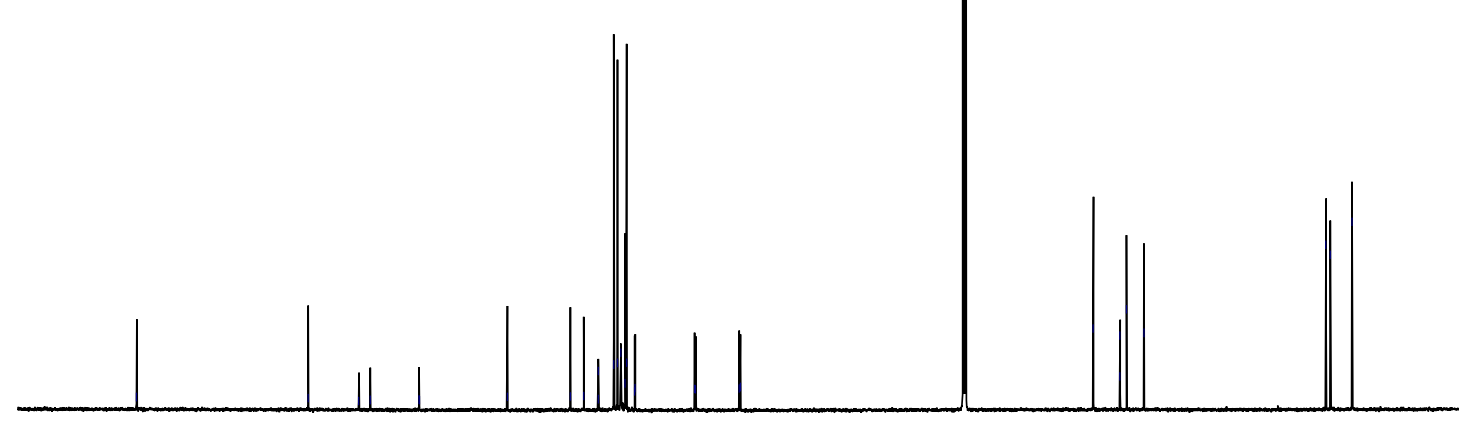

$\begin{array}{lllllllllllllllllllll}210 & 200 & 190 & 180 & 170 & 160 & 150 & 140 & 130 & 120 & 110 & 100 & 90 & 80 & 70 & 60 & 50 & 40 & 30 & 20 & 10\end{array}$ 


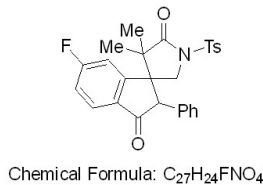

mo

Comical Formula. $\mathrm{C}_{27} \mathrm{H}_{24} \mathrm{FNO}_{4} \mathrm{~S}$

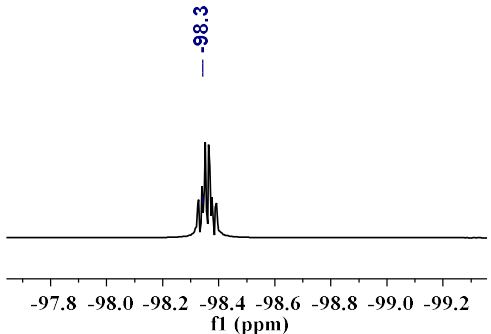

f1 (ppm)

$\begin{array}{lllllllllllllllllllll}100 & 80 & 60 & 40 & 20 & 0 & -20 & -40 & -60 & -80 & -100 & -120 & -140 & -160 & -180 & -200 & -220 & -240 & -260 & -280 & -300\end{array}$ 
3ag

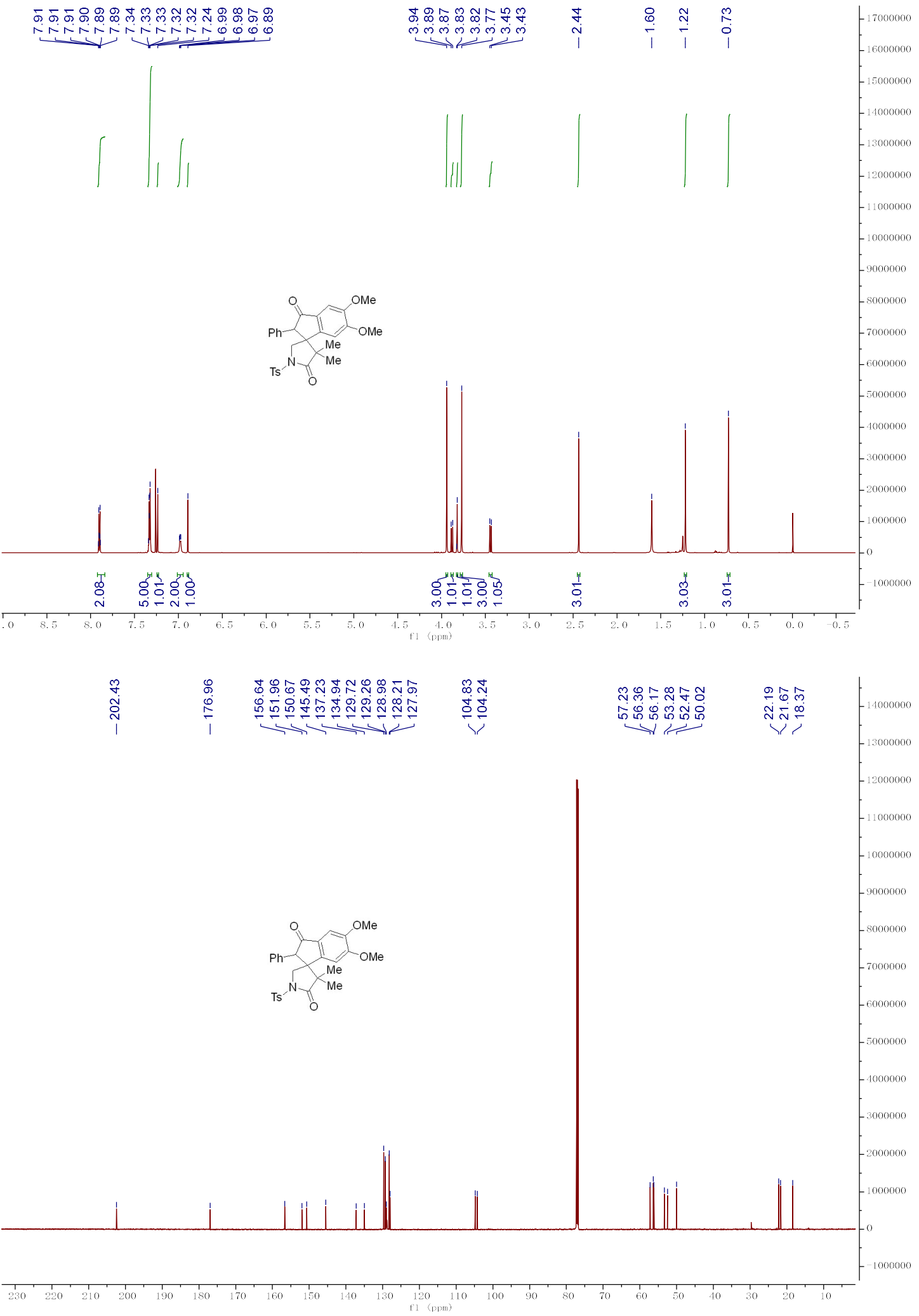


3ah

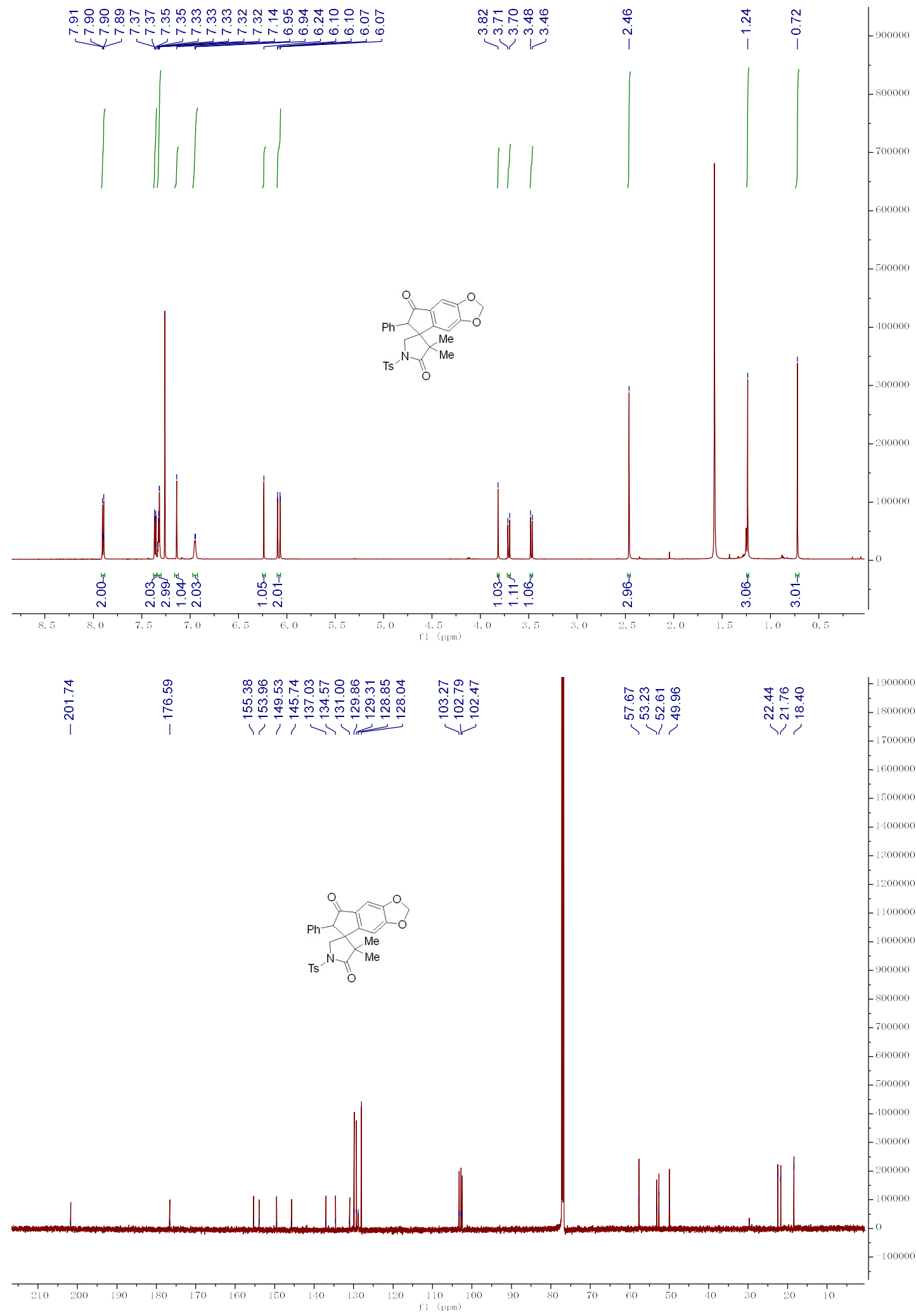


3ai
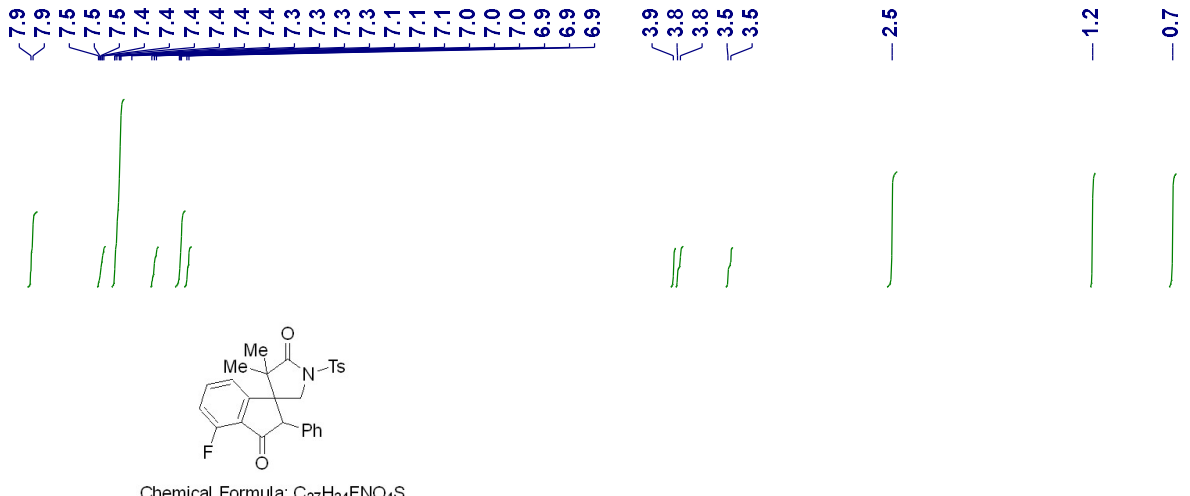

Chemical Formula: $\mathrm{C}_{27} \mathrm{H}_{24} \mathrm{FNO}_{4}$

Exact Mass: 477.1410

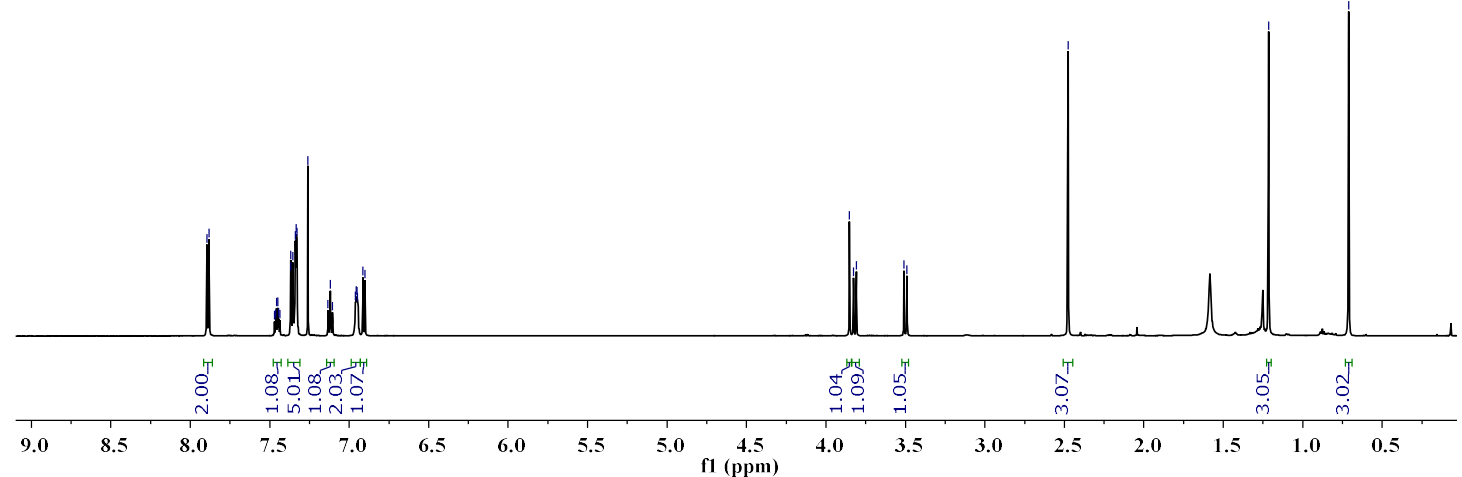

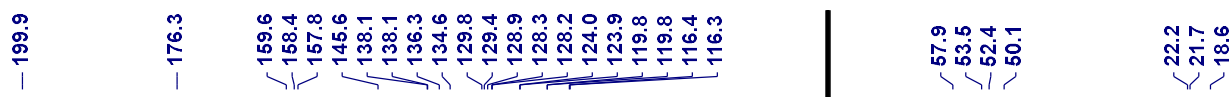

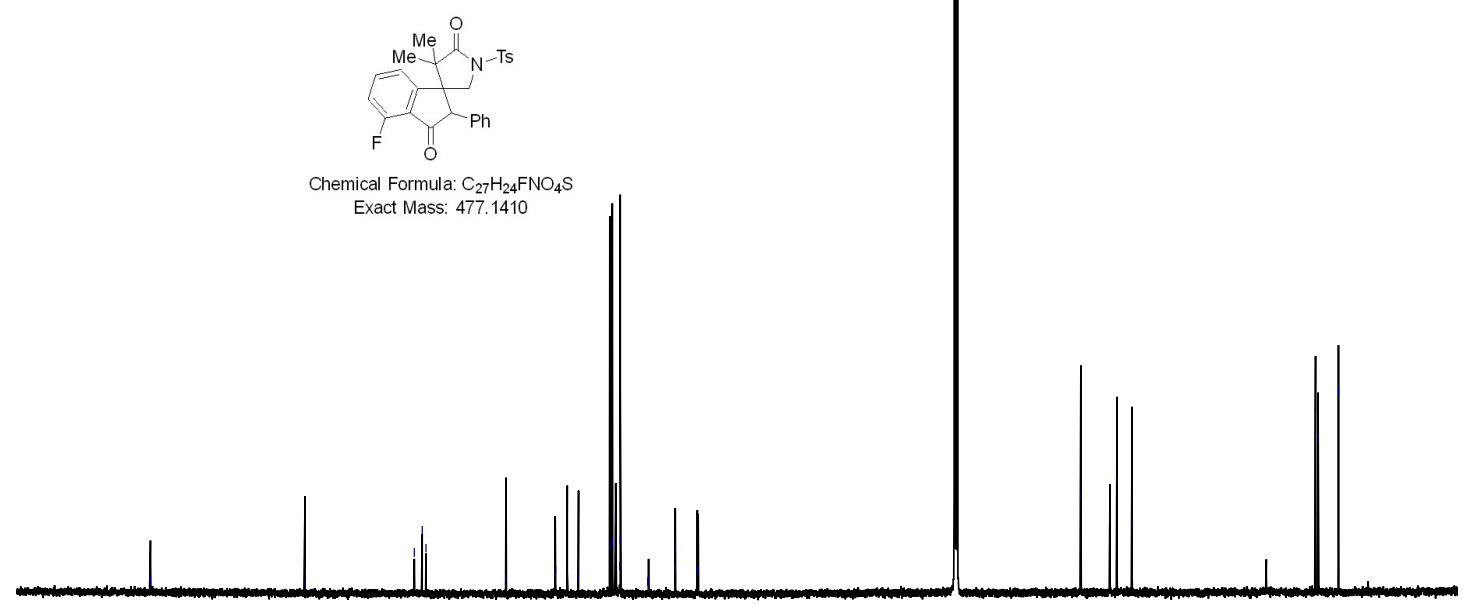

\begin{tabular}{lllllllllllllllllllllllllll}
\hline 20 & 210 & 200 & 190 & 180 & 170 & 160 & 150 & 140 & 130 & 120 & 110 & 100 & 90 & 80 & 70 & 60 & 50 & 40 & 30 & 20 & 10
\end{tabular} 


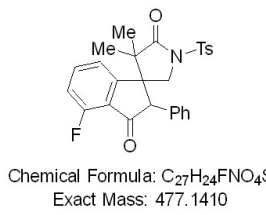

$\stackrel{\infty}{\stackrel{\infty}{i}}$
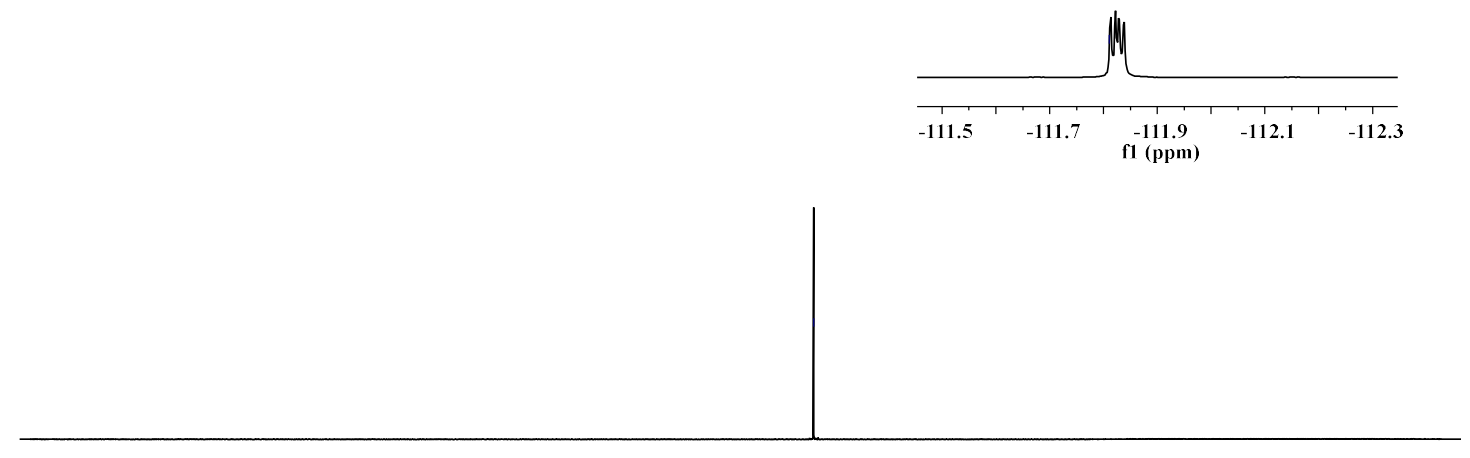

$\begin{array}{llllllllllllllllllllllll}10 & 0 & -10 & -20 & -30 & -40 & -50 & -60 & -70 & -80 & -90 & -100 & -110 & -120 & -130 & -140 & -150 & -160 & -170 & -180 & -190 & -200 & -210\end{array}$ 
3aj

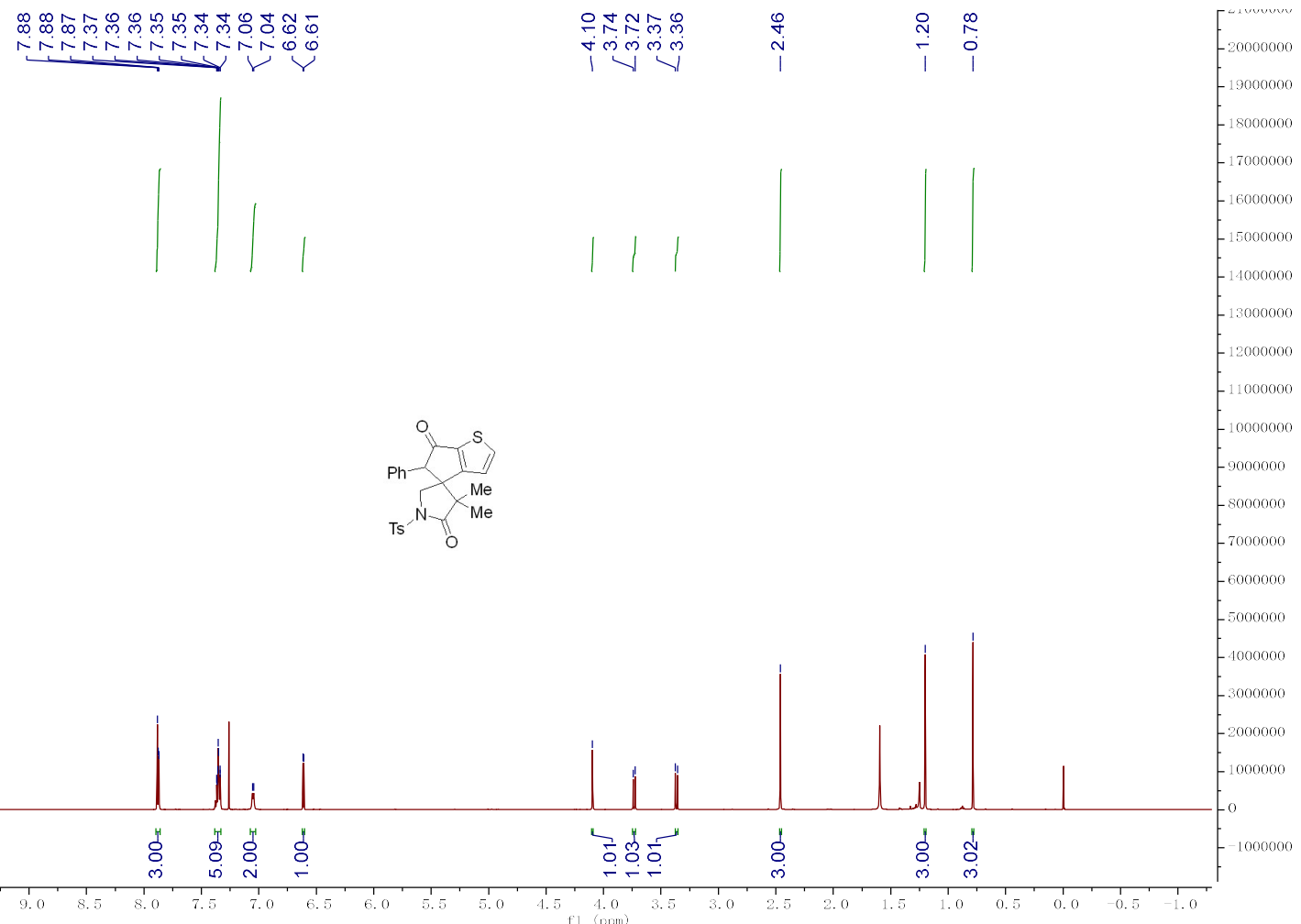

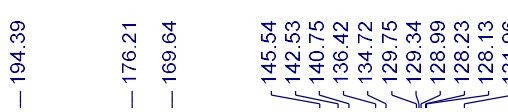

䑻 太令

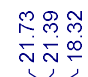

15000000

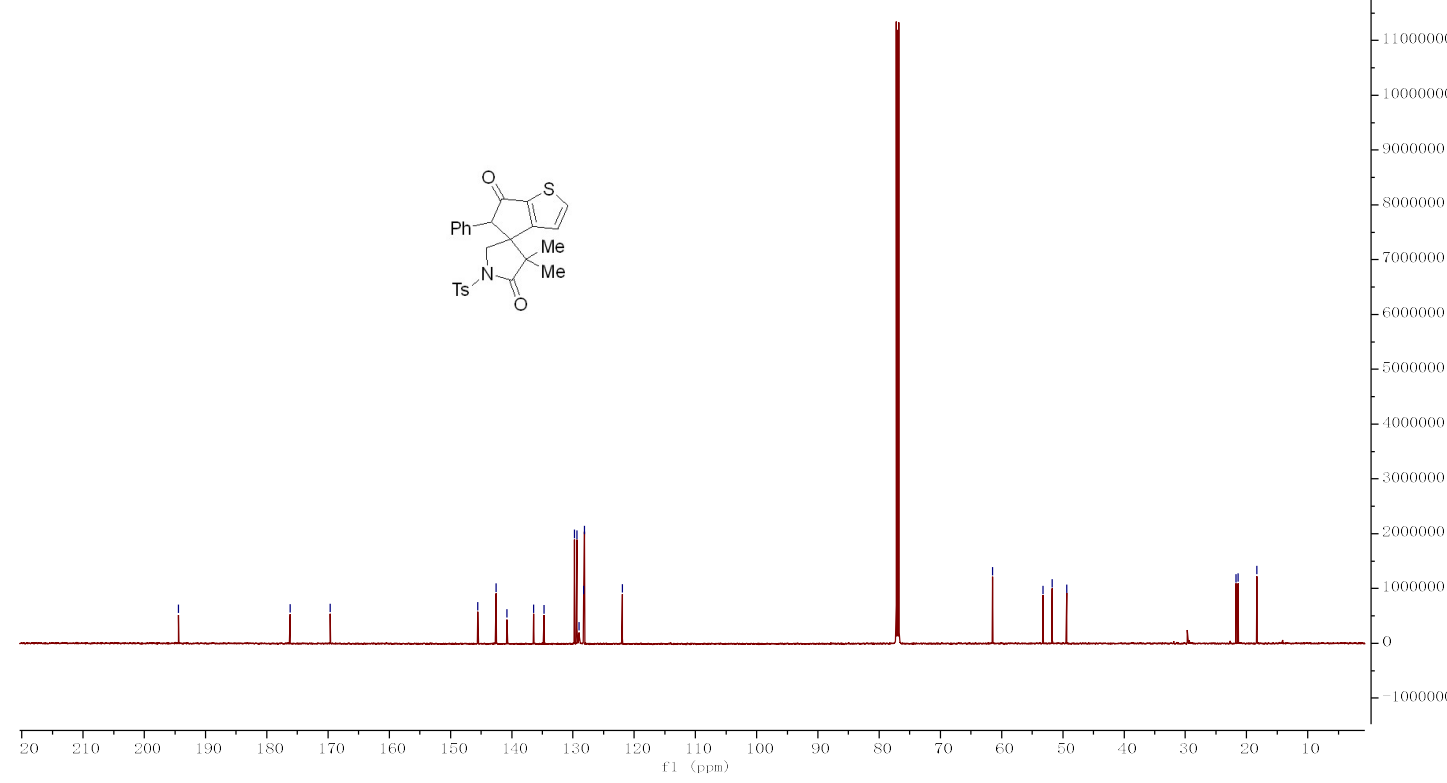



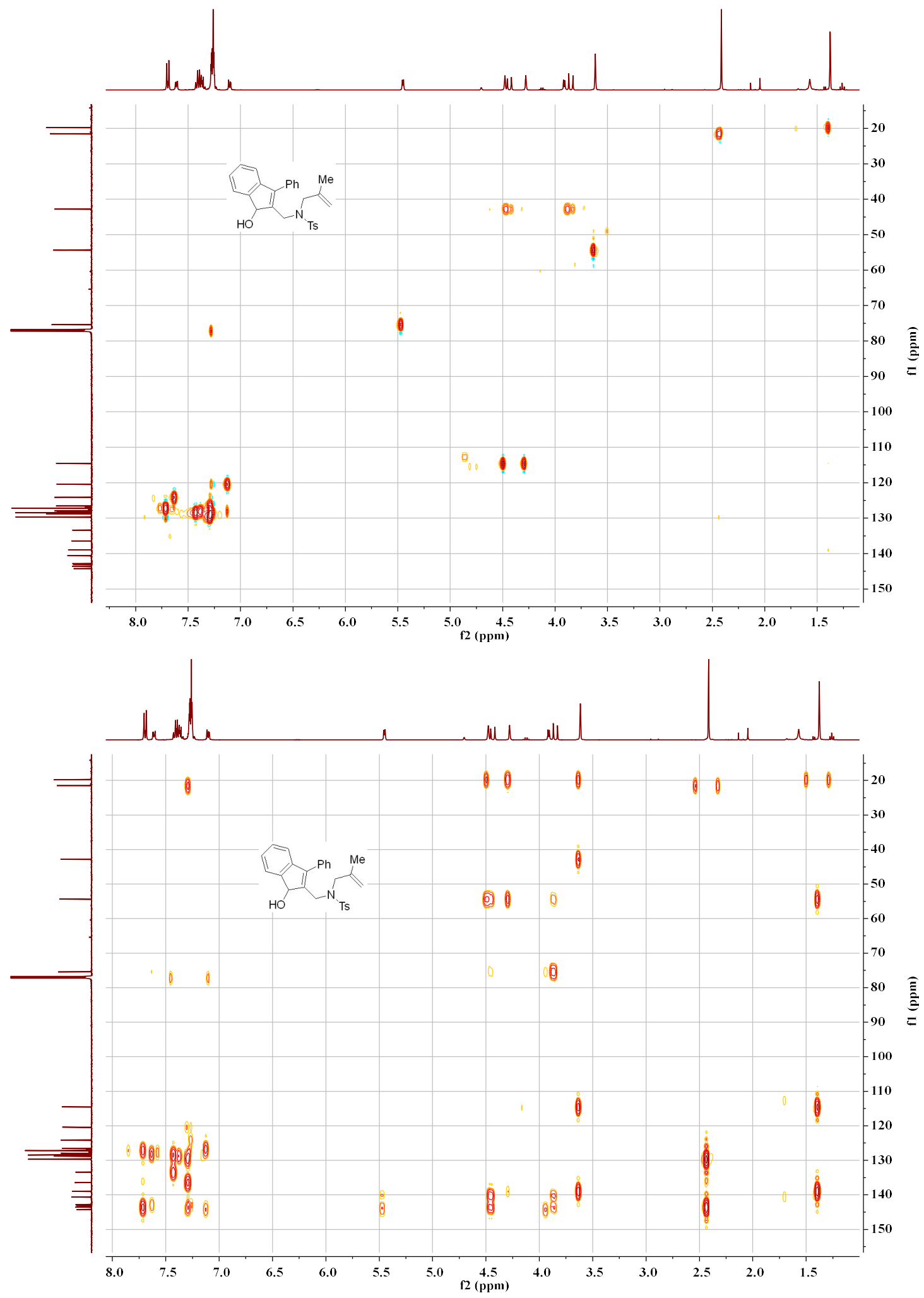


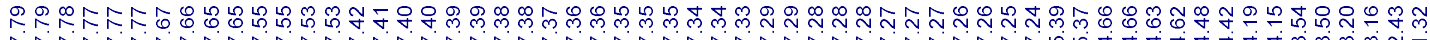

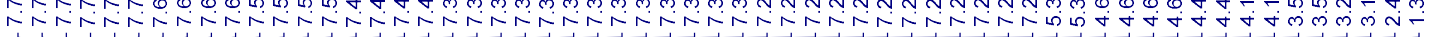
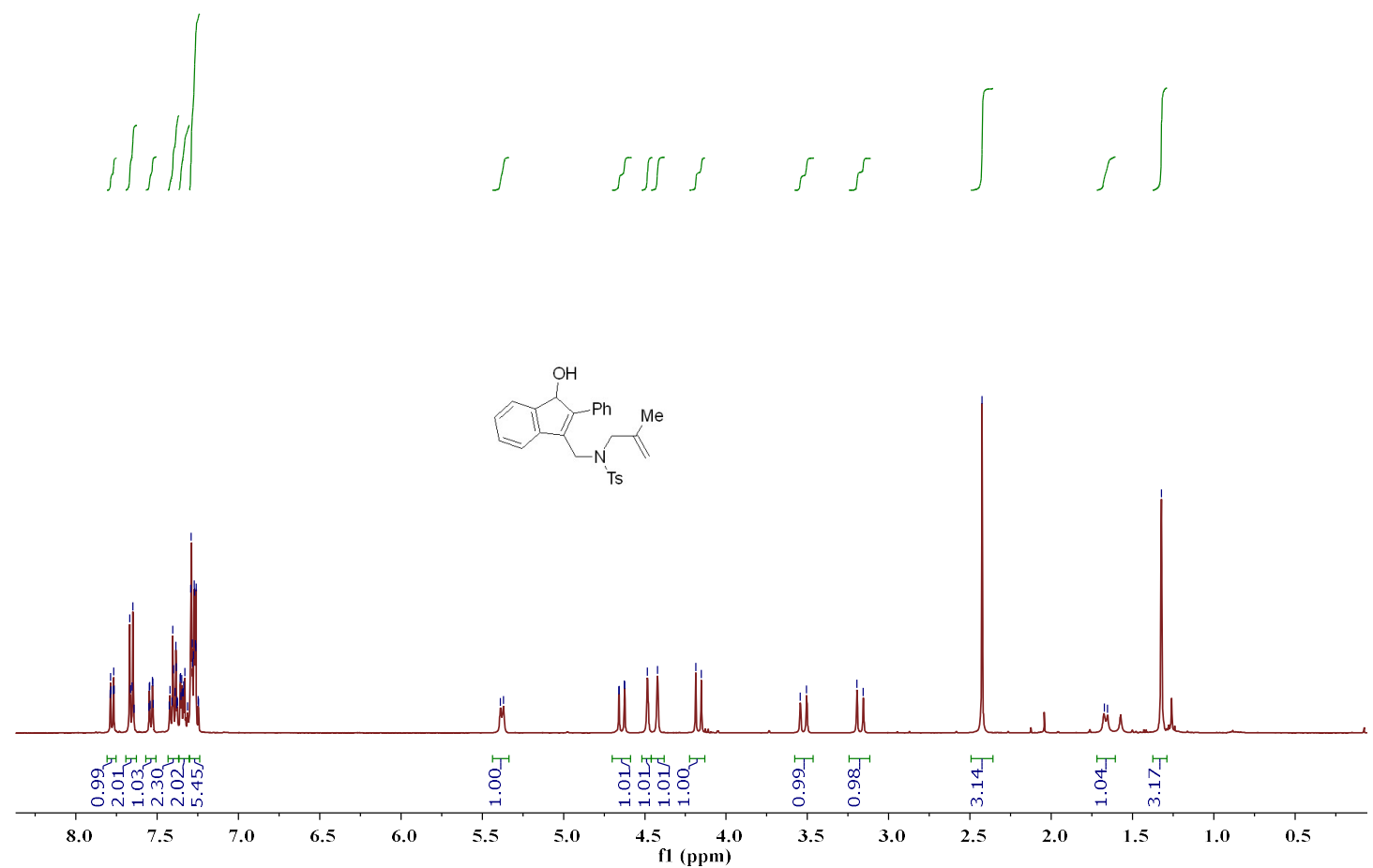

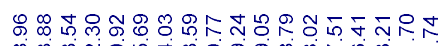

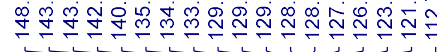
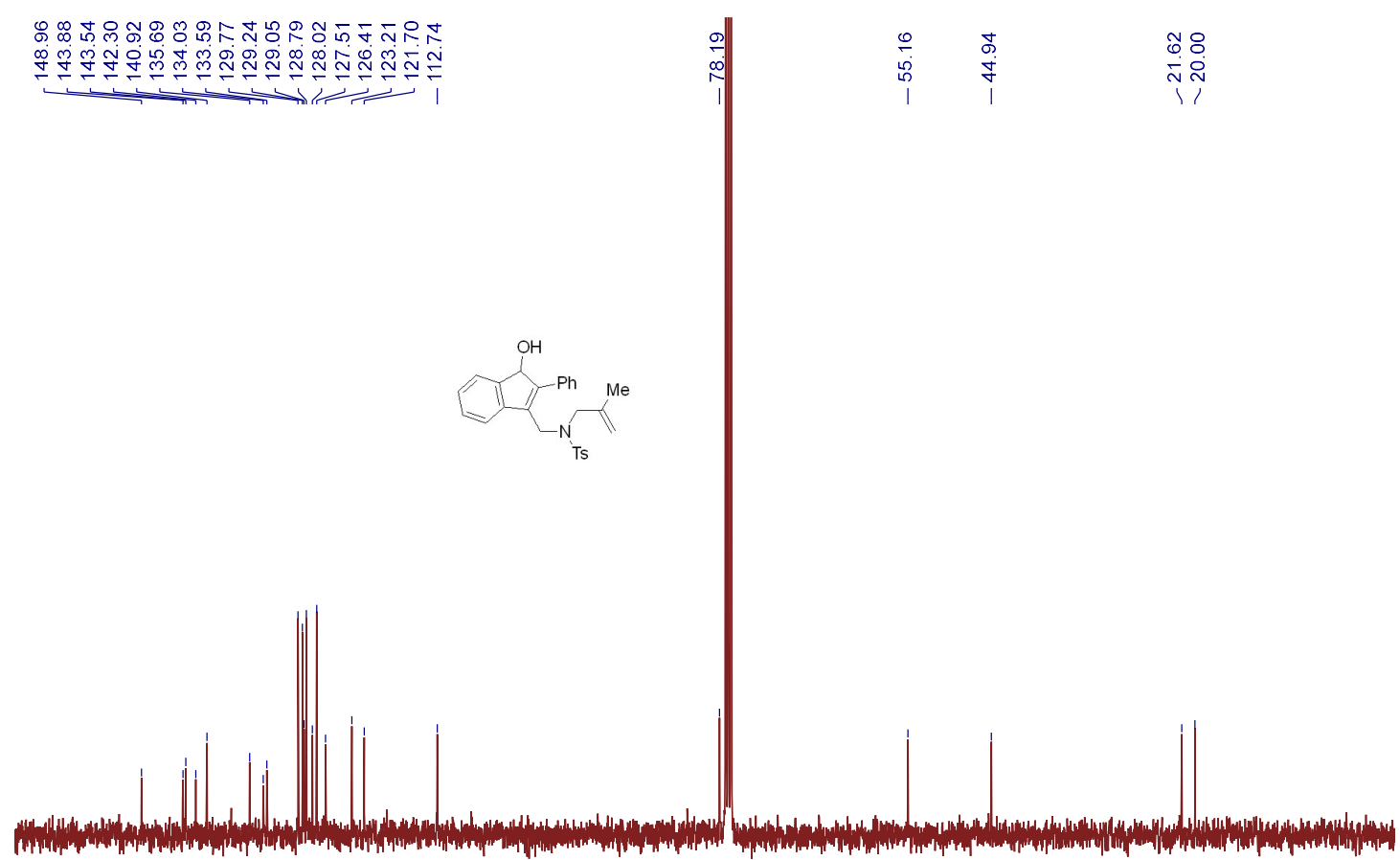

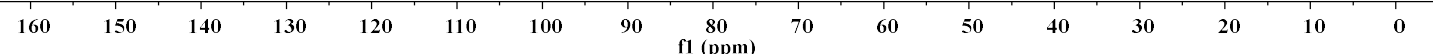


$4 m^{\prime}$

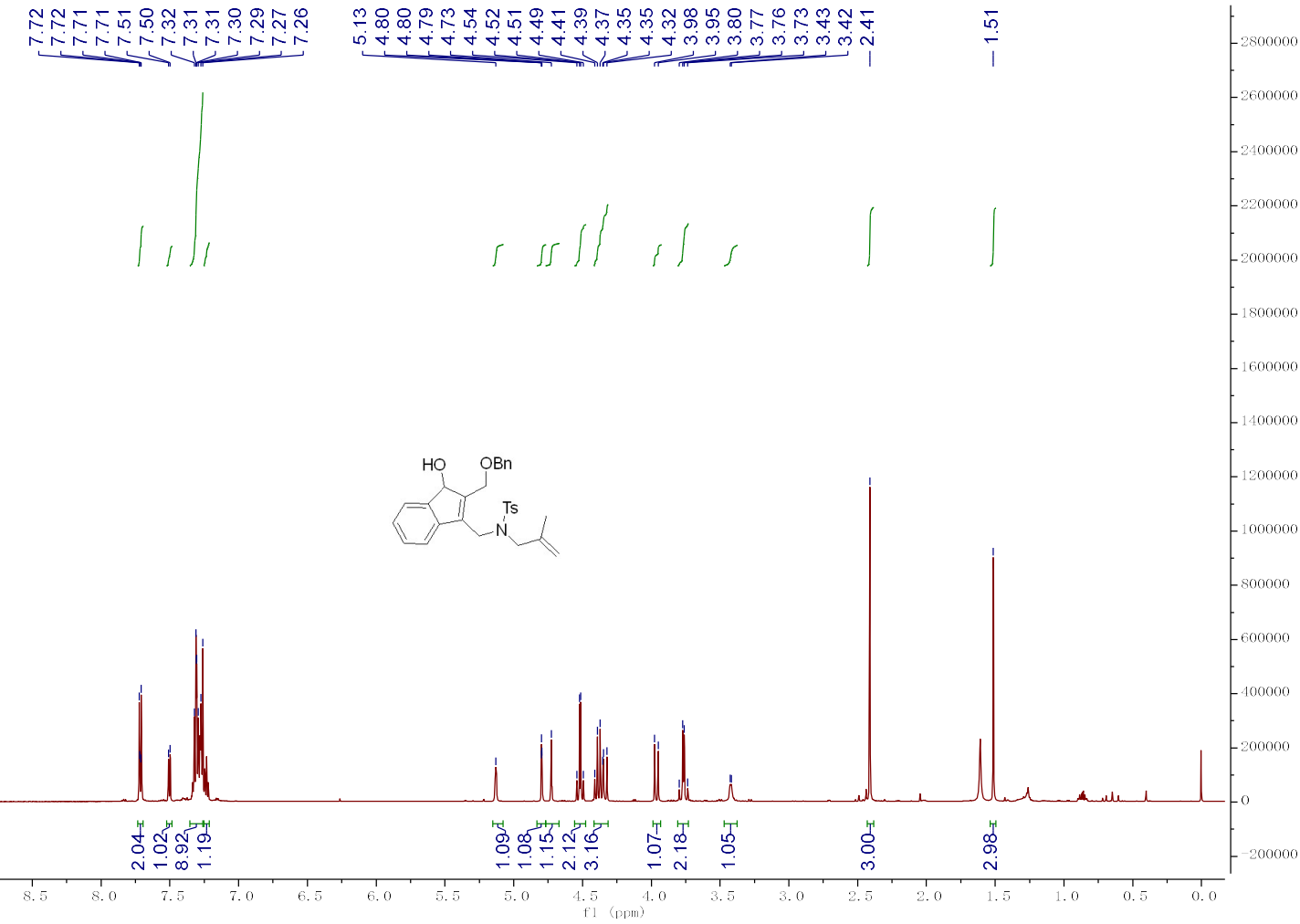

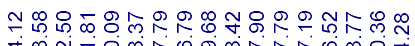

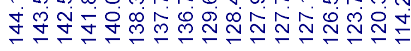

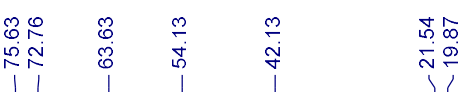

\begin{tabular}{l}
$\mid-8500000$ \\
-8000000 \\
-7500000 \\
-7000000 \\
\hline-6500000 \\
\hline-6000000 \\
\hline-5500000 \\
\hline-5000000 \\
\hline-4500000 \\
\hline-4000000 \\
\hline-3500000 \\
\hline-3000000 \\
\hline-2500000 \\
\hline-8000000 \\
\hline-1500000 \\
\hline-1000000 \\
\hline-500000 \\
\hline-0 \\
\hline-500000 \\
\hline
\end{tabular} 

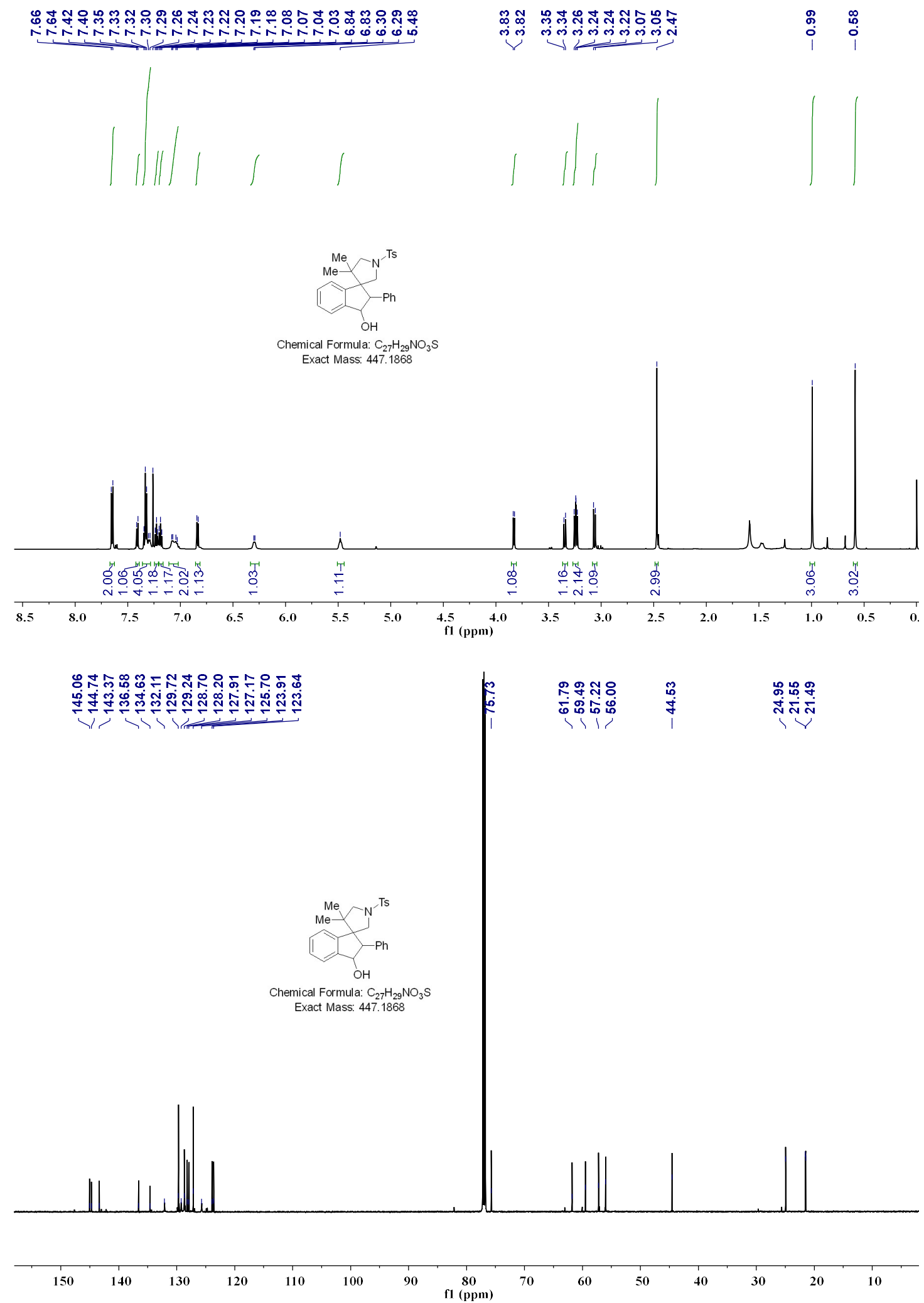

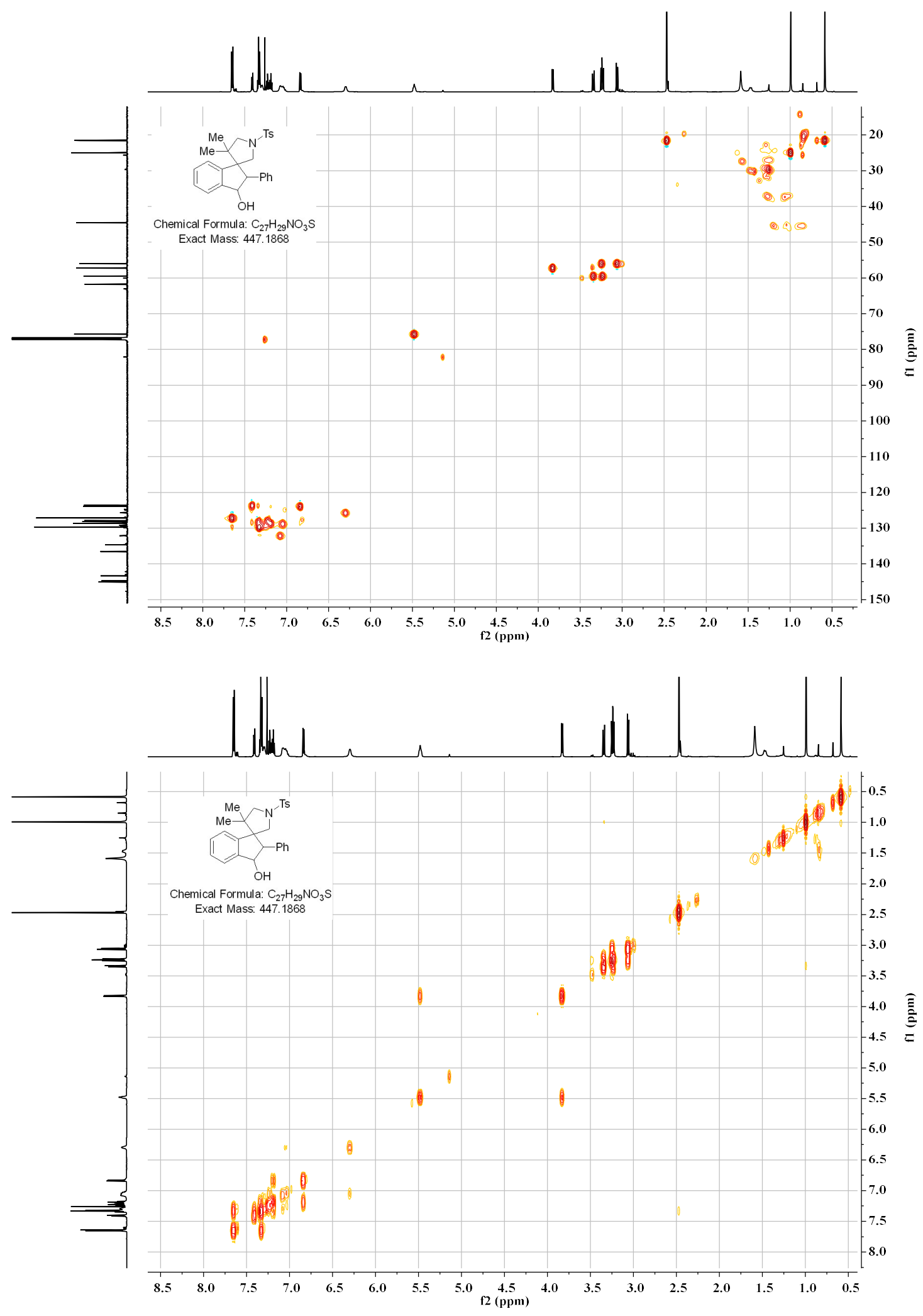


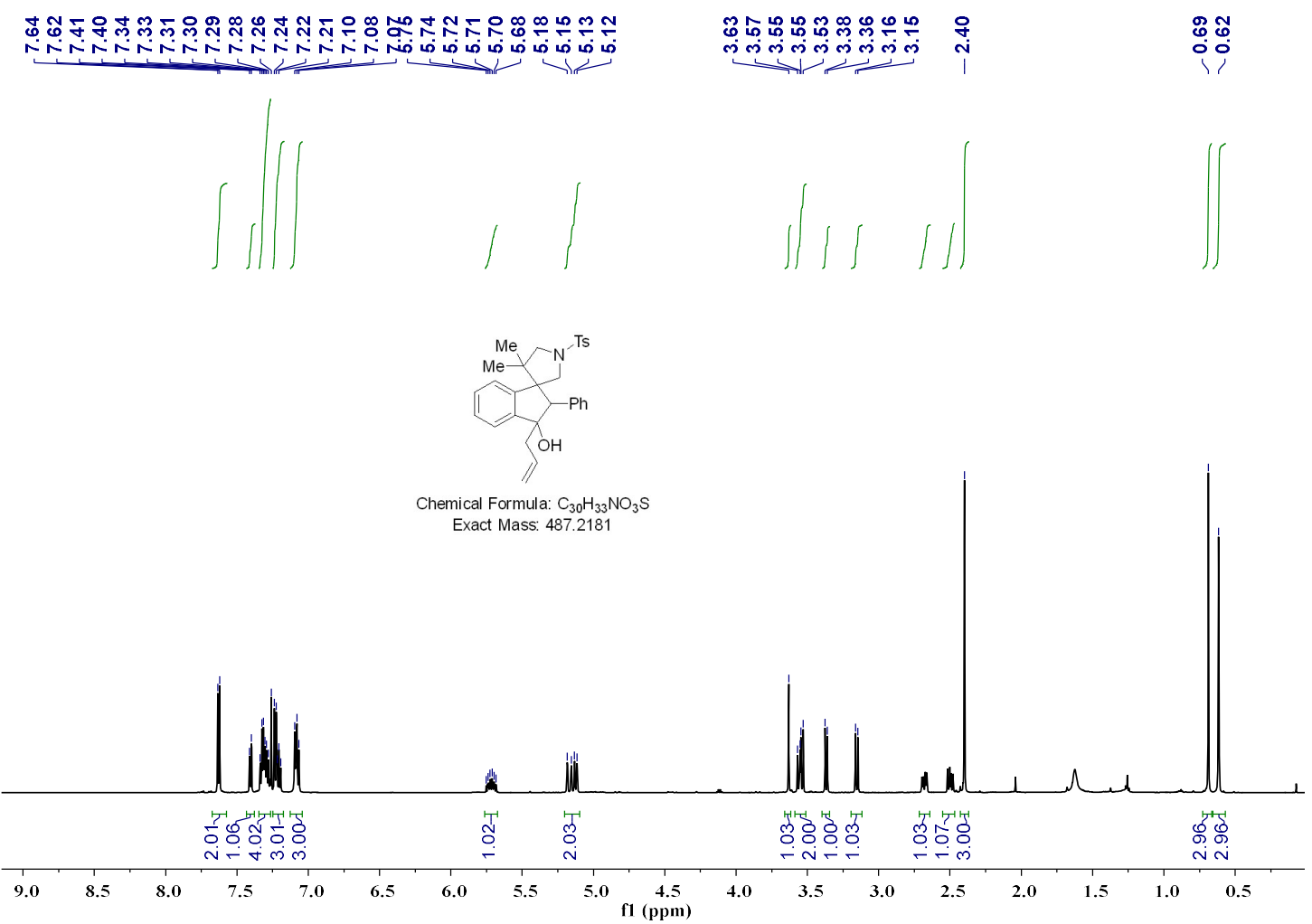

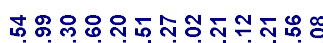

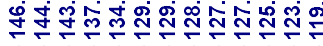

每
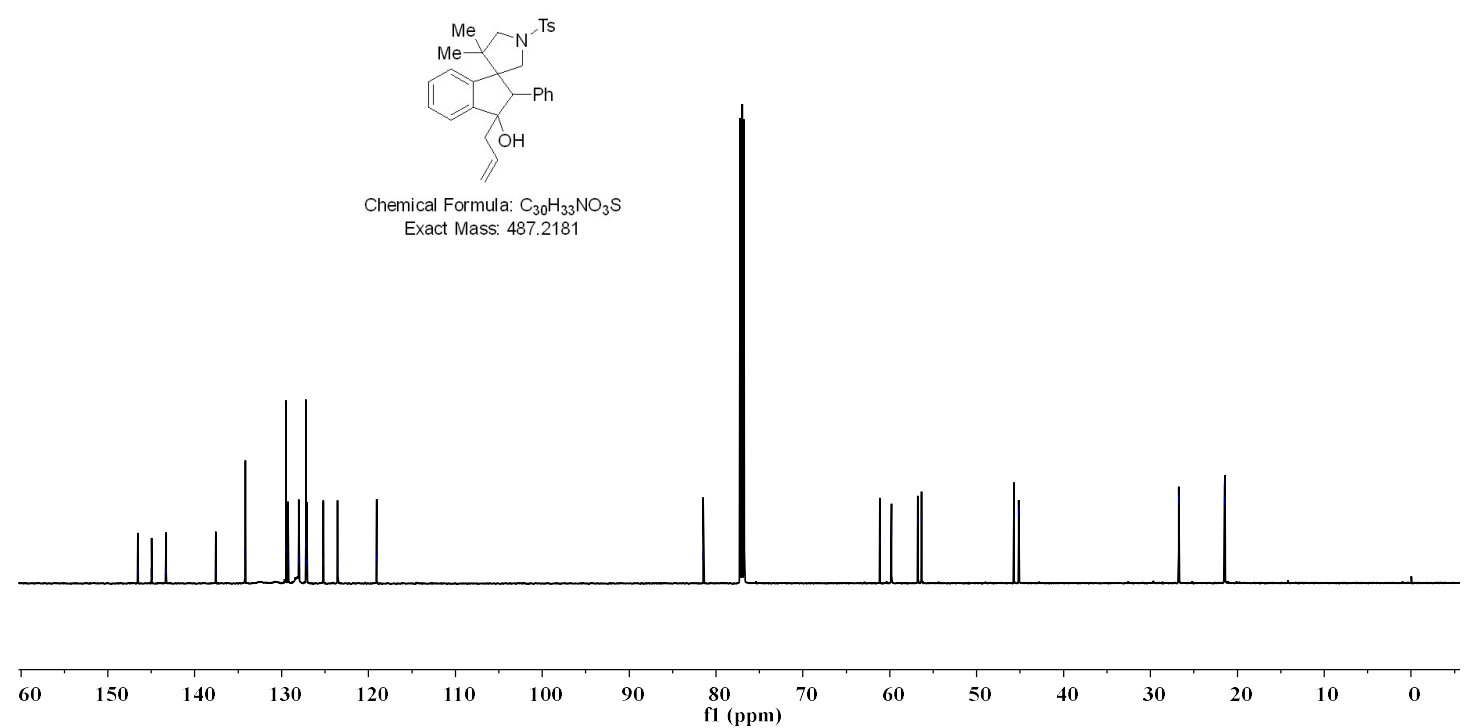

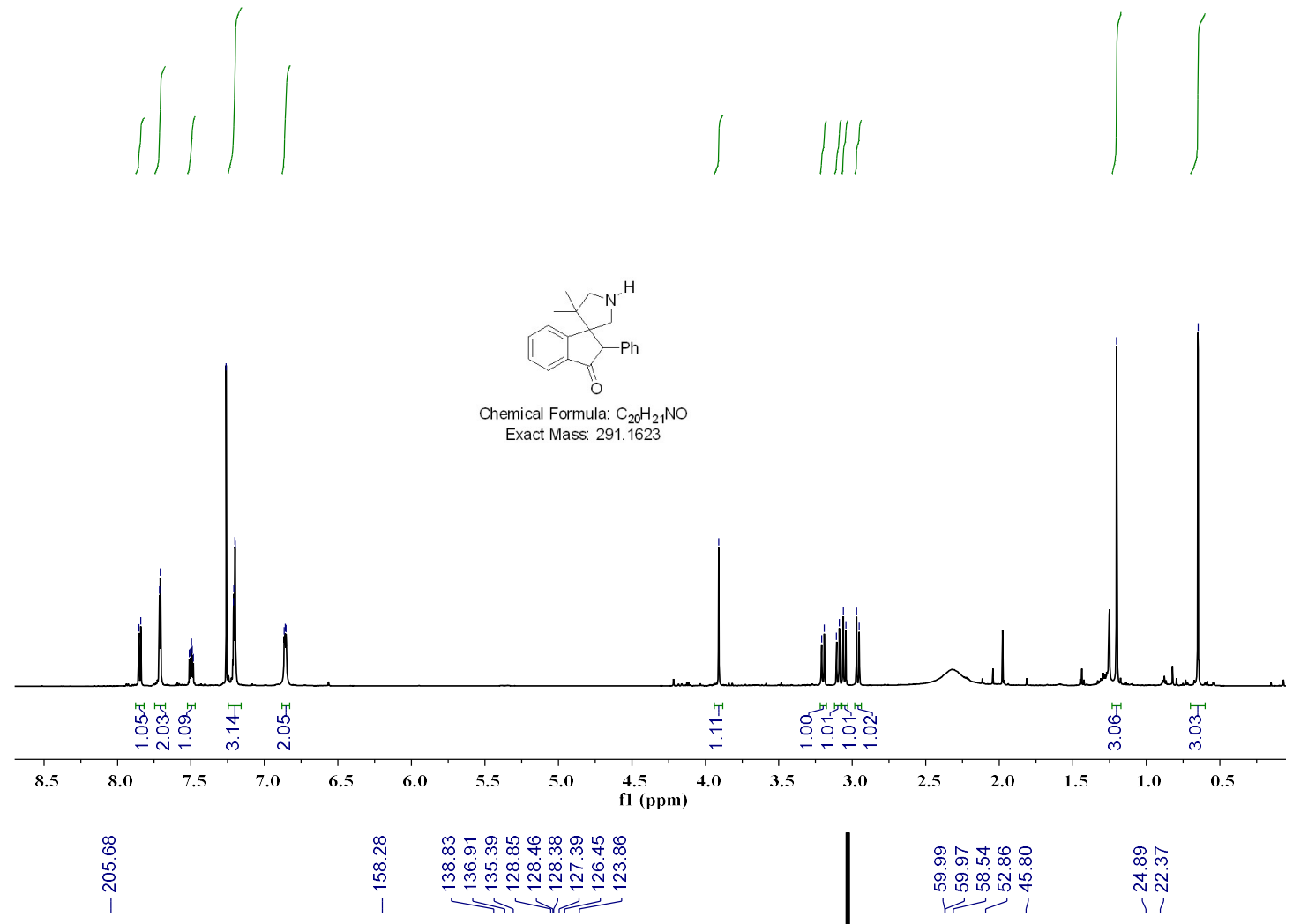

के के

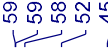

舟色

ป

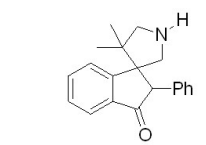

Chemical Formula: $\mathrm{C}_{20} \mathrm{H}_{21} \mathrm{NO}$

Exact Mass: 291.1623

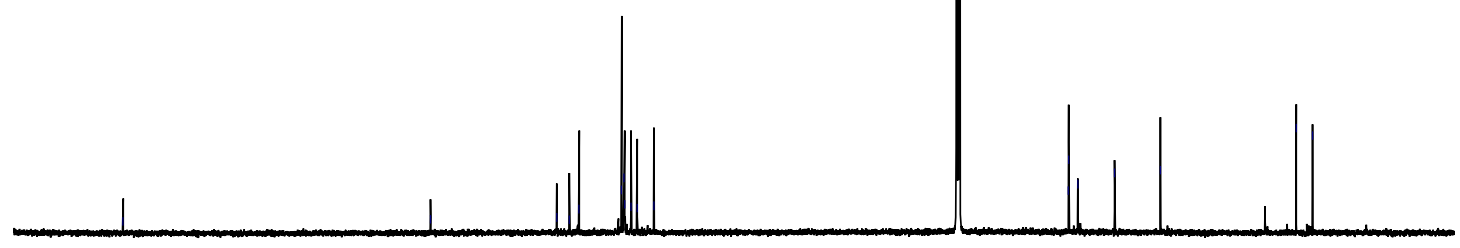

$\begin{array}{llllllllllllllllllllllllllll}220 & 210 & 200 & 190 & 180 & 170 & 160 & 150 & 140 & 130 & 120 & 110 & 100 & 90 & 80 & 70 & 60 & 50 & 40 & 30 & 20 & 10\end{array}$ 

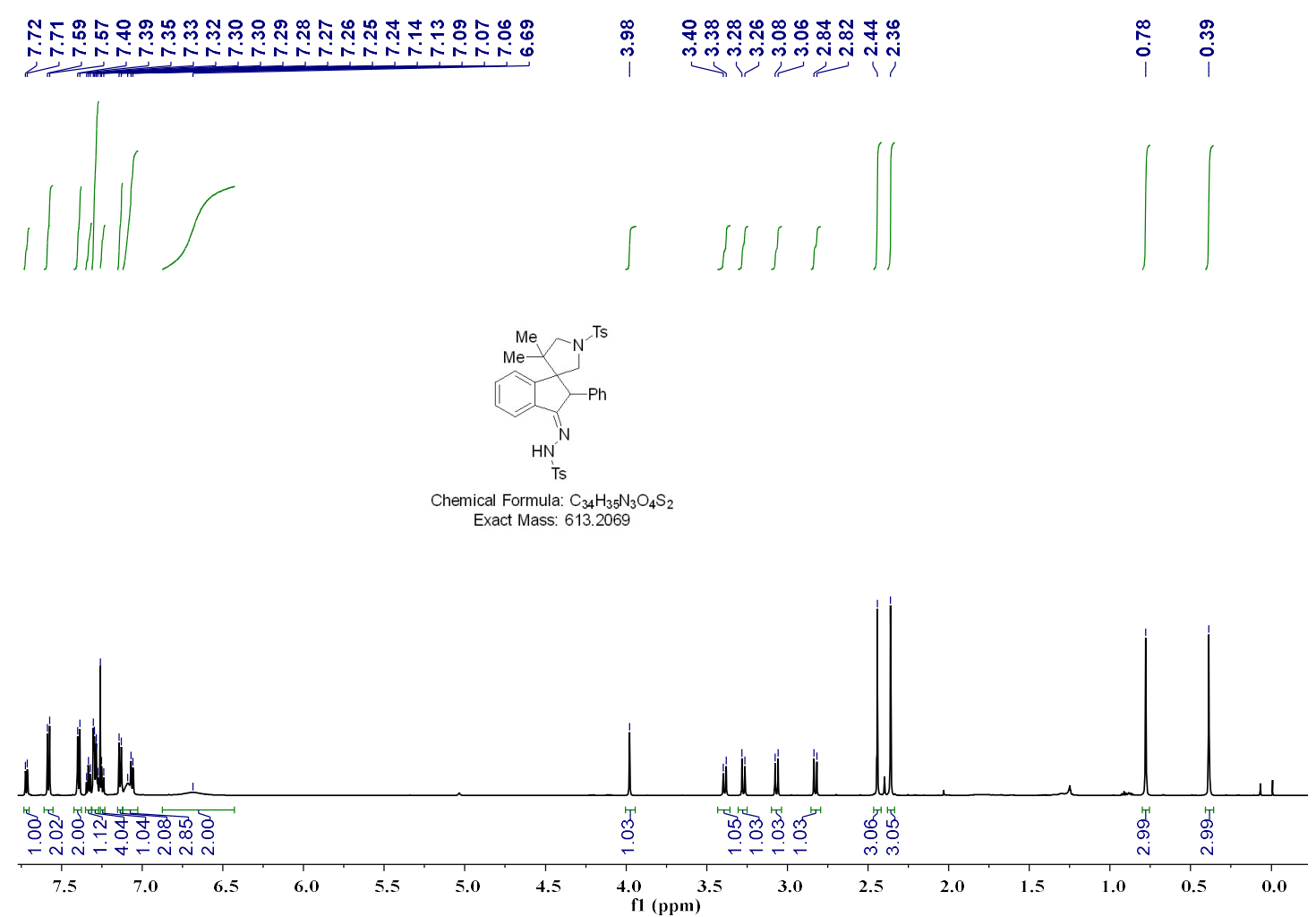
إن

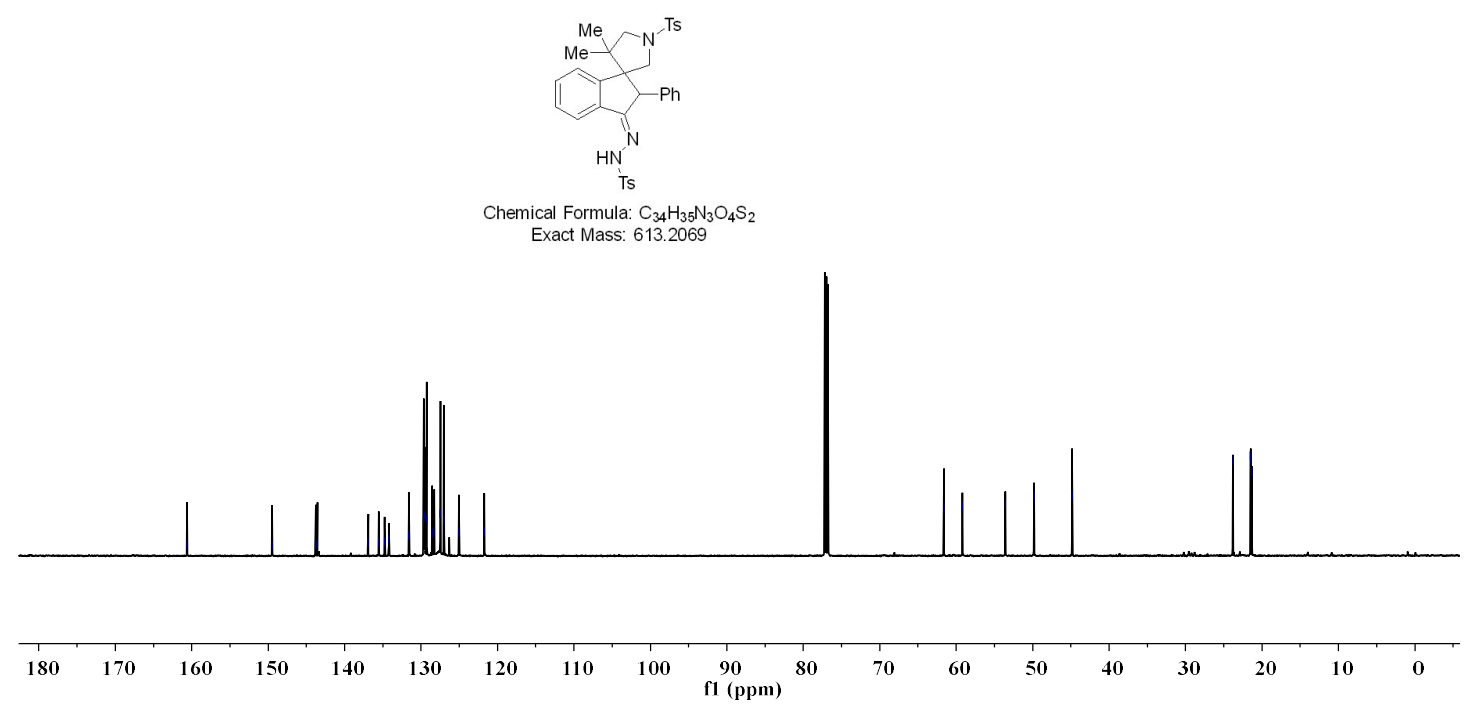


চ

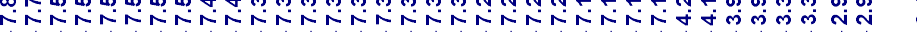

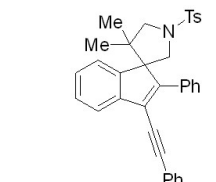

$\mathrm{Ph}$

Chemical Formula: $\mathrm{C}_{35} \mathrm{H}_{31} \mathrm{NO}_{2} \mathrm{~S}$

Exact Mass: 529.2075

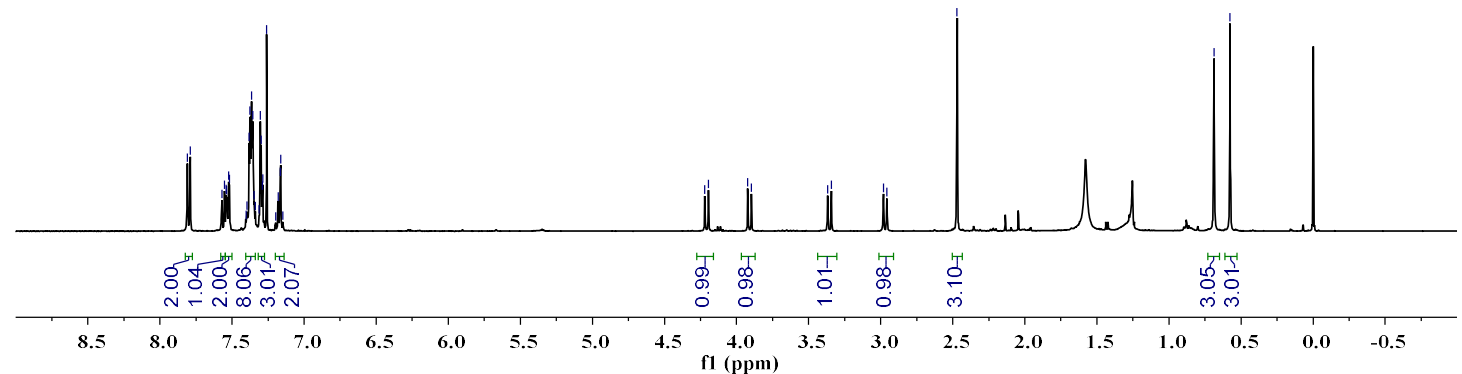

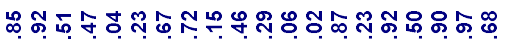

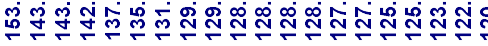

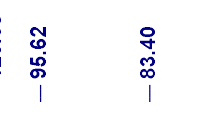

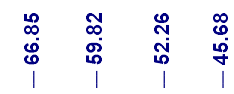

옹요욤

ลั่ กั่

\i,

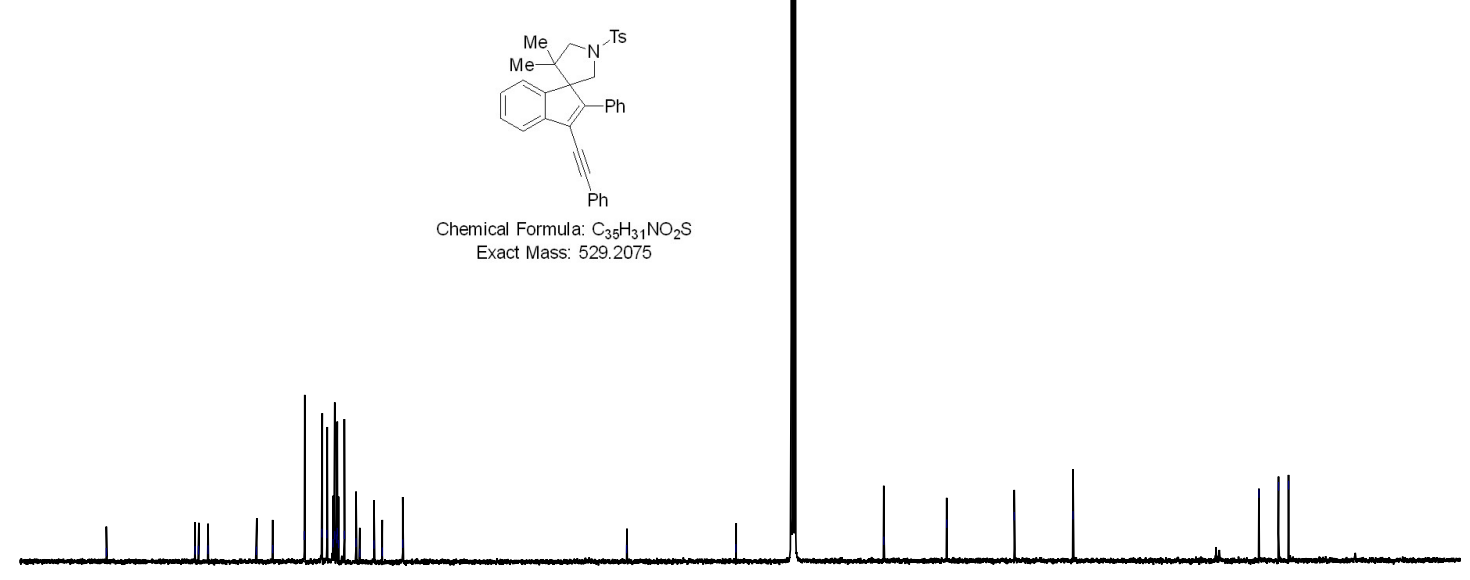

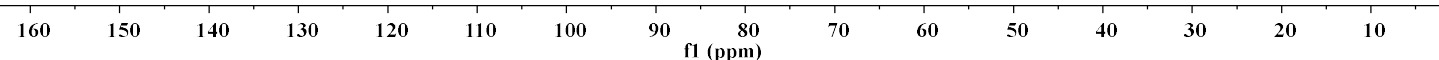




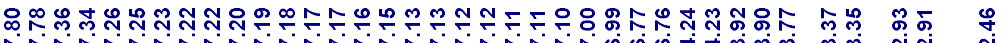

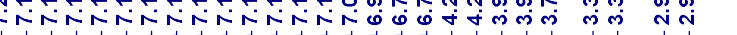
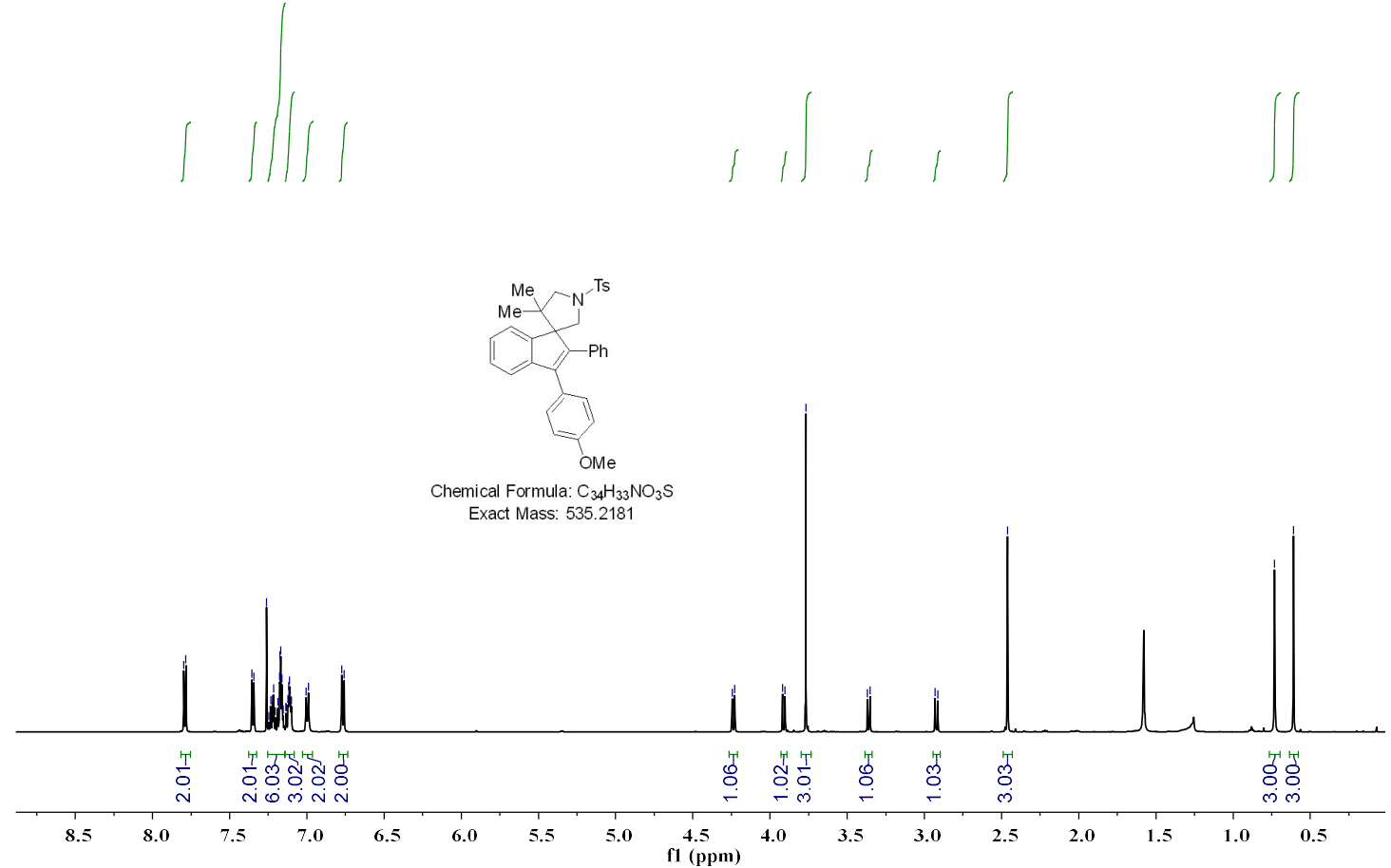

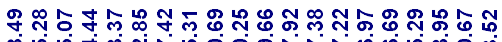

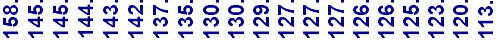

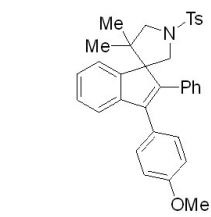

Chemical Formula: $\mathrm{C}_{34} \mathrm{H}_{33} \mathrm{NO}_{3} \mathrm{~S}$ Exact Mass: 535.2181

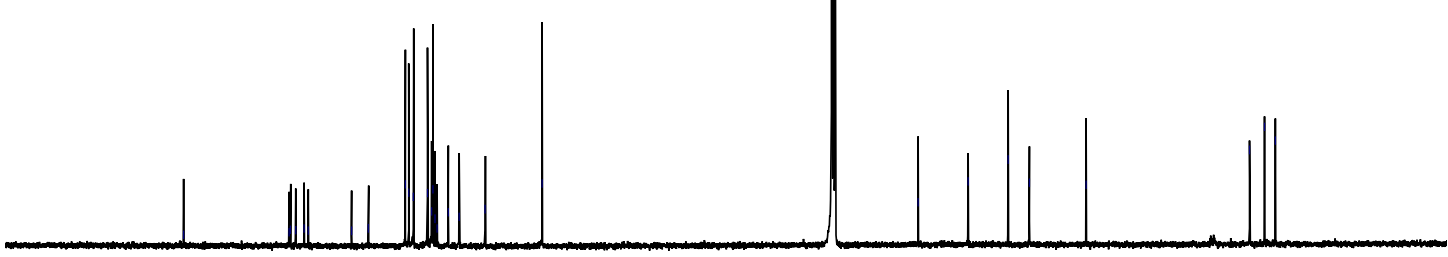

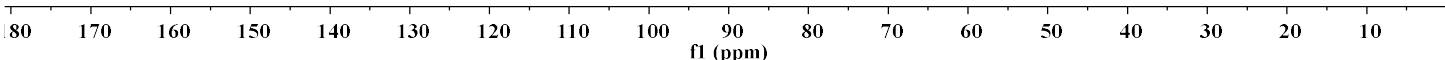




\section{References}

1. (a) Santhoshkumar, R.; Mannathan, S.; Cheng, C.-H. Ligand-Controlled Divergent C-H Functionalization of Aldehydes with Enynes by Cobalt Catalysts. J. Am. Chem. Soc. 2015, 137, 16116-16120. (b) Whyte, A.; Bajohr, J.; Torelli, A.; Lautens, M. Enantioselective CobaltCatalyzed Intermolecular Hydroacylation of 1,6-Enynes. Angew. Chem. Int. Ed. 2020, 59, 16409-16413.. (c) Herath, A.; Li, W.; Montgomery, J. Fully Intermolecular Nickel-Catalyzed Three-Component Couplings via Internal Redox. J. Am. Chem. Soc. 2008, 130, 469-471.

2. Chen, J.; Wang, Y.; Ding, Z.; Kong, W. Synthesis of bridged tricyclo[5.2.1.01,5] decanes via nickel-catalyzed asymmetric domino cyclization of enynones. Nature Commun. 2020, 11, 1882. 3. Snyder, H. R.; Heckert, R. E. A Method for the Rapid Cleavage of Sulfonamides. J. Am. Chem. Soc. 1952, 74, 2006-2009.

4. Zhou, L.; Ye, F.; Ma, J.; Zhang, Y.; Wang, J. Palladium-Catalyzed Oxidative Cross-Coupling of $N$-Tosylhydrazones or Diazoesters with Terminal Alkynes: A Route to Conjugated Enynes. Angew. Chem. Int. Ed. 2011, 50, 3510-3514.

5. Brachet, E.; Hamze, A.; Peyrat, J.; Brion, J.; Alami, M. Pd-Catalyzed Reaction of Sterically Hindered Hydrazones with Aryl Halides: Synthesis of Tetra-Substituted Olefins Related to isoCombretastatin A4. Org. Lett. 2010, 12, 4042-4045. 